\title{
Cartoon-Residual Image Decompositions with Application in Fingerprint Recognition
}

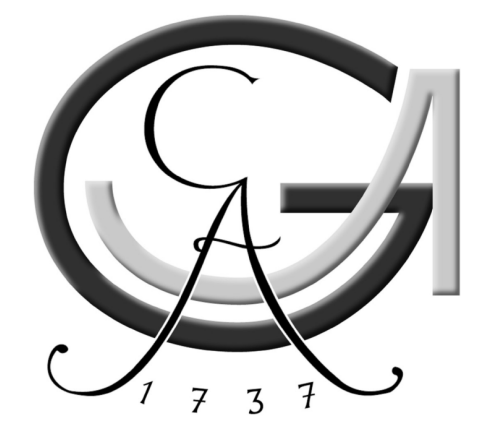

Dissertation

zur Erlangung des mathematisch-naturwissenschaftlichen

Doktorgrades

"Doctor rerum naturalium"

der Georg-August-Universität zu Göttingen

im Promotionsprogramm

"PhD School of Mathematical Sciences (SMS)"

der Georg-August University School of Science (GAUSS)

\author{
vorgelegt von \\ Robin Richter \\ aus Hamburg
}

Göttingen, 2019 


\section{Betreuungsausschuss:}

Prof. Dr. Stephan Huckemann

Felix-Bernstein-Institut für Mathematische Statistik in den Biowissenschaften, Universität Göttingen

Prof. Dr. Gerlind Plonka-Hoch

Institut für Numerische und Angewandte Mathematik, Universität Göttingen

Mitglieder der Prüfungskommission:

Referent:

Prof. Dr. Stephan Huckemann

Felix-Bernstein-Institut für Mathematische Statistik in den Biowissenschaften, Universität Göttingen

Korreferent:

Prof. Dr. Gerlind Plonka-Hoch

Institut für Numerische und Angewandte Mathematik, Universität Göttingen

\section{Weitere Mitglieder der Prüfungskommission:}

Prof. Dr. Dorothea Bahns

Mathematisches Institut, Universität Göttingen

Prof. Dr. Russell Luke

Institut für Numerische und Angewandte Mathematik, Universität Göttingen

Prof. Dr. Anja Sturm

Institut für Mathematische Stochastik, Universität Göttingen

Dr. Frank Werner

Max Planck Institute for Biophysical Chemistry, Göttingen

Institut für Mathematische Stochastik, Universität Göttingen

Tag der mündlichen Prüfung: 06.11.2019 


\section{Preface}

In the last years I had the wonderful opportunity to work at the Institute for Mathematical Stochastics in Göttingen, on the topic of image decompositions and its applications, within the Research Training Group 2088. What made it so wonderful was the great support that I received over this time, from my supervisors, my colleagues and my family and friends. Without all of their help this work would not have been possible.

First and foremost I would like to thank my first supervisor Prof. Dr. Stephan Huckemann for giving me the chance of working on this project and always helping and encouraging me whenever help and encouragement were needed. His dedication to his work and his students was a great source of reassurance, especially in more stressful times. I would like to extend my gratitude also to my second supervisor Prof. Dr. Gerlind Plonka-Hoch, for fruitful discussions and always providing me with a sense of direction throughout my Ph.D.

I would like to thank the committee members Prof. Dr. Dorothea Bahns, Prof. Dr. Russell Luke, Prof. Dr. Anja Sturm and Dr. Frank Werner for taking part in my defence. In particular I would like to thank Prof. Dr. Luke for always having an open door for me, whenever I had a question in continuous optimization, and Dr. Werner for his support in moments I needed guidance and for his insights into inverse problems.

I would to extend my gratitude to Dr. Duy Hoang Thai, without whom this project could not have started. His intuition and first proposals lead to the generalized algorithm analysed in this thesis. I thank him especially for the time and effort he dedicated at the start of my Ph.D to introduce me to the "magic of the frequency domain". Furthermore, I would like to thank Dr. Carsten Gottschlich for his insights into fingerprint analysis.

I will always be incredible grateful for finding a friend in Dr. Benjamin Eltzner, who has been the person I could turn to for any kind of problem from start to finish. It was a joy to work at the IMS and in the RTG, due to the great atmosphere that all colleagues contribute to. I would like to thank all the friends I was able to make over the years, 
Stefan Anell, Christian Böhm, Dr. Miguel Del Alamo, Dr. Anne Hobert, Henning Höllwarth, Dr. Claudia König, Peter Kramlinger, Dr. Anna-Lena Martins, Viacheeslav Natarovskii, Ass. Prof. Dr. Katharina Proksch, Dr. Laura Schneider, Marco Seiler, Dr. Max Sommerfeld, Dr. Kilian Stampfer, Thomas Staudt, Dr. Carla Tameling, Laura Yineth Jula Vanegas, Christoph Weitkamp and Johannes Wieditz.

I would like to thank my family for their love and support. My parents Dr. Silke Richter und Walter Richter, as well as, my siblings Dr. Sonja Longolius and Arne Richter and their respective families, who are the most wonderful people to come home to, no matter where home is.

Finally, I can thank the most important person, my girlfriend Anna Pironi. Being there for me, especially over the last year, meant the world to me. I could not have asked for a more understanding and loving partner in this time. You make my life so much richer.

Financial Support: I would like to thank the RTG 2088 - Discovering Structures in Complex Data of the DFG for their support through project A7.

Previous Publications: Chapter 6 and 7.2 have been previously published in Richter et al. $\left[\mathrm{RGM}^{+} 19\right]$ and are presented here with minor changes. 


\section{Contents}

1 Introduction $\quad 1$

1.1 Cartoon-Residual Decompositions in Applications . . . . . . . . . . 2

1.2 A Remark on the Discrete Setting . . . . . . . . . . . . . . 4

1.3 Locating the Proposed Generalized Algorith in the Literature . . . . . 4

1.3.1 $\ell^{1}$-Regularization with Convolution Operators . . . . . . . . 5

1.3.2 The Cartoon of an Image . . . . . . . . . . . . . . 6

1.4 Literature Review . . . . . . . . . . . . . . . . . . . . . 7

1.4.1 Changing the Data-fidelity Norm . . . . . . . . . . 7

1.4.2 Changing the Regularization-Norm . . . . . . . . . . 8

1.4.3 Changing the Operator . . . . . . . . . . . . 8

1.4.4 Parameter Selection and Extensions Therein . . . . . . . . 9

1.4.5 Convex Optimization . . . . . . . . . . . . . . . . . 9

1.5 The Proposed Generalization . . . . . . . . . . . . . . . . 10

1.5.1 A Feasibility Problem . . . . . . . . . . . . . . . 11

1.5.2 Constrained Double Minimization . . . . . . . . . . . . 12

1.6 Main Results . . . . . . . . . . . . . . . . . . . . . . . . 13

1.6.1 Existence, Convergence and Uniqueness . . . . . . . . . . 13

1.6.2 Experimental Results and Limitations . . . . . . . . . . . 15

1.6.3 Quality Estimation in Fingerprint Analysis . . . . . . . . 16

1.7 Outline of the Thesis . . . . . . . . . . . . . . . . 16

2 Notations and Calculus $\quad 17$

2.1 Linear Algebra . . . . . . . . . . . . . . . . . . . 17

2.1.1 Linear Operators on Matrices . . . . . . . . . . . 18

2.1.2 Matrix Convolutions . . . . . . . . . . . . . . 21

2.1.3 Families of Matrices and Operators thereon . . . . . . . . 23

2.2 Convex Analysis . . . . . . . . . . . . . . . . . . 26

2.3 Mapping Degree Theory . . . . . . . . . . . . . . . 27

2.4 Set-Valued Theory . . . . . . . . . . . . . . . . . . . . . 29 
3 Constrained Double Minimization 31

3.1 Revisiting $\ell^{1}$-Regularizations . . . . . . . . . . . . . 31

3.1.1 Some Equivalent Problem Formulations . . . . . . . . . . . . 31

3.1.2 Loss of Contrast in the $T V-\ell^{2}$-Model . . . . . . . . . . 37

3.1.3 Motivation of the Proposed Generalization . . . . . . . . . 39

3.2 The Generalized Algorithm . . . . . . . . . . . . . . . . . . . . 40

3.2 .1 Input Filters . . . . . . . . . . . . . . . . . . 40

3.2.2 The Generalized Algorithm _. . . . . . . . . . . . 45

3.3 Constrained Double Minimization _ . . . . . . . . . . . . . . 47

4 Convergence Analysis $\quad 51$

4.1 Existence of a Fixed Point $\ldots \ldots \ldots 51$

4.1.1 The Intersection Proposition . . . . . . . . . . . . 53

4.1.2 Preparations and Set-Valued Calculus . . . . . . . . . . 56

4.1.3 Existence of a Fixed Point . . . . . . . . . . . . . . . 63

4.1 .4 Relaxation of the $(\mathrm{CPC}) \ldots \ldots \ldots$

4.2 Convergence to a Fixed Point . . . . . . . . . . . . . . . 70

4.2.1 A Remark on Admissibility . . . . . . . . . . . . . . 75

4.2 .2 Preliminary Lemmas . . . . . . . . . . . . . . . . . 77

4.2.3 Proof of Convergence . . . . . . . . . . . . 80

4.3 Uniqueness of the Fixed Point . . . . . . . . . . . . . . . . . 89

5 Experimental Results $\quad 97$

5.1 Denoising Experiments . . . . . . . . . . . . . . . . . . 97

5.1.1 Compared Models and the PSNR _. . . . . . . . . 98

5.1.2 Images and Covariance Operators . . . . . . . . . . . . . 101

5.1.3 Parameter Optimization and Protocols . . . . . . . . . . . . . 103

5.1.4 Discussion of the Results . . . . . . . . . . . . . . . . 106

5.2 Input Filters Based on Wavelet Frames . . . . . . . . . . . . . 116

5.2 .1 Idea . . . . . . . . . . . . . . . . . . . 116

5.2 .2 Results ..................... . 117

6 Smudge Noise for Fingerprint Quality Estimation and its Validation 123

6.1 Introduction . . . . . . . . . . . . . . . . . . . . . 124

6.1.1 The Quality Feature "Smudge" . . . . . . . . . . . . . . . . 125

6.1.2 The Robust Biometric Quality Validation Scheme (RBQ VS) . 126

6.1.3 Literature on Validation Schemes . . . . . . . . . . . . . 127

6.1 .4 Literature on Fingerprint Quality . . . . . . . . . . . . 128 
6.2 The G3PD Model of Thai and Gottschlich . . . . . . . . . . . . . . 129

6.2.1 Cartoon-Texture-Residual Decomposition . . . . . . . . . . . 129

6.2.2 ROI and Fringe Pattern Extraction . . . . . . . . . . . . . . 129

6.3 The Smudge Noise Quality Estimator (SNoQE) . . . . . . . . . . . 131

6.3.1 Contrast Enhancement . . . . . . . . . . . . . . . . . 131

6.3.2 Smudge Noise Estimation . . . . . . . . . . . . . . . 131

6.3.3 Parameter choices for $r$ and $\alpha \ldots \ldots \ldots \ldots \ldots$

6.4 Robust Biometric Quality Validation Scheme

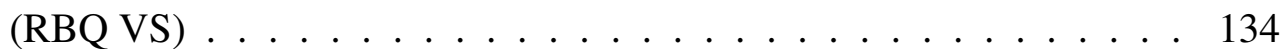

6.5 Comparison Study ～. . . . . . . . . . . . . . . . . 136

6.5 .1 RBQ VS Results . . . . . . . . . . . . . . . . 138

6.5 .2 ERC Curves . . . . . . . . . . . . . . . . 140

6.5.3 Delineation of RBQ VS from ERC Diagnostics . . . . . . . . 143

6.5.4 SNoQE's Strengths and Weaknesses . . . . . . . . . . . . . . 145

$7 \quad$ Discussion and Outlook $\quad 147$

7.1 Cartoon-Residual Decompositions ．. . . . . . . . . . . . . . 147

7.2 Fingerprint Quality Estimation . . . . . . . . . . . . . . . . . 149

$\begin{array}{ll}\text { Appendices } & 151\end{array}$

A Standard Results $\quad 153$

A.1 Linear Algebra . . . . . . . . . . . . . . . . . . 153

A.2 Convex Analysis . . . . . . . . . . . . . . . . . . 159

A.3 Miscellaneous . . . . . . . . . . . . . . . . . . . 166

B Parameters of the Denoising Experiment 169

C Construction of the Wavelet-Frame-Based Filters 173

C.1 Isotropic Polyharmonic B-Spline Wavelet Frames . . . . . . . . . . 173

C.2 Directionality via the Riesz Transform . . . . . . . . . . . . . . 175

C.3 Numerical Adjustments . . . . . . . . . . . . . . . . . . . . 176

$\begin{array}{ll}\text { D Additional Results for SNoQE } & 177\end{array}$

$\begin{array}{ll}\text { Bibliography } & 181\end{array}$ 


\section{List of Symbols}

$\mathbb{N}$

$\mathbb{Z}$

$\mathbb{R}$

$\mathbb{R}_{+}$

$\mathbb{C}$

$\mathbb{R}^{n \times m}$

$\mathbb{C}^{n \times m}$

$\mathbb{R}_{+}^{n \times m}$

$\Gamma_{P}$

$\langle\cdot, \cdot\rangle$

$\|\cdot\|$

$|\cdot|_{1}$

$|\cdot|_{1,1}$

$|\cdot|_{1,2}$

$A^{*}$

$A \odot B$

$\widehat{A}$

F

$\mathfrak{c}_{A}$

$\underline{C}_{B}$

$\underline{\underline{E}} \underline{\underline{C}}$

$\mathrm{S}_{1}$

$\mathrm{S}_{2}$

$\delta_{x y}$

(E)

$\underline{\underline{E}}$

$\overline{\bar{d}}(\cdot, \cdot)$

$\Upsilon: X \rightrightarrows Y$

$\mathcal{N}(0, \mathfrak{S})$ set of positive integers

set of integers

set of real numbers

set of positive real numbers

set of complex numbers

set of real $n$-by- $m$ matrices

set of complex $n$-by- $m$ matrices

set of real $n$-by- $m$ matrices with only positive entries

set of $P$-tuples of real matrices, $\Gamma_{P}=\left(\mathbb{R}^{n \times m}\right)^{P}$

Euclidean scalar product

$\ell^{2}$-norm

$\ell^{1}$-norm on $\mathbb{R}^{n \times m}$

anisotropic $\ell^{1}$-norm on $\Gamma_{P}$

isotropic $\ell^{1}$-norm on $\Gamma_{P}$

adjoint of the operator $A$, w.r.t. $\langle\cdot, \cdot\rangle$

Hadamard product of matrices $A$ and $B$.

Fourier transform of a matrix $A$

discrete Fourier transform

matrix-convolution mapping, from $\mathbb{R}^{n \times m}$ to $\mathbb{R}^{n \times m}$, defined by a convolution matrix $A \in \mathbb{R}^{n \times m}$

matrix-family convolution, mapping from $\mathbb{R}^{n \times m}$ to $\Gamma_{P}$, de-

fined by a matrix-family $\underline{B} \in \Gamma_{P}$

entry-wise matrix-family convolution, mapping from $\Gamma_{P}$ to

$\Gamma_{P}$, defined by a matrix-family $\underline{C} \in \Gamma_{P}$

anisotropic soft-shrinkage function

isotropic soft-shrinkage function

Kronecker delta

identity operator on $\mathbb{R}^{n \times m}$

identity operator on $\Gamma_{P}$

Hausdorff distance

set valued function

multivariate normal distribution with covariance $\mathfrak{S}$ 


\section{CHAPTER 1}

\section{Introduction}

Computing a piecewise smooth approximation of an image, often referred to as a cartoon [MS89], as well as its residual, the texture or noise, proved to be very useful in automatic image processing. Indeed, piecewise smooth approximations are widely used in applications such as segmentation and classification [MMG12, NBCE09], denoising/deblurring [KBPS11, SB07, ROF92, BP10] or shape- and edge-detection [BBD ${ }^{+} 06$, SSD09, XLXJ11, XYXJ12, YGO05]. The notion how a piecewise-smooth approximation should look like, varies from application to application, whether it should remove only noise as in [ROF92, SB07], or, also remove oscillating patterns as in [NBCE09, XLXJ11, XYXJ12], say. Dependent on the application, the composite parts should ideally feature different types of information, for example edges or patterns. Thus, a multitude of cartoon-residual decompositions have been introduced in the literature, often directed at specific applications such as aerial photographs in $\left[\mathrm{BBD}^{+} 06\right]$ or MRI images in [MMG12]. If the types of information to be separated are very specific, for example in finding the fingerprint pattern in a crime scene image, selecting an appropriate decomposition is challenging.

To tackle such problems, this thesis proposes a generalized cartoon-residual decomposition algorithm featuring a high-dimensional set of continuous parameters. It leads to a novel family of constrained double minimization models, that includes a range of known models, e.g. the classical $T V-\ell^{2}$-model of Rudin, Osher and Fatemi [ROF92], as special cases. Moreover, our generalization allows to reformulate the task of choosing a decomposition model as a parameter optimization or training problem. Additionally, for a large family of parameters, the proposed algorithm results, to the best of the author's knowledge, in novel cartoon-residual decompositions, not considered previously.

Our long term goal, that is beyond the scope of this thesis, is to utilize the generality of the proposed algorithm, to tune or train for a most useful cartoon-residual decomposition demanded by a concrete application. Taking a first step, this thesis provides a 
mathematical framework for the proposed algorithm. Moreover, existence, convergence and uniqueness results are shown within this framework for varying subfamilies of parameters, respectively, laying a foundation for possible tuning or training.

Furthermore, as a proof of concept, first experimental results for denoising and textureremoval are presented, that illustrate the potential benefits of the novel decompositions. As an application, this thesis provides a new fingerprint quality estimator based on an existing cartoon-texture-residual decomposition, aiming at measuring large-scale smudge noise.

\subsection{Cartoon-Residual Decompositions in Applications}

Cartoon-residual decompositions are used in many imaging applications, whether on their own, or, as one step in a longer routine. They provide a good tool when processing images by separating large- and small-scale information such as edges and oscillating pattern. The type of information to be extracted might depend on the application at hand, but often one is interested either in (specific) large-scale objects, or in (specific) small-scale patterns.

The most prominent application is image reconstruction of noisy or blurred images. Denoising aims at removing noise from the image, while in deblurring sharp edges should be restored. Cartoon-residual decompositions were used directly for denoising (Rudin et al. [ROF92]), or as a pre-processing step (Sveinsson and Benediktsson [SB07], Selesnick et al. [SGPB14]). They have been considered for deblurring (Chan and Wong [CW98], Choksi et al. [CvGO11]) and artefact removal, for example for clip-art (Wang et al. [WWH06], Xu et al. [XYXJ12]) and astronomical images (Bobichon and Bijaoui [BB97]).

Moreover, cartoon-residual decomposition were used for applications that aimed at, or, profited from removing texture. Image abstraction, as introduced by Winnemöller et al. in [WOG06], was done via cartoon-residual decompositions (Szolgay and Szirány [SS12]), as well as, images composition (Karacan et al. [KEE13], Xu et al. [XYXJ12]) and edge-detection (Xu et al. [XYXJ12]). As a pre-processing step cartoon-residual decompositions was proposed for content-aware image resizing (Karacan et al. [KEE13]). 


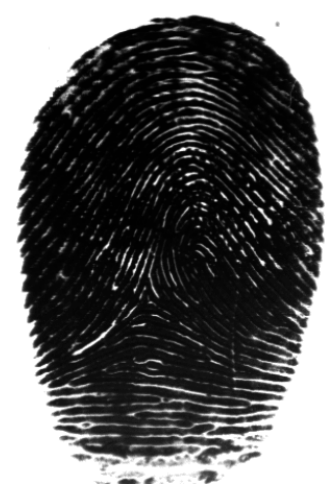

(a)

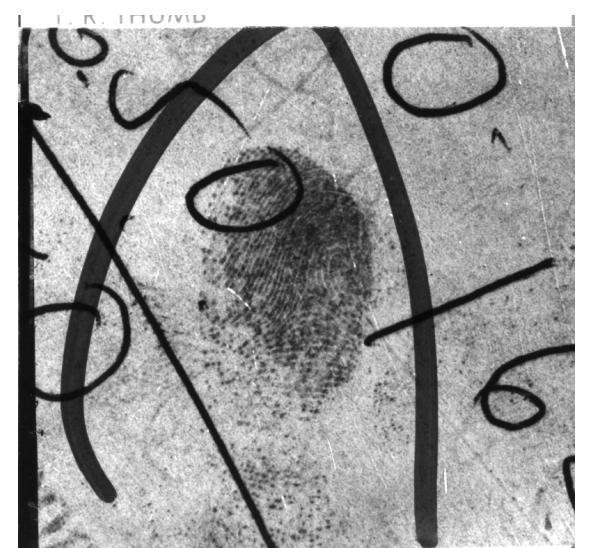

(b)

Figure 1.1: Applications in fingerprint analysis: A fingerprint containing smudge noise (a) and a crime scene fingerprint (b).

In Chapter 6 of this thesis the applicability of cartoon-residual decompositions as a preprocessing step in fingerprint quality estimation is demonstrated. Biometric recognition, in particular based on fingerprints, are used nowadays in a variety of governmental and commercial settings. Most comparison sub-systems rely on position and (possibly) direction of minutiae information. Minutiae are ridge-endings and bifurcations (for an overview over fingerprint analysis see Maltoni et al. [MMJP09]). We show that assessing the presence of smudge noise, see (a) of Figure 1.1 for an example of corruption by smudge, yields a good indicator for fingerprint quality, not represented by the state-of-the-art fingerprint estimators, see Chapter 6.5 of this thesis. To assess this kind of noise we use the cartoon-residual decomposition of Thai and Gottschlich [TG16], that was tailored for its use on fingerprints.

In particular the application of cartoon-texture decompositions in fingerprint analysis, motivates the proposed generalized algorithm of this thesis. Imaging tasks in fingerprint analysis, such as separating automatically the specific fingerprint pattern from other small-scale patterns in crime scene images (see for an example (b) of Figure 1.1), or, detecting automatically specific patterns in fingerprints, such as smudge or scars, are challenging objectives. While considering application driven specialized models is one way to tackle these problems, this thesis aims at making a step towards a large, general model that might be trained or tuned effectively for a large class of applications, not restricted to the fingerprint setting. 


\subsection{A Remark on the Discrete Setting}

Throughout this thesis an image will be considered as a matrix $F \in \mathbb{R}^{n \times m}$. Making this choice, all convergence analysis is set in finite dimensions. The main reason for considering the discrete setting is omitting to consider any discretization effects. Furthermore, in the discrete setting connections between imaging concepts such as regularization, wavelet thresholding and anisotropic diffusion, become more apparent, as discussed in Steidl et al. [SWB $\left.{ }^{+} 04\right]$ and Cai et al. [CDOS12].

Nevertheless, the basis of the presented generalized algorithm is given by models that arose from considering images as continuous functions. To keep the presentation consistent, where needed, discretized versions of the cited continuous models are presented. Lifting the presented generalized algorithm again to a continuous setting might be desirable in future work, opening up the possibility of deeper theoretical understanding of the obtained solutions.

\subsection{Locating the Proposed Generalized Algorith in the Literature}

Our generalized algorithm is rooted in the class of $\ell^{1}$-regularization problems. Many cartoon-residual decompositions have been proposed via the use of a $\ell^{1}$-norm, in particular the well-studied $T V-\ell^{2}$-problem, also called ROF-problem, of Rudin, Osher and Fatemi [ROF92]. In the $T V-\ell^{2}$-problem the total variation norm is used to regularize, which becomes in the discrete setting an $\ell^{1}$-norm of the discrete gradient. Variational problems featuring an $\ell^{1}$-regularizer, in particular in combination with the discrete gradient, are regarded to be well suited for decomposing images into a cartoon and a residual, since they yield a good trade-off between being convex, thus computable via the rich class of convex optimization methods, and, generating a "good" cartoon (Aubert and Kornprobst [AK06]).

Let us give a brief overview over other approaches to imaging applications motivating this thesis. Maybe most related to cartoon-residual decompositions obtained via variational problems are evolutionary diffusion models such as anisotropic diffusion by Perona and Malik [PM90] or its extension by Cattè et al. [CLMC92] (see for an overview the book of Weickert [Wei98]). Note that in the discrete setting Steidl et al. $\left[\mathrm{SWB}^{+} 04\right]$ show that anisotropic diffusion and regularization via $T V-\ell^{2}$ are closely related. 
Especially for denoising images wavelets and generalizations thereof, such as curvelets, have been considered, via statistical testing theory (Donoho and Johnstone [DJ94, DJ95]) and Bayesian estimation (Simoncelli [Sim99], Chang et al. [CYV00a, CYV00b], Portilla et al. [PSWS03]). For separating a cartoon and a texture decomposition wavelet based approaches have been proposed in relation to diffusion (Plonka and Steidl [PS06], Ma and Plonka [MP07]) and in relation to variational problems (Cai et al. [CDOS12]). Moreover, also considering cartoon and texture separation, image filter based schemes have been proposed, based on assessing local extrema (Subr et al. [SSD09]), bilateral filtering (Tomasi and Manduchi [TM98], He et al. [HST13]), (directional) local total variation filters (Buades et al. [BLMV10], Buades and Lisani [BL16b]), or taking non-local means (Buades et al. [BCM05], Pizzaro et al. [PMD $\left.{ }^{+} 10\right]$ ).

Recently there have been approaches to imaging problems via machine learning in particular for denoising (for an overview see McCann et al.[MJU17], Jain and Seung [JS09], Xie et al. [XXC12]). More related to this thesis there have been machine learning approaches for parameter training on existing imaging models, as done in Bayesian wavelet estimation (Zhang et al. [ZZGZ17]), anisotropic diffusion (Chen and Pock [CP17]) and $\ell^{1}$-regularization (Yang et al. [YSLX16]).

\subsection{1 $\ell^{1}$-Regularization with Convolution Operators}

For a general treatment of variational problems in imaging and in particular $\ell^{1}$-regularization see Scherzer et al. [SGG $\left.{ }^{+} 09\right]$. Define first for an input image $F \in \mathbb{R}^{n \times m}$ a general regularization problem by

$$
\begin{aligned}
& \operatorname{minimize} \mathcal{R}(U)+\mu \mathcal{D}(F, U), \\
& \text { over } \quad U \in \mathbb{R}^{n \times m},
\end{aligned}
$$

where $\mathcal{R}: \mathbb{R}^{n \times m} \rightarrow \mathbb{R}$ is called the regularization term, $\mathcal{D}: \mathbb{R}^{n \times m} \times \mathbb{R}^{n \times m} \rightarrow \mathbb{R}$ the datafidelity term and $\mu \in \mathbb{R}_{+}$a balancing parameter. While, the regularization term $\mathcal{R}$ should incorporate a priori knowledge about desired properties of a minimizer of (1.3.1), being piece-wise smooth, say. The data-fidelity term $\mathcal{D}$ should incorporate an appropriate penalization on the solution differing from the original input $F$.

Then, $\ell^{1}$-regularization is given by

$$
\begin{aligned}
& \operatorname{minimize}|Q(U)|_{1, K}+\frac{\mu}{2}\|U-F\|^{2}, \\
& \text { over } \quad U \in \mathbb{R}^{n \times m}
\end{aligned}
$$

where $Q: \mathbb{R}^{n \times m} \rightarrow \mathbb{R}^{q}$ is a linear operator for $q \in \mathbb{N}$ and $|\cdot|_{1, \kappa}$ is an appropriate $\ell^{1}$-norm on $\mathbb{R}^{q}$. 
In this thesis we consider operators $Q: \mathbb{R}^{n \times m} \rightarrow\left(\mathbb{R}^{n \times m}\right)^{P}$ defined via circular convolutions. Circular convolutions cover a range of popular operators used in the literature and have desirable properties such as being diagonalizable by the discrete Fourier transform. Let us denote for $P \in \mathbb{N}$ a family of matrices by $\underline{B}=\left(B_{p}\right)_{p=1}^{P} \in\left(\mathbb{R}^{n \times m}\right)^{P}$ and the space of all families of matrices by

$$
\Gamma_{P}=\left(\mathbb{R}^{n \times m}\right)^{P} .
$$

Then, the matrix-family convolution is defined in the following by

$$
\underline{\mathfrak{C}}_{\underline{B}}(U):=\left(B_{1} * U, B_{2} * U, \ldots, B_{p} * U\right),
$$

where $B * U$ is the usual convolution of matrices (e.g. Mallat [Mal02][p.55]) with components given by

$(B * U)[r, s]=\sum_{k=0}^{n-1} \sum_{\ell=0}^{m-1} B[k, \ell] U[r-k, s-\ell]$, for all $0 \leq k \leq n-1$ and $0 \leq \ell \leq m-1$,

where the index is to be understood modulo $n$ and $m$, respectively. In this setup, the regularization problem becomes

$$
\begin{aligned}
& \operatorname{minimize} \quad\left|\underline{\mathfrak{C}}_{\underline{B}}(U)\right|_{1, \kappa}+\frac{\mu}{2}\|U-F\|^{2}, \\
& \text { over } \quad U \in \mathbb{R}^{n \times m} .
\end{aligned}
$$

A rather prominent example of such a matrix-family convolution is the discrete gradient, given periodic boundary conditions. It can be represented by two matrix convolutions, see Example 2.1.12. Higher order discrete derivative operators can be represented by matrix convolutions in a similar fashion. Moreover, blurring is by definition represented by a matrix convolution. It is also possible to relate wavelet and curvelet frames to matrix-family convolutions. In Steidl et al. $\left[\mathrm{SWB}^{+} 04\right]$ the close relationship of the Haar wavelet and the discrete derivative is shown.

\subsubsection{The Cartoon of an Image}

In their 1989 paper [MS89] Mumford and Shah approximate images via piecewisesmooth functions (for an overview over the Mumford-Shah model and its applications see Aubert and Kornprobst [AK06][Chap.4]). Their underlying image model explicitly ignores among others textures and small-scale patterns and hence, their approximation is called a cartoon of the image. To obtain such an approximation three variants of 
a non-convex functional are given in [MS89], these are, in the continuous setting, well-defined over a space of special functions of bounded variations (De Giorgi and Ambrosio [DGA88], Ambrosio et al. [AMP04]). In order to obtain fast and easily computable solvers similar convex problems have been proposed. This is where the related $T V-\ell^{2}$-problem, mentioned above, comes into play. The $T V-\ell^{2}$-problem is well-defined on the larger space of functions of bounded variations (Meyer [Mey01]) and given, in its unconstrained form (Chambolle and Lions [CL97]), as an $\ell^{1}$-regularization problem by

$$
\begin{aligned}
& \operatorname{minimize}\left|\underline{\mathfrak{C}}_{\underline{D}}(U)\right|_{1,2}+\frac{\mu}{2}\|U-F\|^{2}, \\
& \text { over } \quad U \in \mathbb{R}^{n \times m},
\end{aligned}
$$

where $\underline{\mathfrak{c}}_{\underline{D}}$ is the discrete gradient, cf. Example 2.1.12, and $|\cdot|_{1,2}$ is given by

$$
\left|\left(W_{1}, W_{2}\right)\right|_{1,2}:=\sum_{k=0}^{n-1} \sum_{\ell=0}^{m-1} \sqrt{W_{1}[k, \ell]^{2}+W_{2}[k, \ell]^{2}} .
$$

The $T V-\ell^{2}$-problem, originally proposed for denoising [ROF92], was later also considered for decomposing an image into cartoon and texture components, selecting in (1.3.3) a small regularization parameter $\mu$ (Aujol et al. [AGCO06]). The two wellknown limitations of the $T V-\ell^{2}$-problem are loss-of-contrast, cf. Chapter 3.1.2, and staircasing (Strong and Chan [SC03], Nikolova [Nik00]). Staircasing occurs mostly in denoising problems and manifests itself by approximating linear image patches erroneously as piece-wise constant. Loss-of-contrast on the other hand is an intrinsic features that also poses a challenge in separation into cartoon and texture components, leading to the loss of edges, see Chapter 3.1.2.

\subsection{Literature Review}

Over the decades many extensions and related problems have been proposed for the $T V-\ell^{2}$-problem. In this chapter an overview of the most prominent ones is given, for a review of total variation in imaging see Caselles et al. [CCN15].

\subsubsection{Changing the Data-fidelity Norm}

The $\ell^{2}$-norm in (1.3.3) is a natural first choice as a data-fidelity term, being both easy to handle (smooth, convex) and related to denoising an image corrupted by Gaussian noise (Scherzer et al. [SGG $\left.\left.{ }^{+} 09\right][\S 2.4]\right)$. For the $T V-\ell^{2}$-problem there has been a lot of literature devoted to different data-fidelity norms. 
A large part of models was proposed using the $G$-space (and the related $E$ and $F$ space) introduced by Meyer in [Mey01] as a dual to the subspace of functions of bounded variation, whose derivative belongs to $L^{1}$. The $G$-space is equipped with a $G$-norm that is small if a function is highly oscillating, making it an ideal candidate for a norm of the data-fidelity term in cartoon-residual decompositions. Further analysis on the $G$-space was done by Osher and Scherzer [OS04], Obereder et al. [OOS06] and Haddad and Meyer [HM07]. The $G$-norm or related norms were employed as data-fidelity norm in (1.3.3) by Vese and Osher [VO03, VO04], Osher et al. [OSV03] (generalized by Lieu and Vese [LV08]), Le and Vese [LV05], Aujol et al. [AABFC05], Levine [Lev05], Aujol and Gilboa [AG06] and Garnett et al. [GLMV07, GJLV11]. Notably, some of the proposed data-fidelity norms are related to a change in the regularization parameter as discussed in Chapter 1.4.4, see for example [OSV03, AG06, GLMV07]. Moreover, there has been some work done on decomposing a texture component and a noise component separately in a three-part decomposition for example by Aujol and Chambolle [AC05], Shen [She05] and Tang and He [TH13].

Other replacements of the $\ell^{2}$-norm in the data-fidelity term in (1.3.3) include variants of the $\ell^{1}$-norm to robustify against outliers (Nikolova [Nik04], Chan and Esodoglu [CE05]) and local noise constraints (Bertalmio et al. [BCRS03]). For comparisons of different data-fidelity norms see Nikolova [Nik02], Aujol et al. [AGCO06] and Yin et al. [YGO07].

\subsubsection{Changing the Regularization-Norm}

For the $T V-\ell^{2}$-problem there are two versions of the $\ell^{1}$-regularizer norm, either the isotropic version given in (1.3.4), or an anisotropic version adding the absolute values of all matrix-entries, cf. Chapter 2.1.3. In our proposed algorithm we will include both versions. There have been other extensions proposed, such as replacing the $\ell^{1}$-norm by concave functions (Nikolova [Nik00], Nikolova et al. [NNT10], Xu et al. [XLXJ11]), concatenating the $\ell^{1}$-norm with a smooth function (Le et al. [LLV09]) or proposing a change of both norms in (1.3.3) to an $\ell^{0}-\ell^{1}$-problem (Shen et al. [SCHP12]).

\subsubsection{Changing the Operator}

Changing the discrete derivative in the $T V-\ell^{2}$-problem to a general, linear one we obtain the family of $\ell^{1}$-regularizations given in (1.3.1). In the context of the $T V-\ell^{2}$ problem, higher-order problems were popular in the literature (for an overview see 
Setzer and Steidl [SS08]). In particular against staircasing the inclusion of a higher order derviative (mainly the second) was proposed either replacing or in combination with the original $\underline{\mathfrak{c}}_{\underline{D}}$-regulariation term (Chan et al. [CMM00], Dal Maso et al. [DMFLM09], Bergounioux and Piffet [BP10], Papafitsoros and Schönlieb [PS14]). The generalized $T V$-model by Bredies et al. [BKP10] constitutes a unified approach to higher-order $T V$-problems (extended in Knoll et al. [KBPS11], Bredies [Bre14]).

Then there have been approaches employing a blurring convolution matrix acting on the discrete derivative, in general (Facciolo et al. [FAAC09]) or for a specific non-local means based kernel (Kindermann et al. [KOJ05]). More generally the inclusion of wavelet and curvelet norms in (1.3.3) were proposed (Postelnik et al. [PCP11], Ding and Selesnick [DS15]).

\subsubsection{Parameter Selection and Extensions Therein}

Given a specific task, for example denoising, selecting the right regularization parameter $\mu \in \mathbb{R}_{+}$in (1.3.3) is addressed by Aujol et al. [AGCO06]. Instead of choosing a global parameter $\mu \in \mathbb{R}_{+}$there have been approaches to localize by considering multiple local noise constraints (Bertalmio et al. [BCRS03], Almansa et al. [ACHR06, ABCH08]), generalizing $\mu$ to a function - in the continuous case - hence a matrix $M \in \mathbb{R}^{n \times m}$ acting by component-wise multiplication - in the discrete case - or by choosing $\mu$ as a matrix convolution acting on $F-U$ in the data-fidelity norm itself. The second approach has been done in general (Prasath and Singh [PS10]), or more specifically by selecting $M \in \mathbb{R}^{n \times m}$ via its own minimization problem, constituting a bilevel $T V-\ell^{2}$-problem (Dong et al. [DHRC11], Bredies et al. [BDH13]). Moreover, the latter one - including a matrix convolution in the data-fidelity norm - relates to approximations of the $G$-norm (or related norms) of Meyer [Mey01], recall the references [OSV03, AG06, GLMV07] and see extensions of these models, eg. by Aujol et al. [AGCO06] and Buades et al. [BLMV10, BLMV11].

\subsubsection{Convex Optimization}

A big computational advantage of the $T V-\ell^{2}$-problem, its convexity, the smoothness of its data-fidelity term and the norm of its regularizer being well understood. Hence, there have been multiple algorithms proposed for solving (1.3.3) using the variety of the field of convex optimization. Our generalization is based on the algorithm of $\mathrm{Wu}$ and Tai [WT10] using an augmented Lagrangian approach, more specifically the alternating directions method of multipliers (ADMM). The first introduction of the method 
of multipliers was given in Powell [Pow69] and Hestenes [Hes69]. For a treatment of the extended ADMM in the context of variational inequalities see Gabay [Gab83]. Moreover, for a general result on the convergence of ADMM algorithms see Eckstein and Bertsekas in [EB92][Theorem 8] and references therein, who also specifically show its applicability in the context of augmented Lagrangian approaches.

Before introducing our generalized problem and algorithm, let us give a rough overview over convex optimization algorithms applied for the $T V-\ell^{2}$-problem and its variants, for an in-depth overview we refer the reader to Chambolle and Pock [CP16] and Goldstein et al. [GOSB14].

While the original paper [ROF92] by Rudin, Osher and Fatemi featured a gradient descent algorithm, later a broad range of convex optimization algorithms were specified to solve (1.3.3) or more general minimization problems. Popular approaches include projection algorithms (Chambolle [Cha04], Aujol [Auj09]), the use of Bregman distances (Osher et al. [OBG ${ }^{+}$05], Goldstein and Osher [GO09]), iterative shrinkage-thresholding algorithms (Beck and Teboulle [BT09], Raguet et al. [RFP13]), graph-cut methods (Darbon and Sigelle [DS06a, DS06b]) and forward-backward splitting (Chambolle and Pock [CP11]).

\subsection{The Proposed Generalization}

As discussed in the previous section there have been a multitude of cartoon-residual decompositions introduced in the literature, some more general, some specifically aimed at an application. Considering more unifying models, such as (1.3.2), can help postpone the issue of selecting a model to a later stage, where one might employ training of parameters (as done in Yang et al. [YSLX16]). Moreover, in contrast to directly applying machine learning methods in imaging, the obtained trained parameters allow for more mathematical interpretation of the solutions. Of course, the greater the parameter choices, the potentially better results can be achieved.

In the beginning of this project first numerical tests with an alteration of the ADMM algorithm of Wu and Tai [WT10], different from a minimization problem as in (1.3.1), performed by Duy Hoang Thai, gave promising results, see Figure 1.2. The cartoonresidual decomposition depicted in (b) of Figure 1.2 shows some desirable qualities such as keeping large-scale edges, keeping contrast and removing efficiently texture. On the other hand, new sharp edges can be observed that might be of use in applications, 


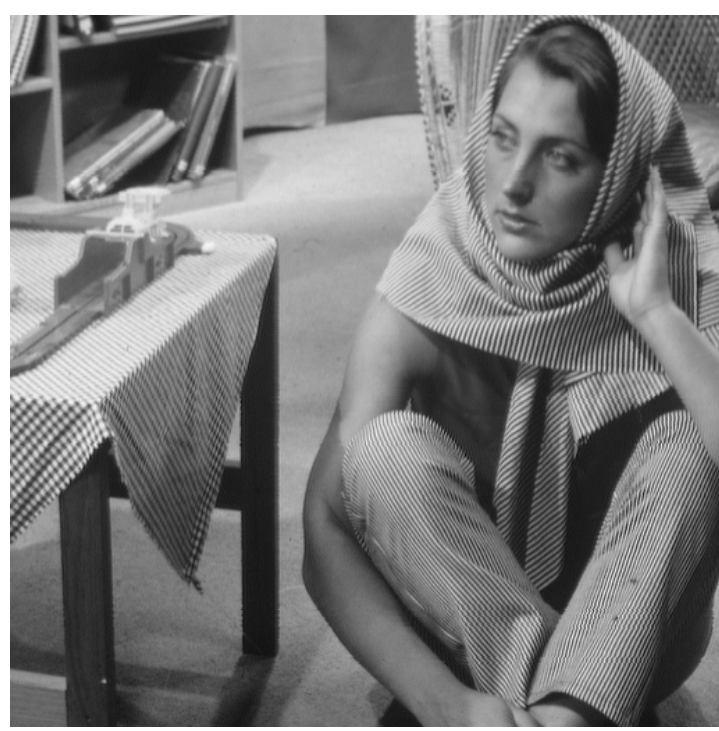

(a)

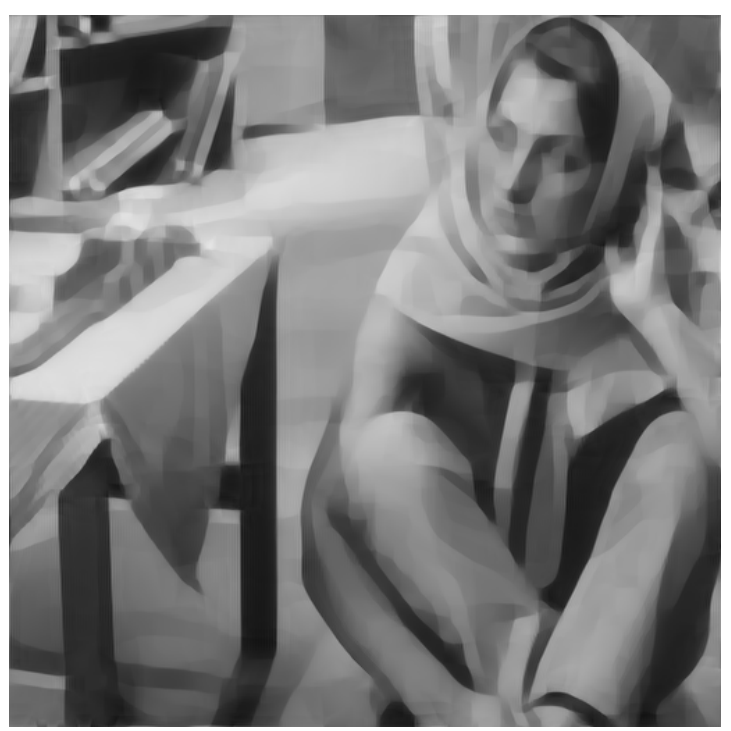

(b)

Figure 1.2: The barbara test image (a), and a cartoon (b) computed by the proposed Algorithm 1.5.2, see Chapter 5.2.

for example the table cloth in (b) features sharp edges where undulated. This thesis provides a generalized algorithm, with (1.3.2) as special cases, of cartoon-residual decompositions, building on these numerical tests.

\subsubsection{A Feasibility Problem}

Pursuing a generalization of the $\ell^{1}$-regularization in (1.3.2) we take a closer look at the specific ADMM algorithm of Wu and Tai [WT10]. It can be reinterpreted as the feasibility problem given below for $\underline{B}=\underline{D}$ and $\kappa=2$, derived in Chapter 3 .

Problem 1.5.1. Given $F \in \mathbb{R}^{n \times m}, \underline{B} \in \Gamma_{P}, \mu \in \mathbb{R}_{+}$and $\kappa \in\{1,2\}$, and let $\beta \in \mathbb{R}_{+}$be arbitrary. Find a point $\left(U^{\dagger}, \underline{W}^{\dagger}, \underline{\lambda}^{\dagger}\right) \in \Gamma_{1+2 P}$ in the intersection of the following three sets

$$
\begin{aligned}
& \Omega_{1}^{\kappa}:=\left\{(U, \underline{W}, \underline{\lambda}) \in \Gamma_{1+2 P}: \underline{W}=\mathrm{S}_{\kappa}\left(\underline{\mathfrak{C}}_{\underline{B}}(U)-\frac{1}{\beta} \underline{\lambda} ; \frac{1}{\beta}\right)\right\}, \\
& \Omega_{2}:=\left\{(U, \underline{W}, \underline{\lambda}) \in \Gamma_{1+2 P}: U=\mu\left(\mu \mathfrak{\mathbb { E }}+\beta \underline{\mathbb{C}}_{\underline{B}}^{*} \underline{\mathfrak{C}}_{\underline{B}}\right)^{-1}(F)\right. \\
& \left.+\beta\left(\mu \mathfrak{\mathbb { E }}+\beta \underline{\mathfrak{C}}_{\underline{B}}^{*} \underline{\mathfrak{C}_{B}}\right)^{-1} \underline{\mathfrak{C}}_{\underline{B}}^{*}\left(\underline{W}+\frac{1}{\beta} \underline{\lambda}\right)\right\}, \\
& \Omega_{C}:=\left\{(U, \underline{W}, \underline{\lambda}) \in \Gamma_{1+2 P}: \underline{\mathfrak{C}}_{\underline{B}}(U)=\underline{W}\right\},
\end{aligned}
$$

where $\mathrm{S}_{\kappa}$ is the isotropic $(\kappa=2)$ or anisotropic $(\kappa=1)$ soft-shrinkage function on $\Gamma_{P}$, see Definition 2.1.17. 


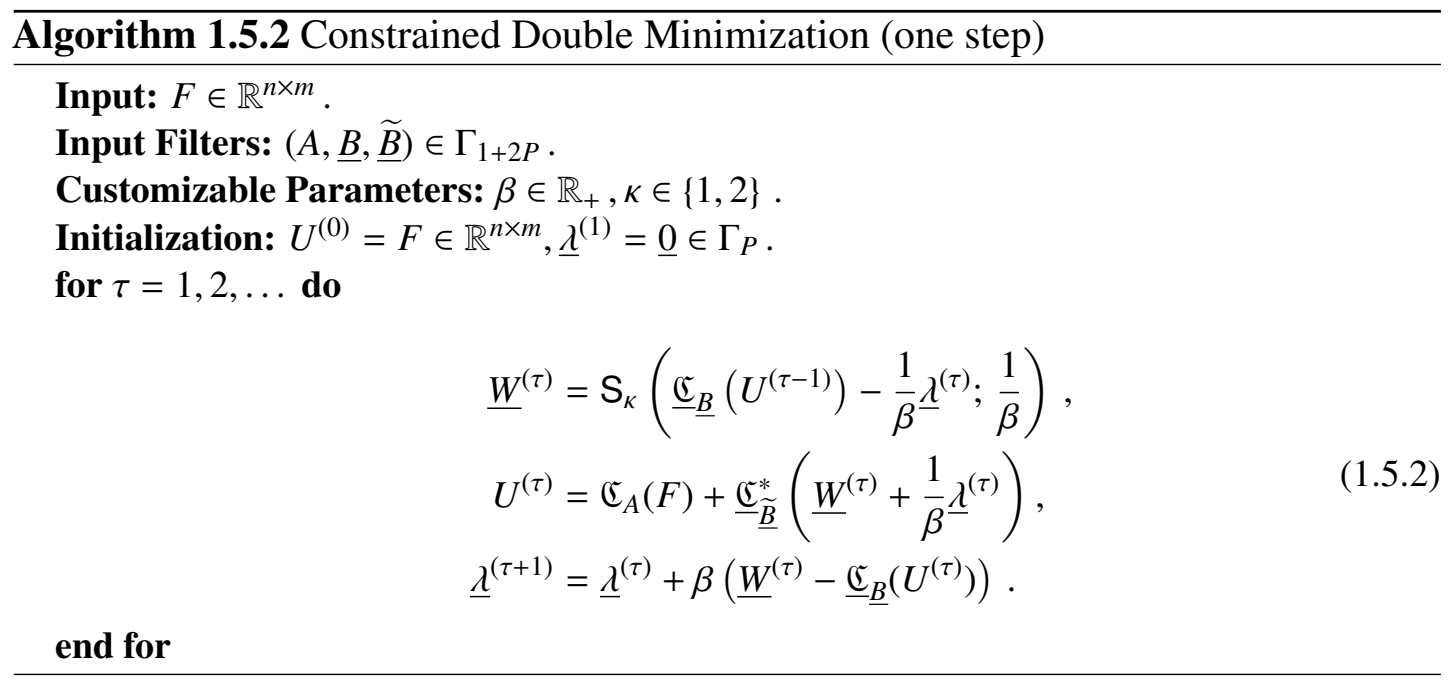

In Chapter 3.2 a generalization of the feasibility Problem 1.5.1, altering the set $\Omega_{2}$, is proposed. To do this let $A \in \mathbb{R}^{n \times m}$ and $\underline{\widetilde{B}} \in \Gamma_{P}$ define two matrix(-family) convolutions $\mathfrak{C}_{A}$ and $\underline{\mathfrak{C}}_{\widetilde{\underline{B}}}$, then we construct

$$
\Omega_{2}^{G}:=\left\{(U, \underline{W}, \underline{\lambda}) \in \Gamma_{1+2 P}: U=\mathfrak{C}_{A}(F)+\underline{\mathfrak{C}}_{\underline{\underline{B}}}^{*}\left(\underline{W}+\frac{1}{\beta} \underline{\lambda}\right)\right\} .
$$

This allows us to state our generalized feasibility Problem 1.5.3, below.

Problem 1.5.3. Given $F \in \mathbb{R}^{n \times m}, \kappa \in\{1,2\}$ and $\beta \in \mathbb{R}_{+}$as well as input filters $(A, \underline{B}, \underline{\widetilde{B}}) \in$ $\Gamma_{1+2 P}$. Find a point $\left(U^{\dagger}, \underline{W^{\dagger}}, \underline{\lambda}^{\dagger}\right) \in \Gamma_{1+2 P}$ in the intersection of the following three sets

$$
\begin{aligned}
& \Omega_{1}^{\kappa}:=\left\{(U, \underline{W}, \underline{\lambda}) \in \Gamma_{1+2 P}: \underline{W}=\mathrm{S}_{\kappa}\left(\underline{\mathfrak{C}}_{\underline{B}}(U)-\frac{1}{\beta} \underline{\lambda} ; \frac{1}{\beta}\right)\right\}, \\
& \Omega_{2}^{G}:=\left\{(U, \underline{W}, \underline{\lambda}) \in \Gamma_{1+2 P}: U=\mathfrak{C}_{A}(F)+\underline{\mathfrak{C}}_{\underline{\underline{B}}}^{*}\left(\underline{W}+\frac{1}{\beta} \underline{\lambda}\right)\right\}, \\
& \Omega_{C}:=\left\{(U, \underline{W}, \underline{\lambda}) \in \Gamma_{1+2 P}: \underline{\mathfrak{C}}_{\underline{B}}(U)=\underline{W}\right\} .
\end{aligned}
$$

As it turns out, the proposed generalization of $\Omega_{2}$ to $\Omega_{2}^{G}$ can be further motivated through an observation regarding the loss-of-contrast in $\ell^{1}$-regularizations, see Section 3.1. Moreover, since Problem 1.5.1 is also the fixed point set of an ADMM algorithm, we can generalize the algorithm in [WT10] in a straight-forward way to obtain the new Algorithm 1.5.2, which features the solutions of Problem 1.5.3 as its fixed point set.

\subsubsection{Constrained Double Minimization}

The main challenge arising by generalizing the feasibility Problem 1.5.1 to Problem 1.5.3 is loosing the feature of a convex $\ell^{1}$-regularization. So as a first step the 
generalized Problem 1.5.3 is shown in Section 3.3 to be equivalent to a variational problem that we will call constrained double minimization. This will be constructed via two functionals $\mathcal{J}_{1}$ and $\mathcal{J}_{2}$ derived from the input $F \in \mathbb{R}^{n \times m}, \kappa \in\{1,2\}, \beta \in \mathbb{R}_{+}$and $(A, \underline{B}, \underline{\widetilde{B}}) \in \Gamma_{1+2 P}$, taking the form, below.

Find $\left(U^{\dagger}, \underline{W}^{\dagger}, \underline{\lambda}^{\dagger}\right) \in \Gamma_{1+2 P}$ such that, $\underline{W}^{\dagger}=\operatorname{argmin}_{\underline{W} \in \Gamma_{P}} \mathcal{J}_{1}\left(U^{\dagger}, \underline{W}, \underline{\lambda}^{\dagger}\right)$, and,$U^{\dagger}=\operatorname{argmin}_{U \in \mathbb{R} n \times m} \mathcal{J}_{2}\left(U, \underline{W}^{\dagger}, \underline{\lambda}^{\dagger}\right)$, under the constraint $\underline{\mathfrak{C}}_{\underline{B}}\left(U^{\dagger}\right)=\underline{W}^{\dagger}$.

\subsection{Main Results}

The main part of this thesis - Chapter 4 - deals with the question of existence of a feasible point in Problem 1.5.3 its uniqueness and the convergence of Algorithm 1.5.2 to such a feasible point. All of the three - existence, uniqueness and convergence are shown to hold under various restrictive conditions on the input matrix-families $(\underline{B}, \underline{\widetilde{B}})$. In Chapter 5 first experiments are conducted analysing benefits of generalizing Problem 1.5.1 to Problem 1.5.3. Last, in applications we show in Chapter 6 how identifying smudge noise can be used in fingerprint quality estimation, using an existing cartoon-texture-residual algorithm.

In the following a detailed overview over the results of this thesis is given.

\subsubsection{Existence, Convergence and Uniqueness}

To characterize the inputs $(\underline{B}, \underline{\widetilde{B}})$ of Algorithm 1.5.2 the following notions are coined, using the notion of the discrete Fourier transform $\widehat{A}$ for a matrix $A$, cf. Definition 2.1.6.

Definition 1.6.1. Let $A \in \mathbb{R}^{n \times m}$ and $\underline{B}=\left(B_{p}\right)_{p=1}^{P} \in \Gamma_{P}$ as well as $\underline{\widetilde{B}}=\left(\widetilde{B}_{p}\right)_{p=1}^{P} \in \Gamma_{P}$, then we call a triple $(A, \underline{B}, \underline{\widetilde{B}})$ input filters for Algorithm 1.5.2. We furthermore introduce the following conditions on $(\underline{B}, \underline{\widetilde{B}})$.

(i) We say that $(\underline{B}, \underline{\widetilde{B}})$ are weakly admissible if for all $1 \leq p \leq P$ and $0 \leq k \leq$ $n-1$ and $0 \leq \ell \leq m-1$ we have

$$
\widehat{\widetilde{B}}_{p}[k, \ell]=\widehat{Y}_{p}[k, \ell] \widehat{B}_{p}[k, \ell]
$$

for some $\underline{Y}=\left(Y_{p}\right)_{p=1}^{P} \in \Gamma_{P}$ with $\widehat{Y}_{p} \in \mathbb{R}_{+}^{n \times m}$, called admissibility matrix-family. 
(ii) We say that $(\underline{B}, \underline{\widetilde{B}})$ are strongly admissible if for all $1 \leq p \leq P$ and $0 \leq k \leq$ $n-1$ and $0 \leq \ell \leq m-1$ we have

$$
\widehat{\widetilde{B}}_{p}[k, \ell]=\widehat{Y}[k, \ell] \widehat{B}_{p}[k, \ell],
$$

for some $Y \in \mathbb{R}^{n \times m}$ with $\widehat{Y} \in \mathbb{R}_{+}^{n \times m}$, called strong admissibility matrix.

(iii) We say that $(\underline{B}, \underline{\widetilde{B}})$ satisfy the non-expansive and positive semidefinite condition (NEPC) if

$$
0 \leq \sum_{p=1}^{P} \overline{\widehat{\widetilde{B}}_{p}[k, \ell]} \widehat{B}_{p}[k, \ell] \leq 1, \quad \text { for all } 0 \leq k \leq n-1 \text { and } 0 \leq \ell \leq m-1 .
$$

(NEPC)

(iv) We say that $(\underline{B}, \underline{\widetilde{B}})$ satisfy the contraction and positive semidefinite condition (CPC) if

$$
0 \leq \sum_{p=1}^{P} \overline{\widetilde{B}}_{p}[k, \ell] \widehat{B}_{p}[k, \ell]<1, \quad \text { for all } 0 \leq k \leq n-1 \text { and } 0 \leq \ell \leq m-1 \text {. (CPC) }
$$

Remark 1.6.2. In Chapter 4.2 .1 it is shown that if $(\underline{B}, \underline{\widetilde{B}})$ satisfies strong admissibility and the (NEPC), Problem 1.5.3 can be rewritten as a minimization problem, minimized by Algorithm 1.5.2. The so obtained minimization problem is related to employing a matrix convolution acting on $F-U$, replacing the balancing parameter $\mu$ in (1.3.2), see Chapter 1.4.4.

For showing existence of a feasible point of Problem 1.5.3, and hence a fixed point of Algorithm 1.5.2, all inputs $(\underline{B}, \underline{\widetilde{B}})$ need to be weakly admissible and satisfy the (CPC).

Theorem 1.6.3. Let $F \in \mathbb{R}^{n \times m}, \kappa \in\{1,2\}, \beta \in \mathbb{R}_{+}$and let $(A, \underline{B}, \underline{\widetilde{B}}) \in \Gamma_{1+2 P}$ be input filters, with $(\underline{B}, \underline{\widetilde{B}})$ weakly admissible and satisfying the (CPC). Given the sets $\Omega_{1}^{\kappa}, \Omega_{2}^{G}$ and $\Omega_{C}$ as defined in Problem (1.5.3), their intersection is non-empty.

Note, that from strong admissibility and the (NEPC), Problem 1.5.3 can be rephrased as a minimization problem. Thus, by Remark 1.6.2 the presented convergence result is not novel, in that it can be derived from classical theory such as [EB92][Theorem 8]. The alternative proof of convergence in Chapter 4.2.3 uses only the variational characterization given by (1.5.4). By doing so the author hopes to shed some light on possible extensions of this result to weak admissibility. In particular, since weak admissibility seems to be desirable as the numerical experiments show improved results, cf. Chapter 5. 
Theorem 1.6.4. Let $F \in \mathbb{R}^{n \times m}, \kappa \in\{1,2\}, \beta \in \mathbb{R}_{+}$and let $(A, \underline{B}, \underline{\widetilde{B}}) \in \Gamma_{1+2 P}$ be input filters, with $(\underline{B}, \underline{\widetilde{B}})$ strongly admissible and satisfying the (NEPC). Assume Algorithm 1.5.2 has at least one fixed point. Then Algorithm 1.5.2 converges to a fixed point for any starting values $U^{(0)} \in \mathbb{R}^{n \times m}$ and $\underline{\lambda}^{(1)} \in \Gamma_{P}$.

Uniqueness can only be guaranteed if $(\underline{B}, \underline{\widetilde{B}})$ are strongly admissible and satisfy the (CPC), for inputs that satisfy only the (NEPC) we obtain a convexity statement. The proofs on uniqueness and convexity of the fixed point set given in this thesis are alternative proofs to classical results, again only using the variational characterization given by (1.5.4).

Theorem 1.6.5. Let $F \in \mathbb{R}^{n \times m}, \kappa \in\{1,2\}, \beta \in \mathbb{R}_{+}$and let $(A, \underline{B}, \underline{\widetilde{B}}) \in \Gamma_{1+2 P}$ be input filters, with $(\underline{B}, \underline{\widetilde{B}})$ strongly admissible and satisfying the $(N E P C)$. Let $\mathcal{X}$ be the set of fixed points of Algorithm 1.5.2. Then, $\mathcal{X}$ is convex.

Theorem 1.6.6. Let $F \in \mathbb{R}^{n \times m}, \kappa \in\{1,2\}, \beta \in \mathbb{R}_{+}$and let $(A, \underline{B}, \underline{\widetilde{B}}) \in \Gamma_{1+2 P}$ be input filters, with $(\underline{B}, \underline{\widetilde{B}})$ strongly admissible and satisfying the $(C P C)$. Then Algorithm 1.5.2 converges to a fixed point with unique $(U, \underline{W})$.

\subsubsection{Experimental Results and Limitations}

The proposed new cartoon-residual decompositions are analysed for potential benefits in denoising and in separating cartoon and texture.

In the case of denoising we consider the model

$$
F=U+\varepsilon
$$

where $\varepsilon$ is some (possibly correlated) multivariate Gaussian noise. Three denoising experiments are conducted in which we compare the classical one-parametric $T V-\ell^{2}$ problem of (1.3.3) with two three-parametric augmented models, within the class of Problem 1.5.3, one of which satisfies strong admissibility, one which only satisfies weak admissibility. We evaluate via the peak signal-to-noise ratio (PSNR) the ability of the new decomposition models to achieve better denoising results than the classical $T V-\ell^{2}$, in general and when trained.

We find that the improvement in PSNR of the augmented models over the classical one is marginal. Notably, however, the improvement seems to manifest itself in keeping better contrast. Results remain stable when trained parameters are applied to unknown noise and to a certain extend also to unknown images. Moreover, the weakly admissible model seems to perform overall better than its strongly admissible counterpart. The experiments underline the need for further analysis whether substantial improvement in denoising is possible. 
For the case of separation of cartoon and texture we consider the model

$$
F=U+V
$$

where $U$ is a cartoon and $V$ a texture component. A wavelet frame based design of $\underline{B}$ and $\underline{\widetilde{B}}$ is proposed based on the work of Van de Ville et al. [VDVBU05]. This novel use of wavelet frames, see Figure 1.2, seems to remove textures, while keeping edges, better than the classical $T V-\ell^{2}$.

\subsubsection{Quality Estimation in Fingerprint Analysis}

We consider the problem of estimating the quality of fingerprints by assessing the presence of smudge noise. This large scale noise is mostly due to too much pressure to the fingerprint scanner. It can obscure, even alter, minutiae pattern in fingerprint images, and thus poses a problem to fingerprint comparison subsystems. Since smudge is related to the absence of oscillating patterns we consider the cartoon-texture-residual decomposition of Thai and Gottschlich [TG16] as an indicator for the presence of smudge. In this application study it is shown that smudge noise relates to the quality of fingerprint images. Moreover, a proposed robust validation scheme and the established error-versus-reject characteristic (ERC) show that the smudge noise quality feature is not sufficiently represented by the state-of-the-art fingerprint quality estimator NFIQ 2.0.

\subsection{Outline of the Thesis}

In Chapter 2 the necessary notations and calculus, used in this thesis, are introduced. In Chapter 3 the original $\ell^{1}$-regularization using convolution operators of (1.3.2) and its relation to the problem of loss-of-contrast are revisited. Chapter 3 goes on to state the generalized Algorithm 1.5.2 and establish equivalent problem formulations for our generalized problem. In Chapter 4 poofs of Theorems 1.6.3, 1.6.4, 1.6.5 and 1.6.6 are given. A numerical proof of concept for the new cartoon-residual decompositions is given in Chapter 5. In Chapter 6 quality estimation in fingerprints is considered. We will conclude the thesis with a discussion of the obtained results and an outlook on future research in Chapter 7. 


\section{CHAPTER 2}

\section{Notations and Calculus}

The following chapter introduces the notation and the calculus in detail which will be used throughout this thesis, in order for this thesis to be self-contained. Since our main objects of interest are discrete images we take here a closer look at spaces of matrices, matrix convolutions as well as families of matrices and operators thereon. The goal is to give an exhaustive overview over the (basic) linear algebra that will be utilized in later statements. The introduced notation is, to the best of the author's knowledge, novel and can be found in more generality in the book of Bourbaki [Bou89][\$10]. Moreover, in the proofs of Chapter 3 and 4 we will need results in convex analysis, set-valued calculus and mapping degree theory. We will state the definitions and results needed, following the books of Ekeland and Tèmam [ET99] in the case of convex analysis, Aubin and Ekeland [AE84] in the case of set-valued function theory and Outerelo and Ruiz [OR09] in the case of mapping-degree theory.

\subsection{Linear Algebra}

Let us first motivate the later introduced matrix notation. As it is often the case we will decode a two-dimensional, discrete signal (image) as a matrix $F \in \mathbb{R}^{n \times m}$. When describing operators on $\mathbb{R}^{n \times m}$ vectorization of the matrices involved is often performed in order to represent the operators as matrices, as for example in [WYYZ08]. We would like to avoid these kind of background operations. So, in the following we will not loose the two-directional structure of our objects and instead work with the more structured object of linear operators on the space $\mathbb{C}^{n \times m}$, fully defined via

$\mathfrak{M}:=\left(M_{r, s}\right)_{r=0, s=0}^{n-1, m-1}$, where $M_{r, s}=\left(M_{r, s}[k, \ell]\right)_{k=0, \ell=0}^{n-1, m-1}, \quad 0 \leq r \leq n-1,0 \leq s \leq m-1$, 
and acting on a matrix $A \in \mathbb{C}^{n \times m}$ via

$$
\mathfrak{M}(A):=\left(\operatorname{tr}\left(M_{r, s} A^{T}\right)\right)_{r=0, s=0}^{n-1, m-1}=\left(\sum_{k=0}^{n-1} \sum_{\ell=0}^{m-1} M_{r, s}[k, \ell] A[k, \ell]\right)_{r=0, s=0}^{n-1, m-1} .
$$

We stay in this chapter in $\mathbb{C}^{n \times m}$, even though our images lie in $\mathbb{R}^{n \times m}$, in order to fully utilize the discrete Fourier transform and its diagonalizing properties for matrix convolutions. The above representation follows the general definition of matrices in Bourbaki [Bou89][II,§10,Def.1,Def.3]. As one would expect all results for complexvalued matrices also hold for matrix-valued matrices $\mathfrak{M}$. In this chapter we will state the basic results about general matrices, using the introduced notation that is tailored to our needs. Additionally, we extend these notions to families of matrices and operators thereon, this will be particularly useful when defining multi-matrix valued operators such as the discrete gradient operator on $\mathbb{R}^{n \times m}$.

\subsubsection{Linear Operators on Matrices}

Let us start with some conventions, that will come in particularly handy when dealing with the discrete Fourier transform.

Convention 2.1.1. For any matrix $A \in \mathbb{C}^{n \times m}$ we will denote its indices by $A[k, \ell]$ for $0 \leq k \leq n-1$ and $0 \leq \ell \leq m-1$. Most importantly, matrix entries are indexed in $\mathbb{Z} / n \mathbb{Z} \times$ $\mathbb{Z} / m \mathbb{Z}$ and any matrix entry exceeding the range of $\{0,1, \ldots n-1\} \times\{0,1, \ldots, m-1\}$ should be considered as its modulo correspondence within this range (see Bredies et al. [BKP10][§4.1]).

Furthermore for a matrix $A \in \mathbb{C}^{n \times m}$, denote by $A^{*}$ its adjoint, i.e. its transposed and complex conjugate.

We introduce the usual inner product on $\mathbb{C}^{n \times m}$ by

$$
\langle A, B\rangle=\sum_{k=0}^{n-1} \sum_{\ell=0}^{m-1} A[k, \ell] \overline{B[k, \ell]}=\operatorname{tr}\left(A B^{*}\right),
$$

yielding the Frobeniusnorm

$$
\|A\|_{2}=\sqrt{\sum_{k=0}^{n-1} \sum_{\ell=0}^{m-1} A[k, \ell] \overline{A[k, \ell]}}=\sqrt{\operatorname{tr}\left(A A^{*}\right)} .
$$

Furthermore, we will denote by $\odot$ the Hadamard (component-wise) product of $A, B \in$ 
$\mathbb{C}^{n \times m}$, where $A \odot B$ is defined by

$(A \odot B)[k, \ell]=A[k, \ell] B[k, \ell]$, for all $0 \leq k \leq n-1$ and $0 \leq \ell \leq m-1$.

As already mentioned, we define a linear operator $\mathfrak{M}$ on $\mathbb{C}^{n \times m}$ via (2.1.1) and its action via (2.1.2). Note that every linear operator $\mathbb{C}^{n \times m} \rightarrow \mathbb{C}^{n \times m}$ is of that form. Let us collect some straight-forward correspondences to usual matrix calculus in the following definition.

Definition 2.1.2 ([Bou89](II,§10)). (i) We define the identity linear operator on $\mathbb{C}^{n \times m}$ as $\mathfrak{E}=\left(E_{r, s}\right)_{r=0, s=0}^{n-1, m-1}$ via matrix-entries

$$
E_{r, s}[k, \ell]=\delta_{r k} \delta_{s \ell},
$$

where $\delta_{x y}$ is the Kronecker delta.

(ii) We define the adjoint of a linear operator $\mathfrak{M}$ on $\mathbb{C}^{n \times m}$ to be an operator $\mathfrak{M}^{*}$ such that for all $A, B \in \mathbb{C}^{n \times m}$ we have

$$
\langle\mathfrak{M}(A), B\rangle=\left\langle A, \mathfrak{M}^{*}(B)\right\rangle .
$$

See (i) of Lemma 2.1.3 for a characterization of the adjoint.

(iii) We say a linear operator $\mathfrak{M}=\left(M_{r, s}\right)_{r=0, s=0}^{n-1, m-1}$ on $\mathbb{C}^{n \times m}$ is unitary if we have

$$
\mathfrak{M} \circ \mathfrak{M}^{*}=\mathfrak{E} .
$$

(iv) We say a linear operator $\mathfrak{M}=\left(M_{r, s}\right)_{r=0, s=0}^{n-1, m-1}$ on $\mathbb{C}^{n \times m}$ is diagonal if we have

$$
M_{r, s}[k, \ell]=0 \text { for all }(k, \ell) \neq(r, s),
$$

or equivalently,

$$
\mathfrak{M}=\left(\alpha_{r, s} E_{r, s}\right)_{r=0, s=0}^{n-1, m-1},
$$

for suitable $\alpha_{r, s} \in \mathbb{C}$.

(v) We say a linear operator $\mathfrak{M}=\left(M_{r, s}\right)_{r=0, s=0}^{n-1, m-1}$ on $\mathbb{C}^{n \times m}$ is diagonalized by a linear operator $\mathfrak{N}$ on $\mathbb{C}^{n \times m}$, if $\mathfrak{N}$ is invertible and $\mathfrak{N} \circ \mathfrak{M}_{\circ} \mathfrak{N}^{-1}$ is a diagonal linear operator on $\mathbb{C}^{n \times m}$.

Let us first gather some properties of such operators, for the proofs see Appendix A.1. 
Lemma 2.1.3 ([Bou89](II,§10)). (i) Let $\mathfrak{M}$ be a linear operator on $\mathbb{C}^{n \times m}$, if its adjoint is given by $\mathfrak{M}^{*}=\left(N_{r, s}\right)_{r=0, s=0}^{n-1, m-1}$, we have

$$
N_{r, s}[k, \ell]=\overline{M_{k, \ell}[r, s]} .
$$

(ii) For any linear operator $\mathfrak{M}=\left(M_{r, s}\right)_{r=0, s=0}^{n-1, m-1}$ and $\mathfrak{N}=\left(N_{r, s}\right)_{r=0, s=0}^{n-1, m-1}$ on $\mathbb{C}^{n \times m}$ the operator $\mathfrak{M} \circ \mathfrak{N}$ is again a linear operator on $\mathbb{C}^{n \times m}$ with matrix entries $\left(L_{x, y}\right)_{x=0, y=0}^{n-1, m-1}$ given by

$L_{x, y}[k, \ell]=\sum_{r=0}^{n-1} \sum_{s=0}^{m-1} M_{x, y}[r, s] N_{r, s}[k, \ell]$ for all $0 \leq x, k \leq n-1$ and $0 \leq y, \ell \leq m-1$.

(iii) For any linear operators $\mathfrak{M}=\left(M_{r, s}\right)_{r=0, s=0}^{n-1, m-1}$ and $\mathfrak{N}=\left(N_{r, s}\right)_{r=0, s=0}^{n-1, m-1}$ on $\mathbb{C}^{n \times m}$ we have

$$
\left(\mathfrak{M}{ }^{*} \mathfrak{N}\right)^{*}=\mathfrak{H}^{*} \mathfrak{M}
$$

(iv) Given a unitary linear operator $\mathfrak{N}=\left(N_{r, s}\right)_{r=0, s=0}^{n-1, m-1}$ on $\mathbb{C}^{n \times m}$, then we have

$$
\left\langle N_{r, s}, N_{k, \ell}\right\rangle=\delta_{r k} \delta_{s \ell},
$$

for all $0 \leq k, r \leq n-1$ and all $0 \leq \ell, s \leq m-1$. Furthermore,

$$
\mathfrak{N}\left(\overline{N_{r, s}}\right)=E_{r, s} \text { for all } 0 \leq r \leq n-1 \text { and } 0 \leq s \leq m-1
$$

(v) Given a linear operator $\mathfrak{M}$ on $\mathbb{C}^{n \times m}$ that is diagonalized by an unitary linear operator $\mathfrak{A}$ on $\mathbb{C}^{n \times m}$, let $\left(P_{r, s}\right)_{r=0, s=0}^{n-1, m-1}$ be defined by

$$
\left(P_{r, s}\right)_{r=0, s=0}^{n-1, m-1}:=\mathfrak{N} \mathfrak{M} \mathfrak{N}^{*},
$$

then

$$
P_{r, s}[r, s] \text { for } 0 \leq r \leq n-1 \text { and } 0 \leq s \leq m-1 \text {, }
$$

constitute all eigenvalues of $\mathfrak{M}$ to the eigenmatrices

$$
\overline{N_{r, s}} \text { for } 0 \leq r \leq n-1 \text { and } 0 \leq s \leq m-1 \text {, }
$$

respectively.

In (ii) of the above Lemma 2.1.3 the product of linear operators on $\mathbb{C}^{n \times m}$ is shown to be the analogue to the product of two matrices. For this reason we will from now on 
always write simply $\mathfrak{M P}$ instead of $\mathfrak{M} \circ \mathfrak{N}$, having always this product in mind.

\subsubsection{Matrix Convolutions}

Now we are ready to define matrix convolutions, special linear operators on $\mathbb{C}^{n \times m}$, that will be used repeatedly.

Definition 2.1.4 ([Mal98](p.55)). Let $A \in \mathbb{C}^{n \times m}$ be a matrix, then the linear operator $\mathfrak{C}_{A}: \mathbb{C}^{n \times m} \rightarrow \mathbb{C}^{n \times m}$ defined via

$$
\mathfrak{C}_{A}=\left(A_{r, s}\right)_{r=0, s=0}^{n-1, m-1},
$$

where

$$
A_{r, s}[k, \ell]:=A[r-k, s-\ell] \text { for } 0 \leq k, r \leq n-1 \text { and } 0 \leq \ell, s \leq m-1 \text {, }
$$

is called a discrete circular convolution (called circular convolution in [Ma198, p.55]). If $A \in \mathbb{R}^{n \times m}$ we will simply denote it in the following as a matrix convolution.

We call the matrix $A$ the convolution matrix or simply the filter.

Remark 2.1.5. Let $A \in \mathbb{R}^{n \times m}$ be a filter.

(i) For any matrix $B \in \mathbb{R}^{n \times m}$ we have $\mathfrak{C}_{A}(B) \in \mathbb{R}^{n \times m}$.

(ii) The adjoint of the matrix convolution $\mathfrak{C}_{A}$ with filter $A$ is again a matrix convolution defined via the filter À given by

$$
\grave{A}[k, \ell]:=\overline{A[-k,-\ell]} \text {, for all } 0 \leq k \leq n-1 \text { and } 0 \leq \ell \leq m-1 \text {. }
$$

We will denote the adjoint of $\mathfrak{C}_{A}$ by $\mathfrak{C}_{A}^{*}$.

Part (i) follows directly from the definition of a matrix convolution, while part (ii) follows directly from (i) of Lemma 2.1.3.

One of the most important properties of a matrix convolution is that it is diagonalizable over $\mathbb{C}^{n \times m}$ via the discrete Fourier transform.

Definition 2.1.6 ([Mal98](p.56)). We define the discrete Fourier transform (DFT) of a matrix $A \in \mathbb{C}^{n \times m}$ as the matrix $\widehat{A} \in \mathbb{C}^{n \times m}$ defined by

$$
\widehat{A}[k, \ell]=\sum_{r=0}^{n-1} \sum_{s=0}^{m-1} A[r, s] \omega_{n}^{k r} \omega_{m}^{\ell s},
$$

for $0 \leq k \leq n-1$ and $0 \leq \ell \leq m-1$, where $\omega_{t}=e^{-\frac{2 \pi i}{t}}$ for $t \in \mathbb{N}$. 
Let us make some more remarks about the DFT before we draw the connection to matrix convolutions.

Remark 2.1.7. (i) The DFT is a linear operator on $\mathbb{C}^{n \times m}$ given by $\mathfrak{F}=\left(F_{r, s}\right)_{r=0, s=0}^{n-1, m-1}$ with

$$
F_{r, s}[k, \ell]=\omega_{n}^{k r} \omega_{m}^{\ell s}=e^{-\frac{2 \pi i k r}{n}-\frac{2 \pi i \ell s}{m}},
$$

for $0 \leq k \leq n-1$ and $0 \leq \ell \leq m-1$ giving

$$
\widehat{A}=\mathfrak{F}(A) \quad \forall A \in \mathbb{C}^{n \times m}
$$

(ii) Note that $\mathfrak{F}$ is invertible, its inverse is given by the linear operator $\mathfrak{F}^{-1}=$ $\left(\widetilde{F}_{r, s}\right)_{r=0, s=0}^{n-1, m-1}$ with entries

$$
\widetilde{F}_{r, s}[k, \ell]=\frac{\overline{F_{r, s}[k, \ell]}}{n m}=\frac{\omega_{n}^{-k r} \omega_{m}^{-\ell s}}{n m}
$$

Finally, let us summarize some well-known results brought into our setting of linear operators on $\mathbb{C}^{n \times m}$. For the proofs we refer to Appendix A.1.

Lemma 2.1.8 ([SS03](Chap.7)). (i) The linear operator $\frac{1}{\sqrt{n m}} \mathfrak{F}$ is a unitary operator. In particular,

$$
\mathfrak{F}\left(\bar{F}_{r, s}\right)=\sqrt{n m} E_{r, s},
$$

for all $0 \leq r \leq n-1$ and $0 \leq s \leq m-1$.

(ii) Let $\mathfrak{V}_{A}$ be a matrix convolution and $\mathfrak{V}_{A}^{*}$ its adjoint with filters $A$ and $B$, respectively. Then

$$
\widehat{B}[k, \ell]=\overline{\widehat{A}[k, \ell]} .
$$

(iii) Let $\mathfrak{C}_{A}$ be a matrix convolution with filter $A$, then we have for any matrix $B \in \mathbb{C}^{n \times m}$

$$
\widehat{C_{A}(B)}=\widehat{A} \odot \widehat{B}
$$

(iv) Applying the discrete Fourier transform we diagonalize a matrix convolution. Additionally, any two matrix convolutions $\mathfrak{C}_{A}, \mathfrak{C}_{B}: \mathbb{C}^{n \times m} \rightarrow \mathbb{C}^{n \times m}$ commute, i.e.

$$
\mathfrak{C}_{A} \circ \mathfrak{C}_{B}(L)=\mathfrak{C}_{B} \circ \mathfrak{C}_{A}(L),
$$

for all matrices $L \in \mathbb{C}^{n \times m}$. 
Lemma 2.1.9 (Plancherel). Let $A, B \in \mathbb{C}^{n \times m}$, then we have

$$
\langle A, B\rangle=n m\langle\widehat{A}, \widehat{B}\rangle \text {. }
$$

\subsubsection{Families of Matrices and Operators thereon}

In this chapter we generalize these concepts even further by formalizing multi-matrixvalued operators such as the discrete gradient.

Convention 2.1.10. For $P \in \mathbb{N}_{\geq 2}$ we define the space

$$
\Gamma_{P}:=\left\{\underline{W}:=\left(W_{1}, W_{2}, \ldots, W_{P}\right): W_{p} \in \mathbb{R}^{n \times m} \text { for all } 1 \leq p \leq P\right\}
$$

we will denote a family of matrices by underlining, i.e. $\underline{W} \in \Gamma_{P}$.

On the space $\Gamma_{P}$ we define the usual Euclidean inner product for all $\underline{W}, \underline{V} \in \Gamma_{P}$ via

$$
\langle\underline{W}, \underline{V}\rangle_{\Gamma_{P}}=\sum_{p=1}^{P}\left\langle W_{p}, V_{p}\right\rangle
$$

yielding the usual $\ell^{2}$-norm

$$
\|\underline{W}\|_{2}:=\sqrt{\sum_{p=1}^{P} \sum_{k=0}^{n-1} \sum_{\ell=0}^{m-1}\left|W_{p}[k, \ell]\right|^{2}},
$$

Furthermore, we can define an isotropic $\ell^{1}$-norm by

$$
|\underline{W}|_{1,2}:=\sum_{k=0}^{n-1} \sum_{\ell=0}^{m-1}\left\|\left(W_{1}[k, \ell], \ldots, W_{P}[k, \ell]\right)^{T}\right\|,
$$

we will call this isotropic, since it relates to the isotropic Sobolev-semi-norm, see [SGG ${ }^{+}$09][p.258259], for $p=2$. Its anisotropic counterpart, $p=1 \mathrm{in}$ [SGG ${ }^{+} 09$ ], is defined in the discrete setting as the anisotropic $\ell^{1}$-norm via

$$
|\underline{W}|_{1,1}:=\sum_{p=1}^{P} \sum_{k=0}^{n-1} \sum_{\ell=0}^{m-1}\left|W_{p}[k, \ell]\right| .
$$

See also Cai et al.'s definition of $\|\cdot\|_{1, p}$ in [CDOS12]

After we have specified the space of $P$-sized families of matrices we now consider two types of operators: One mapping from $\mathbb{R}^{n \times m}$ to $\Gamma_{P}$ and one that maps from $\Gamma_{P}$ to itself. We will not characterize all linear operators here, as done in the previous chapter for the 
space of matrices, but will restrict ourselves to the type of matrix convolution operators that will be needed later.

Definition 2.1.11. (i) A linear operator $\underline{\mathfrak{C}}_{B}: \mathbb{R}^{n \times m} \rightarrow \Gamma_{P}$ defined via a family of real-valued matrices $\underline{B} \in \Gamma_{P}$ acting on a matrix $A \in \mathbb{R}^{n \times m}$ by

$$
\underline{\mathfrak{C}}_{\underline{B}}(A)=\left(\mathfrak{C}_{B_{1}}(A), \ldots, \mathfrak{C}_{B_{P}}(A)\right) \in \Gamma_{P},
$$

is called a matrix-family convolution.

(ii) Furthermore, a linear operator $\underline{\underline{C}}_{C}: \Gamma_{P} \rightarrow \Gamma_{P}$ that is defined via a family of realvalued matrices $\underline{C} \in \Gamma_{P}$ but acting on a matrix-family $\underline{W} \in \Gamma_{P}$ via component-wise matrix convolutions, i.e.

$$
\underline{\underline{\mathfrak{c}}}_{\underline{C}}(\underline{W})=\left(\mathfrak{c}_{C_{1}}\left(W_{1}\right), \ldots \mathfrak{C}_{C_{P}}\left(W_{P}\right)\right),
$$

is called an entry-wise matrix-family convolution. In order to distinguish these operators from matrix-family convolutions we introduce here the convention of double-underlining these operators.

Let us give a prominent example of a matrix-family convolution.

Example 2.1.12. For $P=2$, the discrete gradient defined on $\mathbb{R}^{n \times m}$ with periodic boundary conditions (see for example [WT10]) can be written as a matrix-family convolution by

$$
\underline{\mathfrak{C}}_{\underline{D}}: \mathbb{R}^{n \times m} \rightarrow \Gamma_{2}, \quad U \mapsto\left(\mathfrak{C}_{D_{1}}(U), \mathfrak{C}_{D_{2}}(U)\right)^{T},
$$

where

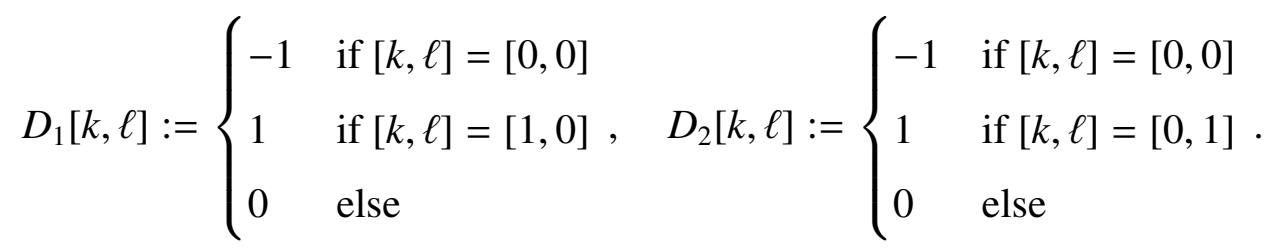

In the last part of this chapter let us collect some linear algebra results for such operators that will come in handy. For proofs we refer again to Appendix A.1.

Lemma 2.1.13. (i) Let $\underline{\mathfrak{C}}_{\underline{B}}$ be a matrix-family convolution defined as above then its 
adjoint is given by

$$
\left(\underline{\mathfrak{C}}_{\underline{B}}\right)^{*}: \Gamma_{P} \rightarrow \mathbb{R}^{n \times m} ; \quad \underline{W} \mapsto \sum_{p=1}^{P} \mathfrak{C}_{B_{p}}^{*}\left(W_{p}\right)
$$

(ii) Let $\underline{\underline{\underline{C}}}_{\underline{\underline{C}}}$ be an entry-wise matrix-family convolution defined as above then its adjoint is given by

$$
\left(\underline{\underline{\mathfrak{C}}}_{\underline{C}}\right)^{*}: \Gamma_{P} \rightarrow \Gamma_{P} ; \quad \underline{W} \mapsto\left(\mathfrak{C}_{C_{1}}^{*}\left(W_{1}\right), \ldots \mathfrak{C}_{C_{P}}^{*}\left(W_{P}\right)\right),
$$

Convention 2.1.14. We simply write $\mathfrak{C}_{B_{p}}^{*}, \underline{\mathfrak{C}}_{\underline{B}}^{*}$ and $\underline{\mathfrak{C}}_{C}^{*}$ to denote the adjoint instead of writing $\left(\mathfrak{C}_{B_{p}}\right)^{*},\left(\underline{\mathfrak{C}}_{\underline{B}}\right)^{*}$ and $\left(\underline{\mathfrak{C}}_{\underline{C}}\right)^{*}$, respectively.

Example 2.1.15 (2.1.12-continued). For the discrete derivative $\underline{\mathfrak{c}}_{\underline{D}}$ its adjoint is given by

$$
\underline{\mathfrak{C}}_{\underline{D}}^{*}(\underline{W})=\sum_{p=1}^{2} \mathfrak{C}_{D_{p}}^{*}\left(W_{p}\right)
$$

where $\mathfrak{C}_{D_{p}}^{*}$ is defined via filters $\widetilde{D}_{p}$ given by

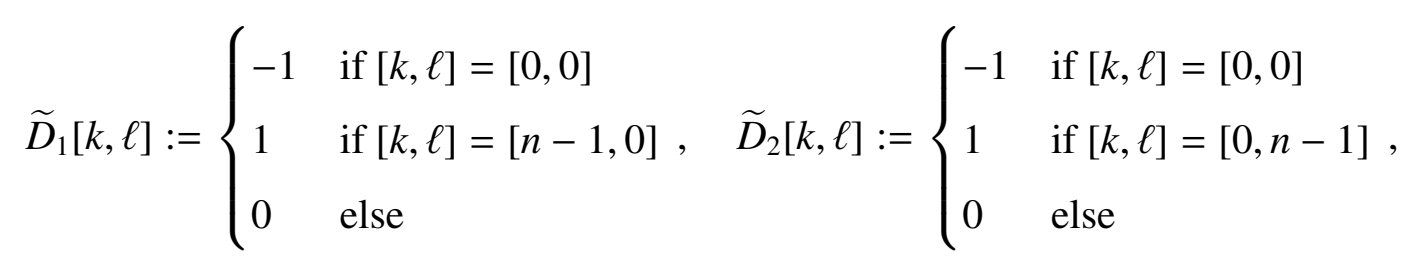

according to (i) of Lemma 2.1.3 and Lemma 2.1.13. Which coincides with the discrete divergence for periodic boundary conditions (e.g. [WT10]).

Now we consider a commutation result for the interplay of entry-wise matrix-family convolutions and matrix-family convolutions.

Lemma 2.1.16. Let $\underline{\underline{\mathbb{C}}}_{\underline{C}}: \Gamma_{P} \rightarrow \Gamma_{P}$ be an entry-wise matrix-family convolution where $C_{p}=C$ for all $1 \leq p \leq P$ and let $\underline{\mathfrak{E}}_{\underline{B}}: \mathbb{C}^{n \times m} \rightarrow \Gamma_{P}$ be a matrix-family convolution as defined before. Then we have

$$
\underline{\mathfrak{C}}_{\underline{B}}^{*} \underline{\underline{C}}_{\underline{C}}(\underline{W})=\mathfrak{C}_{C} \underline{\mathfrak{C}}_{\underline{B}}^{*}(\underline{W}) .
$$

Proof.

$$
\begin{aligned}
\underline{\mathfrak{C}}_{\underline{B}}^{*} \underline{\underline{\mathfrak{C}}} \underline{\underline{C}}(\underline{W}) & =\sum_{p=1}^{P} \mathfrak{C}_{B_{p}}^{*}\left(\mathfrak{C}_{C}\left(W_{p}\right)\right)=\sum_{p=1}^{P} \mathfrak{C}_{C}\left(\mathfrak{C}_{B_{p}}^{*}\left(W_{p}\right)\right) \\
& =\mathfrak{C}_{C}\left(\sum_{p=1}^{P} \mathfrak{C}_{B_{p}}^{*}\left(W_{p}\right)\right)=\mathfrak{C}_{C} \underline{\mathfrak{C}_{\underline{B}}^{*}}(\underline{W}) .
\end{aligned}
$$


The second equality is given by (iv) of Lemma 2.1.8 and the third one by linearity of matrix convolutions.

Finally in this chapter, let us define the soft-shrinkage functions on $\Gamma_{P}$.

Definition 2.1.17. (i) The anisotropic soft-shrinkage function $S_{1}: \Gamma_{P} \times \mathbb{R}_{+} \rightarrow \Gamma_{P}$ for $P \in \mathbb{N}$ is defined in the following as

$$
\begin{aligned}
& \mathrm{S}_{1}(\underline{W} ; \beta):=\left(\left(\mathrm{s}_{1}\left(W_{p}[k, \ell] ; \beta\right)\right)_{k=0, \ell=0}^{n-1, m-1}\right)_{1 \leq p \leq P}, \\
& \text { with } \quad \mathrm{s}_{1}(x ; \beta):= \begin{cases}x-\beta & \text { if } x>\beta \\
0 & \text { if } x \in[-\beta, \beta] . \\
x+\beta & \text { if } x<-\beta\end{cases}
\end{aligned}
$$

(ii) The isotropic soft-shrinkage function $\mathrm{S}_{2}: \Gamma_{P} \times \mathbb{R}_{+} \rightarrow \Gamma_{P}$ for $P \in \mathbb{N}$ is defined in the following as

$$
\left.\mathrm{S}_{2}(\underline{W} ; \beta):=\left(\left(\mathrm{S}_{2}\left(\left(\left(W_{p}[k, \ell]\right)_{p=1}^{P}\right)\right)^{T} ; \beta\right)[p]\right)_{k=0, \ell=0}^{n-1, m-1}\right)_{1 \leq p \leq P}
$$

where for $x=\left(x_{1}, x_{2}, \ldots, x_{P}\right)^{T}$ we have

$$
\mathrm{s}_{2}(x ; \beta):=\frac{x}{\|x\|} \max (0,\|x\|-\beta) .
$$

\subsection{Convex Analysis}

We state in the following the definition of the subdifferential and an important variational inequality tailored to our setting. For a general treatment of convex analysis we refer the reader to [ET99, Ber82, HUL93a, HUL93b].

In the following we call a function $\mathcal{F}: \mathbb{R}^{N} \rightarrow \mathbb{R} \cup\{\infty\}$ coercive if $\mathcal{F}(u) \stackrel{\|u\| \rightarrow \infty}{\longrightarrow}+\infty$ and lower-semicontinuous if $\liminf _{u \rightarrow u_{0}} \mathcal{F}(u) \geq \mathcal{F}\left(u_{0}\right)$ for all $u_{0} \in \mathbb{R}^{n}$.

Definition 2.2.1 ([ET99][p.20,Def.5.1]). Let $\mathcal{F}: \mathbb{R}^{n} \rightarrow \mathbb{R}$ be a function and $u_{0} \in \mathbb{R}^{n}$, then we define its sub-differential $\partial \mathcal{F}\left(u_{0}\right)$ at $u_{0}$ as the set

$$
u^{*} \in \partial \mathcal{F}\left(u_{0}\right) \quad \Leftrightarrow \quad\left\langle u-u_{0}, u^{*}\right\rangle \leq \mathcal{F}(u)-\mathcal{F}\left(u_{0}\right) \text { for all } u \in \mathbb{R}^{n}
$$

Proposition 2.2.2 ([ET99](p.38,Prop.2.2)). Let $\mathcal{F}=\mathcal{F}_{1}+\mathcal{F}_{2}: \mathbb{R}^{n} \rightarrow \mathbb{R}$ be a coercive functional, with $\mathcal{F}_{2}$ lower semi-continuous and convex. Furthermore, let $\mathcal{F}_{1}$ be convex 
and differentiable with gradient $D_{y} \mathcal{F}_{1}$ at a point $y \in \mathbb{R}^{n}$. Then we have

$$
u \in \arg \min _{u \in \mathbb{R}^{n}} \mathcal{F}(u)
$$

if and only if the following inequality holds

$$
\left\langle D_{u} \mathcal{F}_{1}, v-u\right\rangle+\mathcal{F}_{2}(v)-\mathcal{F}_{2}(u) \geq 0, \quad \forall v \in \mathbb{R}^{n}
$$

See the proof in [ET99][p.38,Prop.2.2], that we reproduced for convenience in Appendix A.2.

\subsection{Mapping Degree Theory}

Let us collect all notions and results from [OR09] about the degree of a continuous mapping and its interplay with homotopies. These results will be needed in Chapter 4.1.1. In this chapter $\Omega$ is a bounded, open and connected subset of $\mathbb{R}^{n}$. Furthermore, $\bar{\Omega}$ will denote its closure and $\Omega:=\bar{\Omega} \backslash \Omega$ its boundary (we abstain from the usual $\partial \Omega$, since $\partial$ is used for the subdifferential). We call a map $f: \bar{\Omega} \rightarrow \mathbb{R}^{n}$ differentiable if its Jacobian exists everywhere, define the norm $\|f\|_{\infty}:=\sup _{\omega \in \Omega} f(\omega)$ and call a point $\omega \in \Omega$ a regular value if its Jacobian is surjective. For proofs of the following propositions we refer the reader to Outerelo and Ruiz [OR09] and Jordan [Jor75], respectively.

Proposition and Definition 2.3.1 ([OR09], §IV, Prop. and Def. 1.1). Let $f: \bar{\Omega} \rightarrow \mathbb{R}^{n}$ be a differentiable mapping and $p \notin f(\Omega)$ a regular value, then the degree $\operatorname{deg}(f, \Omega, p)$ is defined as:

$$
\operatorname{deg}(f, \Omega, p):=\sum_{\omega \in f^{-1}(p)} \operatorname{sign}\left(\operatorname{det}\left(D_{\omega} f\right)\right)
$$

where the sum is 0 in case of $f^{-1}(p)=\emptyset$ and $D_{\omega} f$ is the Jacobian matrix at point $\omega \in \Omega$. The notion of degree is extended to continuous functions.

Proposition and Definition 2.3.2 ([OR09], §IV, Prop. and Def. 2.1). Let $f: \bar{\Omega} \rightarrow \mathbb{R}^{n}$ be a continuous mapping and $p \notin f(\Omega)$. Then there exists a differentiable mapping $g: \bar{\Omega} \rightarrow \mathbb{R}^{n}$ such that $\|f-g\|_{\infty} \leq d(p, f(\Omega))$ and $p$ is a regular value of $g$. For every such $g$ the degree $d(g, \Omega, p)$ is well-defined and independent of the choice of $g$, so we can define the degree with respect to $f$ as:

$$
\operatorname{deg}(f, \Omega, p):=\operatorname{deg}(g, \Omega, p)=\sum_{\omega \in g^{-1}(p)} \operatorname{sign}\left(\operatorname{det}\left(D_{\omega} g\right)\right)
$$


Lemma 2.3.3 ([OR09] §IV, Prop. 2.4). Let $f$ and $g$ be continuous mappings from $\bar{\Omega}$ to $\mathbb{R}^{n}$ and let $H:[0,1] \times \bar{\Omega} \rightarrow \mathbb{R}^{n}$ be a homotopy between $f$ and $g$, i.e. a continuous mapping such that $H(0, \cdot)=f$ and $H(1, \cdot)=g$, such that for all $t \in[0,1]$ we have $p \notin H(t, \stackrel{\Omega}{\Omega})$, then

$$
\operatorname{deg}(f, \Omega, p)=\operatorname{deg}(g, \Omega, p)
$$

Corollary 2.3.4 ([OR09], §IV, Cor. 2.5 (2)). Let $f: \bar{\Omega} \rightarrow \mathbb{R}^{n}$ be a continuous mapping and let $p \notin f(\Omega)$, if $\operatorname{deg}(f, \Omega, p) \neq 0$ then $p \in \operatorname{Im}(f)$.

The last ingredient we need later is the definition of principal angles between two linear subspaces, as defined for example in [Jor75, ZK13].

Proposition and Definition 2.3.5 ([Jor75, ZK13]). Let $\Xi$ and $\Omega$ be two linear subspaces of $\mathbb{R}^{n}$ with dimensions $n_{1}$ and $n_{2}$, respectively. Let $m=\min \left(n_{1}, n_{2}\right)$. Choose first two vectors $\xi_{1} \in \Xi$ and $\omega_{1} \in \Omega$ such that

$$
\begin{aligned}
& \left(\xi_{1}, \omega_{1}\right) \in \quad \arg \max \quad|\langle\xi, \omega\rangle|, \\
& \xi \in \Xi:\|\xi\|=1 \\
& \omega \in \Omega:\|\omega\|=1
\end{aligned}
$$

then choose recursively a set of vectors $\left\{\xi_{2}, \ldots, \xi_{m}\right\} \subset \Xi$ and $\left\{\omega_{2}, \ldots, \omega_{m}\right\} \subset \Omega$ by the relation

$$
\begin{aligned}
& \left(\xi_{k}, \omega_{k}\right) \in \quad \arg \max \quad|\langle\xi, \omega\rangle|, \\
& \xi \in \Xi \text { such that }\left\langle\xi, \xi_{j}\right\rangle=0 \text { for all } 1 \leq j \leq k-1 \text { and }\|\xi\|=1 \\
& \omega \in \Omega \text { such that }\left\langle\omega, \omega_{j}\right\rangle=0 \text { for all } 1 \leq j \leq k-1 \text { and }\|\omega\|=1
\end{aligned}
$$

for $2 \leq k \leq m$. We call the sets $\left\{\xi_{1}, \ldots, \xi_{m}\right\} \subset \Xi$ and $\left\{\omega_{1}, \ldots, \omega_{m}\right\} \subset \Omega$ the principal vectors. Then we define the principal angles between $\Xi$ and $\Omega$ as the set $\Theta(U, W)=$ $\left\{\theta_{1}, \ldots, \theta_{m}\right\}$ defined by

$$
\theta_{k}=\arccos \left(\left|\left\langle\xi_{k}, \omega_{k}\right\rangle\right|\right) \in\left[0, \frac{\pi}{2}\right] \text { for all } 1 \leq k \leq m
$$

The principal angles are independent of the choice of principal vectors in (2.3.3).

The notion is extended to affine linear subspaces that intersect in exactly one point.

Definition 2.3.6. Let $\Xi, \Omega$ be affine linear subspace of $\mathbb{R}^{n}$ that have a unique intersection point $v$. We define their principal vectors and principal angles by those of the linear subspaces

$$
\widetilde{\Xi}:=\left\{\xi-v \in \mathbb{R}^{n}: \xi \in \Xi\right\}
$$


and

$$
\widetilde{\Omega}:=\left\{\omega-v \in \mathbb{R}^{n}: \omega \in \Omega\right\} .
$$

\subsection{Set-Valued Theory}

In this chapter we give the basic definition of set-valued functional calculus, using the notions and definitions of Aubin and Ekeland [AE84]. We will sometimes omit the exact general statements in favour of more specific statements suited specifically for our setting. These definitions will be used mainly in Chapter 4.1.2.

First, we define the Hausdorff distance $d: 2^{\mathbb{R}^{n}} \times 2^{\mathbb{R}^{n}} \rightarrow \mathbb{R}_{+}$between two sets $\Xi, \Omega \subset \mathbb{R}^{n}$ by

$$
d(\Xi, \Omega):=\max \left(\sup _{\xi \in \Xi} \inf _{\omega \in \Omega}(\|\xi-\omega\|), \sup _{\omega \in \Omega} \inf _{\xi \in \Xi}(\|\xi-\omega\|)\right)
$$

Definition 2.4.1 ([AE84][p.1-3]). A set-valued map is a mapping $\Upsilon$ from a space $\mathbb{R}^{n}$ to a space $\mathbb{R}^{m}$ that associates with any $x \in \mathbb{R}^{n}$ a subset $\Upsilon(x) \subset \mathbb{R}^{m}$, called the image or value of $\Upsilon$ at $x$. This relation is commonly denoted by $\Upsilon: \mathbb{R}^{n} \rightrightarrows \mathbb{R}^{m}$. We further call

$$
\operatorname{Dom}(\Upsilon):=\left\{x \in \mathbb{R}^{n}: \Upsilon(x) \neq \emptyset\right\}
$$

the domain of $\Upsilon$. We say $\Upsilon$ is strict w.r.t. $\mathbb{R}^{n}$ if $\operatorname{Dom}(\Upsilon)=\mathbb{R}^{n}$.

Additionally, we define the graph of $\Upsilon$ to be

$$
\operatorname{Graph}(\Upsilon):=\left\{(x, y) \in \mathbb{R}^{n} \times \mathbb{R}^{m}: y \in \Upsilon(x)\right\}
$$

and the range of $\Upsilon$ by

$$
\Upsilon\left(\mathbb{R}^{n}\right):=\left\{y \in \mathbb{R}^{m}: \exists x \in \mathbb{R}^{n} \text { s.t. } y \in \Upsilon(x)\right\}
$$

We say a set-valued map $\Upsilon$ is closed-valued, convex-valued, bounded-valued or compactvalued if the images $\Upsilon(x)$ are closed, convex, bounded or compact for all $x \in \mathbb{R}^{n}$, respectively. We will say $\Upsilon$ is closed, convex, bounded or compact if its graph is closed, convex, bounded or compact, respectively.

Next, we introduce the notion of upper semi-continuity of [AE84][p.108].

Definition 2.4.2 ([AE84][p.108]). We say a set-valued map $\Upsilon: \mathbb{R}^{n} \rightrightarrows \mathbb{R}^{m}$ is upper semicontinuous (in short, u.s.c.) at $x_{0} \in \mathbb{R}^{n}$ if for any $\epsilon>0$ there exists a $\delta>0$ such 
that for all $x \in \mathbb{R}^{n}$ such that $\left\|x_{0}-x\right\|<\delta$ we have that

$$
\min _{z_{1} \in \Upsilon\left(x_{0}\right)}\left(\left\|z_{1}-z_{2}\right\|\right)<\epsilon \quad \text { for all } z_{2} \in \Upsilon(x)
$$

We say $\Upsilon$ is upper semicontinuous if $\Upsilon$ is u.s.c. at every point $x \in \mathbb{R}^{n}$. 


\section{CHAPTER 3}

\section{Constrained Double Minimization}

In the following chapter the proposed algorithm is motivated, set up and characterized via a variational problem, named constrained double minimization (CDM). We start by revisiting the classical $\ell^{1}$-regularization problems for $F \in \mathbb{R}^{n \times m}, \underline{B} \in \Gamma_{P}, \kappa \in\{1,2\}$ and $\mu \in \mathbb{R}_{+}$of (1.3.2), restated in the following

$$
\begin{aligned}
& \operatorname{minimize} \quad \mathcal{J}_{\min }(U):=\left|\underline{\mathfrak{C}}_{\underline{B}}(U)\right|_{1, \kappa}+\frac{\mu}{2}\|U-F\|^{2}, \\
& \text { over } \quad U \in \mathbb{R}^{n \times m} .
\end{aligned}
$$

In Chapter 3.1.1 equivalent problem formulations for (1.3.2) are discussed, see for an overview Figure 3.1. We highlight in Chapter 3.1.2 a drawback of models of the form (1.3.2) in order to motivative in Chapter 3.1.3 the further generalization of (1.3.2). The proposed algorithm is set-up in Chapter 3.2. Moreover, in Chapter 3.3 we derive a variational problem that motivates the name constrained double minimization, and gives a first interpretation of the solutions of the proposed algorithm.

\subsection{Revisiting $\ell^{1}$-Regularizations}

As depicted in Figure 3.1 the variational problem in (1.3.2) can be equivalently stated as a problem of finding a point in the intersection of multiple sets. We will make use of these equivalent notions to motivate and to state our proposed generalized algorithm in the following.

\subsubsection{Some Equivalent Problem Formulations}

Let us start with the classical discrete $T V-\ell^{2}$-model proposed by Rudin, Osher and Fatemi in [ROF92], discretized according to our notation of Chapter 2. As shown in Chambolle and Lions in [CL97] the model in [ROF92] can be rewritten as the non-constrained optimization problem given below. 


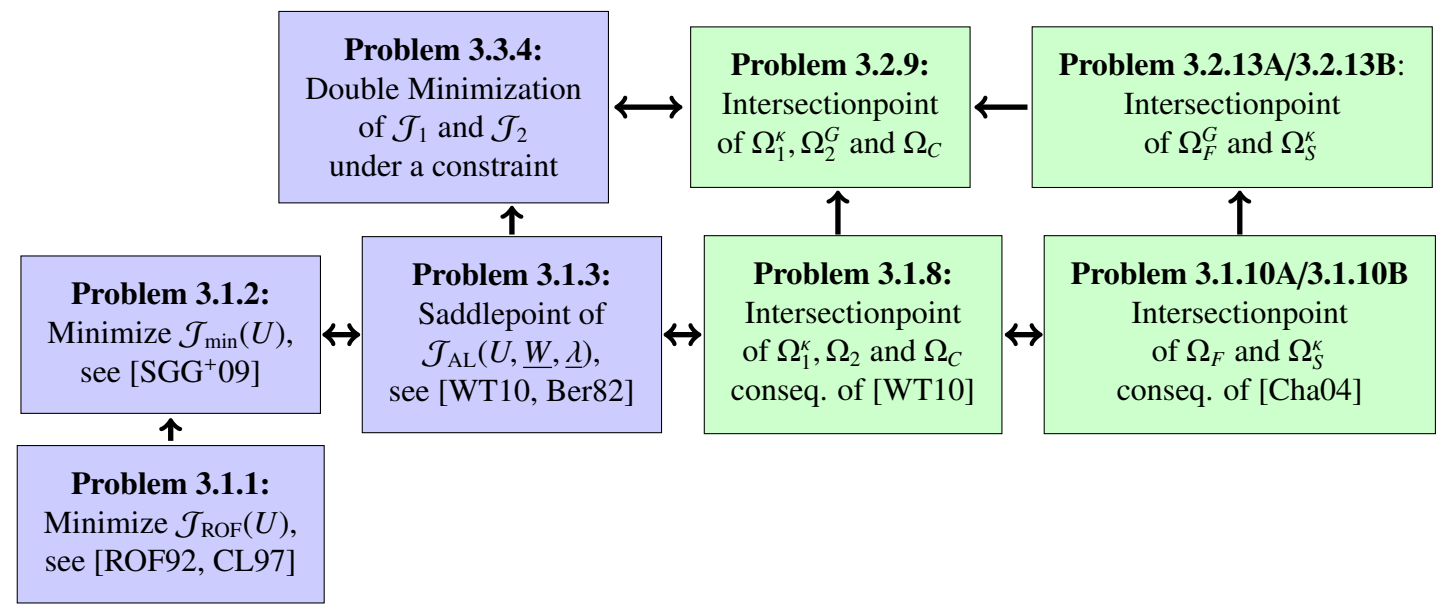

Figure 3.1: Overview over problem formulations in Chapter 3 (blue = variational problems, green $=$ intersection point problems). If there is an arrow from problem $A$ to problem $B$, then problem $A$ is a special case of problem $B$. Hence, a double arrow marks an equivalence.

Problem 3.1.1 ([CL97]). Let $F \in \mathbb{R}^{n \times m}$ be an input image, $\underline{D} \in \Gamma_{2}$ the discrete gradient matrix-family introduced in Example 2.1.12 and $\mu \in \mathbb{R}_{+}$. The (isotropic) $T V-\ell^{2}$-model is given by

$$
\begin{aligned}
& \operatorname{minimize} \mathcal{J}_{\mathrm{ROF}}(U):=\left|\underline{\mathfrak{C}}_{\underline{D}}(U)\right|_{1,2}+\frac{\mu}{2}\|U-F\|^{2}, \\
& \text { over } U \in \mathbb{R}^{n \times m} \text {. }
\end{aligned}
$$

As discussed in the introduction, the $T V-\ell^{2}$-model of Problem 3.1.1 is a special case of the broader class of $\ell^{1}$-regularization problems. For a general treatment of $\ell^{1}$-regularization techniques see for example Scherzer et al. [SGG $\left.{ }^{+} 09\right][\mathrm{p} .87 \mathrm{ff}]$.

Problem 3.1.2 ([SGG ${ }^{+}$09][p.87]). Let $F \in \mathbb{R}^{n \times m}$ be an input image, then for $\underline{B} \in \Gamma_{P}$, $\kappa \in\{1,2\}$ and $\mu \in \mathbb{R}_{+}$an $\ell^{1}$-regularization problem is given by

$$
\begin{aligned}
& \operatorname{minimize} \mathcal{J}_{\min }(U):=\left|\underline{\mathfrak{C}}_{\underline{B}}(U)\right|_{1, \kappa}+\frac{\mu}{2}\|U-F\|^{2}, \\
& \text { over } \quad U \in \mathbb{R}^{n \times m} .
\end{aligned}
$$

As detailed in Chapter 1.4.5 many algorithms have been proposed to solve Problem 3.1.1/3.1.2. In the following we will focus on the alternating direction method of multipliers (ADMM) for computing the saddle point of the augmented Lagrangian functional (for a general treatment of augmented Lagrangian methods see [ET99][p.54ff.] or [Ber82][p.95ff.]), proposed for the $T V-\ell^{2}$ problem by Wu and Tai in [WT10].

The augmented Lagrangian functional for $F, U \in \mathbb{R}^{n \times m} \underline{B} \in \Gamma_{P}, \mu, \beta \in \mathbb{R}_{+}, \kappa \in\{1,2\}$ and $\underline{W}, \underline{\lambda} \in \Gamma_{P}$ is given by 


$$
\mathcal{J}_{\mathrm{AL}}(U, \underline{W}, \underline{\lambda}):=|\underline{W}|_{1, \kappa}+\frac{\mu}{2}\|U-F\|^{2}+\frac{\beta}{2}\left\|\underline{W}-\underline{\mathfrak{C}}_{\underline{B}}(U)\right\|^{2}+\left\langle\underline{\lambda}, \underline{W}-\underline{\mathfrak{C}}_{\underline{B}}(U)\right\rangle .
$$

A saddle point of $J_{\mathrm{AL}}$ is then given by a point $\left(U^{\dagger}, \underline{W^{\dagger}}, \underline{\lambda}^{\dagger}\right) \in \Gamma_{1+2 P}$ satisfying

$$
\mathcal{J}_{\mathrm{AL}}\left(U^{\dagger}, \underline{W^{\dagger}}, \underline{\lambda}\right) \leq \mathcal{J}_{\mathrm{AL}}\left(U^{\dagger}, \underline{W^{\dagger}}, \underline{\lambda}^{\dagger}\right) \leq \mathcal{J}_{\mathrm{AL}}\left(U, \underline{W}, \underline{\lambda^{\dagger}}\right) \quad \text { for all }(U, \underline{W}, \underline{\lambda}) \in \Gamma_{1+2 P} .
$$

We obtain the saddle point problem given below.

Problem 3.1.3. [[WT10],[Ber82][p.96ff.]] Given an input image $F \in \mathbb{R}^{n \times m}, \underline{B} \in \Gamma_{P}, \mu \in$ $\mathbb{R}_{+}$and $\kappa \in\{1,2\}$, let the augmented Lagrangian $\mathcal{J}_{\mathrm{AL}}$ be defined as in (3.1.2) for some $\beta \in \mathbb{R}_{+}$. Find a saddle point $\left(U^{\dagger}, \underline{W}^{\dagger}, \underline{\lambda}^{\dagger}\right) \in \Gamma_{1+2 P}$ of $\mathcal{J}_{\mathrm{AL}}$.

Let us state the equivalence of Problem 3.1.2 and Problem 3.1.3. We give a proof in Appendix A.2, based on the proof of Wu and Tai [WT10][Theorem 4.1], who considered the case of $\underline{B}=\underline{D}$. For a general proof we refer the reader to Bertsekas [Ber82][p.96ff].

Lemma 3.1.4 ([WT10][Theorem 4.1],[Ber82][p.96ff]). Let $F \in \mathbb{R}^{n \times m}, \underline{B} \in \Gamma_{P}, \mu \in \mathbb{R}_{+}$ and $\kappa \in\{1,2\}$. Then $U^{\dagger} \in \mathbb{R}^{n \times m}$ is a solution of Problem 3.1.2 if and only if there exists $\underline{W}^{\dagger} \in \Gamma_{P}$ and $\underline{\lambda}^{\dagger} \in \Gamma_{P}$ such that $\left(U^{\dagger}, \underline{W^{\dagger}}, \underline{\lambda}^{\dagger}\right)$ is a saddlepoint of (3.1.2).

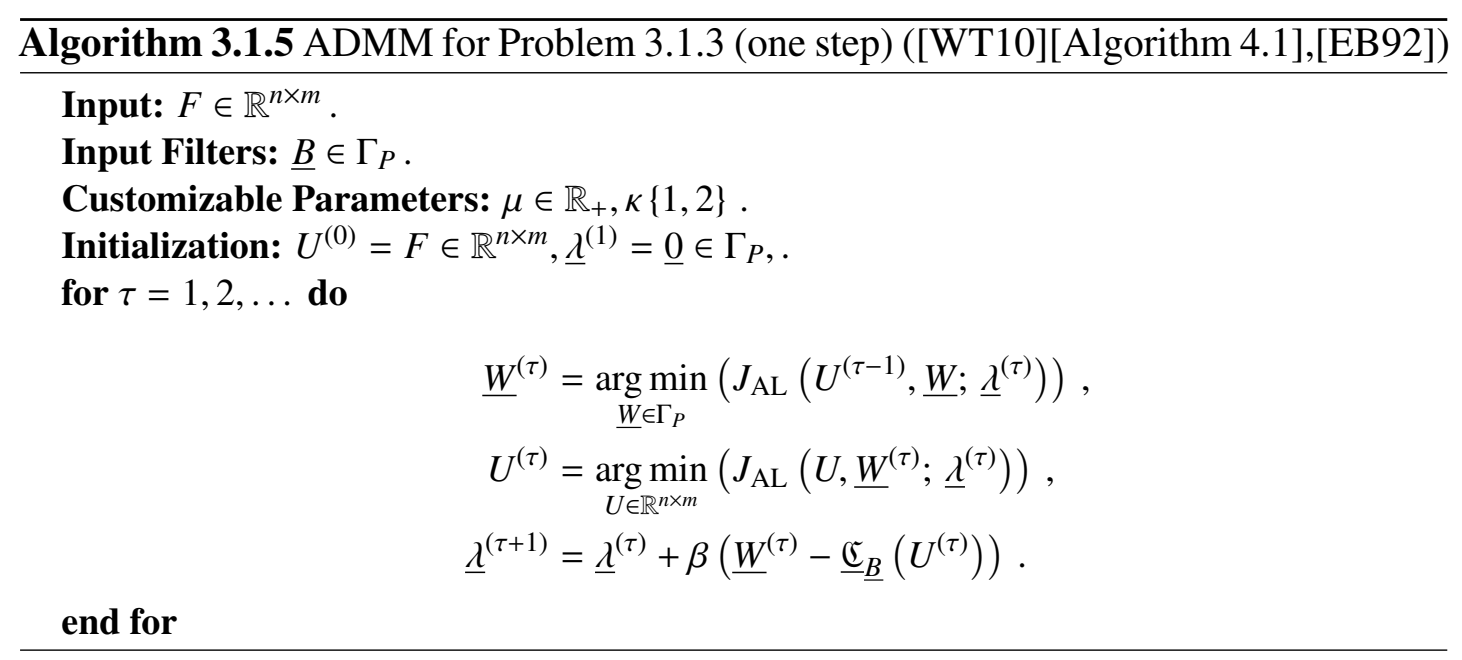

We present the algorithm of [WT10] for general $\underline{B} \in \Gamma_{P}$ and $\kappa \in\{1,2\}$ in Algorithm 3.1.5 solving Problem 3.1.3 (and Problem 3.1.2). Algorithm 3.1.5 is a well-known ADMM algorithm for finding the saddle-point of an augmented Lagrangian functional, we refer to Glowinski and Le Tallec [GLT89][Theorem 2.2] or Eckstein and Bertsekas [EB92][Theorem 8] for convergence results. For the special case of the $T V-\ell^{2}$-model in Problem 3.1.1 one might want to consider the proof in [WT10]. We 
only state here that a fixed point of Algorithm 3.1.5 is a solution of Problem 3.1.3, which is a direct consequence of Theorem 4.4 of [WT10] in the case of Problem 3.1.1.

Lemma 3.1.6 ([WT10][Theorem 4.4]). Let $F \in \mathbb{R}^{n \times m}, \underline{B} \in \Gamma_{P}, \kappa \in\{1,2\}$ and $\beta \in \mathbb{R}_{+}$. A point $\left(U^{\dagger}, \underline{W}^{\dagger}, \underline{\lambda}^{\dagger}\right) \in \Gamma_{1+2 P}$ is a fixed point of Algorithm 3.1 .5 if and only if $\left(U^{\dagger}, \underline{W}^{\dagger}, \underline{\lambda}^{\dagger}\right)$ is a saddlepoint of (3.1.2).

For completeness of presentation, we present a proof of Lemma 3.1.6 in Appendix A.2, adapting the proof of Theorem 4.4 of [WT10].

In the following Lemma 3.1.7 we state the minimizers of the updates in Algorithm 3.1.5 explicitly. In Appendix A.2 we reproduce their derivation, following standard variational calculus, see [BC11, HUL93a, HUL93b, BV04], or for the special case of $T V-\ell^{2}$ [WT10, CGP09].

Lemma 3.1.7 ([BC11],[BV04]). (i) For a given $U \in \mathbb{R}^{n \times m}, \underline{B}, \underline{\lambda} \in \Gamma_{P}, \kappa \in\{1,2\}$, as well as $\beta \in \mathbb{R}_{+}$, the minimizer of

$$
\mathcal{J}_{1}(\underline{W}):=|\underline{W}|_{1, K}+\frac{\beta}{2} \| \underline{W}-\left.\underline{\mathfrak{c}}_{\underline{B}}(U)\right|^{2}+\langle\underline{\lambda}, \underline{W}\rangle,
$$

is given by

$$
\underline{W}^{\dagger}=\mathrm{S}_{\kappa}\left(\underline{\mathfrak{c}}_{\underline{B}}(U)-\frac{1}{\beta} \underline{\lambda} ; \frac{1}{\beta}\right),
$$

where $\mathrm{S}_{\kappa}: \Gamma_{P} \rightarrow \Gamma_{P}$ is the isotropic $(\kappa=2)$ or anisotropic $(\kappa=1)$ soft-shrinkage function, from Definition 2.1.17.

(ii) For a given $F \in \mathbb{R}^{n \times m}, \underline{B}, \underline{W}, \underline{\lambda} \in \Gamma_{P}$, and $\beta \in \mathbb{R}_{+}$, the minimizer of

$$
\mathcal{J}_{2}(U):=\frac{\mu}{2}\|F-U\|^{2}+\frac{\beta}{2}\left\|\underline{W}-\underline{\mathfrak{C}}_{\underline{B}}(U)\right\|^{2}-\left\langle\underline{\lambda}, \underline{\mathfrak{C}}_{\underline{B}}(U)\right\rangle,
$$

is given by

$$
U^{\dagger}=\mu\left(\mu \mathfrak{E}+\beta\left(\underline{\mathfrak{C}}_{\underline{B}}^{*} \underline{\mathbb{C}} \underline{B}\right)\right)^{-1}(F)+\beta\left(\mu \mathfrak{E}+\beta\left(\underline{\mathfrak{C}}_{\underline{B}}^{*} \underline{\mathfrak{C}} \underline{B}\right)\right)^{-1} \underline{\mathfrak{C}}_{\underline{B}}^{*}\left(\underline{W}+\frac{1}{\beta} \underline{\lambda}\right) .
$$

Given Algorithm 3.1.5 solving Problem 3.1.3 and Lemma 3.1.7 we state the intersection point problem below.

Problem 3.1.8. Given $F \in \mathbb{R}^{n \times m}, \underline{B} \in \Gamma_{P}, \mu \in \mathbb{R}_{+}$and $\kappa \in\{1,2\}$, and let $\beta \in \mathbb{R}_{+}$be arbitrary. Find a point $\left(U^{\dagger}, \underline{W^{\dagger}}, \underline{\lambda}^{\dagger}\right) \in \Gamma_{1+2 P}$ in the intersection of the following three 
sets

$$
\begin{aligned}
& \Omega_{1}^{\kappa}:=\left\{(U, \underline{W}, \underline{\lambda}) \in \Gamma_{1+2 P}: \underline{W}=\mathrm{S}_{K}\left(\underline{\mathfrak{C}}_{\underline{B}}(U)-\frac{1}{\beta} \underline{\lambda} ; \frac{1}{\beta}\right)\right\}, \\
& \Omega_{2}:=\left\{(U, \underline{W}, \underline{\lambda}) \in \Gamma_{1+2 P}:\right. \\
& \left.\quad U=\mu\left(\mu \mathfrak{\mathbb { E }}+\beta \underline{\mathfrak{C}}_{\underline{\underline{C}}}^{*} \underline{\mathfrak{C}}_{\underline{B}}\right)^{-1}(F)+\beta\left(\mu \mathfrak{\mathbb { E }}+\beta \underline{\mathfrak{C}}_{\underline{B}}^{*} \underline{\mathfrak{C}}_{\underline{B}}\right)^{-1} \underline{\mathfrak{C}}_{\underline{B}}^{*}\left(\underline{W}+\frac{1}{\beta} \underline{\lambda}\right)\right\}, \\
& \Omega_{C}:=\left\{(U, \underline{W}, \underline{\lambda}) \in \Gamma_{1+2 P}: \underline{\mathfrak{C}}_{\underline{B}}(U)=\underline{W}\right\} .
\end{aligned}
$$

Lemma 3.1.6 and Lemma 3.1.7 yield at once the following Corollary.

Corollary 3.1.9. Given $F \in \mathbb{R}^{n \times m}, \underline{B} \in \Gamma_{P}, \kappa \in\{1,2\}$ and $\mu \in \mathbb{R}_{+}$, let $\beta \in \mathbb{R}_{+}$be arbitrary. A point $\left(U^{\dagger}, \underline{W}^{\dagger}, \underline{\lambda}^{\dagger}\right) \in \Gamma_{1+2 P}$ is in the intersection of $\Omega_{1}^{\kappa}, \Omega_{2}$ and $\Omega_{C}$ if and only if it is a saddlepoint of (3.1.2).

Proof. Let $\left(U^{\dagger}, \underline{W}^{\dagger}, \underline{\lambda}^{\dagger}\right) \in \Omega_{1}^{\kappa} \cap \Omega_{2} \cap \Omega_{C}$, then $\underline{W}^{\dagger}$ is by (i) of Lemma 3.1.7 a solution of $\mathcal{J}_{\mathrm{AL}}$ for fixed $U^{\dagger}$ and $\underline{\lambda}^{\dagger}$. Moreover, $U^{\dagger}$ is by (ii) of Lemma 3.1.7 a solution of $\mathcal{J}_{\mathrm{AL}}$ for fixed $\underline{W}^{\dagger}$ and $\underline{\lambda}^{\dagger}$. Finally, by $\left(U^{\dagger}, \underline{W}^{\dagger}, \underline{\lambda}^{\dagger}\right) \in \Omega_{C}$ we have

$$
\underline{\lambda}^{\dagger}=\underline{\lambda}^{\dagger}+\beta\left(\underline{W}^{\dagger}-\underline{\underline{C}}_{\underline{B}}\left(U^{\dagger}\right)\right),
$$

hence, $\left(U^{\dagger}, \underline{W^{\dagger}}, \underline{\lambda^{\dagger}}\right)$ is a fixed point of Algorithm 3.1.5.

Conversely if $\left(U^{\dagger}, \underline{W}^{\dagger}, \underline{\lambda}^{\dagger}\right)$ is a fixed point of Algorithm 3.1.5 we can reverse the above arguments to obtain $\left(U^{\dagger}, \underline{W}^{\dagger}, \underline{\lambda}^{\dagger}\right) \in \Omega_{1}^{\kappa} \cap \Omega_{2} \cap \Omega_{C}$.

Via Problem 3.1.8 we state a second intersection problem, split in two cases for $\kappa=1$ and $\kappa=2$, respectively. Note that Problem 3.1.10A/3.1.10B below is closely related to the dual formulation of Problem 3.1.2 in [Cha04]. In particular Equation (3.1.9), below, is a discrete version of the continuous Equation (7) in [Cha04].

Problem 3.1.10A. Let $F \in \mathbb{R}^{n \times m}, \underline{B} \in \Gamma_{P}$ and $\mu \in \mathbb{R}_{+}$. Find a point $\left(U^{\dagger}, \underline{\lambda}^{\dagger}\right) \in \Gamma_{1+P}$ in the intersection of the following two sets:

$$
\Omega_{S}^{1}:=\left\{(U, \underline{\lambda}) \in \Gamma_{1+P}: \lambda_{p}[k, \ell]\left\{\begin{array}{ll}
=-1 & \text { if } \mathfrak{c}_{B_{p}}(U)[k, \ell]>0 \\
\in[-1,1] & \text { if } \mathfrak{C}_{B_{p}}(U)[k, \ell]=0, \text { for all } k, \ell, p \\
=1 & \text { if } \mathfrak{C}_{B_{p}}(U)[k, \ell]<0
\end{array}\right\},\right.
$$

and

$$
\Omega_{F}:=\left\{(U, \underline{\lambda}) \in \Gamma_{1+P}: U=F+\mu^{-1} \underline{\mathfrak{C}}_{\underline{B}}^{*}(\underline{\lambda})\right\} .
$$


Problem 3.1.10B. Let $F \in \mathbb{R}^{n \times m}, \underline{B} \in \Gamma_{P}$ and $\mu \in \mathbb{R}_{+}$. Find a point $\left(U^{\dagger}, \underline{\lambda}^{\dagger}\right) \in \Gamma_{1+P}$ in the intersection of the following two sets:

$$
\begin{aligned}
\Omega_{S}^{2}:=\{ & (U, \underline{\lambda}) \in \Gamma_{1+P}: \\
& \left(\lambda_{p}[k, \ell]\right)_{p=1}^{P}\left\{\begin{array}{ll}
=-\frac{\left(\mathfrak{C}_{B_{p}}(U)[k, \ell]\right)_{p=1}^{P}}{\left\|\left(\mathfrak{C}_{B_{p}}(U)[k, \ell]\right)_{p=1}^{P}\right\|} & \text { if }\left\|\left(\mathfrak{C}_{B_{p}}(U)[k, \ell]\right)_{p=1}^{P}\right\|>0 \\
\in \mathcal{B}_{1}(0) & \text { if }\left\|\left(\mathfrak{C}_{B_{p}}(U)[k, \ell]\right)_{p=1}^{P}\right\|=0
\end{array}, \text { for all } k, \ell\right\},
\end{aligned}
$$

where $\mathcal{B}_{1}(0)$ is the ball of radius 1 around $0 \in \mathbb{R}^{P}$, and

$$
\Omega_{F}:=\left\{(U, \underline{\lambda}) \in \Gamma_{1+P}: U=F+\mu^{-1} \underline{\mathfrak{C}}_{\underline{B}}^{*}(\underline{\lambda})\right\} .
$$

The equivalence of Problem 3.1.8 and Problem 3.1.10A, Problem 3.1.10B, for $\kappa=1,2$, respectively, is a direct consequence of Chambolle's work in [Cha04][§2,§3, The.3.1]. Let us reproduce this result for our setting in the following Lemma 3.1.11.

Lemma 3.1.11 ([Cha04][§2, $\S 3$,The.3.1]). Let $F \in \mathbb{R}^{n \times m}, \underline{B} \in \Gamma_{P}$ and $\mu \in \mathbb{R}_{+}$.

(i) For $\kappa=1$, a point $\left(U^{\dagger}, \underline{\lambda}^{\dagger}\right) \in \Gamma_{1+P}$ is a solution of Problem 3.1.10A if and only if there exists $\underline{W}^{\dagger} \in \Gamma_{P}$ such that $\left(U^{\dagger}, \underline{W}^{\dagger}, \underline{\lambda}^{\dagger}\right)$ is a solution of Problem 3.1.8.

(ii) For $\kappa=2$, a point $\left(U^{\dagger}, \underline{\lambda}^{\dagger}\right) \in \Gamma_{1+P}$ is a solution of Problem 3.1.10B if and only if there exists $\underline{W}^{\dagger} \in \Gamma_{P}$ such that $\left(U^{\dagger}, \underline{W}^{\dagger}, \underline{\lambda}^{\dagger}\right)$ is a solution of Problem 3.1.8.

Proof. This proof is a corollary from the results presented in [Cha04][§2,§3, The.3.1]. Let $\left(U_{\kappa}^{\dagger}, \underline{\lambda}_{k}^{\dagger}\right) \in \Gamma_{1+P}$ be a solution of Problem 3.1.10A $(\kappa=1)$ or of Problem 3.1.10B $(\kappa=2)$. Choose $\underline{W}_{\kappa}^{\dagger}:=\underline{\mathfrak{c}}_{\underline{D}}\left(U_{\kappa}^{\dagger}\right)$, then we have by construction $\left(U_{\kappa}^{\dagger}, \underline{W}_{\kappa}^{\dagger}, \underline{\lambda}_{\kappa}^{\dagger}\right) \in \Omega_{C}$, for $\kappa=1,2$. Define

$$
\mathrm{A}_{\kappa}(\underline{W}):=|\underline{W}|_{1, \kappa}+\left\langle\underline{\lambda_{\kappa}^{\dagger}}, \underline{W}\right\rangle,
$$

then we have for both choices of $\kappa$ by the derivation of the subdifferential of $A_{\kappa}$, see [BC11] or the proof of Lemma 3.1.7 in Appendix A.2, that

$$
\underline{0} \in \partial \mathrm{A}_{\kappa}\left(\underline{\mathfrak{C}}_{\underline{B}}\left(U_{\kappa}^{\dagger}\right)\right) .
$$

Since we have by Proposition 16.34 in [BC11][p.233] that

$$
\underline{\mathfrak{C}}_{\underline{B}}\left(U_{K}^{\dagger}\right)=\operatorname{prox}_{1 / \beta A_{\kappa}}\left(\underline{\mathfrak{C}}_{\underline{B}}\left(U_{\kappa}^{\dagger}\right)\right) \quad \Leftrightarrow \quad \underline{0} \in \frac{1}{\beta} \mathrm{A}_{\kappa}\left(\underline{\mathfrak{C}}_{\underline{B}}\left(U_{\kappa}^{\dagger}\right)\right),
$$


Equation (3.1.11) yields $\left(U_{\kappa}^{\dagger}, \underline{W}_{\kappa}^{\dagger}, \underline{\lambda}_{\kappa}^{\dagger}\right) \in \Omega_{1}^{\kappa}$, for $\kappa=1,2$.

Moreover, we have for $\kappa \in\{1,2\}$ that

$$
\begin{aligned}
& U_{\kappa}^{\dagger}=F+\mu^{-1} \underline{\mathfrak{C}}_{\underline{B}}^{*}\left(\underline{\lambda}_{\kappa}^{\dagger}\right) \\
& \Leftrightarrow \mu\left(\mu \mathfrak{\mathbb { E }}+\beta \underline{\mathfrak{C}}_{\underline{B}}^{*} \underline{\mathfrak{C}}_{\underline{B}}\right)^{-1}\left(U_{\kappa}^{\dagger}\right)=\mu\left(\mu \mathfrak{E}+\beta \underline{\mathfrak{C}}_{\underline{B}}^{*} \underline{\mathfrak{C}}_{\underline{B}}\right)^{-1}(F) \\
& +\left(\mu \mathfrak{\mathbb { E }}+\beta \underline{\mathbb{C}}_{\underline{B}}^{*} \underline{\mathbb{C}} \underline{\underline{B}}\right)^{-1} \underline{\mathfrak{C}}_{\underline{B}}^{*}\left(\underline{\lambda}_{\kappa}^{\dagger}\right), \\
& \Leftrightarrow\left(\mathfrak{E}-\left(\mu \mathfrak{\mathbb { E }}+\beta \underline{\mathbb{C}}_{\underline{B}}^{*} \underline{\mathfrak{C}}_{\underline{B}}\right)^{-1} \beta \underline{\mathbb{C}}_{\underline{B}}^{*} \underline{\mathfrak{C}}_{\underline{B}}\right)\left(U_{\kappa}^{\dagger}\right)=\mu\left(\mu \mathfrak{\mathbb { E }}+\beta \underline{\mathbb{C}}_{\underline{B}}^{*} \underline{\mathfrak{C}}_{\underline{B}}\right)^{-1}(F) \\
& +\left(\mu \tilde{\mathfrak{E}}+\beta \underline{\mathfrak{C}}_{\underline{B}}^{*} \underline{\mathfrak{C}}_{\underline{B}}\right)^{-1} \underline{\mathfrak{C}}_{\underline{B}}^{*}\left(\underline{\lambda}_{K}^{\dagger}\right),
\end{aligned}
$$

where the inverse of $\left(\mu \mathfrak{E}+\beta \underline{\mathfrak{C}}_{B}^{*} \underline{\mathfrak{C}}_{B}\right)$ exists since $\underline{\mathfrak{C}}_{B}^{*} \underline{\mathfrak{C}}_{B}$ is a self-adjoint, positive semidefinite operator. Yielding,

$$
\Leftrightarrow U_{\kappa}^{\dagger}=\mu\left(\mu \mathfrak{\mathbb { E }}+\beta \underline{\mathfrak{C}}_{\underline{B}}^{*} \underline{\mathfrak{C}_{B}}\right)^{-1}(F)+\beta\left(\mu \mathfrak{E}+\beta \underline{\mathfrak{C}}_{\underline{B}}^{*} \underline{\mathfrak{C}}_{\underline{B}}\right)^{-1} \underline{\mathfrak{C}}_{\underline{B}}^{*}\left(\underline{W}_{\kappa}^{\dagger}-\frac{1}{\beta} \underline{\lambda}_{\kappa}^{\dagger}\right),
$$

and hence, $\left(U_{\kappa}^{\dagger}, \underline{W}_{\kappa}^{\dagger}, \underline{\lambda}_{k}^{\dagger}\right) \in \Omega_{2}$, for $\kappa=1,2$. The converse statement follows by reversing the above calculations.

\subsubsection{Loss of Contrast in the $T V-\ell^{2}$-Model}

Let us consider for $\kappa=2$ and $\underline{B}=\underline{D}$, the $T V-\ell^{2}$-model in Problem 3.1.1 and its equivalent problem formulations 3.1.2, 3.1.3, 3.1.8 and 3.1.10B. We consider the case of cartoon-texture-decompositions as done for example in Aujol et al. [AGCO06]. In this case the computed solutions $U^{\dagger}$ of Problem 3.1.1 should remove all small-scale oscillating patterns while keeping edges of large scale structures. A well known limitation of the $T V-\ell^{2}$-model is its loss of contrast, addressed for example in [Nik04, CE05]. In the case of cartoon-texture decomposition, loss-of-contrast can result in the failure to keep small edges, when highly oscillating textures are to be removed. We illustrate this via the toy example of Figure 3.2. Let us make an observation why we can not achieve both - keeping low edges and removing high oscillations - via Problem 3.1.10A/3.1.10B.

Consider the horizontal edge in the left-hand-side of (a) in Figure 3.2. Say, we want to completely preserve it in our solution $U^{\dagger}$. By (3.1.10) we have

$$
U^{\dagger}[k, \ell]=F[k, \ell]+\mu^{-1} \underline{\mathfrak{C}}_{\underline{D}}^{*}\left(\underline{\lambda}^{\dagger}\right)[k, \ell],
$$

for $k, \ell=1,2, \ldots, 128$. If $\underline{\mathfrak{C}}_{\underline{D}}^{*}\left(\underline{\lambda}^{\dagger}\right)[k, \ell]=0$ then the chosen $\mu$ has no influence on preserving the edge. If, however, $\underline{\mathfrak{C}}_{\underline{D}}^{*}\left(\underline{\lambda}^{\dagger}\right)[k, \ell] \neq 0$, then in order to keep the edge $\mu$ 


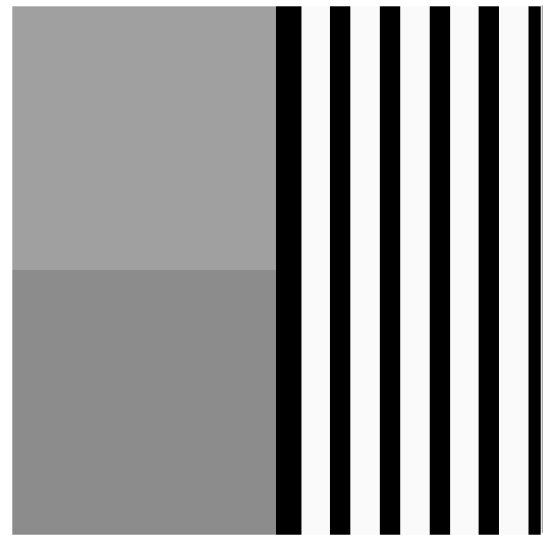

(a)

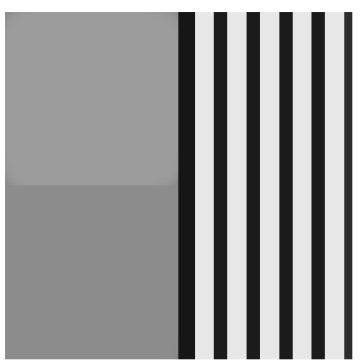

(b)

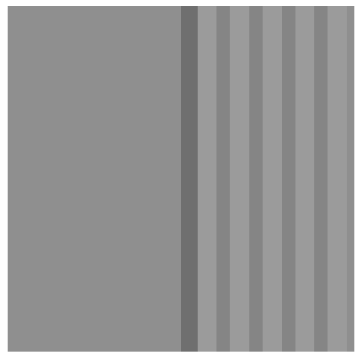

(d)

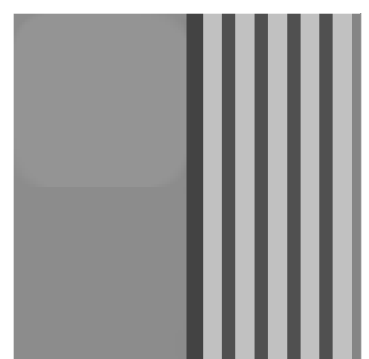

(c)

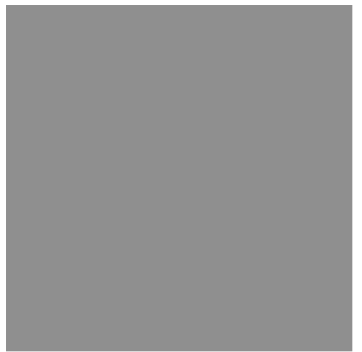

(e)

Figure 3.2: $128 \times 128$-pixel test image (a) that features a horizontal edge with height 20 and vertical texture with height 250 (for grey levels from 0 to 255), we present the solution $U^{\dagger}$ of Problem 3.1 .1 with $\mu=0.015$ (b), $\mu=0.005$ (c), $\mu=0.003$ (d) and $\mu=0.0005$ (e). Most instructive is (d) in which the texture on the right-hand-side has been only partially removed while the edge information on the left-hand-side is lost. 
has to be sufficiently big.

Turning to the vertical oscillating pattern on the right-hand-side of (b). Say, we want to remove it. In this case we observe via (3.1.9) that

$$
\left(\lambda_{p}^{\dagger}[k, \ell]\right)_{p=1}^{2} \in \mathcal{B}_{1}\left((0,0)^{T}\right)
$$

for all $k, \ell=1,2, \ldots, 128$. Using now (3.1.10) yields

$$
U[k, \ell]-F[k, \ell] \in \mathcal{B}_{2 \mu^{-1}}(0)
$$

for all $k, \ell=1,2, \ldots, 128$. Thus, to cancel texture patterns that are highly-oscillating as in right-hand-side of (a) in Figure 3.2 we need to choose $\mu$ sufficiently small.

Putting both observations together and considering (d) of Figure 3.2 we deduce that choosing $\mu=0.003$ in Problem 3.1.1 is too small to keep the horizontal edge, but still too large to remove the vertical texture completely. However, choosing a larger $\mu$ as in (b) and (c) will not remove the vertical pattern, while a smaller $\mu$ as in (e) will not recover the horizontal edge. That this observation occurs in practice can be seen for the barbara image considered in the numerical analysis in Chapter 5.2, see in particular Figure 5.7.

\subsubsection{Motivation of the Proposed Generalization}

To overcome loss of contrast it does not suffice to generalize to arbitrary $\underline{B} \in \Gamma_{P}$ and $\kappa \in\{1,2\}$, since both lead to Problem 3.1.10A/3.1.10B and thus, to the observation described in Chapter 3.1.2. There have been several extensions proposed in the literature tackling (among others) loss of contrast, cf. Chapter 1.4. A prominent approach consists of changing the data-fidelity norm in Problem 3.1.2, replacing the $\ell^{2}$-norm by the $\ell^{1}$-norm, e.g. Chan and Esedoglu [CE05], a G-norm, e.g. Meyer [Mey01] and Osher et al. [OSV03], or imposing local noise-constraints, e.g. Almansa et al. [ACHR06]. These show improvement when dealing with loss of contrast, see [AGCO06, CE05]. While a method embedding different norms for the data-fidelity term in a general algorithm via continuous parameters is, to the best of the author's knowledge, not available, for some of the proposed changes in the data-fidelity term it suffices to replace the balancing parameter $\mu$ by a matrix convolution while keeping the $\ell^{2}$-norm ([OSV03, AG06, GLMV07]). Moreover, approaches that directly consider an adaptive choice of $\mu \in \mathbb{R}_{+}$, replacing $\mu$ by a matrix $M \in \mathbb{R}^{n \times m}$ acting via component-wise multiplication or matrix-convolution, have been proposed, for example in Buades et 
al. [BLMV10] and Bredies et al. [BDH13].

Introducing a matrix convolution as a balancing term tackles directly the observation described in Chapter 3.1.2 by changing the set $\Omega_{F}$ in (3.1.8)/(3.1.10). We propose to generalize $\Omega_{F}$ in a broader way by replacing $\underline{B}$ in (3.1.8)/(3.1.10) with a new matrix-family convolution. Via Lemma 3.1.11 we can frame this as a generalization of Problem 3.1.8. Since Problem 3.1.8 is defined via the fixed point set of Algorithm 3.1.5, when generalizing Problem 3.1.8, we can infer in a straight-forward way a generalized algorithm. In Chapter 4.2.1 we draw the connection of the general model, proposed in this thesis, to the models of [OSV03, AG06, GLMV07, BLMV10, BDH13], some of which turn out to be special cases.

Idea 3.1.12. Generalize the classical $\ell^{1}$-regularization by changing $\Omega_{2}$ in Problem 3.1 .8 to

$$
\Omega_{2}^{G}:=\left\{(U, \underline{W}, \underline{\lambda}) \in \Gamma_{1+2 P}: U=\mathfrak{C}_{A}(F)+\beta \underline{\mathfrak{C}}_{\underline{\underline{B}}}^{*}\left(\underline{W}+\frac{1}{\beta} \underline{\lambda}\right)\right\},
$$

for some $A \in \mathbb{R}^{n \times m}$ and $\underline{\widetilde{B}} \in \Gamma_{P}$, different from $\underline{B}$.

\subsection{The Generalized Algorithm}

Based on Idea 3.1.12 we formalize a generalized cartoon-residual decomposition algorithm.

\subsubsection{Input Filters}

We characterize possible inputs $(A, \underline{B}, \underline{\widetilde{B}})$ in the spirit of Idea 3.1.12 and show some auxiliary results.

Definition 3.2.1. Let $A \in \mathbb{R}^{n \times m}$ and $\underline{B}=\left(B_{p}\right)_{p=1}^{P} \in \Gamma_{P}$ as well as $\underline{\widetilde{B}}=\left(\widetilde{B}_{p}\right)_{p=1}^{P} \in \Gamma_{P}$, then we call a triple $(A, \underline{B}, \underline{\widetilde{B}})$ input filters for Algorithm 3.2.8. We furthermore introduce the following conditions on $(A, \underline{B}, \underline{\widetilde{B}})$ and $(\underline{B}, \underline{\widetilde{B}})$, respectively.

(i) We say that $(\underline{B}, \underline{\widetilde{B}})$ are weakly admissible if for all $1 \leq p \leq P$ and $0 \leq k \leq$ $n-1$ and $0 \leq \ell \leq m-1$ we have

$$
\widehat{\widetilde{B}}_{p}[k, \ell]=\widehat{Y}_{p}[k, \ell] \widehat{B}_{p}[k, \ell],
$$

for some $\underline{Y}=\left(Y_{p}\right)_{p=1}^{P} \in \Gamma_{P}$ with $\widehat{Y}_{p} \in \mathbb{R}_{+}^{n \times m}$, called admissibility matrix-family. 
(ii) We say that $(\underline{B}, \underline{\widetilde{B}})$ are strongly admissible if for all $1 \leq p \leq P$ and $0 \leq k \leq$ $n-1$ and $0 \leq \ell \leq m-1$ we have

$$
\widehat{\widetilde{B}}_{p}[k, \ell]=\widehat{Y}[k, \ell] \widehat{B}_{p}[k, \ell]
$$

for some $Y \in \mathbb{R}^{n \times m}$ with $\widehat{Y} \in \mathbb{R}_{+}^{n \times m}$, called strong admissibility matrix.

(iii) We say that $(\underline{B}, \underline{\widetilde{B}})$ satisfy the non-expansive and positive semidefinite condition (NEPC) if

$$
0 \leq \sum_{p=1}^{P} \overline{\widehat{\widetilde{B}}_{p}[k, \ell]} \widehat{B}_{p}[k, \ell] \leq 1, \quad \text { for all } 0 \leq k \leq n-1 \text { and } 0 \leq \ell \leq m-1
$$

(NEPC)

Furthermore, we say $(A, \underline{B}, \underline{\widetilde{B}})$ satisfy strongly the (NEPC) if $(\underline{B}, \underline{\widetilde{B}})$ satisfy the (NEPC) and

$$
\sum_{p=1}^{P} \widehat{\widetilde{B}}_{p}[k, \ell] \widehat{B}_{p}[k, \ell]=1 \Rightarrow \widehat{A}[k, \ell]=0,
$$

for all $0 \leq k \leq n-1$ and $0 \leq \ell \leq m-1$.

(iv) We say that $(\underline{B}, \underline{\widetilde{B}})$ satisfy the contraction and positive semidefinite condition (CPC) if

$$
0 \leq \sum_{p=1}^{P} \overline{\widehat{\widetilde{B}}_{p}[k, \ell]} \widehat{B}_{p}[k, \ell]<1, \quad \text { for all } 0 \leq k \leq n-1 \text { and } 0 \leq \ell \leq m-1 \text {. (CPC) }
$$

Remark 3.2.2. While the term admissibility is newly introduced here, the terms positive semidefinite, non-expansiveness and contraction are chosen because they relate to the respective properties of the operator $\underline{\mathbb{C}}_{\widetilde{B}}^{*} \underline{\mathfrak{C}}_{\underline{B}}$. Under both (NEPC) and $(C P C)$ the operator $\underline{\mathfrak{E}}_{\widetilde{B}}^{*} \underline{\mathfrak{C}}_{\underline{B}}$ is semidefinite. Moreover, under the (NEPC) the eigenvalues of $\underline{\mathfrak{C}}_{\widetilde{\underline{B}}}^{*} \underline{\mathfrak{C}_{B}}$ are all less or equal to 1 , while under the $(C P C)$ they are strictly less than one, coining the notions of non-expansiveness and contraction condition.

Remark 3.2.3. For simplicity of presentation we work in the following with the straightforward and already very general condition of weak admissibility, even though many of the results can be shown to hold for slightly weaker, but more technical conditions, for example that the zero-entries of $\widehat{B}_{p}$ and $\widehat{\widetilde{B}}_{p}$ coincide for all $1 \leq p \leq P$.

Let us state further remarks and an auxiliary Lemma, that will be useful in Chapter 4.

Remark 3.2.4. (i) If $(\underline{B}, \underline{\widetilde{B}})$ is strongly admissible then its is also weakly admissible. 
(ii) If $(\underline{B}, \underline{\widetilde{B}})$ satisfies the $(C P C)$ then it strongly satisfies the (NEPC).

Lemma 3.2.5. Let $(\underline{B}, \underline{\widetilde{B}}) \in \Gamma_{2 P}$ be strongly admissible with $\underline{B}=\left(B_{p}\right)_{p=1}^{P}$ and $\underline{\widetilde{B}}=$ $\left(\widetilde{B}_{p}\right)_{p=1}^{P}$. Then, the linear operators

$$
\underline{\mathfrak{C}}_{\underline{\underline{B}}}^{*} \underline{\mathfrak{C}}_{\underline{B}}: \mathbb{C}^{n \times m} \rightarrow \mathbb{C}^{n \times m} \text { and } \underline{\mathfrak{C}}_{\underline{B}} \underline{\mathfrak{C}_{\widetilde{B}}^{*}}:\left(\mathbb{C}^{n \times m}\right)^{P} \rightarrow\left(\mathbb{C}^{n \times m}\right)^{P}
$$

are

(i) self-adjoint, and, thus diagonalizable by unitary operators defined on $\mathbb{C}^{n \times m}$ and $\left(\mathbb{C}^{n \times m}\right)^{P}$, respectively.

(ii) Furthermore, their set of non-zero eigenvalues coincides and is given by the following set of real, positive numbers

$$
\left\{\sum_{p=1}^{P}\left(\overline{\widetilde{\widetilde{B}}}_{p}[k, \ell] \widehat{B}_{p}[k, \ell]\right): \quad 0 \leq k \leq n-1 \text { and } 0 \leq \ell \leq m-1\right\} \backslash\{0\} .
$$

Proof. We begin by showing that $\underline{\mathbb{C}}_{\widetilde{B}}^{*} \underline{\mathfrak{C}}_{\underline{B}}$ is diagonalized by the unitary DFT operator $\frac{1}{\sqrt{n m}} \mathfrak{F}$, see Definition 2.1.6, which is derived by (iii) of Lemma 2.1.8, see also Stein and Shakarchi [SS03][p.218ff.].

Let $E_{r, s}$ be the matrix-entries of $\mathbb{E}$, see (i) of Definition 2.1.2, i.e. $E_{r, s}[k, \ell]=\delta_{r k} \delta_{s \ell}$ for all $0 \leq k \leq n-1$ and $0 \leq \ell \leq m-1$. Using (iii) of Lemma 2.1.8 we obtain

$$
\begin{aligned}
\underline{\mathfrak{C}}_{\underline{\underline{B}}}^{*} \underline{\mathfrak{C}}_{\underline{B}}\left(\overline{F_{r, s}}\right) & =\underline{\mathfrak{C}}_{\widetilde{\underline{B}}}^{*}\left(\mathfrak{C}_{B_{1}}\left(\overline{F_{r, s}}\right), \ldots, \mathfrak{C}_{B_{P}}\left(\overline{F_{r, s}}\right)\right) \\
& =\underline{\mathfrak{C}_{\underline{B}}^{*}}\left(\sqrt{n m} \mathfrak{\Im}^{-1}\left(\widehat{B}_{1} \odot E_{r, s}\right), \ldots, \sqrt{n m \mathfrak{F}^{-1}}\left(\widehat{B}_{P} \odot E_{r, s}\right)\right) \\
& =\underline{\mathfrak{C}_{\widetilde{B}}^{*}}\left(\sqrt{n m} \mathfrak{\Im}^{-1}\left(\widehat{B}_{1}[r, s] E_{r, s}\right), \ldots, \sqrt{n m \mathfrak{F}^{-1}}\left(\widehat{B}_{P}[r, s] E_{r, s}\right)\right) \\
& =\sqrt{n m} \mathfrak{F}^{-1}\left(\sum_{p=1}^{P} \overline{\widehat{\widetilde{B}}_{p}[r, s]}\left(\widehat{B}_{p}[r, s] E_{r, s}\right)\right)=\left(\sum_{p=1}^{P} \widehat{\widehat{\widetilde{B}}}_{p}[r, s] \widehat{B}_{p}[r, s]\right) \overline{F_{r, s}} .
\end{aligned}
$$

Taking into account that the DFT is a unitary operator, see (i) of Lemma 2.1.8, the above calculation shows that it diagonalizes the operator $\underline{\mathfrak{C}}_{\widetilde{\underline{B}}}^{*} \underline{\mathfrak{C}}_{\underline{B}}$ and its eigenvalues are given by

$$
\left\{\sum_{p=1}^{P}\left(\overline{\widehat{\widetilde{B}}}_{p}[k, \ell] \widehat{B}_{p}[k, \ell]\right): \quad 0 \leq k \leq n-1 \text { and } 0 \leq \ell \leq m-1\right\} .
$$

Let in the following $Y \in \mathbb{R}_{+}^{n \times m}$ be the strong admissibility matrix of $(\underline{B}, \underline{\widetilde{B}})$, see (ii) of 
Definition 3.2.1. We have

$$
\sum_{p=1}^{P} \overline{\widehat{\widetilde{B}}_{p}[k, \ell]} \widehat{B}_{p}[k, \ell]=\widehat{Y}[k, \ell] \sum_{p=1}^{P}\left\|\widehat{B}_{p}[k, \ell]\right\|^{2} \in \mathbb{R}_{+} .
$$

(i) For $\underline{\mathbb{C}}_{\widetilde{B}}^{*} \underline{\mathfrak{C}}_{\underline{B}}$ and all $U \in \mathbb{R}^{n \times m}$ we have by (3.2.1) and by strong admissibility that

$$
\begin{aligned}
& \left(\underline{\mathfrak{C}_{\widetilde{B}}^{*}} \underline{\mathfrak{C}}_{\underline{B}}\right)^{*}(U)=\underline{\mathfrak{C}}_{\underline{B}}^{*} \underline{\mathfrak{C}}_{\widetilde{\underline{B}}}(U) \\
& =\mathfrak{F}^{-1}\left(\sum_{p=1}^{P} \overline{\widehat{B}}_{p}[r, s] \widehat{\widetilde{B}}_{p}[k, \ell] \widehat{U}[k, \ell]\right) \\
& =\mathfrak{F}^{-1}\left(\sum_{p=1}^{P} \overline{(\widehat{Y}[k, \ell])^{-1} \widehat{\widetilde{B}}_{p}[r, s]} \widehat{Y}[k, \ell] \widehat{B}_{p}[k, \ell] \widehat{U}[k, \ell]\right) \\
& =\mathfrak{F}^{-1}\left(\sum_{p=1}^{P} \overline{\widehat{\widetilde{B}}}_{p}[r, s] \widehat{B}_{p}[k, \ell] \widehat{U}[k, \ell]\right)=\underline{\mathfrak{C}}_{\underline{\widetilde{B}}}^{*} \underline{\mathfrak{C}_{\underline{B}}}(U),
\end{aligned}
$$

where the last equality is due to $\widehat{Y}[k, \ell]$ being real for all $0 \leq k \leq n-1$ and $0 \leq$ $\ell \leq m-1$.

Considering $\underline{\mathfrak{C}}_{\underline{B}} \underline{\mathfrak{C}}_{\underline{\underline{B}}}^{*}$ we obtain again via strong admissibility that

$$
\begin{aligned}
\left(\underline{\mathfrak{C}}_{\underline{B}} \underline{\mathfrak{C}_{\underline{B}}^{*}}\right)^{*}(\underline{W}) & =\underline{\mathfrak{C}}_{\underline{\underline{B}}} \underline{\mathfrak{C}}_{\underline{B}}^{*}(\underline{W}) \\
& =\left(\sum_{p=1}^{P}\left(\mathfrak{C}_{\widetilde{B}_{q}} \mathfrak{C}_{B_{p}}^{*}\right)\left(W_{p}\right)\right)_{1 \leq q \leq P} \\
& =\left(\sum_{p=1}^{P} \mathfrak{F}^{-1}\left[\left(\widehat{Y}[k, \ell] \widehat{B}_{q}[k, \ell] \widehat{\widehat{B}}_{p}[k, \ell] \widehat{W}_{p}[k, \ell]\right)_{k=0, \ell=0}^{n-1, m-1}\right]\right)_{1 \leq q \leq P} \\
& =\left(\sum_{p=1}^{P} \mathfrak{F}^{-1}\left[\left(\widehat{B}_{q}[k, \ell] \overline{\widehat{\widetilde{B}}_{p}[k, \ell]} \widehat{W}_{p}[k, \ell]\right)_{k=0, \ell=0}^{n-1, m-1}\right]\right)_{1 \leq q \leq P} \\
& =\left(\sum_{p=1}^{P}\left(\mathfrak{C}_{B_{q}} \mathfrak{C}_{\widetilde{B}_{p}}^{*}\right)\left(W_{p}\right)\right)_{1 \leq q \leq P}=\underline{\mathfrak{C}}_{\underline{B}} \underline{\mathfrak{C}}_{\widetilde{B}}^{*}(\underline{W}),
\end{aligned}
$$

where the third equality uses again that $\widehat{Y}[k, \ell]$ is real for all $0 \leq k \leq n-1$ and $0 \leq$ $\ell \leq m-1$, yielding assertion (i).

(ii) In (3.2.1) we have deduced that the eigenvalues of $\underline{C}_{\widetilde{B}}^{*} \underline{\mathfrak{C}_{B}}$ are given by (3.2.2). By (i) $\underline{\mathfrak{C}}_{\underline{B}} \underline{\mathfrak{C}}_{\underline{\underline{B}}}^{*}$ is self-adjoint and thus diagonalizable by a unitary operator. 
Consider an eigenvalue $v \neq 0$ of $\underline{\mathfrak{C}}_{\vec{B}}^{*} \underline{\mathfrak{C}}_{\underline{B}}$ to an eigenmatrix $U \in \mathbb{C}^{n \times m}$ then we have that $U \notin \operatorname{ker}\left(\underline{\mathfrak{C}}_{\underline{B}}\right)$, hence $\underline{\mathfrak{C}}_{\underline{B}}(U)$ is a family of matrices, not all of which are the 0 -matrix,

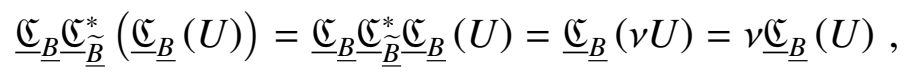

making $v$ an eigenvalue of $\underline{\mathfrak{C}}_{\underline{B}} \underline{\mathfrak{C}}_{\widetilde{B}}^{*}$ to the eigenfamily of matrices $\underline{\mathfrak{C}}_{\underline{B}}(U)$.

Vice versa if $v \neq 0$ is an eigenvalue of $\underline{\mathfrak{C}}_{\underline{B}} \underline{\underline{C}}_{\widetilde{B}}^{*}$ to an eigenfamily of matrices $\underline{W} \in \Gamma$, then in particular $\underline{W} \notin \operatorname{ker}\left(\underline{\mathfrak{C}}_{\underline{\underline{B}}}^{*}\right)$, hence,

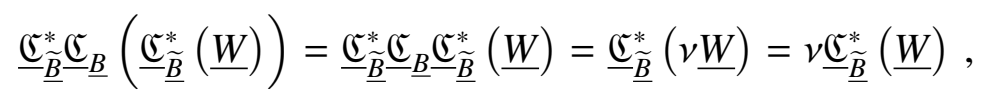

making $v$ an eigenvalue of $\underline{\mathfrak{C}}_{\widetilde{B}}^{*} \underline{\mathfrak{C}}_{\underline{B}}$ to an eigenmatrix $\underline{\mathfrak{C}}_{\widetilde{B}}^{*}(\underline{W})$. In consequence this shows that all non-zero eigenvalues of $\underline{\mathfrak{C}}_{\underline{B}} \underline{\mathfrak{E}}_{\underline{B}}^{*}$ are given in (3.2.2), concluding the proof.

Corollary 3.2.6. Let $(\underline{B}, \underline{\widetilde{B}})$ be strongly admissible. Then, the linear mappings $\underline{\mathbb{C}}_{\widetilde{B}}^{*} \underline{\mathfrak{C}}_{\underline{B}}$ and $\underline{\mathfrak{C}}_{\underline{B}} \underline{\mathfrak{E}}_{\underline{\underline{B}}}^{*}$ are positive semidefinite and have a unique principal square-root, denoted by $\left(\underline{\mathfrak{C}}_{\underline{B}} \underline{\underline{\mathfrak{C}}} \underset{\underline{B}}{*}\right)^{1 / 2}$ and $\left(\underline{\mathfrak{C}}_{\underline{B}}^{*} \underline{\mathfrak{C}}_{\underline{B}}\right)^{1 / 2}$, respectively, where non-zero eigenvalues are given by

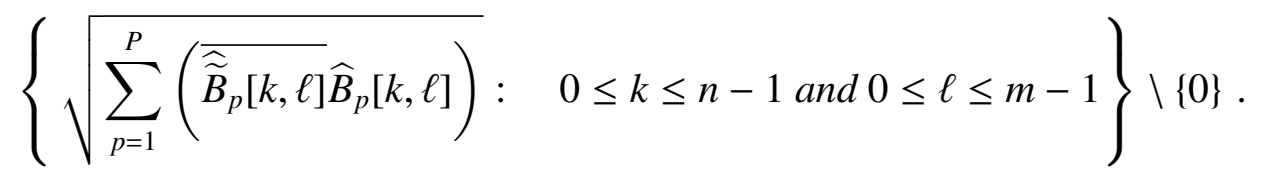

Proof. By strong admissibility and Lemma 3.2.5 the operators $\underline{\mathfrak{C}}_{\underline{B}} \underline{\mathfrak{C}}_{\underline{\underline{B}}}^{*}$ and $\underline{\mathfrak{C}}_{\underline{\underline{B}}}^{*} \underline{\underline{\mathfrak{C}}} \underline{\underline{B}}$ are diagonalizable and their eigenvalues are given by

$$
\sum_{p=1}^{P}\left({\overline{\widetilde{\mathbb{B}_{p}}}[k, \ell] \widehat{B}_{p}}[k, \ell]\right)=Y[k, \ell] \sum_{p=1}^{P}\left\|B_{p}[k, \ell]\right\|^{2} \geq 0,
$$

making $\underline{\mathfrak{C}}_{\underline{B}} \underline{\mathbb{C}}_{\underline{\underline{B}}}^{*}$ and $\underline{\mathfrak{C}}_{\underline{\underline{C}}}^{*} \underline{\mathfrak{C}}_{\underline{B}}$ positive semidefinite.

Remark 3.2.7. Notably the part of Lemma 3.2 .5 concerning the operator $\underline{\mathfrak{C}}_{\underline{\underline{B}}}^{*} \underline{\mathfrak{C}}_{\underline{B}}$ also holds for weak admissibility. However, concerning the operator $\underline{\mathfrak{E}}_{\underline{B}} \mathfrak{C}_{\widetilde{B}}^{*}$ this is not true, since Equation 3.2.3 can not be reproduced. In other words, for weakly admissible $(\underline{B}, \underline{\widetilde{B}})$ the operator $\underline{\mathfrak{C}}_{\underline{B}} \underline{\mathfrak{E}}_{\underline{\underline{B}}}^{*}$ is in general not self-adjoint. 


\subsubsection{The Generalized Algorithm}

Let us state in the following for given input filters $(A, \underline{B}, \underline{\widetilde{B}})$ the generalized Algorithm 3.2.8 and the generalized Problem 3.2.9.

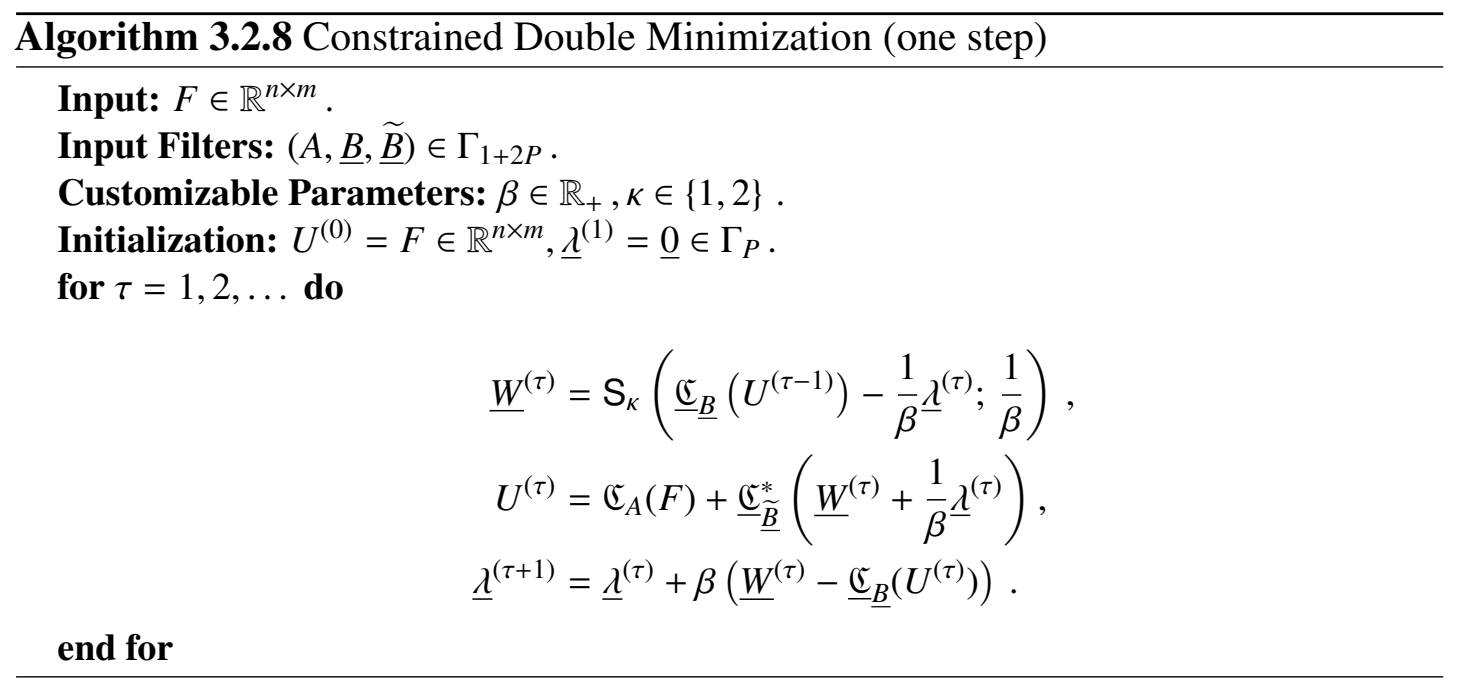

Problem 3.2.9. Given $F \in \mathbb{R}^{n \times m}, \kappa \in\{1,2\}$ and $\beta \in \mathbb{R}_{+}$, as well as, input filters $(A, \underline{B}, \underline{\widetilde{B}}) \in \Gamma_{1+2 P}$. Find a point $\left(U^{\dagger}, \underline{W}^{\dagger}, \underline{\lambda}^{\dagger}\right) \in \Gamma_{1+2 P}$ in the intersection of the following three sets

$$
\begin{aligned}
& \Omega_{1}^{K}:=\left\{(U, \underline{W}, \underline{\lambda}) \in \Gamma_{1+2 P}: \underline{W}=\mathrm{S}_{K}\left(\underline{\mathfrak{C}}_{\underline{B}}(U)-\frac{1}{\beta} \underline{\lambda} ; \frac{1}{\beta}\right)\right\}, \\
& \Omega_{2}^{G}:=\left\{(U, \underline{W}, \underline{\lambda}) \in \Gamma_{1+2 P}: U=\mathfrak{C}_{A}(F)+\underline{\mathfrak{C}}_{\widetilde{B}}^{*}\left(\underline{W}+\frac{1}{\beta} \underline{\lambda}\right)\right\}, \\
& \Omega_{C}:=\left\{(U, \underline{W}, \underline{\lambda}) \in \Gamma_{1+2 P}: \underline{\mathfrak{C}}_{\underline{B}}(U)=\underline{W}\right\} .
\end{aligned}
$$

Let us make an immediate remark about the parameters $\mu, \beta \in \mathbb{R}_{+}$of Problem 3.1.8 and state that Algorithm 3.2.8 solves Problem 3.2.9 and as a special case also Problem 3.1.8. Remark 3.2.10. Note that, while in Problem 3.1.8 the choice of $\beta$ does not alter the solution, cf. Lemma 3.1.4, this is not the case in Problem 3.2.9.

Lemma 3.2.11. Let $F \in \mathbb{R}^{n \times m}, \beta \in \mathbb{R}_{+}, \kappa \in\{1,2\}$ and let $(A, \underline{B}, \underline{\widetilde{B}})$ be input filters. $A$ point $\left(U^{\dagger}, \underline{W^{\dagger}}, \underline{\lambda}^{\dagger}\right) \in \Gamma_{1+2 P}$ is a solution of Problem 3.2.9 if and only if it is a fixed point of Algorithm 3.2.8.

Proof. Follows at once from the respective definitions.

Lemma 3.2.12. Let $F \in \mathbb{R}^{n \times m}, \underline{B} \in \Gamma_{P}, \mu, \beta \in \mathbb{R}_{+}$and $\kappa \in\{1,2\}$. Let $\left(U^{\dagger}, \underline{W}^{\dagger}, \underline{\lambda}^{\dagger}\right) \in$ $\Gamma_{1+2 P}$ be a solution of Problem 3.1.8. Then, there exist input filters $\left(A_{0}, \underline{B}_{0}, \underline{\widetilde{B}}_{0}\right)$ such that $\left(U^{\dagger}, \underline{W}^{\dagger}, \underline{\lambda}^{\dagger}\right)$ is a solution of Problem 3.2.9 defined via $F, \beta, \kappa$ and $\left(A^{0}, \underline{B}^{0}, \underline{\widetilde{B}}^{0}\right)$. 
Proof. Let $F \in \mathbb{R}^{n \times m}, \underline{B} \in \Gamma_{P}, \mu, \beta \in \mathbb{R}_{+}, \kappa \in\{1,2\}$ and $\left(U^{\dagger}, \underline{W}^{\dagger}, \underline{\lambda}^{\dagger}\right)$ be as in the assertion. Define $\underline{B}_{0}=\underline{B}$. Leaving to show for suitable $A^{0}$ and $\underline{\widetilde{B}}^{0}$ that $\left(U^{\dagger}, \underline{W}^{\dagger}, \underline{\lambda}^{\dagger}\right) \in \Omega_{2}^{G}$.

We define

$$
\widehat{A}^{0}[k, \ell]:=\mu\left(\mu+\beta \sum_{p=1}^{P}\left\|\widehat{B}_{p}[k, \ell]\right\|^{2}\right)^{-1},
$$

for $0 \leq k \leq n-1$ and $0 \leq \ell \leq m-1$. Then, $\mathfrak{C}_{A^{0}}=\mu\left(\mu \mathfrak{\mathbb { E }}+\beta \underline{\mathfrak{C}}_{\underline{B}}^{*} \underline{\mathfrak{C}}_{\underline{B}}\right)^{-1}$ and we define

$$
\widehat{\widetilde{B}}_{p}^{0}[k, \ell]:=\beta\left(\mu+\beta \sum_{q=1}^{P}\left\|\widehat{B}_{q}[k, \ell]\right\|^{2}\right)^{-1} \widehat{B}_{p}[k, \ell],
$$

for $0 \leq k \leq n-1$ and $0 \leq \ell \leq m-1$ and $1 \leq p \leq P$. Then, $\widetilde{\mathfrak{C}}_{\widetilde{B}^{0}}^{*}=\beta\left(\mu \mathfrak{E}+\beta \underline{\mathfrak{C}}_{\underline{B}}^{*} \underline{\mathfrak{C}}_{\underline{B}}\right)^{-1} \underline{\mathfrak{C}}_{\underline{B}}^{*}$. Putting the above together we have that $\left(U^{\dagger}, \underline{W^{\dagger}}, \underline{\lambda}^{\dagger}\right) \in \Omega_{2}$ yields $\left(U^{\dagger}, \underline{W}^{\dagger}, \underline{\lambda}^{\dagger}\right) \in \Omega_{2}^{G}$, finishing the proof.

Let us consider a problem formulation in the spirit of Problem 3.1.10A/3.1.10B and Lemma 3.1.11.

Problem 3.2.13A. Given $F \in \mathbb{R}^{n \times m}, \beta \in \mathbb{R}_{+}$and input filters $(A, \underline{B}, \underline{\widetilde{B}}) \in \Gamma_{1+2 P}$, such that $\mathfrak{E}-\underline{\mathbb{C}}_{\widetilde{B}}^{*} \underline{\mathfrak{C}}_{\underline{B}}$ is invertible. Find a point $\left(U^{\dagger}, \underline{\lambda}\right) \in \Gamma_{1+P}$ in the intersection of the following two sets:

$$
\Omega_{S}^{1}:=\left\{(U, \underline{\lambda}) \in \Gamma_{1+P}: \lambda_{p}[k, \ell]\left\{\begin{array}{ll}
=-1 & \text { if } \mathfrak{C}_{B_{p}}(U)[k, \ell]>0 \\
\in[-1,1] & \text { if } \mathfrak{C}_{B_{p}}(U)[k, \ell]=0, \text { for all } k, \ell, p \\
=1 & \text { if } \mathfrak{c}_{B_{p}}(U)[k, \ell]<0
\end{array}\right\},\right.
$$

and

$$
\Omega_{F}^{G}:=\left\{(U, \underline{\lambda}) \in \Gamma_{1+P}: U=\left(\mathfrak{\mathbb { E }}-\underline{\mathbb{C}}_{\widetilde{\underline{B}}}^{*} \underline{\mathfrak{C}}_{\underline{B}}\right)^{-1} \mathfrak{C}_{A}(F)+\frac{1}{\beta}\left(\mathfrak{\mathbb { E }}-\underline{\mathfrak{C}}_{\underline{\underline{\underline{B}}}}^{*} \underline{\mathfrak{C}}_{\underline{B}}\right)^{-1} \underline{\mathfrak{C}}_{\underline{\widetilde{B}}}^{*}(\underline{\lambda})\right\} .
$$

Problem 3.2.13B. Given $F \in \mathbb{R}^{n \times m}, \beta \in \mathbb{R}_{+}$and input filters $(A, \underline{B}, \underline{\widetilde{B}}) \in \Gamma_{1+2 P}$, such that $\mathfrak{E}-\underline{\mathbb{C}}_{\widetilde{B}}^{*} \underline{\mathfrak{C}}_{\underline{B}}$ is invertible. Find a point $\left(U^{\dagger}, \underline{\lambda}\right) \in \Gamma_{1+P}$ in the intersection of the following 
two sets:

$$
\begin{aligned}
\Omega_{S}^{2}:=\{ & (U, \underline{\lambda}) \in \Gamma_{1+P}: \\
& \left(\lambda_{p}[k, \ell]\right)_{p=1}^{P}\left\{\begin{array}{ll}
=-\frac{\left(\mathfrak{C}_{B_{p}}(U)[k, \ell]\right)_{p=1}^{P}}{\left\|\left(\mathfrak{C}_{B_{p}}(U)[k, \ell]\right)_{p=1}^{P}\right\|} & \text { if }\left\|\left(\mathfrak{C}_{B_{p}}(U)[k, \ell]\right)_{p=1}^{P}\right\|>0 \\
\in \mathcal{B}_{1}(0) & \text { if }\left\|\left(\mathfrak{C}_{B_{p}}(U)[k, \ell]\right)_{p=1}^{P}\right\|=0
\end{array}, \text { for all } k, \ell\right\},
\end{aligned}
$$

where $\mathcal{B}_{1}(0)$ is the ball of radius 1 around $0 \in \mathbb{R}^{P}$, and

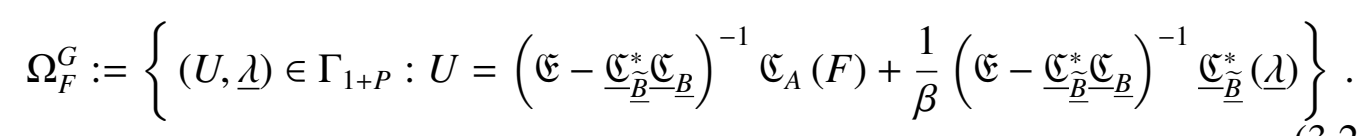

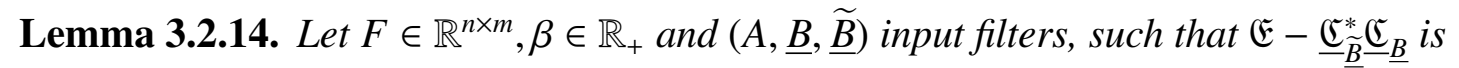
invertible.

(i) For $\kappa=1$ a point $\left(U^{\dagger}, \underline{\lambda}^{\dagger}\right) \in \Gamma_{1+P}$ is a solution of Problem 3.2.13A if and only if there exists $\underline{W}^{\dagger} \in \Gamma_{P}$ such that $\left(U^{\dagger}, \underline{W}^{\dagger}, \underline{\lambda}^{\dagger}\right)$ is a solution of Problem 3.2.9.

(ii) For $\kappa=2$ a point $\left(U^{\dagger}, \underline{\lambda}^{\dagger}\right) \in \Gamma_{1+P}$ is a solution of Problem 3.2.13B if and only if there exists $\underline{W}^{\dagger} \in \Gamma_{P}$ such that $\left(U^{\dagger}, \underline{W}^{\dagger}, \underline{\lambda}^{\dagger}\right)$ is a solution of Problem 3.2.9.

Proof. Analogous to the proof of Lemma 3.1.11

\subsection{Constrained Double Minimization}

To understand Algorithm 3.2.8 further, we relate it its generality to a variational problem. We start by characterizing the first two updating steps of Algorithm 3.2.8 as the minimizers of two functionals. In this way we construct a variational problem that seeks to minimize two functionals simultaneously.

Let in the following $F \in \mathbb{R}^{n \times m}, \kappa \in\{1,2\}, \beta \in \mathbb{R}_{+}$and $(A, \underline{B}, \underline{\widetilde{B}}) \in \Gamma_{1+2 P}$ be input filters. Note, that given $U \in \mathbb{R}^{n \times m}$ and $\underline{\lambda} \in \Gamma_{P}$, the update in $\underline{W} \in \Gamma_{P}$ in Algorithm 3.2.8 is the same as in Algorithm 3.1.5. Hence, by Lemma 3.1.7, we update the $\underline{W}$-component in Algorithm 3.2 .8 by minimizing the functional

$$
\mathcal{J}_{1}(\underline{W}):=|\underline{W}|_{1, K}+\frac{\beta}{2} \| \underline{W}-\left.\underline{\mathfrak{C}}_{\underline{B}}(U)\right|^{2}+\langle\underline{\lambda}, \underline{W}\rangle .
$$

To define the update of $U \in \mathbb{R}^{n \times m}$ in Algorithm 3.2.8 as the solution of a minimization 
problem we first have to construct two operators $\underline{\underline{\mathfrak{C}}}_{\underline{C}}: \Gamma_{P} \rightarrow \Gamma_{P}$ and $\mathfrak{C}_{L}: \mathbb{R}^{n \times m} \rightarrow \mathbb{R}^{n \times m}$.

Construction of $\underline{\underline{C}}_{\underline{C}}$ : Define the auxiliary matrix-family $\underline{G}=\left(G_{p}\right)_{p=1}^{P}$ in the frequency domain by

$$
\widehat{G}_{p}[k, \ell]:= \begin{cases}\left(\overline{\widehat{B}_{p}[k, \ell]}\right)^{-1} \overline{\widehat{\widetilde{B}}_{p}[k, \ell]} & \text { if } \overline{\widehat{B}_{p}[k, \ell]} \neq 0 \\ 1 & \text { else }\end{cases}
$$

for $0 \leq k \leq n-1$ and $0 \leq \ell \leq m-1$. We obtain an entry-wise matrix-family convolution $\underline{\underline{\mathfrak{C}}}_{\underline{G}}: \Gamma_{P} \rightarrow \Gamma_{P}$. Via $\underline{\underline{\mathfrak{C}}}_{\underline{G}}$ we define $\underline{C} \in \Gamma_{P}$ as the matrix-family yielding the entry-wise matrix-family convolution $\underline{\underline{\mathfrak{C}}}_{\underline{C}}=\left(\mathfrak{C}_{C_{p}}\right)_{p=1}^{P}: \Gamma_{P} \rightarrow \Gamma_{P}$ given by

$$
\mathfrak{C}_{C_{p}}:=\left(\mathfrak{E}+\underline{\mathfrak{C}}_{\underline{B}}^{*} \underline{\mathfrak{C}_{B}}\right) \mathfrak{C}_{G_{p}} \quad \text { for } 1 \leq p \leq P .
$$

Remark 3.3.1. Note that if $(\underline{B}, \underline{\widetilde{B}})$ is weakly admissible the above defined $\underline{\underline{\underline{C}}}_{\underline{C}}$ yields via Lemma 2.1.16 the equality

$$
\left(\mathfrak{E}+\underline{\mathfrak{C}}_{\underline{B}}^{*} \underline{\mathfrak{C}}_{\underline{B}}\right)^{-1} \underline{\mathfrak{C}}_{\underline{B}}^{*} \underline{\mathfrak{C}}_{\underline{C}}=\left(\mathfrak{E}+\underline{\mathfrak{C}}_{\underline{B}}^{*} \underline{\mathfrak{C}} \underline{\mathfrak{C}}_{\vec{B}}\right)^{-1}\left(\mathfrak{E}+\underline{\mathfrak{C}}_{\underline{B}}^{*} \underline{\mathfrak{C}} \underline{\mathfrak{C}}_{\vec{B}}\right) \underline{\mathfrak{C}}_{\underline{B}}^{*} \underline{\mathfrak{C}}_{\underline{G}}=\underline{\mathfrak{C}}_{\underline{B}}^{*} \underline{\mathfrak{C}}_{\underline{G}}=\underline{\mathfrak{C}}_{\underline{\widetilde{B}}}^{*},
$$

where the last equality holds true since by weak admissibility we have,

$$
\overline{\widehat{B}_{p}[k, \ell]}=0 \Rightarrow \overline{\widehat{\widetilde{B}}_{p}[k, \ell]}=0, \quad \text { for all } 1 \leq p \leq P, 0 \leq k \leq n-1 \text { and } 0 \leq \ell \leq m-1
$$

Construction of $\mathfrak{C}_{L}$ : Define $L \in \mathbb{R}^{n \times m}$ as the matrix giving rise to the matrix-convolution $\mathfrak{C}_{L}: \mathbb{R}^{n \times m} \rightarrow \mathbb{R}^{n \times m}$ given by

$$
\mathfrak{C}_{L}:=\left(\mathfrak{\mathbb { E }}+\underline{\mathfrak{C}}_{\underline{B}}^{*} \underline{\mathfrak{C}_{B}}\right) \mathfrak{C}_{A}
$$

Remark 3.3.2. For input filters $(A, \underline{B}, \underline{\widetilde{B}})$, we have

$$
\left(\mathfrak{E}+\underline{\mathfrak{C}}_{\underline{B}}^{*} \underline{\mathfrak{C}_{B}}\right)^{-1} \mathfrak{C}_{L}=\mathfrak{C}_{A},
$$

by construction of $\mathfrak{C}_{L}$.

Lemma 3.3.3 below introduces the demanded functional using $\underline{\underline{\mathfrak{C}}}_{\underline{C}}$ and $\mathfrak{C}_{L}$.

Lemma 3.3.3. For given $F \in \mathbb{R}^{n \times m}, \underline{W}, \underline{\lambda} \in \Gamma_{P}, \beta \in \mathbb{R}_{+}$and input filters $(A, \underline{B}, \underline{\widetilde{B}})$, with $(\underline{B}, \underline{\widetilde{B}})$ being weakly admissible. Let $L \in \mathbb{R}^{n \times m}$ and $\underline{C} \in \Gamma_{P}$ be defined via (3.3.5) 
and (3.3.3), respectively. Then, the minimizer of

$$
\mathcal{J}_{2}(U)=\frac{\beta}{2}\left\|\mathfrak{C}_{L}(F)-U\right\|^{2}+\frac{\beta}{2}\left\|\underline{\underline{\mathfrak{C}}}_{\underline{C}}(\underline{W})-\underline{\mathfrak{C}}_{\underline{B}}(U)\right\|^{2}-\left\langle\underline{\underline{\mathfrak{C}}}_{\underline{C}}(\underline{\lambda}), \underline{\mathfrak{C}}_{\underline{B}}(U)\right\rangle,
$$

is given by

$$
U^{\dagger}=\mathfrak{C}_{A}(F)+\underline{\mathfrak{C}}_{\underline{\underline{B}}}^{*}\left(\underline{W}+\frac{1}{\beta} \underline{\lambda}\right) .
$$

Proof. Since $\mathcal{J}_{2}$ is a differentiable, convex function in $U$ a necessary and sufficient condition on a minimizer $U^{\dagger}$ of $\mathcal{J}_{2}$ is given by $\frac{\partial}{\partial U} \mathcal{J}_{2}\left(U^{\dagger}\right)=0$ (see for example Boyd and Vandenberghe [BV04][p.136ff.]). We obtain

$$
\begin{aligned}
& 0=\beta U^{\dagger}-\beta \mathfrak{C}_{L}(F)+\beta\left(\underline{\mathfrak{C}}_{\underline{B}}^{*} \underline{\underline{C}} \underline{\underline{B}}\right)\left(U^{\dagger}\right)-\beta\left(\underline{\mathfrak{C}}_{\underline{B}}^{*} \underline{\mathfrak{C}}_{\underline{C}}\right)(\underline{W})-\left(\underline{\mathfrak{C}}_{\underline{B}}^{*} \underline{\mathfrak{C}}_{\underline{C}}\right)(\underline{\lambda}) \\
& \Rightarrow \quad U^{\dagger}=\left(\mathfrak{E}+\underline{\mathfrak{C}}_{\underline{B}}^{*} \underline{\mathfrak{C}} \underline{\underline{B}}\right)^{-1} \mathfrak{C}_{L}(F)+\left(\mathfrak{E}+\underline{\mathfrak{C}}_{\underline{B}}^{*} \underline{\mathfrak{C}} \underline{\underline{B}}\right)^{-1} \underline{\mathfrak{C}}_{\underline{B}}^{*} \underline{\mathfrak{C}}_{\underline{C}}\left(\underline{W}+\frac{1}{\beta} \underline{\lambda}\right),
\end{aligned}
$$

where by construction $\underline{\mathfrak{C}}_{\underline{B}}^{*} \underline{\mathfrak{C}}_{\underline{B}}$ is positive semi-definite and thus its eigenvalues are given by $\sum_{p=1}^{P} \widehat{B}_{p}[k, \ell] \widehat{B}_{p}[k, \ell] \geq 0$ for all $0 \leq k \leq n-1$ and $0 \leq \ell \leq m-1$, yielding that by Lemma 2.1.8 the inverses of $\left(\mathfrak{E}+\underline{\mathfrak{C}}_{\underline{B}}^{*} \underline{\mathfrak{C}}_{\underline{B}}\right)$ exists. By Remarks 3.3.1 and 3.3.2 we have

$$
U^{\dagger}=\mathfrak{C}_{A}(F)+\underline{\mathfrak{C}}_{\underline{\underline{B}}}^{*}\left(\underline{W}+\frac{1}{\beta} \underline{\lambda}\right)
$$

In fact, one could say Algorithm 3.2.8 is still of an ADMM-type. Its iteration step is the alternating minimization of two functionals. However, we no longer minimizer the same functional in both updating steps as in Algorithm 3.1.5. One of the two functionals is changed such that given $\underline{\underline{\underline{E}}}_{\underline{C}} \neq \underline{\underline{\mathfrak{E}}}$ the two functionals $\mathcal{J}_{1}$ and $\mathcal{J}_{2}$ differ on the common terms of $\underline{W}$ and $U$. Hence, the saddle-point structure of Problem 3.1.3 can not be recovered this way. Instead our best formulation of a variational problem solved by Algorithm 3.2.8 to this end is given by Problem 3.3.4 below, motivating the name constrained double minimization.

Problem 3.3.4 (Constrained Double Minimization). For a given $F \in \mathbb{R}^{n \times m}, \kappa \in\{1,2\}$ and $\beta \in \mathbb{R}_{+}$let $\mathcal{J}_{1}$ and $\mathcal{J}_{2}$ be defined as in (3.3.1) and (3.3.6), respectively. Then, in case of existence, find an element $\left(U^{\dagger}, \underline{W^{\dagger}}, \underline{\lambda^{\dagger}}\right) \in \Gamma_{1+2 P}$ such that the following two minimization problems are solved simultaneously,

$$
\underline{W}^{\dagger}=\operatorname{argmin}_{\underline{W} \in \Gamma_{P}} \mathcal{J}_{1}\left(U^{\dagger}, \underline{W}, \underline{\lambda^{\dagger}}\right) \text {, as well as }, U^{\dagger}=\operatorname{argmin}_{U \in \mathbb{R}^{n \times m}} \mathcal{J}_{2}\left(U, \underline{W^{\dagger}}, \underline{\lambda}^{\dagger}\right) \text {, }
$$


under the constraint

$$
\underline{\underline{C}}_{\underline{B}}\left(U^{\dagger}\right)=\underline{W}^{\dagger} .
$$

Let us prove the equivalence of Problem 3.3.4 and Problem 3.2.9.

Lemma 3.3.5. Let $F \in \mathbb{R}^{n \times m}, \kappa \in\{1,2\}, \beta \in \mathbb{R}_{+}$and let $(A, \underline{B}, \underline{\widetilde{B}})$ be input filters such that $(\underline{B}, \underline{\widetilde{B}})$ are weakly admissible and satisfy the $(N E P C)$. A point $\left(U^{\dagger}, \underline{W}^{\dagger}, \underline{\lambda}^{\dagger}\right)$ is a solution of Problem 3.2.9 if and only if it is a solution of Problem 3.3.4.

Proof. Let $\left(U^{\dagger}, \underline{W}^{\dagger}, \underline{\lambda}^{\dagger}\right)$ be a solution of Problem 3.2.9, then by $\left(U^{\dagger}, \underline{W^{\dagger}}, \underline{\lambda}^{\dagger}\right) \in \Omega_{C}$ we have

$$
\underline{\mathfrak{c}}_{\underline{B}}\left(U^{\dagger}\right)=\underline{W}^{\dagger} .
$$

Furthermore, by (i) of Lemma 3.1.7 and Lemma 3.3.3 we obtain

$$
\underline{W}^{\dagger}=\operatorname{argmin}_{\underline{W} \in \Gamma_{P}} \mathcal{J}_{1}\left(U^{\dagger}, \underline{W}, \underline{\lambda^{\dagger}}\right) \text {, as well as, } U^{\dagger}=\operatorname{argmin}_{U \in \mathbb{R}^{n \times m}} \mathcal{J}_{2}\left(U, \underline{W^{\dagger}}, \underline{\lambda}^{\dagger}\right) \text {, }
$$

hence, $\left(U^{\dagger}, \underline{W}^{\dagger}, \underline{\lambda}^{\dagger}\right)$ is a solution of Problem 3.3.4.

Conversely, let $\left(U^{\dagger}, \underline{W^{\dagger}}, \underline{\lambda}^{\dagger}\right)$ be a solution to Problem 3.3.4, then it follows immediately that $\left(U^{\dagger}, \underline{W}^{\dagger}, \underline{\lambda}^{\dagger}\right) \in \Omega_{C}$ by the constraint. Furthermore, we can compute again via (i) of Lemma 3.1.7 and Lemma 3.3.3 that $\left(U^{\dagger}, \underline{W}^{\dagger}, \underline{\lambda}^{\dagger}\right) \in \Omega_{1}^{\kappa} \cap \Omega_{2}^{G}$. Yielding that $\left(U^{\dagger}, \underline{W}^{\dagger}, \underline{\lambda}^{\dagger}\right)$ is a solution of Problem 3.2.9.

Remark 3.3.6. A consequence of the unavailability of a convex minimization problem solved by Algorithm 3.2.8 is that the existence of a fixed point of Algorithm 3.2.8 can no longer be derived this way. Hence, to show convergence of Algorithm 3.2.8 in general, we first need to show under which circumstances a solution to Problem 3.3.4 (or equivalently 3.2.9) exists. 


\section{CHAPTER 4}

\section{Convergence Analysis}

In the following chapter we deal with the question of convergence guarantees for Algorithm 3.2.8. We use the characterizations of admissibility and the (NEPC), (SNEPC) and (CPC) criteria, cf. Definition 3.2.1, to show under which conditions we can guarantee the existence and uniqueness of a fixed point of Algorithm 3.2.8 and its convergences under the assumption that a fixed point exists. For an overview see Table 4.1.

\subsection{Existence of a Fixed Point}

As discussed in Remark 3.3.6, the existence of a fixed point for Algorithm 3.2.8 can not be derived at once as in the classical $\ell^{1}$-regularization. Generalizing to Problem 3.3.4 we can not retrieve the convex minimization problem structure of Problem 3.1.2 that yields the existence of a unique fixed point. Instead a possible fixed point is characterized by a double minimization of two convex functionals. While each single minimization problem is strictly convex and thus has a unique solution, the existence of a simultaneous minimizer is not clear.

In this chapter we provide existence of a fixed point under the condition that $(\underline{B}, \underline{\widetilde{B}})$ are weakly admissible and satisfy the (CPC). Moreover, we give a counterexample in which no fixed point exists given input filters that satisfy the (NEPC). If $(\underline{B}, \underline{\widetilde{B}})$ satisfy

Table 4.1

Overview of the presented proofs

\begin{tabular}{c||c|c|c|c|c}
\hline $\begin{array}{c}\text { Admissibility } \\
\text { Eigenvalue condition }\end{array}$ & $\begin{array}{c}\text { strong } \\
\text { (CPC) }\end{array}$ & $\begin{array}{c}\text { weak } \\
\text { (CPC) }\end{array}$ & $\begin{array}{c}\text { strong } \\
\text { (SNEPC) }\end{array}$ & $\begin{array}{c}\text { weak } \\
\text { (SNEPC) }\end{array}$ & $\begin{array}{c}\text { strong/weak } \\
\text { (NEPC) }\end{array}$ \\
\hline \hline Existence & $\checkmark$ & $\checkmark$ & $?$ & $?$ & $\times$ \\
Convergence given Existence & $\checkmark$ & $?$ & $\checkmark$ & $?$ & - \\
Convexity of fixed point set & $\checkmark$ & $?$ & $\checkmark$ & $?$ & - \\
Uniqueness & $\checkmark$ & $?$ & $?$ & $?$ & -
\end{tabular}


the (SNEPC) we neither can show the existence of a fixed point, nor can we find a counterexample, at this point.

Let us give an overview over the existence proof for the case that $(\underline{B}, \underline{\widetilde{B}})$ are weakly admissible and satisfy the (CPC). By Lemma 3.2.14 we can consider the equivalent Problems 3.2.13A/3.2.13B - depending on the choice of $\kappa \in\{1,2\}$ - of finding a point in the intersection of the affine, linear subspace

$$
\Omega_{F}^{G}:=\left\{(U, \underline{\lambda}) \in \Gamma_{1+P}: U=\left(\mathfrak{\mathbb { E }}-\underline{\mathfrak{C}}_{\underline{\underline{B}}}^{*} \underline{\mathfrak{C}_{B}}\right)^{-1} \mathfrak{C}_{A}(F)+\frac{1}{\beta}\left(\mathfrak{\mathbb { E }}-\underline{\mathfrak{C}}_{\underline{\underline{\underline{C}}}}^{*} \underline{\mathbb{C}}\right)^{-1} \underline{\mathfrak{C}}_{\underline{\underline{B}}}^{*}(\underline{\lambda})\right\} .
$$

and the non-linear set

$$
\Omega_{S}^{1}:=\left\{(U, \underline{\lambda}) \in \Gamma_{1+P}: \lambda_{p}[k, \ell]\left\{\begin{array}{ll}
=-1 & \text { if } \mathfrak{C}_{B_{p}}(U)[k, \ell]>0 \\
\in[-1,1] & \text { if } \mathfrak{C}_{B_{p}}(U)[k, \ell]=0, \text { for all } k, \ell, p \\
=1 & \text { if } \mathfrak{C}_{B_{p}}(U)[k, \ell]<0
\end{array}\right\},\right.
$$

in the case $\kappa=1$ or the non-linear set

$$
\begin{aligned}
\Omega_{S}^{2}:=\{ & (U, \underline{\lambda}) \in \Gamma_{1+P}: \\
& \left(\lambda_{p}[k, \ell]\right)_{p=1}^{P}\left\{\begin{array}{ll}
=-\frac{\left(\mathfrak{C}_{B_{p}}(U)[k, \ell]\right)_{p=1}^{P}}{\left\|\left(\mathfrak{C}_{B_{p}}(U)[k, \ell]\right)_{p=1}^{P}\right\|} & \text { if }\left\|\left(\mathfrak{C}_{B_{p}}(U)[k, \ell]\right)_{p=1}^{P}\right\|>0 \\
\in \mathcal{B}_{1}(0) & \text { if }\left\|\left(\mathfrak{C}_{B_{p}}(U)[k, \ell]\right)_{p=1}^{P}\right\|=0
\end{array}, \text { for all } k, \ell\right\},
\end{aligned}
$$

in the case of $\kappa=2$.

To show that the intersection of $\Omega_{F}^{G}$ and $\Omega_{S}^{\kappa}$ is non-empty for both $\kappa$ we argue as follows.

(i) First, in Chapter 4.1.1 we show via Proposition 4.1.1 that there exists a point in the intersection of an affine, linear subspace $\Xi$ and a non-linear set $\widetilde{\Omega}$, under certain assumptions.

(ii) Second, in Chapter 4.1.2 we show that we can apply this Proposition 4.1 .1 to $\Omega_{F}^{G}$ and an approximation of $\Omega_{S}^{\kappa}$ for both $\kappa \in\{1,2\}$.

(iii) Finally, in Chapter 4.1.3 we conclude that a fixed point of Algorithm 3.2.8 exists in the case that $(\underline{B}, \underline{\widetilde{B}})$ are weakly admissible and satisfy the (CPC).

In Chapter 4.1.4 we consider the case of relaxing the (CPC) to the (NEPC). As mentioned we give a counterexample in the case that $(\underline{B}, \underline{\widetilde{B}})$ do not satisfy the (SNEPC). 
Furthermore, we give some intuition why the above described roadmap will not work for the more general case of $(\underline{B}, \underline{\widetilde{B}})$ being weakly admissible and satisfying the (SNEPC).

\subsubsection{The Intersection Proposition}

The following Proposition 4.1.1 is tailored for its later use on $\Omega_{F}^{G}$ and $\Omega_{S}^{\kappa}$. We make use of the definitions made in Chapter 2.3 regarding mapping degree theory.

Proposition 4.1.1. Let $\Xi, \Omega \subset \mathbb{R}^{n}$ be two affine, linear, non-trivial subspaces that intersect at exactly one point $\Xi \cap \Omega=\{v\}$ and that jointly span the whole space, i.e. $\Xi \oplus \Omega=\mathbb{R}^{n}$. Furthermore, let $\theta_{1}$ be the first principal angle between $\Xi$ and $\Omega$ and let

$$
\phi: \Omega \rightarrow \mathbb{R}^{n}
$$

be a continuous map such that there exists a constant $C>0$ satisfying

$$
\|\phi(\omega)-\omega\| \leq C, \text { for all } \omega \in \Omega .
$$

Then,

(i) $\Xi \cap \phi(\Omega)$ is non-empty.

(ii) Moreover, we have that

$$
\|u-v\| \leq \widetilde{C}:=C\left(1+\frac{1}{\sin \left(\theta_{1}\right)}\right),
$$

for all $u \in \Xi \cap \phi(\Omega)$.

\section{Proof. Reduction to linear subspaces:}

Let $\Xi, \Omega, v$ and $\phi: \Omega \rightarrow \mathbb{R}^{N}$ as well as $C>0$ be as in the assertion. Then defining

$$
\begin{aligned}
& \bar{\Xi}=\left\{\xi-v \in \mathbb{R}^{n}: \xi \in \Xi\right\}, \quad \bar{\Omega}=\left\{\omega-v \in \mathbb{R}^{n}: \omega \in \Omega\right\}, \\
& \bar{\phi}: \bar{\Omega} \rightarrow \mathbb{R}^{N} ; \bar{\omega} \mapsto \phi(\bar{\omega}+v)-v,
\end{aligned}
$$

also satisfy the prerequisites: $\bar{\Xi}$ and $\bar{\Omega}$ are now linear subspaces of $\mathbb{R}^{n}$ and have as their unique intersection point 0 , while $\bar{\phi}$ is continuous and $\|\bar{\phi}(\bar{\omega})-\bar{\omega}\| \leq C$ for all $\bar{\omega} \in \bar{\Omega}$. Lastly, note that by definition the first principal angle between $\Xi$ and $\Omega$ coincides with the first principal angle between $\bar{\Xi}$ and $\bar{\Omega}$.

If now $\bar{\Xi} \cap \bar{\phi}(\bar{\Omega})$ is non-empty and $d(\bar{\Xi} \cap \bar{\phi}(\bar{\Omega}),\{0\}) \leq \widetilde{C}$, then via adding $\{v\}$ we obtain that also $\Xi \cap \phi(\Omega)$ is non-empty and $d(\Xi \cap \phi(\Omega),\{v\}) \leq \widetilde{C}$. Hence, without loss of 
generality we can consider $\Xi$ and $\Omega$ to be linear subspaces.

(i) Existence: Let in the following $\operatorname{dim}(\Xi)=n-m$ and accordingly $\operatorname{dim}(\Omega)=m$. We can choose $m$ linearly independent, pairwise orthogonal vectors $h_{1}, \ldots, h_{m}$ of unit length orthogonal to $\Xi$, i.e.

$$
\left\langle h_{j}, \xi\right\rangle=0 \text { for all } \xi \in \Xi \text { and } 1 \leq j \leq m \text {. }
$$

Let now $H:=\left(h_{1}, \ldots, h_{m}\right) \in \mathbb{R}^{N \times J}$ be the matrix composed of these vectors, then we can define the mappings

$$
\begin{aligned}
& \Psi_{1}: \Omega \rightarrow \mathbb{R}^{m} ; \omega \mapsto H^{T} \omega \\
& \Psi_{2}: \Omega \rightarrow \mathbb{R}^{m} ; \omega \mapsto H^{T} \phi(\omega) .
\end{aligned}
$$

Let furthermore, $h_{m+1}, \ldots, h_{n}$ be an orthonormal basis of $\Xi$, then $h_{1}, h_{2}, \ldots, h_{n}$ is an orthonormal basis of $\mathbb{R}^{n}$ and we can define the unitary, square matrix $\widetilde{H}=$ $\left(h_{1}, \ldots, h_{n}\right)$ as well as the matrix $S=\left(h_{m+1}, \ldots, h_{n}\right)$. We have for any $\omega \in \Omega$ by orthonormality of the $h_{j}$ 's that

$$
\left\|\Psi_{1}(\omega)\right\|^{2}=\left\|H^{T} \omega\right\|^{2}=\left\|H^{T} \omega\right\|^{2}+\left\|S^{T} \omega\right\|^{2}-\left\|S^{T} \omega\right\|^{2}=\|\omega\|^{2}-\left\|S^{T} \omega\right\|^{2},
$$

For the second term we obtain

$$
\begin{aligned}
\left\|S^{T} \omega\right\|^{2} & =\sum_{j=m+1}^{n}\left(h_{j}^{T} \omega\right)^{2} \leq \max _{\xi \in \Xi,\|\xi\|=1}\left(\xi^{T} \omega\right)^{2} \\
& \leq\|\omega\|^{2} \quad \max _{\substack{\xi \in \Xi:\|\xi\|=1 \\
\widetilde{\omega} \in \Omega:\|\widetilde{\omega}\|=1}}\left(\xi^{T} \widetilde{\omega}\right)^{2} \\
& =\|\omega\|^{2} \cos ^{2}\left(\theta_{1}\right),
\end{aligned}
$$

where the first inequality stems from the fact that we can chose $h_{m+1}$ as $\left\|\mathcal{P}_{\Xi}(\omega)\right\|^{-1} \mathcal{P}_{\Xi}(\omega)$, where $\mathcal{P}_{\Xi}(\omega)$ is the orthogonal projection of $\omega$ onto the linear subspace $\Xi$. Hence, by (4.1.4) for all $\omega \in \Omega$ we have

$$
\left\|\Psi_{1}(\omega)\right\|^{2}=\|\omega\|^{2}-\left\|S^{T} \omega\right\|^{2} \geq\|\omega\|^{2}\left(1-\cos ^{2}\left(\theta_{1}\right)\right)=\|\omega\|^{2} \sin ^{2}\left(\theta_{1}\right) .
$$

Since $\theta_{1} \in(0, \pi / 2]$ we obtain

$$
\left\|\Psi_{1}(\omega)\right\| \geq\|\omega\| \sin \left(\theta_{1}\right)
$$


Let now

$$
\mathcal{B}:=\mathcal{B}_{C\left(1+2 / \sin \left(\theta_{1}\right)\right)}(0)=\left\{\omega \in \Omega:\|\omega\| \leq C\left(1+\frac{2}{\sin \left(\theta_{1}\right)}\right)\right\}
$$

and denote its boundary within $\Omega$ by $\stackrel{\circ}{\mathcal{B}}$. For a point on the boundary $a \in \stackrel{\circ}{\mathcal{B}}$ we compute by (4.1.6) that

$$
\left\|\Psi_{1}(a)\right\| \geq\|a\| \sin \left(\theta_{1}\right)=C\left(\sin \left(\theta_{1}\right)+2\right) \geq 2 C .
$$

On the other hand, by hypothesis on $\phi$ we have for all $a \in \Omega$, in particular for all $a \in \stackrel{\circ}{\mathcal{B}}$, also

$$
\begin{aligned}
\left\|\Psi_{2}(a)-\Psi_{1}(a)\right\| & =\left\|H^{T} \phi(a)-H^{T} a\right\|=\left\|H^{T}(\phi(a)-a)\right\| \\
& \leq\|\phi(a)-a\| \leq C,
\end{aligned}
$$

again by orthonormality of the columns of $H$.

The homotopy given by

$$
v:[0,1] \times \Omega \rightarrow \mathbb{R}^{m}, \quad(t, w) \mapsto t \Psi_{1}(w)+(1-t) \Psi_{2}(w),
$$

between $\Psi_{1}$ and $\Psi_{2}$, thus satisfies by (4.1.7) and (4.1.8) for all $a \in \stackrel{\circ}{\text { the relation }}$

$$
\begin{aligned}
\|v(t, a)\| & =\left\|t \Psi_{1}(a)+(1-t) \Psi_{2}(a)\right\| \\
& =\left\|\Psi_{1}(a)-(1-t)\left(\Psi_{1}(a)-\Psi_{2}(a)\right)\right\| \\
& \geq\left\|\Psi_{1}(a)\right\|-\left\|(1-t)\left(\Psi_{1}(a)-\Psi_{2}(a)\right)\right\| \geq C,
\end{aligned}
$$

for all $t \in[0,1]$. Hence, we obtain

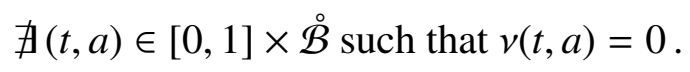

Last, choose an orthonormal basis $b_{1}, b_{2}, \ldots, b_{m}$ of the linear subspace $\Omega$ and define the isomorphism

$$
f: \mathbb{R}^{m} \rightarrow \Omega, \quad\left(x_{1}, x_{2}, \ldots, x_{m}\right)^{T} \mapsto \sum_{j=1}^{m} x_{j} b_{j}
$$

We have that

$$
v_{f}:[0,1] \times f^{-1}(B), \quad(t, x) \mapsto v(t, f(x)),
$$


is a homotopy between $\Psi_{1} \circ f$ and $\Psi_{2} \circ f$ and in particular by (4.1.9) we have

$$
\nexists(t, a) \in[0,1] \times\left(f^{-1}(\stackrel{\circ}{)})\right) \text { such that } v_{f}(t, a)=0 .
$$

Hence, we can apply Lemma 2.3.3 to obtain

$$
\begin{aligned}
\operatorname{deg}\left(\Psi_{2} \circ f, f^{-1}(\mathcal{B}), 0\right) & =\operatorname{deg}\left(\Psi_{1} \circ f, f^{-1}(\mathcal{B}), 0\right) \\
& =\operatorname{sign}\left(\operatorname{det}\left(D_{0}\left[\Phi_{1} \circ f\right]\right)\right) \\
& =\operatorname{sign}\left(\operatorname{det}\left(D_{0}\left[\Phi_{1}\right] D_{0}[f]\right)\right) \\
& =\operatorname{sign}\left(\operatorname{det}\left(H^{T}\left(b_{1}, \ldots, b_{m}\right)\right)\right) \neq 0,
\end{aligned}
$$

because $\operatorname{ker}\left(H^{T}\left(b_{1}, \ldots, b_{m}\right)\right)=\{0\}$. By Corollary 2.3.4 we obtain the existence of at least one $x \in \mathbb{R}^{m}$ such that $H^{T} \circ \phi \circ f(x)=0$, yielding that there must exist at least one $\omega \in \phi(\Omega)$ such that $H^{T} \omega=0$. By construction of $H$ such a $\omega$ is in $\Xi$ proving the first assertion.

(ii) Inequality: For the second assertion let $\omega^{\dagger} \in \Xi \cap \phi(\Omega)$ and $\omega \in \Omega$, then if $\phi(\omega)=\omega^{\dagger}$ we have by assertion on $\phi$ that $\left\|\omega^{\dagger}-\omega\right\| \leq C$ and by (4.1.8) that

$$
\left\|\Psi_{1}(\omega)\right\|=\left\|H^{T} \omega\right\|=\left\|H^{T} \omega-H^{T} \omega^{\dagger}\right\|=\left\|\Psi_{1}(\omega)-\Psi_{2}(\omega)\right\| \leq C
$$

Furthermore, by (4.1.6) we have

$$
\|\omega\| \leq \frac{C}{\sin \left(\theta_{1}\right)}
$$

yielding finally,

$$
\left\|\omega^{\dagger}-0\right\| \leq\left\|\omega^{\dagger}-\omega\right\|+\|\omega\| \leq C\left(1+\frac{1}{\sin \left(\theta_{1}\right)}\right)
$$

\subsubsection{Preparations and Set-Valued Calculus}

Now we construct a linear subspace $\Omega_{S}^{0}$ that is close to the non-linear sets $\Omega_{S}^{\kappa}$, for $\kappa=1,2$, as well as a continuous mappings $\phi^{\kappa}$ mapping from $\Omega_{S}^{0}$ to an approximation of $\Omega_{S}^{\kappa}$, for $\kappa=1,2$, satisfying the distance relation required. For this purpose consider

$$
\Omega_{S}^{0}:=\left\{(U, \underline{\lambda}) \in \Gamma_{1+P}: \underline{\lambda}=\underline{0}\right\} .
$$

We start by showing that $\Omega_{S}^{0}$ and $\Omega_{F}^{G}$ satisfy the assumptions on $\Omega$ and $\Xi$ in Proposi- 
tion 4.1.1.

Lemma 4.1.2. Let $F \in \mathbb{R}^{n \times m}, \beta \in \mathbb{R}_{+}$and $(A, \underline{B}, \underline{\widetilde{B}})$ be input filters, with $(\underline{B}, \underline{\widetilde{B}})$ weakly admissible and satisfying the (CPC). Let $\Omega_{S}^{0}$ and $\Omega_{F}^{G}$ be defined as in (4.1.10) and (4.1.1), respectively. Then,

(i) $\Omega_{S}^{0}$ and $\Omega_{F}^{G}$ are affine linear subspaces.

(ii) $\Omega_{S}^{0}$ and $\Omega_{F}^{G}$ intersect in a unique point.

(iii) $\Omega_{S}^{0} \oplus \Omega_{F}^{G}=\Gamma_{1+P}$.

Proof. (i) Follows by construction.

(ii) By using the (CPC) we obtain

$$
\left(\left(\mathfrak{E}-\underline{\mathfrak{C}}_{\underline{\underline{B}}}^{*} \underline{\mathfrak{C}}_{\underline{B}}\right)^{-1} \mathfrak{C}_{A}(F), \underline{0}\right) \in \Omega_{S}^{0} \cap \Omega_{F}^{G},
$$

hence, the intersection is non-empty. Now suppose $\left(U_{1}, \underline{\lambda}_{1}\right),\left(U_{2}, \underline{\lambda}_{2}\right) \in \Omega_{S}^{0} \cap \Omega_{F}^{G}$, then we have

$$
\underline{\lambda}_{t}=\underline{0} \text { for } t=1,2, \quad \Rightarrow \quad\left(\underline{\lambda}_{1}-\underline{\lambda}_{2}\right)=\underline{0}
$$

yielding,

$$
U_{1}-U_{2}=\frac{1}{\beta}\left(\mathfrak{\mathbb { E }}-\underline{\mathfrak{C}}_{\underline{\underline{B}}}^{*} \underline{\underline{C}} \underline{\underline{B}}\right)^{-1} \underline{\mathfrak{C}_{\overparen{\underline{B}}}^{*}}\left(\underline{\lambda}_{1}-\underline{\lambda}_{2}\right)=0 .
$$

Hence, $\left(U_{1}, \underline{\lambda}_{1}\right)=\left(U_{2}, \underline{\lambda}_{2}\right)$, i.e. the intersection point is unique, finishing (ii).

(iii) The (affine) dimension of $\Omega_{S}^{0}$ is $n m$, while that of $\Omega_{F}^{G}$ is $P n m$, since it is the graph of a linear function from $\Gamma_{P}$ to $\mathbb{R}^{n \times m}$. By (ii) it follows $\operatorname{dim}\left(\Omega_{S}^{0} \cap \Omega_{F}^{G}\right)=0$, hence, by dimension formula

$$
\Omega_{S}^{0} \oplus \Omega_{F}^{G}=\Gamma_{1+P}
$$

To construct suitable $\phi^{k}$ we need approximations of the set-valued functions given below, see Chapter 2.4 for basic definitions in set-valued function calculus. Consider for a given $\underline{B} \in \Gamma_{P}$,

$\mathrm{Y}^{1}: \mathbb{R}^{n \times m} \rightrightarrows \Gamma_{P}$,

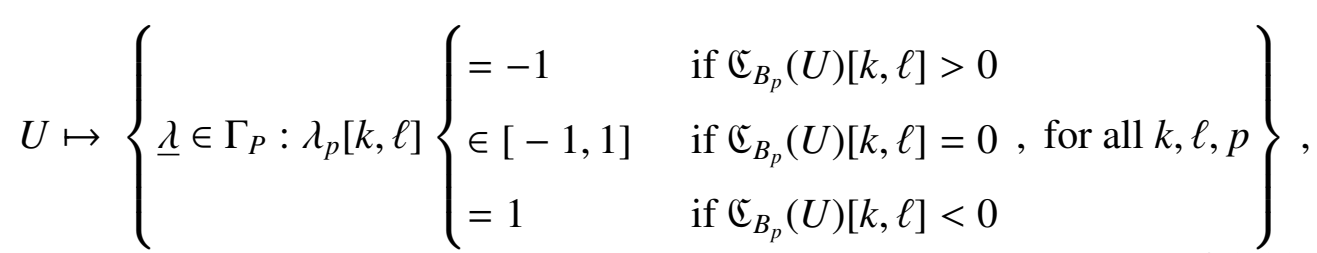




$$
\begin{aligned}
& \text { for } \kappa=1 \text { and } \\
& Y^{2}: \mathbb{R}^{n \times m} \rightrightarrows \Gamma_{P}, \\
& U \mapsto\left\{\underline{\lambda} \in \Gamma_{P}:\right. \\
& \left(\lambda_{p}[k, \ell]\right)_{p=1}^{P}\left\{\begin{array}{ll}
=-\frac{\left(\mathfrak{C}_{B_{p}}(U)[k, \ell]\right)_{p=1}^{P}}{\left\|\left(\mathfrak{C}_{B_{p}}(U)[k, \ell]\right]_{p=1}^{P}\right\|} & \text { if }\left\|\left(\mathfrak{C}_{B_{p}}(U)[k, \ell]\right)_{p=1}^{P}\right\|>0 \\
\in \mathcal{B}_{1}(0) & \text { if }\left\|\left(\mathfrak{C}_{B_{p}}(U)[k, \ell]\right)_{p=1}^{P}\right\|=0
\end{array}, \text { for all } k, \ell\right\},
\end{aligned}
$$

for $\kappa=2$. We show that the graph of $Y^{\kappa}$ is given by $\Omega_{S}^{\kappa}$, for $\kappa=1,2$, respectively. Since, we need a single-valued function to apply Proposition 4.1.1, we will use a known result to approximate $\Upsilon^{1}$ and $\Upsilon^{2}$. We start by proving useful properties for $Y^{1}$ and $\Upsilon^{2}$.

Lemma 4.1.3. Let $\kappa \in\{1,2\}$ and $\underline{B} \in \Gamma_{P}$. Then, the set-valued mapping $Y^{\kappa}$ defined in (4.1.11) and (4.1.12), respectively, satisfies the following.

(i) $\operatorname{Graph}\left(\mathrm{Y}^{\kappa}\right)$, see Definition 2.4.1, is equal to $\Omega_{S}^{\kappa}$, defined for $\kappa=1$ in (4.1.2) and for $\kappa=2$ in (4.1.3).

(ii) $\mathrm{Y}^{\kappa}$ is convex-valued.

Proof. (i) Given $(U, \underline{\lambda}) \in \Omega_{S}^{1}$ then by definition

$$
\lambda_{p}[k, \ell] \begin{cases}=-1 & \text { if } \mathfrak{C}_{B_{p}}(U)[k, \ell]>0 \\ \in[-1,1] & \text { if } \mathfrak{C}_{B_{p}}(U)[k, \ell]=0, \text { for all } k, \ell, p, \\ =1 & \text { if } \mathfrak{c}_{B_{p}}(U)[k, \ell]<0\end{cases}
$$

which is equivalent with $\underline{\lambda} \in \mathrm{Y}^{1}(U)$, i.e. $(U, \underline{\lambda}) \in \operatorname{Graph}\left(\mathrm{Y}^{1}\right)$.

Given $(U, \underline{\lambda}) \in \Omega_{S}^{2}$ then by definition

$$
\left(\lambda_{p}[k, \ell]\right)_{p=1}^{P}\left\{\begin{array}{ll}
=-\frac{\left(\mathfrak{C}_{B_{p}}(U)[k, \ell]\right]_{p=1}^{P}}{\left\|\left(\mathfrak{C}_{B_{p}}(U)[k, \ell]\right)_{p=1}^{P}\right\|} & \text { if }\left\|\left(\mathfrak{C}_{B_{p}}(U)[k, \ell]\right)_{p=1}^{P}\right\|>0 \\
\in \mathcal{B}_{1}(0) & \text { if }\left\|\left(\mathfrak{C}_{B_{p}}(U)[k, \ell]\right)_{p=1}^{P}\right\|=0
\end{array}, \text { for all } k, \ell\right.
$$

which is equivalent with $\underline{\lambda} \in \mathrm{Y}^{2}(U)$, i.e. $(U, \underline{\lambda}) \in \operatorname{Graph}\left(\mathrm{Y}^{2}\right)$.

(ii) Let $U \in \mathbb{R}^{n \times m}$ and $\underline{\lambda}^{1}, \underline{\lambda}^{2} \in \mathrm{Y}^{1}(U)$. Then 
- If $\mathfrak{C}_{B_{p}}(U)[k, \ell] \neq 0$ we have

$$
\begin{aligned}
\lambda_{p}^{1}[k, \ell] & =\lambda_{p}^{2}[k, \ell] \\
\Rightarrow t \lambda_{p}^{1}[k, \ell]+(1-t) \lambda_{p}^{2}[k, \ell] & =\left\{\begin{array}{ll}
=-1 & \text { if } \mathfrak{C}_{B_{p}}(U)[k, \ell]>0 \\
=1 & \text { if } \mathfrak{C}_{B_{p}}(U)[k, \ell]<0
\end{array},\right.
\end{aligned}
$$

for all $t \in[0,1]$.

- If $\mathfrak{C}_{B_{p}}(U)[k, \ell]=0$ we have

$$
\lambda_{p}^{1}[k, \ell], \lambda_{p}^{2}[k, \ell] \in[-1,1] \quad \Rightarrow \quad \lambda_{p}^{1}[k, \ell]+(1-t) \lambda_{p}^{2}[k, \ell] \in[-1,1],
$$

for all $t \in[0,1]$.

Let $U \in \mathbb{R}^{n \times m}$ and $\underline{\lambda}^{1}, \underline{\lambda}^{2} \in \mathrm{Y}^{2}(U)$. Then

- If $\left\|\left(\mathfrak{C}_{B_{p}}(U)[k, \ell]\right)_{p=1}^{P}\right\|>0$ we have

$$
\begin{aligned}
\left(\lambda_{p}^{1}[k, \ell]\right)_{p=1}^{P} & =\left(\lambda_{p}^{2}[k, \ell]\right)_{p=1}^{P} \\
\Rightarrow t\left(\lambda_{p}^{1}[k, \ell]\right)_{p=1}^{P}+(1-t)\left(\lambda_{p}^{2}[k, \ell]\right)_{p=1}^{P} & =\frac{\left(\mathfrak{C}_{B_{p}}(U)[k, \ell]\right)_{p=1}^{P}}{\left\|\left(\mathfrak{C}_{B_{p}}(U)[k, \ell]\right)_{p=1}^{P}\right\|} .
\end{aligned}
$$

- If $\left\|\left(\mathfrak{C}_{B_{p}}(U)[k, \ell]\right)_{p=1}^{P}\right\|=0$ we have

$$
\begin{gathered}
\left(\lambda_{p}^{1}[k, \ell]\right)_{p=1}^{P},\left(\lambda_{p}^{2}[k, \ell]\right)_{p=1}^{P} \in \mathcal{B}_{1}(0) \\
\Rightarrow \quad\left(\lambda_{p}^{1}[k, \ell]\right)_{p=1}^{P}+(1-t)\left(\lambda_{p}^{2}[k, \ell]\right)_{p=1}^{P} \in \mathcal{B}_{1}(0) \text { for all } t \in[0,1] .
\end{gathered}
$$

Lemma 4.1.4. Let $\kappa \in\{1,2\}$ and $\underline{B} \in \Gamma_{P}$. Then, the set-valued mapping $Y^{\kappa}$ defined in (4.1.11) and (4.1.12), respectively, are upper semi-continuous, see Definition 2.4.2.

\section{Proof. (i) The case of $\mathrm{Y}^{1}$.}

Let $\epsilon>0$ and $U_{0} \in \mathbb{R}^{n \times m}$ and let in the following

$$
\mathcal{Z}^{1}:=\left\{(k, \ell, p): \widetilde{\mathfrak{C}}_{B_{p}}\left(U_{0}\right)[k, \ell]=0\right\},
$$

be the set of indices for which $\mathfrak{C}_{B_{p}}\left(U_{0}\right)[k, \ell]$ is 0 .

Then we can define

$$
\rho_{U_{0}}^{1}:=\min _{(k, \ell, p) \notin \mathcal{Z}^{1}}\left|\mathfrak{C}_{B_{p}}\left(U_{0}\right)[k, \ell]\right|>0 .
$$


Further, note that the mappings

$$
V \mapsto \mathfrak{C}_{B_{p}}(V)[k, \ell]
$$

are continuous by construction of $\underline{\mathfrak{C}}_{\underline{B}}$. Hence, given $(k, \ell, p) \notin \mathcal{Z}^{1}$ we can find $\delta_{(k, \ell, p), \rho_{U_{0}}^{1}}^{1}>0$ such that for all $U \in \mathbb{R}^{n \times m}$ with $\left\|U_{0}-U\right\|_{2}<\delta_{(k, \ell, p), \rho_{U_{0}}^{1}}^{1}$ we have

$$
\left|\mathfrak{C}_{B_{p}}\left(U_{0}\right)[k, \ell]-\mathfrak{C}_{B_{p}}(U)[k, \ell]\right|<\frac{\rho_{U_{0}}^{1}}{2} .
$$

Now, define

$$
\delta_{\rho_{U_{0}}^{1}}^{1}:=\min _{(k, \ell, p) \notin \mathcal{Z}^{1}} \delta_{(k, \ell, p), \rho_{U_{0}}^{1}}^{1} .
$$

Let now $U \in \mathbb{R}^{n \times m}$ with $\left\|U_{0}-U\right\|<\delta_{\rho_{U_{0}}^{1}}^{1}$, then for any $(k, \ell, p) \notin \mathcal{Z}^{1}$ we have

$$
\left|\mathfrak{C}_{B_{p}}\left(U_{0}\right)[k, \ell]-\mathfrak{C}_{B_{p}}(U)[k, \ell]\right|<\frac{\rho_{U_{0}}^{1}}{2} \Rightarrow \mathfrak{C}_{B_{p}}\left(U_{0}\right)[k, \ell] \mathfrak{C}_{B_{p}}(U)[k, \ell]=1,
$$

hence, for any $\underline{\lambda}^{0} \in \mathrm{Y}^{1}\left(U_{0}\right)$ and $\underline{\lambda} \in \mathrm{Y}^{1}(U)$ we have

$$
\lambda_{p}^{0}[k, \ell]=\lambda_{p}[k, \ell]
$$

Let now $(k, \ell, p) \in \mathcal{Z}^{1}$. Then, given any $\underline{\lambda} \in \mathrm{Y}^{1}(U)$ we have

$$
\lambda_{p}[k, \ell] \in[-1,1],
$$

since $\mathfrak{C}_{B_{p}}\left(U_{0}\right)[k, \ell]=0$ by construction of $\mathrm{Y}^{1}$ we can choose $\underline{\lambda}^{0} \in \mathrm{Y}^{1}\left(U_{0}\right)$ such that

$$
\lambda_{p}^{0}[k, \ell]=\lambda_{p}[k, \ell]
$$

Both cases together yield

$$
\min _{Z_{0} \in Y^{1}\left(U_{0}\right)}\left\|Z_{0}-Z\right\|=0<\epsilon \text { for all } Z \in Y^{1}(U) .
$$

(ii) The case of $Y^{2}$.

Let $\epsilon>0$ and $U_{0} \in \mathbb{R}^{n \times m}$. We define first via

$$
\mathcal{Z}^{2}:=\left\{(k, \ell):\left\|\left(\mathfrak{C}_{B_{p}}\left(U_{0}\right)[k, \ell]\right)_{p=1}^{P}\right\|=0\right\},
$$

the set of indices for which $\left\|\left(\mathfrak{C}_{B_{p}}\left(U_{0}\right)[k, \ell]\right)_{p=1}^{P}\right\|$ is 0 . 
Then we can define

$$
\rho_{U_{0}}^{2}:=\min _{(k, \ell) \notin \mathcal{Z}^{2}}\left\|\left(\mathfrak{C}_{B_{p}}\left(U_{0}\right)[k, \ell]\right)_{p=1}^{P}\right\|>0
$$

Further, note that the maps

$$
V \mapsto\left(\mathfrak{C}_{B_{p}}(V)[k, \ell]\right)_{p=1}^{P},
$$

are continuous by construction by construction of $\underline{\mathfrak{C}}_{\underline{B}}$. Hence, for $(k, \ell) \notin \mathcal{Z}^{2}$ we can choose $\delta_{(k, \ell), \rho_{U_{0}}^{2}}^{2}>0$ such that for all $U \in \mathbb{R}^{n \times m}$ with $\left\|U_{0}-U\right\|<\delta_{(k, \ell), \rho_{U_{0}}^{2}}^{2}$ we have

$$
\left\|\left(\mathfrak{C}_{B_{p}}\left(U_{0}\right)[k, \ell]\right)_{p=1}^{P}-\left(\mathfrak{C}_{B_{p}}(U)[k, \ell]\right)_{p=1}^{P}\right\|<\frac{\rho_{U_{0}}^{2}}{2} .
$$

So for all $(k, \ell) \notin \mathcal{Z}^{2}$ the continuous mapping

$$
V \mapsto \frac{\left(\mathfrak{C}_{B_{p}}(V)[k, \ell]\right)_{p=1}^{P}}{\left\|\left(\mathfrak{C}_{B_{p}}(V)[k, \ell]\right)_{p=1}^{P}\right\|},
$$

is well defined on the ball $\mathcal{B}_{\delta_{(k, \ell), \rho^{2}\left(U_{0}\right)}}\left(\left(\mathfrak{C}_{B_{p}}\left(U_{0}\right)[k, \ell]\right)_{p=1}^{P}\right)$. Let $0<\widetilde{\delta}_{(k, \ell), \rho_{U_{0}}^{2}}^{2}<\delta_{(k, \ell), \rho_{U_{0}}^{2}}^{2}$ be such that for all $U \in \mathbb{R}^{n \times m}$ with $\left\|U_{0}-U\right\|<\widetilde{\delta}_{(k, \ell), \rho_{U_{0}}^{2}}^{2}$ we have

$$
\left\|\frac{\left(\mathfrak{C}_{B_{p}}\left(U_{0}\right)[k, \ell]\right)_{p=1}^{P}}{\left\|\left(\mathfrak{C}_{B_{p}}\left(U_{0}\right)[k, \ell]\right)_{p=1}^{P}\right\|}-\frac{\left(\mathfrak{C}_{B_{p}}(U)[k, \ell]\right)_{p=1}^{P}}{\left\|\left(\mathfrak{C}_{B_{p}}(U)[k, \ell]\right)_{p=1}^{P}\right\|}\right\|<\frac{\epsilon}{2 m n}, .
$$

Now, define

$$
\delta_{\rho_{U_{0}}^{2}}^{2}:=\min _{(k, \ell) \notin \mathcal{Z}^{2}} \widetilde{\delta}_{(k, \ell), \rho_{U_{0}}^{2}}^{2}
$$

Let now $U \in \mathbb{R}^{n \times m}$ with $\left\|U_{0}-U\right\|<\delta_{\rho_{U_{0}}^{2}}^{2}$, then for any $(k, \ell) \notin \mathcal{Z}^{2}$ Equation (4.1.15) holds. Hence, for any $\underline{\lambda}^{0} \in \mathrm{Y}^{2}\left(U_{0}\right)$ and $\underline{\lambda} \in \mathrm{Y}^{2}(U)$ we have

$$
\left\|\left(\lambda_{p}^{0}[k, \ell]\right)_{p=1}^{P}-\left(\lambda_{p}[k, \ell]\right)_{p=1}^{P}\right\|<\frac{\epsilon}{2 m n} .
$$

Let now $(k, \ell) \in \mathcal{Z}^{2}$. Then, given any $\underline{\lambda} \in \mathrm{Y}^{2}(U)$ we have

$$
\left(\lambda_{p}[k, \ell]\right)_{p=1}^{P} \in \mathcal{B}_{1}(0)
$$

since $\left\|\left(\mathfrak{C}_{B_{p}}\left(U_{0}\right)[k, \ell]\right)_{p=1}^{P}\right\|=0$ by construction of $\mathrm{Y}^{2}$ we can choose $\underline{\lambda}^{0} \in \mathrm{Y}^{2}\left(U_{0}\right)$ 
such that

$$
\left(\lambda_{p}^{0}[k, \ell]\right)_{p=1}^{P}=\left(\lambda_{p}[k, \ell]\right)_{p=1}^{P} .
$$

Both cases together yield

$$
\min _{Z_{0} \in Y^{2}\left(U_{0}\right)}\left\|Z_{0}-Z\right\|<\epsilon \text { for all } Z \in Y^{2}(U) .
$$

The following Theorem, shown by Cellina [Cel69][p.19,The.1], linking upper semicontinuity of set-valued functions to a continuous single-valued approximation will be needed, for the proof we refer to the original paper.

Theorem 4.1.5 ([Cel69][Theorem 1]). Let $\Omega \subset \mathbb{R}^{n}$ be compact and $\Upsilon: \Omega \rightrightarrows \mathbb{R}^{m}$ be an upper semicontinuous set-valued mapping with convex values. For $\epsilon>0$ arbitrary, there exists a continuous single valued mapping

$$
v: \Omega \rightarrow\left\{y \in \mathbb{R}^{m}: \exists \widetilde{y} \in \Upsilon(\Omega) \text { such that }\|y-\tilde{y}\|<\epsilon\right\} \cap \operatorname{co}(\Upsilon(\Omega)),
$$

where $\operatorname{co}(\Upsilon(\Omega))$ denotes the convex hull of $\Upsilon(\Omega)$, such that we have

$$
\sup _{x \in \operatorname{Graph}(v)}\left(\inf _{y \in \operatorname{Graph}(\Upsilon)}(\|x-y\|)\right) \leq \epsilon .
$$

Now we can infer the later needed Corollary.

Corollary 4.1.6. Let $\kappa \in\{1,2\}$ and $\underline{B} \in \Gamma_{P}$. Let $Y^{\kappa}: \mathbb{R}^{n \times m} \rightrightarrows \Gamma_{P}$ be defined as in (4.1.11), or (4.1.12), respectively. For $U_{0} \in \mathbb{R}^{n \times m}$ and $R>0$ arbitrary define the ball

$$
\mathcal{B}:=\mathcal{B}_{R}\left(U_{0}\right):=\left\{U \in \mathbb{R}^{n \times m}:\left\|U-U_{0}\right\| \leq R\right\}
$$

Then, for every $\epsilon>0$ there exist continuous functions

$$
v_{\epsilon, R}^{\kappa}: \mathcal{B} \rightarrow \Gamma_{P}, \text { for } \kappa=1,2,
$$

such that

$$
\sup _{x \in \operatorname{Graph}\left(v_{\epsilon, R}^{\kappa}\right)}\left(\inf _{y \in \operatorname{Graph}\left(\left.\Upsilon^{\kappa}\right|_{\mathcal{B}}\right)}(\|x-y\|)\right) \leq \epsilon,
$$

where $\left.\mathrm{Y}^{\kappa}\right|_{\mathcal{B}}$ is the restriction of $\mathrm{Y}^{\kappa}$ to the ball $\mathcal{B}$, for $\kappa=1,2$.

Proof. Let us check the prerequisites of Theorem 4.1.5: $\mathcal{B}$ is a compact subset of the Euclidean space $\mathbb{R}^{n \times m}$, the set valued mapping $\left.\mathrm{Y}^{\kappa}\right|_{\mathcal{B}}$ is by (ii) of Lemma 4.1 .4 u.s.c. and 
it is by Lemma 4.1.3 convex-valued for $\kappa=1,2$. Hence, by Theorem 4.1.5 for all $\epsilon>0$ there exists continuous mappings $v_{\epsilon, R}^{\kappa}: \mathcal{B} \rightarrow \Gamma_{P}$ such that

$$
\sup _{x \in \operatorname{Graph}\left(v_{\epsilon, R}^{\kappa}\right)}\left(\inf _{y \in \operatorname{Graph}\left(\Upsilon^{\kappa} \mid \mathcal{B}\right)}(\|x-y\|)\right) \leq \epsilon,
$$

for $\kappa=1,2$.

\subsubsection{Existence of a Fixed Point}

After having collected the prerequisites needed we are now able to state and prove the main theorem of this chapter.

Theorem 4.1.7. Let $F \in \mathbb{R}^{n \times m}, \kappa \in\{1,2\}, \beta \in \mathbb{R}_{+}$and let $(A, \underline{B}, \underline{\widetilde{B}}) \in \Gamma_{1+2 P}$ be input filters, with $(\underline{B}, \underline{\widetilde{B}})$ weakly admissible and satisfying the $(C P C)$. Given the sets $\Omega_{F}^{G}$ and $\Omega_{S}^{K}$ as defined in (4.1.1) and (4.1.2) or (4.1.3), respectively, their intersection is non-empty.

Proof. Let us start the proof by picking up the constructions made in 4.1.2:

- Let the space $\Omega_{S}^{0}$ be the linear subspace defined in (4.1.10). Furthermore, let $\left(U_{0}, \underline{\lambda}_{0}\right) \in \Omega_{S}^{0} \cap \Omega_{F}^{G}$ be the unique intersection point of $\Omega_{S}^{0}$ and $\Omega_{F}^{G}$, that exists by Lemma 4.1.2. Lastly, denote by $\theta_{1}$ the first principal angle of $\Omega_{S}^{0}$ and $\Omega_{F}^{G}$, see Defition 2.3.5, which is by Lemma 4.1.2 an element in the interval $\left(0, \frac{\pi}{2}\right]$.

- We have constructed the set-valued mappings $Y^{\kappa}: \mathbb{R}^{n \times m} \rightarrow \Gamma_{P}$ in (4.1.11) and (4.1.12), for $\kappa=1,2$, whose graph is given by $\Omega_{S}^{\kappa}$, respectively, see Lemma 4.1.3.

- For each ball $\mathcal{B} \subset \mathbb{R}^{n \times m}, \kappa \in\{1,2\}$ of radius $R>0$ around $U_{0}, \kappa \in\{1,2\}$ and each $\epsilon>0$ there exists a continuous function $v_{\epsilon, R}^{\kappa}: \mathcal{B} \rightarrow \Gamma_{P}$ such that

$$
\sup _{x \in \operatorname{Graph}\left(v_{\epsilon, R}^{\kappa}\right)}\left(\inf _{y \in \operatorname{Graph}\left(\left.\Upsilon^{\kappa}\right|_{\mathcal{B}}\right)}(\|x-y\|)\right) \leq \epsilon,
$$

First, we define for $R_{0}:=(P n m+2)\left(1+1 / \sin ^{2}\left(\theta_{1}\right)\right)$ the ball

$$
\mathcal{B}_{0}:=\left\{U \in \mathbb{R}^{n \times m}:\left\|U-U_{0}\right\| \leq R_{0}=(P n m+2)\left(1+\frac{1}{\sin \left(\theta_{1}\right)}\right)\right\},
$$

around $U_{0}$. Let $\epsilon_{r}=\frac{1}{r}$ with $r \in \mathbb{N}$ be a sequence converging to 0 for $r \rightarrow \infty$, then we denote for $\kappa \in\{1,2\}$ in the following the single-valued continuous function $v_{\epsilon_{r}, R}^{\kappa}$ given 
by Corollary 4.1 .6 for $\epsilon_{r}=\frac{1}{r}$ and $R_{0}$ simply by $v_{r}^{\kappa}$.

Now define for $\kappa \in\{1,2\}$ mappings $\phi_{r}^{\kappa}: \Omega_{S}^{0} \rightarrow \Gamma_{1+P}$ by

$$
\phi_{r}^{\kappa}(U, \underline{0})=\left(U, v_{r}^{K}\left(\mathcal{P}_{\mathcal{B}_{0}}(U)\right)\right)
$$

where $\mathcal{P}_{\mathcal{B}_{0}}(U)$ is the orthogonal projection of $U$ onto the convex set $\mathcal{B}_{0}$. We have by definition of $v_{r}^{\kappa}$ and $\mathcal{P}_{\mathcal{B}_{0}}$ that $\phi_{r}^{\kappa}$ is a continuous map. Furthermore, we have by definition of $Y^{\kappa}$ for all $U \in \mathbb{R}^{n \times m}$ and each $\underline{\lambda} \in Y^{\kappa}(U)$ that

$$
\|\underline{\lambda}\| \leq P n m
$$

holds, for $\kappa=1,2$.

By construction of $v_{r}^{\kappa}$ we have,

$$
\sup _{x \in \operatorname{Graph}\left(v_{r}^{k}\right)}\left(\inf _{y \in \operatorname{Graph}\left(\left.\Upsilon^{\kappa}\right|_{\mathcal{B}_{0}}\right)}(\|x-y\|)\right) \leq \frac{1}{r}
$$

we can deduce that for all $U \in \mathcal{B}_{0}$ there exists a $\underline{\lambda}^{U} \in \mathrm{Y}^{\kappa}(U)$ with

$$
\left\|v_{r}^{K}(U)-\underline{\lambda}^{U}\right\| \leq \frac{1}{r}
$$

This yields for all $U \in \Omega_{S}^{0}$ that

$$
\left\|(U, \underline{0})-\phi_{r}^{K}(U, \underline{0})\right\| \leq P n m+1
$$

for all $r \in \mathbb{N}$ and $\kappa \in\{1,2\}$.

By the above constructed $\phi_{r}^{\kappa}: \Omega_{S}^{0} \rightarrow \Gamma_{1+P}$ and Lemma 4.1.2 we have that for all $\kappa \in$ $\{1,2\}$ the sets $\Omega_{S}^{0}, \Omega_{F}^{G}$ and the mapping $\phi_{r}^{\kappa}$ satisfy the prerequisites of Proposition 4.1.1 for all $r \in \mathbb{N}$. Fix in the following $\kappa \in\{1,2\}$. Then, there exists at least one point in the intersection of $\phi_{r}^{\kappa}\left(\Omega_{S}^{0}\right)$ and $\Omega_{F}^{G}$. Choose a sequence $\left\{\left(U_{r}^{\dagger}, \underline{\lambda}_{r}^{\dagger}\right)\right\}_{r \in \mathbb{N}}$ such that

$$
\left(U_{r}^{\dagger}, \underline{\lambda}_{r}^{\dagger}\right) \in \Omega_{F}^{G} \cap \phi_{r}^{\kappa}\left(\Omega_{S}^{0}\right) \text { for } r \in \mathbb{N}
$$

Using 4.1.18 we can use (ii) of Proposition 4.1.1 to additionally obtain,

$$
\left\|\left(U_{r}^{\dagger}, \underline{\lambda}_{r}^{\dagger}\right)-\left(U_{0}, \underline{\lambda}_{0}\right)\right\| \leq(P n m+1)\left(1+\frac{1}{\sin \left(\theta_{1}\right)}\right)
$$

for the previously defined unique point $\left(U_{0}, \underline{\lambda}_{0}\right) \in \Omega_{F}^{G} \cap \Omega_{S}^{0}$. 
In particular, we have

$$
\left\|U_{r}^{\dagger}-U_{0}\right\| \leq(P n m+1)\left(1+\frac{1}{\sin \left(\theta_{1}\right)}\right)
$$

hence, $U_{r}^{\dagger} \in \mathcal{B}_{0}$ and thus by construction

$$
\underline{\lambda}_{r}^{\dagger}=v_{r}^{\kappa}\left(U_{r}^{\dagger}\right)
$$

for all $r \in \mathbb{N}$. Since the $\left(U_{r}^{\dagger}, \underline{\lambda}_{r}^{\dagger}\right)$ lie by (4.1.19) in a compact ball $K$ around $\left(U_{0}, \underline{\lambda}_{0}\right)$ there exists a convergent subsequence

$$
\left(U_{r_{t}}^{\dagger}, \underline{\lambda}_{t}^{\dagger}\right) \stackrel{t \rightarrow \infty}{\longrightarrow}\left(U^{\dagger}, \underline{\lambda}^{\dagger}\right)
$$

for some $\left(U^{\dagger}, \underline{\lambda}^{\dagger}\right) \in K$.

Since, $\left(U_{r_{t}}^{\dagger}, \underline{\lambda}_{r_{t}}^{\dagger}\right) \in \Omega_{F}$ for all $t \in \mathbb{N}$ and $\Omega_{F}$ is closed we deduce

$$
\left(U^{\dagger}, \underline{\lambda}^{\dagger}\right) \in \Omega_{F}
$$

On the other hand we have for all $t \in \mathbb{N}$ by (4.1.20) and (4.1.18) that

$$
\inf _{y \in \operatorname{Graph}\left(\Upsilon^{\kappa}\right)}\left(\left\|\left(U_{r_{t}}^{\dagger}, \underline{\lambda}_{t}^{\dagger}\right)-y\right\|\right) \leq \frac{1}{r},
$$

hence, by Lemma 4.1.3,

$$
\inf _{y \in \Omega_{S}^{k}}\left(\left\|\left(U_{r_{t}}^{\dagger}, \underline{\lambda}_{r_{t}}^{\dagger}\right)-y\right\|\right) \leq \frac{1}{r}
$$

yielding $\left(U^{\dagger}, \underline{\lambda}^{\dagger}\right) \in \Omega_{S}^{K}$, since $\frac{1}{r_{t}} \stackrel{t \rightarrow \infty}{\longrightarrow} 0$ and $\Omega_{S}^{K}$ is closed, finishing the proof.

\subsubsection{Relaxation of the (CPC)}

In this chapter the question of relaxing the (CPC) condition in Theorem 4.1 .7 is discussed.

Let us start by giving a counter example in the case that $(\underline{B}, \underline{\widetilde{B}})$ satisfies the (NEPC), but not the (SNEPC), see Definition 3.2.1.

Example 4.1.8. Let in the following $m=n=2, \kappa=1$ and $\beta=1$, we choose $P=1$ and 
define the input filters

$$
A:=\left(\begin{array}{ll}
1 & 0 \\
0 & 0
\end{array}\right) \quad B=\widetilde{B}:=\frac{1}{4}\left(\begin{array}{cc}
2 & -1 \\
-1 & 0
\end{array}\right) .
$$

By definition of $B=\widetilde{B}$ we have that the above defined input filters $(B, \widetilde{B})$ are strongly admissible. Furthermore, we can compute,

$$
\widehat{B}=\widehat{\widetilde{B}}=\frac{1}{2}\left(\begin{array}{ll}
0 & 1 \\
1 & 2
\end{array}\right)
$$

yielding,

$$
\overline{\widehat{B}} \odot \widehat{B}=\frac{1}{4}\left(\begin{array}{ll}
0 & 1 \\
1 & 4
\end{array}\right),
$$

hence the (NEPC) is satisfied. However, by

$$
\widehat{A}=\left(\begin{array}{ll}
1 & 1 \\
1 & 1
\end{array}\right)
$$

we have that $(A, \underline{B}, \underline{\widetilde{B}})$ does not satisfy the (SNEPC).

Let $F \in \mathbb{R}^{n \times m}, \beta \in \mathbb{R}_{+}$arbitrary, and let $\kappa \in\{1,2\}, \beta \in \mathbb{R}_{+}$and input filters $(A, \underline{B}, \underline{\widetilde{B}})$ be given as above, assume $\left(U^{\dagger}, \underline{W}^{\dagger}, \underline{\lambda}^{\dagger}\right) \in \Gamma_{1+2 P}$ is a solution of Problem 3.2.9. Then, by $\left(U^{\dagger}, \underline{W}^{\dagger}, \underline{\lambda}^{\dagger}\right) \in \Omega_{C}$ we have $\mathfrak{C}_{B}\left(U^{\dagger}\right)=\underline{W}^{\dagger}$. Moreover, using also $\left(U^{\dagger}, \underline{W^{\dagger}}, \underline{\lambda}^{\dagger}\right) \in \Omega_{2}^{G}$ we obtain

$$
U^{\dagger}=F+\mathfrak{C}_{B}^{*}\left(\mathfrak{C}_{B}\left(U^{\dagger}\right)+\underline{\lambda}^{\dagger}\right) .
$$

Let us denote in the following

$$
\widehat{U}=\left(\begin{array}{ll}
\hat{u}_{00} & \hat{u}_{01} \\
\hat{u}_{10} & \hat{u}_{11}
\end{array}\right), \quad \widehat{F}=\left(\begin{array}{cc}
\hat{f}_{00} & \hat{f}_{01} \\
\hat{f}_{10} & \hat{f}_{11}
\end{array}\right), \quad \widehat{\lambda}=\left(\begin{array}{ll}
\hat{\lambda}_{00} & \hat{\lambda}_{01} \\
\hat{\lambda}_{10} & \hat{\lambda}_{11}
\end{array}\right) .
$$

Then (4.1.21) becomes,

$$
\begin{aligned}
\left(\begin{array}{cc}
\hat{u}_{00} & \hat{u}_{01} \\
\hat{u}_{10} & \hat{u}_{11}
\end{array}\right) & =\left(\begin{array}{cc}
\hat{f}_{00} & \hat{f}_{01} \\
\hat{f}_{10} & \hat{f}_{11}
\end{array}\right)+\frac{1}{2}\left(\begin{array}{ll}
0 & 1 \\
1 & 2
\end{array}\right) \odot\left(\frac{1}{2}\left(\begin{array}{cc}
0 & \hat{u}_{01} \\
\hat{u}_{10} & 2 \hat{u}_{11}
\end{array}\right)+\left(\begin{array}{ll}
\hat{\lambda}_{00} & \hat{\lambda}_{01} \\
\hat{\lambda}_{10} & \hat{\lambda}_{11}
\end{array}\right)\right) \\
\Rightarrow \quad \frac{1}{4}\left(\begin{array}{cc}
4 \hat{u}_{00} & 3 \hat{u}_{01} \\
3 \hat{u}_{10} & 0
\end{array}\right) & =\left(\begin{array}{ll}
\hat{f}_{00} & \hat{f}_{01} \\
\hat{f}_{10} & \hat{f}_{11}
\end{array}\right)+\frac{1}{2}\left(\begin{array}{cc}
0 & \hat{\lambda}_{01} \\
\hat{\lambda}_{10} & 2 \hat{\lambda}_{11}
\end{array}\right) .
\end{aligned}
$$


Hence, we have

$$
\hat{f}_{11}=-\hat{\lambda}_{11}
$$

but, by $\left(U^{\dagger}, \underline{W}^{\dagger}, \underline{\lambda}^{\dagger}\right) \in \Omega_{1}^{1} \cap \Omega_{C}$ we obtain $\hat{\lambda}_{11} \in[-4,4]$, due to $\lambda[k, \ell] \in[-1,1]$ for all $0 \leq k, \ell \leq 1$. Hence, choosing $F$ such that $\left|\hat{f}_{11}\right|>4$ we end up with a contradiction.

It is clear that in the above Example 4.1 .8 we rely on $(A, \underline{B}, \underline{\widetilde{B}})$ not satisfying the (SNEPC) to obtain a contradiction to the fixed point statement. However, given that $(A, \underline{B}, \underline{\widetilde{B}})$ satisfy the (SNEPC) and that $(\underline{B}, \underline{\widetilde{B}})$ are weakly admissible, the existence of a fixed point of Algorithm 3.2.8 is an open question. Let us give an idea why our reasoning from Chapters 4.1 .1 to 4.1 .3 fails in the case that the input filters $(A, \underline{B}, \underline{\widetilde{B}})$ satisfy the (SNEPC), but $(\underline{B}, \underline{\widetilde{B}})$ do not satisfy the (CPC).

We construct a problem formulation that is similar to Problem 3.2.13A/3.2.13B, which is valid for input filters $(A, \underline{B}, \underline{\widetilde{B}})$ that satisfy the (SNEPC). For this purpose let us define the set

$$
\mathcal{Z}:=\left\{(k, \ell) \in\{0, \ldots, n-1\} \times\{1, \ldots, m-1\}: \sum_{p=1}^{P} \overline{\widehat{\widetilde{B}}}_{p}[k, \ell] \widehat{B}_{p}[k, \ell]=1\right\},
$$

of all indices $(k, \ell)$ for which the (CPC) does not hold. For simplicity of later calculations, define the following matrix $T \in \mathbb{R}^{n \times m}$ in the frequency domain:

$$
\widehat{T}[k, \ell]:= \begin{cases}\left(1-\sum_{p=1}^{P} \overline{\widehat{\widetilde{B}}_{p}[k, \ell]} \widehat{B}[k, \ell]\right)^{-1} & \text { if }(k, \ell) \notin \mathcal{Z} \\ 0 & \text { if }(k, \ell) \in \mathcal{Z}\end{cases}
$$

We find the following equivalent characterization of Problem 3.2.9.

Lemma 4.1.9. Let $F \in \mathbb{R}^{n \times m}, \kappa \in\{1,2\}, \beta \in \mathbb{R}_{+}$and let $(\underline{B}, \underline{\widetilde{B}}) \in \Gamma_{1+2 P}$ be input filters that satisfy the $(\operatorname{SNEPC})$, with $(\underline{B}, \underline{\widetilde{B}})$ being weakly admissible. Define $T \in \mathbb{R}^{n \times m}$ via (4.2.18). Then, a point $\left(U^{\dagger}, \underline{\lambda}^{\dagger}\right) \in \Gamma_{1+P}$ is an intersection point of the following two sets

$$
\begin{aligned}
& \Omega_{S+}^{1}:=\left\{(U, \underline{\lambda}) \in \Gamma_{1+P}: \lambda_{p}[k, \ell] \begin{cases}=-1 & \text { if } \mathfrak{C}_{B_{p}}(U)[k, \ell]>0 \\
\in[-1,1] & \text { if } \mathfrak{C}_{B_{p}}(U)[k, \ell]=0, \text { for all } k, \ell, p, \\
=1 & \text { if } \mathfrak{C}_{B_{p}}(U)[k, \ell]<0\end{cases} \right. \\
& \text { and } \left.\sum_{p=1}^{P} \overline{\widehat{\widetilde{B}}}_{p}[k, \ell] \widehat{\lambda}_{p}[k, \ell]=0, \text { for all }(k, \ell) \in \mathcal{Z}\right\} \text {, }
\end{aligned}
$$


for $\kappa=1$ or

$$
\begin{aligned}
& \Omega_{S+}^{2}:=\{((U, \underline{\lambda}) \in \Gamma_{1+P}: \\
&\left(\lambda_{p}[k, \ell]\right)_{p=1}^{P}\left\{\begin{array}{ll}
=-\frac{\left(\mathfrak{C}_{B_{p}}(U)[k, \ell]\right)_{p=1}^{P}}{\left\|\left(\mathfrak{C}_{B_{p}}(U)[k, \ell]\right)_{p=1}^{P}\right\|} & \text { if }\left\|\left(\mathfrak{C}_{B_{p}}(U)[k, \ell]\right)_{p=1}^{P}\right\|>0 \\
\in \mathcal{B}_{1}(0) & \text { if }\left\|\left(\mathfrak{C}_{B_{p}}(U)[k, \ell]\right)_{p=1}^{P}\right\|=0
\end{array} \text { for all } k, \ell,\right. \\
&\text { and } \left.\sum_{p=1}^{P} \overline{\widehat{\widetilde{B}}}_{p}[k, \ell] \widehat{\lambda}_{p}[k, \ell]=0, \text { for all }(k, \ell) \in \mathcal{Z}\right\},
\end{aligned}
$$

for $\kappa=2$ and

$$
\begin{aligned}
\Omega_{F+}^{G}:=\left\{(U, \underline{\lambda}) \in \Gamma_{1+P}: \widehat{U}[k, \ell]=\right. & \widehat{T}[k, \ell] \widehat{A}[k, \ell] \widehat{F}[k, \ell] \\
& \left.+\frac{1}{\beta} \widehat{T}[k, \ell] \sum_{p=1}^{P} \overline{\widehat{\widetilde{B}}}_{p}[k, \ell] \widehat{\lambda}_{p}[k, \ell], \text { for all }(k, \ell) \notin \mathcal{Z}\right\},
\end{aligned}
$$

if and only if there exists a $\underline{W}^{\dagger} \in \Gamma_{P}$ such that $\left(U^{\dagger}, \underline{W}^{\dagger}, \underline{\lambda^{\dagger}}\right)$ is a solution of Problem 3.2.9.

Proof. The first two steps are again analogous to the proof of Lemma 3.1.11. Let $\left(U_{\kappa}^{\dagger}, \lambda_{\kappa}^{\dagger}\right) \in \Gamma_{1+P}$ be in the intersection of $\Omega_{S_{+}}^{\kappa}$ and $\Omega_{F+}$, for $\kappa=1,2$ respectively. Choose $\underline{W}_{\kappa}^{\dagger}:=\underline{\mathfrak{C}}_{\underline{B}}\left(U_{\kappa}^{\dagger}\right)$, then we have by construction $\left(U_{\kappa}^{\dagger}, \underline{W}_{\kappa}^{\dagger}, \underline{\lambda}_{\kappa}^{\dagger}\right) \in \Omega_{C}$. Furthermore define

$$
\mathrm{A}_{\kappa}(\underline{W}):=|\underline{W}|_{1, \kappa}+\left\langle\underline{\lambda}_{\kappa}^{\dagger}, \underline{W}\right\rangle
$$

then we have for both choices of $\kappa \in\{1,2\}$ by the derivation of the subdifferential of $A_{\kappa}$, see [BC11] or the proof of Lemma 3.1.7 in Appendix A.2, that

$$
\underline{0} \in \partial \mathrm{A}_{\kappa}\left(\underline{\mathfrak{C}}_{\underline{B}}\left(U_{\kappa}^{\dagger}\right)\right) .
$$

Since we have by Proposition 16.34 in [BC11][p.233] that

$$
\underline{\mathfrak{C}}_{\underline{B}}\left(U_{\kappa}^{\dagger}\right)=\operatorname{prox}_{1 / \beta A_{\kappa}}\left(\underline{\mathfrak{C}}_{\underline{B}}\left(U_{\kappa}^{\dagger}\right)\right) \quad \Leftrightarrow \quad \underline{0} \in \frac{1}{\beta} \mathrm{A}_{\kappa}\left(\underline{\mathfrak{C}}_{\underline{B}}\left(U_{\kappa}^{\dagger}\right)\right),
$$

then (4.1.24) yields $\left(U_{\kappa}^{\dagger}, \underline{W}_{\kappa}^{\dagger}, \underline{\lambda}_{k}^{\dagger}\right) \in \Omega_{1}^{\kappa}$, for $\kappa=1,2$.

Now we need to show $\left(U^{\dagger}, \underline{W}^{\dagger}, \underline{\lambda}^{\dagger}\right) \in \Omega_{2}^{G}$. We will do this in the frequency domain by separately considering indices in $\mathcal{Z}$ and outside. Fix $\kappa \in\{1,2\}$, let us start with 
$(k, \ell) \notin \mathcal{Z}$. Then, for $\underline{W}_{\kappa}^{\dagger}=\left(W_{p}\right)_{p=1}^{P}$ and $\underline{\lambda}_{\kappa}^{\dagger}=\left(\lambda_{p}\right)_{p=1}^{P}$ we have

$$
\widehat{U}_{\kappa}^{\dagger}[k, \ell]=\widehat{T}[k, \ell]\left(\widehat{A}[k, \ell] \widehat{F}[k, \ell]+\frac{1}{\beta} \sum_{p=1}^{P} \widehat{\widetilde{B}}_{p}[k, \ell] \widehat{\lambda}_{p}^{\dagger}[k, \ell]\right),
$$

which is by definition of $T$ and $\mathcal{Z}$ equivalent to

$$
\left(1-\sum_{p=1}^{P} \overline{\widehat{\widetilde{B}}}_{p}[k, \ell] \widehat{B}_{p}[k, \ell]\right) \widehat{U}_{\kappa}^{\dagger}[k, \ell]=\widehat{A}[k, \ell] \widehat{F}[k, \ell]+\frac{1}{\beta} \sum_{p=1}^{P} \overline{\widehat{\widetilde{B}}_{p}[k, \ell]} \widehat{\lambda}_{p}^{\dagger}[k, \ell],
$$

yielding via $\underline{\mathfrak{C}}_{\underline{B}}\left(U_{\kappa}^{\dagger}\right)=\underline{W}_{\kappa}^{\dagger}$,

$$
\widehat{U}_{\kappa}^{\dagger}[k, \ell]=\widehat{A}[k, \ell] \widehat{F}[k, \ell]+\sum_{p=1}^{P} \overline{\widehat{\widetilde{B}}_{p}[k, \ell]}\left(\widehat{W}_{p}^{\dagger}[k, \ell]+\frac{1}{\beta} \widehat{\lambda}_{p}^{\dagger}[k, \ell]\right),
$$

for all $(k, \ell) \notin \mathcal{Z}$.

Let now $(k, \ell) \in \mathcal{Z}$. By the definition of $\Omega_{S+}^{\kappa}$ we have

$$
\sum_{p=1}^{P} \overline{\widehat{\widetilde{B}}_{p}[k, \ell]} \widehat{\lambda}_{p}^{\dagger}[k, \ell]=0
$$

yielding, by (SNEPC) and the definition of $\mathcal{Z}$ the relation

$$
\begin{aligned}
\widehat{U}_{\kappa}^{\dagger}[k, \ell] & =\sum_{p=1}^{P} \overline{\widehat{\widetilde{B}}_{p}[k, \ell]} \widehat{B}_{p}[k, \ell] \widehat{U}_{k}^{\dagger}[k, \ell]=\sum_{p=1}^{P} \overline{\widehat{\widetilde{B}}_{p}[k, \ell]} \widehat{W}_{p}^{\dagger}[k, \ell] \\
& =\underbrace{\widehat{A}[k, \ell]}_{=0 \text { by (SNEPC) }} \widehat{F}[k, \ell]+\sum_{p=1}^{P} \overline{\widehat{\widetilde{B}}_{p}[k, \ell]} \widehat{W}_{p}^{\dagger}[k, \ell]+\underbrace{\frac{1}{\beta} \sum_{p=1}^{P} \overline{\widehat{\widetilde{B}}_{p}[k, \ell]} \widehat{\lambda}_{p}^{\dagger}[k, \ell]}_{=0 \text { by construction of } \Omega_{S+}^{\kappa}} .
\end{aligned}
$$

Hence, putting both cases together, for $(k, \ell) \notin \mathcal{Z}$ see (4.1.25) and for $(k, \ell) \in \mathcal{Z}$ see (4.1.26), we have via the inverse discrete Fourier transform

$$
U_{\kappa}^{\dagger}=\mathfrak{C}_{A}(F)+\underline{\mathfrak{C}}_{\underline{\underline{B}}}^{*}\left(\underline{W}_{\kappa}^{\dagger}+\frac{1}{\beta} \underline{\lambda}_{\kappa}^{\dagger}\right)
$$

yielding $\left(U_{\kappa}^{\dagger}, \underline{W}_{\kappa}^{\dagger}, \underline{\lambda}_{\kappa}^{\dagger}\right) \in \Omega_{2}^{G}$ for $\kappa=1,2$, and thus a solution of Problem 3.2.9.

The converse is shown in a straight-forward way reversing the above calculations for a given solution $\left(U_{\kappa}^{\dagger}, \underline{W}_{\kappa}^{\dagger}, \underline{\lambda}_{\kappa}^{\dagger}\right) \in \Gamma_{1+2 P}$ of Problem 3.2.9 and $\kappa \in\{1,2\}$. 
Comparing the linear sets $\Omega_{F+}^{G}$ and $\Omega_{F}^{G}$ with one another we notice that the affine dimension increases by $|\mathcal{Z}|$ when replacing $\Omega_{F}^{G}$ by $\Omega_{F+}^{G}$. On the other hand the nonlinear set $\Omega_{S+}^{\kappa}$ loses degrees of freedom by the introduction of $|\mathcal{Z}|$ non-linear conditions as compared to $\Omega_{S}^{\kappa}$, for both $\kappa \in\{1,2\}$ respectively. The problem is now to construct $\Omega_{S+}^{0}$ in analogy to $\Omega_{S}^{0}$. How to do this, and whether a proof via the construction of Chapter 4.1.1, 4.1.2 and 4.1.3 is even possible, is open at this point. Let us give two examples of (im)possible constructions of $\Omega_{S_{+}}^{0}$ and reasons why they do not work.

- If we choose $\Omega_{S_{+}}^{0}=\Omega_{S}^{0}$ as in (4.1.10) we will have $\operatorname{dim}\left(\Omega_{S+}^{0} \cap \Omega_{F+}\right)=|\mathcal{Z}|$, hence the hypothesis of Proposition 4.1.1 is not satisfied.

- Given $\kappa=1$, if we choose

$$
\begin{aligned}
\Omega_{S+}^{0}:=\left\{(U, \underline{0}) \in \Gamma_{1+P}: \exists \xi \in \Gamma_{P}\right. \text { such that } \\
\xi_{p}[k, \ell] \begin{cases}=-1 & \text { if } \mathfrak{C}_{B_{p}}(U)[k, \ell]>0 \\
\in[-1,1] & \text { if } \mathfrak{C}_{B_{p}}(U)[k, \ell]=0, \text { for all }(k, \ell, p) \\
=1 & \text { if } \mathfrak{C}_{B_{p}}(U)[k, \ell]<0\end{cases} \\
\text { and } \left.\sum_{p=1}^{P} \overline{\widehat{\widetilde{B}}_{p}[k, \ell] \widehat{\xi}_{p}}[k, \ell]=0, \text { for all }(k, \ell) \in \mathcal{Z}\right\},
\end{aligned}
$$

then $\Omega_{S+}^{0}$ is not linear and thus does not satisfy the requirement of Proposition 4.1.1.

Note that the above described challenges also occur when trying to show the existence of a point in the intersection of the three sets $\Omega_{1}, \Omega_{2}$ and $\Omega_{C}$ of Problem 3.2.9. However, no counterexample has been found by the author leaving the existence of a fixed point under the (SNEPC) an open question.

\subsection{Convergence to a Fixed Point}

Throughout this chapter we assume that a fixed point of Algorithm 3.2.8 exists. The focus of this chapter is extending the results that have been obtained for Algorithm 3.1.5 to our more general Algorithm 3.2.8. For a short overview over the development of ADMM-algorithms see Chapter 1.4.5. In this chapter we build on proof techniques that can be found to some extend in the classical literature [Gab83, EB92, Ber82, ET99] and to more extend specifically for our discrete case in Wu and Tai [WT10], who build 
among others upon Glowinski and Le Tallec [GLT89].

Notably, for $(\underline{B}, \underline{\widetilde{B}})$ strongly admissibility and satisfying the (NEPC) we are able to construct a convex functional that is minimized by Algorithm 3.2.8, cf. Chapter 4.2.1. Hence, showing convergence can be seen as a corollary to classical results, e.g. [EB92][Theorem 8]. As a novelty, in this chapter an alternative proof will be provided, only relying on the characterization given in Problem 3.3.4. Since numerical results hint at the fact that weak admissibility is enough for convergence, the author hopes that these alternative proofs can shed some light on how to extend the results in future work to the case of weak admissibility.

To prove the convergence of Algorithm 3.1.5 in [WT10], only using the variational Problem 3.3.4, a key step is to show for a sequence of updates $\left(U^{(\tau)}, \underline{W}^{(\tau)}, \underline{\lambda}^{(\tau)}\right)_{\tau \in \mathbb{N}}$ and a fixed point $\left(U^{\dagger}, \underline{W^{\dagger}}, \underline{\lambda}^{\dagger}\right) \in \Gamma_{1+2 P}$ that the sequences

$$
\left(\left\|\underline{\lambda}^{(\tau)}-\underline{\lambda}^{\dagger}\right\|^{2}\right)_{\tau \in \mathbb{N}} \text { and }\left(\left\|\underline{\mathfrak{C}}_{\underline{B}}\left(U^{(\tau)}-U^{\dagger}\right)\right\|^{2}\right)_{\tau \in \mathbb{N}},
$$

are bounded. This is done using variational inequalities given by the updating steps of $U^{(\tau)}$ and $\underline{W}^{(\tau)}$, which are minimizations in themselves, cf. Problem 3.3.4. In order for these inequalities to hold in the more general setting we will need that the input filters $(\underline{B}, \underline{\widetilde{B}}) \in \Gamma_{2 P}$ are weakly admissible and that

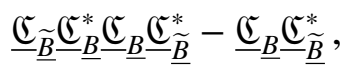

is a negative semi-definite operator. Let us prove that this is exactly the case if $(\underline{B}, \underline{\widetilde{B}})$ are strongly admissible and satisfy the (NEPC).

Lemma 4.2.1. Let $(\underline{B}, \underline{\widetilde{B}}) \in \Gamma_{2 P}$ be weakly admissible. Then $(\underline{B}, \underline{\widetilde{B}})$ are strongly admissible and satisfy the $(N E P C)$ if and only if $\underline{\mathfrak{C}}_{\widetilde{\underline{B}}} \underline{\mathfrak{C}_{\underline{B}}^{*}} \underline{\mathfrak{C}}_{\underline{B}} \underline{\mathfrak{C}_{\underline{B}}^{*}}-\underline{\mathfrak{C}}_{\underline{B}} \underline{\mathfrak{C}}_{\underline{\underline{B}}}^{*}$ is negative semi-definite.

Proof. Assume $(\underline{B}, \underline{\widetilde{B}})$ are strongly admissible and satisfy the (NEPC). Let $\underline{W} \in \Gamma_{P}$, then

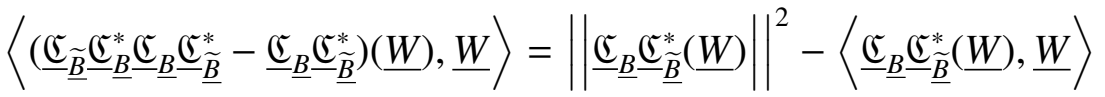

$$
\begin{aligned}
& =\left\|\underline{\mathfrak{C}}_{\underline{B}} \underline{\mathfrak{C}}_{\underline{\underline{B}}}^{*}(\underline{W})\right\|^{2}-\left\|\left(\underline{\mathfrak{C}}_{\underline{B}} \underline{\mathfrak{C}}_{\underline{\widetilde{B}}}^{*}\right)^{1 / 2}(\underline{W})\right\|^{2} \\
& \leq\left(\rho\left(\left(\underline{\mathfrak{C}}_{\underline{B}} \underline{\mathfrak{C}}_{\underline{\underline{B}}}^{*}\right)^{1 / 2}\right)^{2}-1\right)\left\|\left(\underline{\mathfrak{C}_{\underline{B}}} \underline{\widetilde{C}}_{\underline{\underline{\underline{C}}}}^{*}\right)^{1 / 2}(\underline{W})\right\|^{2} \\
& \leq 0 \text {, }
\end{aligned}
$$


where the second equality holds since by Corollary 3.2.6 the principal square-root $\left(\underline{\mathfrak{C}}_{\underline{B}} \underline{\mathfrak{C}}_{\underline{\underline{B}}}^{*}\right)^{1 / 2}$ exists and is by Lemma 3.2.5 self-adjoint. Moreover, by Lemma 3.2.5 and Corrollary 3.2.6 and the (NEPC) the maximal eigenvalue of $\left(\underline{\mathfrak{C}}_{\underline{B}} \underline{\mathfrak{C}}_{\underline{\underline{B}}}^{*}\right)^{1 / 2}$ is smaller or equal than 1 , yielding the last inequality.

For the converse direction consider two cases.

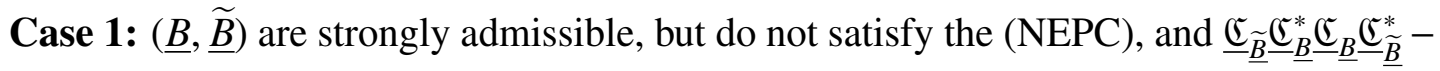
$\underline{\mathfrak{C}}_{\underline{B}} \underline{\mathfrak{C}}_{\widetilde{B}}^{*}$ is negative semi-definite.

By strong admissibility we have that all eigenvalues of $\underline{\mathfrak{C}}_{\underline{B}} \underline{\mathfrak{C}}_{\widetilde{B}}^{*}$ lie in $\mathbb{R}_{+}$. Hence, by not satisfying the (NEPC), there exists an eigenmatrix $\underline{W} \in \Gamma_{P}$ of $\underline{\mathfrak{C}}_{\underline{B}} \underline{\mathfrak{C}}_{\widetilde{B}}^{*}$ to an eigenvalue greater than 1. Plugging this $\underline{W}$ into the calculation 4.2.1 yields a contradiction to the

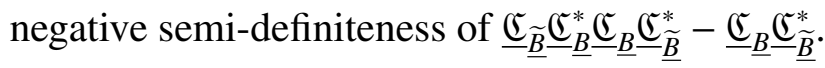

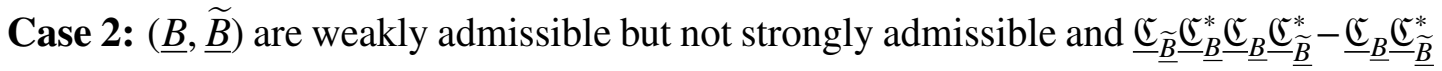
is negative semi-definite.

By weak admissibility there exists $\underline{Y} \in \Gamma_{P}$ such that

$$
\widehat{\widetilde{B}}_{p}[k, \ell]=\widehat{Y}_{p}[k, \ell] \widehat{B}_{p}[k, \ell]
$$

for all $0 \leq k \leq n-1$ and $0 \leq \ell \leq m-1$ and $1 \leq p \leq P$. Moreover, since $(\underline{B}, \underline{\widetilde{B}})$ are not strongly admissible there exists a $k_{0} \in\{0,1, \ldots, n-1\}$ and $\ell_{0} \in\{0,1, \ldots, m-1\}$ and $p_{0} \neq p_{1} \in\{1,2, \ldots, P\}$ such that

$$
\widehat{Y}_{p_{0}}\left[k_{0}, \ell_{0}\right] \neq \widehat{Y}_{p_{1}}\left[k_{0}, \ell_{0}\right] \quad \text { and } \quad \widehat{B}_{p_{0}}\left[k_{0}, \ell_{0}\right], \widehat{B}_{p_{1}}\left[k_{0}, \ell_{0}\right] \neq 0
$$

otherwise there would exist an admissibility matrix $Y \in \mathbb{R}^{n \times m}$, cf. Definition 3.2.1.

Claim A: $\operatorname{ker}\left(\underline{\mathfrak{C}}_{\widetilde{B}}^{*}\right)$ and $\operatorname{Im}\left(\underline{\mathfrak{C}}_{\underline{B}}\right)$ are not orthogonal subspaces of $\Gamma_{P}$.

First, define $\underline{W} \in \Gamma_{P}$ by

$$
\widehat{W}_{p}[k, \ell]:= \begin{cases}\widehat{\widetilde{B}}_{p_{1}}\left[k_{0}, \ell_{0}\right] & \text { if }(k, \ell, p)=\left(k_{0}, \ell_{0}, p_{0}\right) \\ \widehat{\widetilde{B}}_{p_{0}}\left[k_{0}, \ell_{0}\right] & \text { if }(k, \ell, p)=\left(k_{0}, \ell_{0}, p_{1}\right) . \\ 0 & \text { else }\end{cases}
$$

Then, 


$$
\begin{aligned}
\underline{\underline{\mathbb{C}}_{\widetilde{\widetilde{B}}}^{*}(\underline{W})[k, \ell]} & =\sum_{p=1}^{P} \widehat{\widetilde{B}}_{p}[k, \ell] \widehat{W}_{p}[k, \ell] \\
& = \begin{cases}\widehat{\widetilde{B}}_{p_{0}}\left[k_{0}, \ell_{0}\right] \widehat{\widetilde{B}}_{p_{1}}\left[k_{0}, \ell_{0}\right]-\widehat{\widetilde{B}}_{p_{1}}\left[k_{0}, \ell_{0}\right] \widehat{\widetilde{B}}_{p_{0}}\left[k_{0}, \ell_{0}\right] & \text { if }(k, \ell)=\left(k_{0}, \ell_{0}\right) \\
0 & \text { else }\end{cases} \\
& =0,
\end{aligned}
$$

yielding $\underline{W} \in \operatorname{ker}\left(\underline{\mathfrak{C}}_{\underline{\underline{B}}}^{*}\right)$, in particular $\underline{W} \in \operatorname{ker}\left(\underline{\mathfrak{C}}_{\underline{B}} \underline{\mathfrak{C}}_{\underline{\widetilde{B}}}^{*}\right)$. Secondly, given

$$
\widehat{U}^{\prime}[k, \ell]:=\left\{\begin{array}{ll}
1 & \text { if }(k, \ell)=\left(k_{0}, \ell_{0}\right) \\
0 & \text { else }
\end{array},\right.
$$

define $\underline{W}^{\prime} \in \Gamma_{P}$ as $\underline{W}^{\prime}=\underline{\mathfrak{C}}_{\underline{B}}\left(U^{\prime}\right) \in \operatorname{Im}\left(\underline{\mathfrak{C}}_{\underline{B}}\right)$. Via this choice we have

$$
\widehat{W}_{p}^{\prime}[k, \ell]=\left\{\begin{array}{ll}
\widehat{B}_{p}\left[k_{0}, \ell_{0}\right] & \text { if }(k, \ell)=\left(k_{0}, \ell_{0}\right) \\
0 & \text { else }
\end{array} .\right.
$$

We obtain,

$$
\begin{aligned}
\frac{1}{m n}\left\langle\underline{W}, \underline{W^{\prime}}\right\rangle & =\frac{1}{m n} \sum_{p=1}^{P}\left\langle W_{p}, W_{p}^{\prime}\right\rangle=\sum_{p=1}^{P}\left\langle\widehat{W}_{p}, \widehat{W}_{p}^{\prime}\right\rangle \\
& = \begin{cases}\widehat{W}_{p_{0}}\left[k_{0}, \ell_{0}\right] \overline{\widehat{W}_{p_{0}}^{\prime}\left[k_{0}, \ell_{0}\right]}+\widehat{W}_{p_{1}}\left[k_{0}, \ell_{0}\right] \overline{\widehat{W}_{p_{1}}^{\prime}\left[k_{0}, \ell_{0}\right]} & \text { if }(k, \ell)=\left(k_{0}, \ell_{0}\right) \\
0 & \text { else }\end{cases} \\
& = \begin{cases}\widehat{\widetilde{B}}_{p_{1}}\left[k_{0}, \ell_{0}\right] \overline{\widehat{B}_{p_{0}}\left[k_{0}, \ell_{0}\right]}-\widehat{\widetilde{B}}_{p_{0}}\left[k_{0}, \ell_{0}\right] \overline{\widehat{B}_{p_{1}}\left[k_{0}, \ell_{0}\right]} & \text { if }(k, \ell)=\left(k_{0}, \ell_{0}\right) \\
0 & \text { else }\end{cases}
\end{aligned}
$$

where the second equality is due to the Lemma of Plancherel, cf. Lemma 2.1.9. Assume that $\underline{W}$ and $\underline{W^{\prime}}$ were orthogonal, than the above calculation yields

$$
\widehat{\widetilde{B}}_{p_{1}}\left[k_{0}, \ell_{0}\right] \overline{\widehat{B}_{p_{0}}\left[k_{0}, \ell_{0}\right]}-\widehat{\widetilde{B}}_{p_{0}}\left[k_{0}, \ell_{0}\right] \overline{\widehat{B}_{p_{1}}\left[k_{0}, \ell_{0}\right]}=0 .
$$

Hence,

$$
\begin{aligned}
& 0=\operatorname{Re}\left(\widehat{\widetilde{B}}_{p_{1}}\left[k_{0}, \ell_{0}\right] \overline{\widehat{B}_{p_{0}}\left[k_{0}, \ell_{0}\right]}-\widehat{\widetilde{B}}_{p_{0}}\left[k_{0}, \ell_{0}\right] \overline{\widehat{B}_{p_{1}}\left[k_{0}, \ell_{0}\right]}\right) \\
& =\operatorname{Re}\left(\widehat{Y}_{p_{1}}\left[k_{0}, \ell_{0}\right]^{-1} \widehat{B}_{p_{1}}\left[k_{0}, \ell_{0}\right] \overline{\widehat{B}_{p_{0}}\left[k_{0}, \ell_{0}\right]}-\widehat{Y}_{p_{0}}\left[k_{0}, \ell_{0}\right]^{-1} \widehat{B}_{p_{0}}\left[k_{0}, \ell_{0}\right] \overline{\widehat{B}_{p_{1}}\left[k_{0}, \ell_{0}\right]}\right) \\
& =\left(\widehat{Y}_{p_{0}}\left[k_{0}, \ell_{0}\right]^{-1}-\widehat{Y}_{p_{1}}\left[k_{0}, \ell_{0}\right]^{-1}\right) \operatorname{Re}\left(\widehat{B}_{p_{1}}\left[k_{0}, \ell_{0}\right] \overline{\widehat{B}_{p_{0}}\left[k_{0}, \ell_{0}\right]}\right),
\end{aligned}
$$


yielding by $\widehat{Y}_{p_{0}}\left[k_{0}, \ell_{0}\right] \neq \widehat{Y}_{p_{1}}\left[k_{0}, \ell_{0}\right]$ that $\operatorname{Re}\left(\widehat{B}_{p_{1}}\left[k_{0}, \ell_{0}\right] \overline{\widehat{B}_{p_{0}}\left[k_{0}, \ell_{0}\right]}\right)=0$.

On the other hand,

$$
\begin{aligned}
0 & =\operatorname{Im}\left(\widehat{\widetilde{B}}_{p_{1}}\left[k_{0}, \ell_{0}\right] \overline{\widehat{B}_{p_{0}}\left[k_{0}, \ell_{0}\right]}-\widehat{\widetilde{B}}_{p_{0}}\left[k_{0}, \ell_{0}\right] \overline{\widehat{B}_{p_{1}}\left[k_{0}, \ell_{0}\right]}\right) \\
& =\operatorname{Im}\left(\widehat{Y}_{p_{1}}\left[k_{0}, \ell_{0}\right]^{-1} \widehat{B}_{p_{1}}\left[k_{0}, \ell_{0}\right] \overline{\widehat{B}_{p_{0}}\left[k_{0}, \ell_{0}\right]}-\widehat{Y}_{p_{0}}\left[k_{0}, \ell_{0}\right]^{-1} \widehat{B}_{p_{0}}\left[k_{0}, \ell_{0}\right] \overline{\widehat{B}_{p_{1}}\left[k_{0}, \ell_{0}\right]}\right) \\
& =\left(\widehat{Y}_{p_{0}}\left[k_{0}, \ell_{0}\right]^{-1}+\widehat{Y}_{p_{1}}\left[k_{0}, \ell_{0}\right]^{-1}\right) \operatorname{Im}\left(\widehat{B}_{p_{1}}\left[k_{0}, \ell_{0}\right] \overline{\widehat{B}_{p_{0}}\left[k_{0}, \ell_{0}\right]}\right),
\end{aligned}
$$

which yields by $\widehat{Y}_{p_{0}}\left[k_{0}, \ell_{0}\right], \widehat{Y}_{p_{1}}\left[k_{0}, \ell_{0}\right] \in \mathbb{R}_{+}$that $\operatorname{Im}\left(\widehat{B}_{p_{1}}\left[k_{0}, \ell_{0}\right] \overline{\widehat{B}_{p_{0}}\left[k_{0}, \ell_{0}\right]}\right)=0$. We obtain at once,

$$
\widehat{B}_{p_{1}}\left[k_{0}, \ell_{0}\right] \widehat{\widehat{B}_{p_{0}}\left[k_{0}, \ell_{0}\right]}=0,
$$

giving a contradiction to the second part of (4.2.2). We have thus shown that $\operatorname{ker}\left(\underline{\mathbb{C}}_{\underline{\underline{B}}}^{*}\right)$ and $\operatorname{Im}\left(\underline{\mathfrak{C}}_{\underline{B}}\right)$ are not orthogonal to each other.

Claim B: $\underline{W}^{\prime}=\underline{\mathfrak{C}}_{\underline{B}}\left(U^{\prime}\right)$, given via (4.2.3), is an eigen-family of matrices with respect to $\underline{\mathfrak{C}}_{\underline{B}} \underline{\mathfrak{C}_{\underline{B}}^{*}}$ to an eigenvalue $\rho>0$.

Compute,

$$
\begin{aligned}
\mathfrak{F}\left(\underline{\mathfrak{E}}_{\underline{\widetilde{B}}}^{*} \underline{\mathfrak{C}}_{\underline{B}}\left(U^{\prime}\right)\right)[k, \ell] & = \begin{cases}\sum_{p=1}^{P} \widehat{Y}_{p}[k, \ell]\left|\widehat{B}_{p}[k, \ell]\right|^{2} & \text { if }(k, \ell)=\left(k_{0}, \ell_{0}\right) \\
0 & \text { else }\end{cases} \\
& =\underbrace{\left(\sum_{p=1}^{P} \widehat{Y}_{p}[k, \ell]\left|\widehat{B}_{p}[k, \ell]\right|^{2}\right)}_{=: \rho} \widehat{U}^{\prime},
\end{aligned}
$$

giving that $U^{\dagger}$ is an eigenmatrix with respect to $\underline{\mathfrak{C}}_{\underline{\underline{B}}}^{*} \underline{\underline{C}} \underline{\underline{B}}$ to an eigenvalue $\rho>0$. Via,

$$
\underline{\mathfrak{C}}_{\underline{B}} \underline{\mathfrak{C}}_{\underline{\underline{S}}}^{*}\left(\underline{W}^{\prime}\right)=\underline{\mathfrak{C}}_{\underline{B}} \underline{\mathfrak{C}}_{\underline{\underline{C}}}^{*} \underline{\mathfrak{C}}_{\underline{B}}\left(U^{\prime}\right)=\underline{\mathfrak{C}}_{\underline{B}}\left(\rho U^{\prime}\right)=\rho \underline{W}^{\prime},
$$

we have that the same holds true for $\underline{W}^{\prime}$ with respect to $\underline{\mathfrak{C}}_{\underline{B}} \underline{\mathfrak{C}}_{\underline{\underline{B}}}^{*}$. In particular $\underline{W}^{\prime} \notin$ $\operatorname{ker}\left(\underline{\mathfrak{C}}_{\underline{B}} \underline{\mathfrak{C}_{\underline{B}}^{*}}\right)$

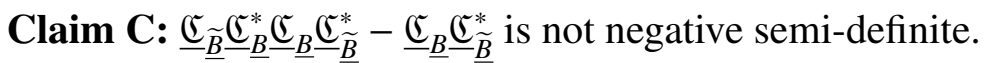

Via rescaling define

$$
\underline{V}:=\frac{\underline{W}}{\|\underline{W}\|} \quad \text { and } \quad \underline{V}^{\prime}:=\frac{\underline{W}^{\prime}}{\left\|\underline{W^{\prime}}\right\|} \text {. }
$$

Then, $\underline{V}^{\prime}$ is an eigen-family of matrices with respect to $\underline{\mathbb{C}}_{\underline{B}} \underline{\mathfrak{C}}_{\vec{B}}^{*}$ to an eigenvalue $\rho>0$ as 
well as

$$
\|\underline{V}\|=1=\left\|\underline{V^{\prime}}\right\| \quad \text { and } \quad\left\langle\underline{V^{\prime}}, \underline{V}\right\rangle=\gamma \in(0,1) .
$$

Choose $R<(\rho-1) / \gamma$, then

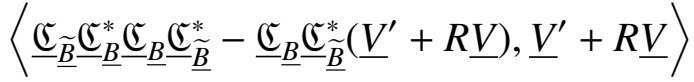

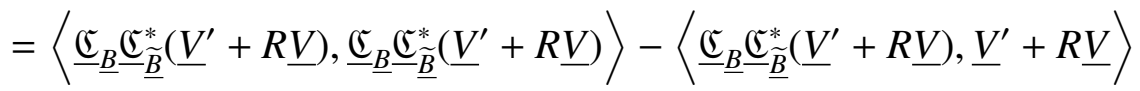

$$
\begin{aligned}
& =\left\|\underline{\underline{\underline{C}}} \underline{\underline{\underline{E}}} \underline{\underline{\widetilde{B}}} *\left(\underline{V}^{\prime}\right)\right\|^{2}-\rho\left\|\underline{V^{\prime}}\right\|^{2}-\rho R\left\langle\underline{V^{\prime}}, \underline{V}\right\rangle \\
& =\rho^{2}\left\|\underline{V}^{\prime}\right\|^{2}-\rho-\rho \gamma R=\rho(\rho-1-\gamma R) \\
& >\rho\left(\rho-1-\gamma \frac{\rho-1}{\gamma}\right)=0,
\end{aligned}
$$

where the second equality holds true by $\underline{V} \in \operatorname{ker}\left(\underline{\mathfrak{C}}_{\underline{B}} \underline{\mathfrak{C}}_{\widetilde{B}}^{*}\right)$. This yields a contradiction to

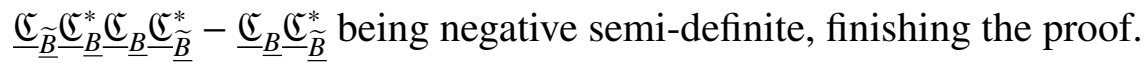

First, in Chapter 4.2.1 the reduction of Problem 3.2.9 in the case of strong admissibility and the (NEPC) to a minimization problem is discussed. Second, we generalize the proof of [WT10] to obtain an alternative proof of convergence using only Problem 3.3.4. To this end, the following roadmap is used.

(i) In Chapter 4.2.2 we prove necessary, technical inequalities.

(ii) In Chapter 4.2.3 we deduce the convergence of Algorithm 3.2.8 given that there exists a fixed point and $(\underline{B}, \underline{\widetilde{B}})$ are strongly admissible and satisfy the (NEPC).

\subsubsection{A Remark on Admissibility}

Let us consider the case that $(\underline{B}, \underline{\widetilde{B}})$ are strongly admissible and satisfy the (NEPC). We detail below how to obtain a functional whose minimizers are given by the solutions of Problem 3.2.9. Note, that generalizing to strong admissibility is equivalent to generalizing the balancing parameter $\mu \in \mathbb{R}_{+}$in (1.3.2) to a matrix convolution, with convolution matrix $M \in \mathbb{R}^{n \times m}$. The latter generalization has been proposed by Aujol and Gilboa in [AG06]. Independently, various specific $M \in \mathbb{R}^{n \times m}$ were employed, for example by Osher et al. [OSV03], Garnett et al. [GLMV07] and Buades et al. [BLMV10].

Let $F \in \mathbb{R}^{n \times m}, \beta \in \mathbb{R}_{+}, \kappa \in\{1,2\}$ and let $(A, \underline{B}, \underline{\widetilde{B}}) \in \Gamma_{1+2 P}$ be input filters, such that $(\underline{B}, \underline{\widetilde{B}})$ are strongly admissible with strong admissibly matrix $Y \in \mathbb{R}^{n \times m}$ and satisfy the (NEPC), cf. Defintion 3.2.1. Furthermore, assume we have

$$
\mathfrak{C}_{A}=\mathfrak{C}-\underline{\mathfrak{C}}_{\underline{\underline{B}}}^{*} \underline{\mathfrak{C}}_{\underline{B}} .
$$


Then define $M \in \mathbb{R}^{n \times m}$ such that it gives rise to the convolution operator

$$
\mathfrak{C}_{M}=\beta \mathfrak{C}_{Y}^{-1}\left(\mathfrak{\mathbb { E }}-\underline{\mathfrak{C}}_{\underline{\underline{B}}}^{*} \underline{\mathfrak{C}}_{\underline{B}}\right)=\beta \mathfrak{C}_{Y}^{-1}-\beta \underline{\mathfrak{C}}_{\underline{B}}^{*} \underline{\mathfrak{C}}_{\underline{B}} .
$$

Note that $\mathfrak{C}_{M}$ is well defined and a self-adjoint operator by definition of $Y \in \mathbb{R}^{n \times m}$. Thus, $\mathfrak{C}_{M}$ has only real eigenvalues that are greater or equal to 0 , making it positive semidefinite, yielding the existence of a principal square root $\left(\mathfrak{C}_{M}\right)^{1 / 2}$, which is again a convolution operator. Let its convolution matrix be denoted by $\widetilde{M} \in \mathbb{R}^{n \times m}$, then $\mathfrak{C}_{\widetilde{M}}$ is again self-adjoint. Define the convex functional

$$
\mathcal{J}_{\widetilde{M}}(U):=\left|\underline{\mathfrak{C}}_{\underline{B}}(U)\right|_{1, \kappa}+\frac{1}{2}\left\|\mathfrak{C}_{\widetilde{M}}(F-U)\right\|^{2}
$$

and its augmented Lagrangian,

$\mathcal{J}_{\mathrm{AL}, \widetilde{M}}(U, \underline{W}, \underline{\lambda}):=|\underline{W}|_{1, \kappa}+\left.\frac{1}{2}|| \mathfrak{C}_{\widetilde{M}}(F-U)\right|^{2}+\frac{\beta}{2}|| \underline{W}-\left.\underline{\mathfrak{C}}_{\underline{B}}(U)\right|^{2}+\left\langle\underline{\lambda}, \underline{W}-\underline{\mathfrak{C}}_{\underline{B}}(U)\right\rangle$.

Lemma 4.2.2. Let $F \in \mathbb{R}^{n \times m}, \beta \in \mathbb{R}_{+}, \kappa \in\{1,2\}$ and let $(A, \underline{B}, \underline{\widetilde{B}})$ be input filters such that $\mathfrak{C}_{A}=\mathbb{\mathfrak { E }}-\underline{\mathfrak{C}}_{\widetilde{\underline{B}}}^{*} \underline{\mathfrak{C}}_{\underline{B}}$ holds and $(\underline{B}, \underline{\widetilde{B}})$ are strongly admissible and satisfy the (NEPC). Let furthermore, $\mathfrak{C}_{M}$ and $\mathcal{J}_{A L, \widetilde{M}}$ be defined as in (4.2.4) and (4.2.6), respectively. Then $\left(U^{\dagger}, \underline{W}^{\dagger}, \underline{\lambda}^{\dagger}\right) \in \Gamma_{1+2 P}$ is a solution of Problem 3.2.9 if and only if it is a saddle point of $\mathcal{J}_{A L, \widetilde{M}}$.

Proof. Let $\left(U^{\dagger}, \underline{W}^{\dagger}, \underline{\lambda}^{\dagger}\right)$ be a solution of Problem 3.2.9.

First, by $\left(U^{\dagger}, \underline{W}^{\dagger}, \underline{\lambda}^{\dagger}\right) \in \Omega_{C}$ we have that $\underline{\lambda} \mapsto\left\langle\underline{\lambda}, \underline{W}^{\dagger}-\underline{\mathfrak{C}}_{B}\left(U^{\dagger}\right)\right\rangle$ is constant and thus $\underline{\lambda}^{\dagger} \operatorname{maximizes}(4.2 .6)$.

Secondly, by (i) of Lemma 3.1.7, $\underline{W} \mapsto \mathcal{J}_{\mathrm{AL}, \widetilde{M}}\left(U^{\dagger}, \underline{W}, \underline{\lambda^{\dagger}}\right)$ is minimal for $\underline{W}=\underline{W^{\dagger}}$.

Last, since $U \mapsto \mathcal{J}_{\mathrm{AL}, \widetilde{M}}\left(U, \underline{W}^{\dagger}, \underline{\lambda}^{\dagger}\right)$ is a differentiable, convex functional a necessary and sufficient condition on a minimizer $U^{\dagger}$ is given by $\frac{\partial}{\partial U} \mathcal{J}_{\mathrm{AL}, \widetilde{M}}\left(U^{\dagger}, \underline{W^{\dagger}}, \underline{\lambda}^{\dagger}\right)=0$ (see for example Boyd and Vandenberghe [BV04][p.136ff.]). Computing the derivative with respect to $U$ and setting it zero we obtain

$$
\begin{aligned}
& 0=\mathfrak{C}_{\widetilde{M}}^{*} \mathfrak{C}_{\widetilde{M}}(U)-\mathfrak{C}_{\widetilde{M}}^{*} \mathfrak{C}_{\widetilde{M}}(F)+\beta \underline{\mathfrak{C}}_{\underline{B}}^{*} \underline{\mathfrak{C}}_{\underline{B}}(U)-\beta \underline{\mathfrak{C}}_{\underline{B}}^{*}\left(\underline{W}^{\dagger}\right)-\underline{\mathfrak{C}}_{\underline{B}}^{*}\left(\underline{\lambda}^{\dagger}\right) \\
& \Leftrightarrow \quad\left(\mathfrak{C}_{M}+\beta \underline{\mathfrak{C}}_{\underline{B}}^{*} \underline{\mathfrak{C}_{B}}\right)(U)=\mathfrak{C}_{M}(F)+\beta \underline{\mathfrak{C}}_{\underline{B}}^{*}\left(\underline{W}^{\dagger}+1 / \beta \underline{\lambda^{\dagger}}\right)
\end{aligned}
$$

We have,

$$
\mathfrak{C}_{M}+\beta \underline{\mathfrak{C}}_{\underline{B}}^{*} \underline{\mathfrak{C}}_{\underline{B}}=\beta \mathfrak{C}_{Y}^{-1},
$$

hence,

$$
U=\frac{1}{\beta} \mathfrak{C}_{Y} \mathfrak{C}_{M}(F)+\underline{\mathfrak{C}}_{\underline{\underline{\widetilde{B}}}}^{*}\left(\underline{W}^{\dagger}+1 / \beta \underline{\lambda^{\dagger}}\right),
$$


with $\frac{1}{\beta} \mathfrak{C}_{Y} \mathfrak{C}_{M}=\beta / \beta\left(\mathfrak{E}-\underline{\mathfrak{C}}_{\widetilde{B}}^{*} \underline{\mathfrak{C}}_{\underline{B}}\right)=\mathfrak{C}_{A}$ we obtain by $\left(U^{\dagger}, \underline{W}^{\dagger}, \underline{\lambda}^{\dagger}\right) \in \Omega_{2}^{G}$ that $U^{\dagger}$ minimizes $U \mapsto \mathcal{J}_{\mathrm{AL}, \widetilde{M}}\left(U, \underline{W}^{\dagger}, \underline{\lambda}^{\dagger}\right)$. Hence, $\left(U^{\dagger}, \underline{W}^{\dagger}, \underline{\lambda}^{\dagger}\right)$ is a saddle point of $\mathcal{J}_{\mathrm{AL}, \widetilde{M}}$.

Vice versa let $\left(U^{\dagger}, \underline{W}^{\dagger}, \underline{\lambda}^{\dagger}\right)$ be a saddle point of (4.2.6), since $\mathcal{J}_{\mathrm{AL}, \widetilde{M}}$ is linear in $\underline{\lambda}$ and maximized by $\underline{\lambda}^{\dagger}$ for fixed $U^{\dagger}, \underline{W}^{\dagger}$, the linear term vanishes. Hence, $\left(U^{\dagger}, \underline{W}^{\dagger}, \underline{\lambda}^{\dagger}\right) \in$ $\Omega_{C}$. By (i) of Lemma 3.1.7 and $\underline{W}^{\dagger}$ minimizes $\underline{W} \mapsto \mathcal{J}_{\mathrm{AL}, \widetilde{M}}\left(U^{\dagger}, \underline{W}, \underline{\lambda}^{\dagger}\right)$ we have $\left(U^{\dagger}, \underline{W}^{\dagger}, \underline{\lambda}^{\dagger}\right) \in \Omega_{1}^{\kappa}$. Last, by computing the derivative done above and $U^{\dagger}$ minimizing $U \mapsto \mathcal{J}_{\mathrm{AL}, \widetilde{M}}\left(U, \underline{W}^{\dagger}, \underline{\lambda}^{\dagger}\right)$ we have $\left(U^{\dagger}, \underline{W}^{\dagger}, \underline{\lambda}^{\dagger}\right) \in \Omega_{2}^{G}$. Thus, $\left(U^{\dagger}, \underline{W^{\dagger}}, \underline{\lambda}^{\dagger}\right) \in \Omega_{1}^{\kappa} \cap \Omega_{2}^{G} \cap$ $\Omega_{C}$.

We obtain the following Remarks.

Remark 4.2.3. (i) A saddle-point of (4.2.6) can be shown to be a minimizer of (4.2.5), in analogy to Lemma 3.1.4. Moreover, $\mathcal{J}_{\widetilde{M}}$ of (4.2.5) is a convex function, and strictly convex if and only if $\operatorname{ker}\left(\mathfrak{C}_{\widetilde{M}}\right)=\{0\}$. Since $\mathfrak{C}_{M}$, and hence $\mathfrak{C}_{\widetilde{M}}$, has fullrank if and only if the $(C P C)$ is satisfied for $(\underline{B}, \underline{\widetilde{B}})$ - by definition of the strong admissibility matrix $Y$-, enforcing the (CPC) yields the existence of a unique minimizer.

(ii) Let $(\underline{B}, \underline{\widetilde{B}})$ be strongly admissible and satisfy the (NEPC). Then, Algorithm 3.2.8 is of ADMM structure with respect to computing a saddlepoint of the augmented Lagrangian functional $\mathcal{J}_{A L, \widetilde{M}}$. Hence, its convergence analysis might be derived more straight forward from classical results such as the ones in Eckstein and Bertsekas [EB92][Theorem 8].

\subsubsection{Preliminary Lemmas}

In order to later prove convergence of Algorithm 3.2.8, we first need to prove some technical inequalities, concerning among others the newly introduced $\underline{\underline{c}}_{\underline{C}}$ of (3.3.3). For given input filters $(A, \underline{B}, \underline{\widetilde{B}}) \in \Gamma_{1+2 P}$, let us reiterate here the construction of $\underline{C}$. We first constructed in (3.3.2) the matrix-family $\underline{G}=\left(G_{p}\right)_{p=1}^{P}$ in the frequency domain by

$$
\widehat{G}_{p}[k, \ell]:= \begin{cases}\left(\overline{\widehat{B}_{p}[k, \ell]}\right)^{-1} \overline{\widehat{\widetilde{B}}_{p}[k, \ell]} & \text { if } \overline{\widehat{B}_{p}[k, \ell]} \neq 0 \\ 1 & \text { else }\end{cases}
$$

for $0 \leq k \leq n-1$ and $0 \leq \ell \leq m-1$. Thus an entry-wise matrix-family convolution is defined by $\underline{\underline{\mathfrak{C}}}_{\underline{G}}=\Gamma_{P} \rightarrow \Gamma_{P}$. Given $\underline{\underline{\mathfrak{C}}}_{\underline{G}}$, we defined $\underline{C} \in \Gamma_{P}$, as the matrix-family, giving 
rise to the entry-wise matrix-family convolution $\underline{\underline{\mathfrak{C}}}_{\underline{C}}=\left(\mathfrak{C}_{C_{p}}\right)_{p=1}^{P}: \Gamma_{P} \rightarrow \Gamma_{P}$ given by

$$
\mathfrak{C}_{C_{p}}:=\left(\mathfrak{E}+\underline{\mathfrak{C}}_{\underline{B}}^{*} \underline{\mathfrak{C}_{B}}\right) \mathfrak{C}_{G_{p}} \quad \text { for } 1 \leq p \leq P .
$$

Now we are ready to present the auxiliary Lemma, needed later.

Lemma 4.2.4. Let $(\underline{B}, \underline{\widetilde{B}}) \in \Gamma_{2 P}$ be strongly admissible and satisfy the (NEPC), and let $\underline{G}$ and $\underline{C}$ be defined as in (3.3.2) and (3.3.3), respectively. Let $V \in \mathbb{R}^{n \times m}$ and $\underline{H} \in \Gamma_{P}$ be such that

$$
\underline{\mathfrak{C}}_{\underline{\widetilde{B}}}^{*}(\underline{H})=V .
$$

Then,

$$
\left\langle\left(\underline{\underline{\mathfrak{C}}}_{\underline{C}}-\underline{\underline{\mathfrak{E}}}\right)(\underline{H}), \underline{\underline{C}}_{\underline{B}}(V)\right\rangle-\|V\|^{2} \leq 0,
$$

where $\underline{\underline{\mathfrak{E}}}$ is the identity on $\Gamma_{P}$.

Proof. Define the matrix

$$
Q[k, \ell]:=\sum_{p=1}^{P}\left\|\widehat{B}_{p}[k, \ell]\right\|^{2},
$$

for $0 \leq k \leq n-1$ and $0 \leq \ell \leq m-1$. Then $Q$ gives rise to $\underline{Q}=(\underbrace{Q, \ldots, Q}_{P \text {-times }})$ and subsequently to the entry-wise matrix-convolution

$$
\underline{\underline{\mathfrak{C}}}_{\underline{Q}}: \Gamma_{P} \rightarrow \Gamma_{P} ; \quad\left(W_{p}\right)_{p=1}^{P} \mapsto\left(\underline{\mathfrak{C}}_{\underline{B}}^{*} \underline{\mathfrak{C}}_{\underline{B}}\left(W_{p}\right)\right)_{p=1}^{P} .
$$

Consider,

$$
\begin{aligned}
& \left\langle\left(\underline{\underline{\mathfrak{C}}}_{\underline{C}}-\underline{\underline{\mathfrak{E}}}\right)(\underline{H}), \underline{\mathfrak{C}}_{\underline{B}}(V)\right\rangle-\|V\|^{2}=\underbrace{\left\langle\underline{\underline{\mathfrak{C}}}_{\underline{G}}(\underline{H}), \underline{\mathfrak{C}}_{\underline{B}}(V)\right\rangle}_{\mathrm{I}}+\underbrace{\left\langle\underline{\underline{\mathfrak{C}}}_{\underline{Q}} \underline{\underline{\mathfrak{C}}}_{\underline{G}}(\underline{H}), \underline{\mathfrak{C}}_{\underline{B}}(V)\right\rangle} \\
& -\underbrace{\left\langle\underline{H}, \underline{\mathfrak{C}}_{\underline{B}}(V)\right\rangle}_{\mathrm{III}}-\underbrace{\|V\|^{2}}_{\mathrm{IV}},
\end{aligned}
$$

derived from the definition of $\underline{\underline{\underline{C}}}_{\underline{C}}$ in (3.3.3).

Starting with term I of (4.2.10) we obtain, using the definition of $\underline{\underline{C}}_{\underline{G}}$, Remark 3.3.1 and Equation (4.2.8), that

$$
\left\langle\underline{\underline{\mathfrak{C}}}_{\underline{G}}(\underline{H}), \underline{\mathfrak{C}}_{\underline{B}}(V)\right\rangle=\left\langle\underline{\mathfrak{C}}_{\underline{B}}^{*} \underline{\mathfrak{C}}_{\underline{G}}(\underline{H}), V\right\rangle=\left\langle\underline{\mathfrak{C}}_{\underline{\underline{\widetilde{C}}}}^{*}(\underline{H}), V\right\rangle=\langle V, V\rangle=\|V\|_{2}^{2} .
$$


This yields at once,

$$
\mathrm{I}-\mathrm{IV}=0 .
$$

Consider now term II of (4.2.10). By using Lemma 2.1.16, the definition of $\underline{\underline{c}}_{\underline{G}}$ in (3.3.2) and (4.2.8) we have,

$$
\begin{aligned}
& \left\langle\underline{\underline{\mathfrak{C}}}_{\underline{Q}} \underline{\mathfrak{C}}_{\underline{G}}(\underline{H}), \underline{\mathfrak{C}}_{\underline{B}}(V)\right\rangle=\left\langle\underline{\underline{\mathfrak{C}}}_{\underline{G}} \underline{\underline{\mathfrak{C}}}_{\underline{Q}}(\underline{H}), \underline{\mathfrak{C}}_{\underline{B}}(V)\right\rangle=\left\langle\underline{\underline{\mathfrak{C}}}_{\underline{B}}^{*} \underline{\underline{\mathfrak{C}}}_{\underline{G}} \underline{\underline{\mathfrak{C}}}_{\underline{Q}}(\underline{H}), V\right\rangle
\end{aligned}
$$

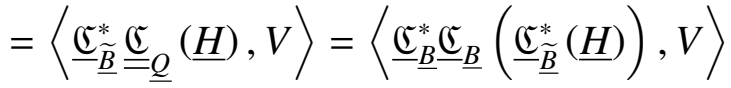

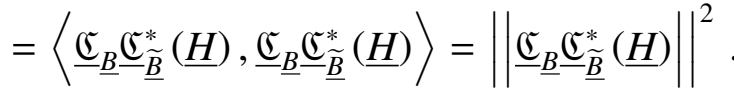

Last, we treat term III of (4.2.10),

$$
\left\langle\underline{H}, \underline{\mathfrak{C}}_{\underline{B}}(V)\right\rangle=\left\langle\underline{H}, \underline{\mathfrak{C}}_{\underline{B}} \underline{\mathfrak{C}}_{\underline{\underline{B}}}^{*} \underline{(H)}\right\rangle .
$$

By strongly admissible and the (NEPC), together with Lemma 4.2.1, we have that

$$
\begin{aligned}
& \left\langle\left(\underline{\underline{\mathfrak{C}}}_{\underline{C}}-\underline{\underline{\mathfrak{E}}}\right)(\underline{H}), \underline{\mathfrak{C}}_{\underline{B}}(V)\right\rangle-\|V\|^{2}=\left\|\underline{\mathfrak{C}}_{\underline{B}} \underline{\mathfrak{C}}_{\underline{\underline{B}}}^{*}(\underline{H})\right\|^{2}-\left\langle\underline{\mathfrak{C}_{\underline{B}}} \underline{\mathfrak{C}}_{\underline{\underline{C}}}^{*}(\underline{H}), \underline{H}\right\rangle
\end{aligned}
$$

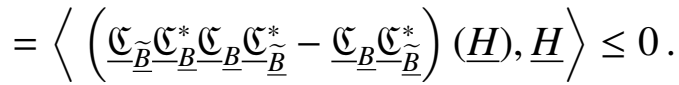

Remark 4.2.5. Notably, in practical applications it seems that weak admissibility suffices for convergence, $c f$. Chapter 5. To extend the following proof of convergence one possible way might be that Lemma 4.2.4 does not need to hold globally for all $(U, W) \in \Gamma_{1+P}$ satisfying (4.2.8).

In the remainder of this chapter let us collect the inequalities that we obtain by our characterization of a fixed point of Algorithm 3.2.8 as a solution of the constrained double minimization Problem 3.3.4.

Let $F \in \mathbb{R}^{n \times m}, \kappa \in\{1,2\}, \beta \in \mathbb{R}_{+}$and let $(A, \underline{B}, \underline{\widetilde{B}})$ be input filters then we have seen that the updating steps of Algorithm (3.2.8) are given by minimizing the following two functionals

$$
\begin{aligned}
& \mathcal{J}_{1}(U, \underline{W}, \underline{\lambda})=|\underline{W}|_{1, \kappa}+\frac{\beta}{2}\left\|\underline{W}-\underline{\mathfrak{C}}_{\underline{B}}(U)\right\|^{2}+\langle\underline{\lambda}, \underline{W}\rangle, \\
& \mathcal{J}_{2}(U, \underline{W}, \underline{\lambda})=\frac{\beta}{2}\left\|\mathfrak{C}_{L}(F)-U\right\|^{2}+\frac{\beta}{2}\left\|\underline{\underline{\mathfrak{C}}}_{\underline{C}}(\underline{W})-\underline{\mathfrak{C}}_{\underline{B}}(U)\right\|^{2}-\left\langle\underline{\underline{\mathfrak{C}}}_{\underline{C}}(\underline{\lambda}), \underline{\mathfrak{C}}_{\underline{B}}(U)\right\rangle,
\end{aligned}
$$

with $\underline{\underline{c}}_{C}$ defined via (3.3.3) and $\mathfrak{c}_{L}$ defined via (3.3.5), see Chapter 3.3. We apply Proposition 2.2.2 to $\mathcal{J}_{1}$ and $\mathcal{J}_{2}$. 
Corollary 4.2.6. Let $F \in \mathbb{R}^{n \times m}, \kappa \in\{1,2\}, \beta \in \mathbb{R}_{+}$and let $(A, \underline{B}, \underline{\widetilde{B}}) \in \Gamma_{1+2 P}$ be input filters, with $(\underline{B}, \underline{\widetilde{B}})$ strongly admissible and satisfy the $(N E P C)$. Let $\mathcal{J}_{1}$ and $\mathcal{J}_{2}$ be defined as in (4.2.12).

(i) For a fixed $U \in \mathbb{R}^{n \times m}$ and $\underline{\lambda} \in \Gamma_{P}$, let $\underline{W}^{\dagger}$ be a minimizer of $\mathcal{J}_{1}$ w.r.t. $\underline{W}$ then

$$
0 \leq \beta\left\langle\underline{W}^{\dagger}-\underline{\mathfrak{c}}_{\underline{B}}(U), \underline{W}-\underline{W}^{\dagger}\right\rangle+|\underline{W}|_{1, \kappa}-\left|\underline{W}^{\dagger}\right|_{1, \kappa}+\left\langle\underline{\lambda}, \underline{W}-\underline{W}^{\dagger}\right\rangle, \quad \forall \underline{W} \in \Gamma_{P} .
$$

(ii) For a fixed $\underline{W} \in \Gamma_{\mathbb{R}}$ and $\underline{\lambda} \in \Gamma_{P}$, let $U^{\dagger}$ be a minimizer of $\mathcal{J}_{2}$ w.r.t. $U$ then

$$
\begin{aligned}
0 \leq & \beta\left\langle\underline{\mathfrak{C}}_{\underline{B}}\left(U^{\dagger}\right)-\underline{\underline{\mathfrak{C}}}_{\underline{C}}(\underline{W}), \underline{\mathfrak{C}}_{\underline{B}}(U)-\underline{\mathfrak{C}}_{\underline{B}}\left(U^{\dagger}\right)\right\rangle+\beta\left\langle U^{\dagger}-\mathfrak{C}_{L}(F), U-U^{\dagger}\right\rangle \\
& -\left\langle\underline{\underline{\mathfrak{C}}}_{\underline{C}}(\underline{\lambda}), \underline{\mathfrak{C}}_{\underline{B}}(U)-\underline{\mathfrak{C}}_{\underline{B}}\left(U^{\dagger}\right)\right\rangle, \quad \forall U \in \mathbb{R}^{n \times m} .
\end{aligned}
$$

Proof. Let us start by remarking, that $\mathcal{J}_{1}$ and $\mathcal{J}_{2}$ are both coercive w.r.t. $\underline{W}$ and $U$, respectively.

(i) For a fixed $U \in \mathbb{R}^{n \times m}$ and $\underline{\lambda} \in \Gamma_{P}$ we define

$$
\mathcal{F}_{1}(\underline{W}):=\frac{\beta}{2} \| \underline{W}-\left.\underline{\mathfrak{C}}_{\underline{B}}(U)\right|^{2}, \quad \mathcal{F}_{2}(\underline{W}):=|\underline{W}|_{1, \kappa}+\langle\underline{\lambda}, \underline{W}\rangle .
$$

Since, $\mathcal{F}_{1}$ is differentiable and convex and $\mathcal{F}_{2}$ is lower-semicontinuous and convex we have by Proposition 2.2.2 that (4.2.13) holds for any minimizer $\underline{W}^{\dagger}$ of $\mathcal{F}_{1}+\mathcal{F}_{2}$.

(ii) For a fixed $\underline{W}, \underline{\lambda} \in \Gamma_{P}$ we define

$$
\begin{aligned}
& \mathcal{F}_{1}(U):=\frac{\beta}{2}\left\|\underline{\underline{\mathfrak{C}}}_{\underline{C}}(\underline{W})-\underline{\mathfrak{C}}_{\underline{B}}(U)\right\|^{2}+\frac{\beta}{2}\left\|\mathfrak{C}_{L}(F)-U\right\|^{2}, \\
& \mathcal{F}_{2}(U):=-\left\langle\underline{\underline{\mathfrak{C}}}_{\underline{C}}(\underline{\lambda}), \underline{\mathfrak{C}}_{\underline{B}}(U)\right\rangle .
\end{aligned}
$$

Since, $\mathcal{F}_{1}$ is differentiable and convex and $\mathcal{F}_{2}$ is lower-semicontinuous and convex we have by Proposition 2.2.2 that (4.2.14) holds for any minimizer $U$ of $\mathcal{F}_{1}+\mathcal{F}_{2}$.

\subsubsection{Proof of Convergence}

Let $F \in \mathbb{R}^{n \times m}, \kappa \in\{1,2\}, \beta \in \mathbb{R}_{+}$, and let $(A, \underline{B}, \underline{\widetilde{B}}) \in \Gamma_{1+2 P}$ be input filters, with $(\underline{B}, \underline{\widetilde{B}})$ strongly admissible and satisfy the (NEPC). Assume there exists a fixed point $\left(U^{\dagger}, \underline{W}^{\dagger}, \underline{\lambda}^{\dagger}\right) \in \Gamma_{1+2 P}$ of Algorithm 3.2.8. We show the convergence of the iteration in Algorithm 3.2.8 in two steps: 
1. First, in the technical Lemma 4.2.7 we show that the norms of $\underline{\lambda}^{(\tau)}-\lambda^{\dagger}$ and $\underline{\mathfrak{c}}_{\underline{B}}\left(U^{(\tau)}\right)-\underline{\mathfrak{c}}_{\underline{B}}\left(U^{\dagger}\right)$ are bounded for $\tau \rightarrow \infty$.

2. Second, in Theorem 4.2.8 we infer convergence.

These results are derived using the proof techniques of Theorem 3.3 and Theorem 3.4 in $\mathrm{Wu}$ and Tai, see [WT10].

Lemma 4.2.7. Let $F \in \mathbb{R}^{n \times m}, \kappa \in\{1,2\}, \beta \in \mathbb{R}_{+}$and let $(A, \underline{B}, \underline{\widetilde{B}}) \in \Gamma_{1+2 P}$ be input filters, with $(\underline{B}, \underline{\widetilde{B}})$ strongly admissible and satisfy the $(N E P C)$. Assume there exists a fixed point $\left(U^{\dagger}, \underline{W}^{\dagger}, \underline{\lambda}^{\dagger}\right)$ of Algorithm 3.2.8. Consider the iterative procedure of Algorithm 3.2.8 and let $\left\{\left(U^{(\tau)}, \underline{W}^{(\tau)}, \underline{\lambda}^{(\tau)}\right)\right\}_{\tau \in \mathbb{N}}$ be a sequence of updates of Algorithm 3.2.8. Define

$$
\bar{U}^{(\tau)}=U^{(\tau)}-U^{\dagger}, \quad \underline{W}^{(\tau)}=\underline{W}^{(\tau)}-\underline{W}^{\dagger}, \quad \underline{\bar{\lambda}}^{(\tau)}=\underline{\lambda}^{(\tau)}-\underline{\lambda}^{\dagger},
$$

for all $\tau \in \mathbb{N}$. Then,

(i) the sequences

$$
\left(\left\|\underline{\bar{\lambda}}^{(\tau)}\right\|^{2}\right)_{\tau \in \mathbb{N}} \text { and }\left(\left\|\underline{\underline{C}}_{\underline{B}}\left(\bar{U}^{(\tau)}\right)\right\|^{2}\right)_{\tau \in \mathbb{N}},
$$

are bounded.

(ii) In particular, we have

$$
\begin{aligned}
& \left\|\underline{\bar{\lambda}}^{(\tau)}\right\|^{2}+\beta^{2}\left\|\underline{\mathfrak{C}}_{\underline{B}}\left(\bar{U}^{(\tau-1)}\right)\right\|^{2}-\left(\left\|\overline{\bar{\lambda}}^{(\tau+1)}\right\|^{2}+\beta^{2}\left\|\underline{\underline{\mathfrak{C}}}_{\underline{B}}\left(\bar{U}^{(\tau)}\right)\right\|^{2}\right) \\
& \geq \beta^{2}\left\|\underline{\bar{W}}^{(\tau)}-\underline{\mathfrak{C}}_{\underline{B}}\left(\bar{U}^{(\tau)}\right)\right\|^{2}+\beta^{2}\left\|\underline{\mathfrak{C}}_{\underline{B}}\left(\bar{U}^{(\tau)}-\bar{U}^{(\tau-1)}\right)\right\|^{2},
\end{aligned}
$$

for all $\tau \in \mathbb{N}_{\geq 2}$.

(iii) If there exists $\underline{W} \in \Gamma_{P}$ such that

$$
U^{(0)}=\mathfrak{C}_{A}(F)+\underline{\mathfrak{C}}_{\underline{\underline{B}}}^{*}\left(\underline{W}+\frac{1}{\beta} \underline{\underline{\lambda}}^{(1)}\right)
$$

then (4.2.17) holds also for $\tau=1$.

Proof. The proof techniques of this proof are by Wu and Tai, taken from Theorem 4.3 and Theorem 4.4 in [WT10].

The main task of the proof is to bound

$$
T:=\left\|\underline{\bar{\lambda}}^{(\tau)}\right\|^{2}+\beta^{2}\left\|\underline{\mathfrak{C}}_{\underline{B}}\left(\bar{U}^{(\tau-1)}\right)\right\|^{2}-\left(\left\|\underline{\bar{\lambda}}^{(\tau+1)}\right\|^{2}+\beta^{2}\left\|\underline{\mathfrak{C}}_{\underline{B}}\left(\bar{U}^{(\tau)}\right)\right\|^{2}\right),
$$


for $\tau \in \mathbb{N}_{\geq 2}$ from below by positive terms.

First note that since $\left(U^{\dagger}, \underline{W}^{\dagger}, \underline{\lambda}^{\dagger}\right)$ is a fixed point we have that $\underline{\mathfrak{C}}_{\underline{B}}\left(U^{\dagger}\right)=\underline{W}^{\dagger}$. Together with the definition of an update $\underline{\lambda}^{(\tau+1)}$ in Algorithm (3.2.8) we obtain

$$
\underline{\bar{\lambda}}^{(\tau+1)}=\underline{\lambda}^{(\tau)}-\underline{\lambda}^{\dagger}+\beta\left(\underline{W}^{(\tau)}-\underline{\mathfrak{C}}_{\underline{B}}\left(U^{(\tau)}\right)\right)=\underline{\bar{\lambda}}^{(\tau)}+\beta\left(\underline{\bar{W}}^{(\tau)}-\underline{\mathfrak{C}}_{\underline{B}}\left(\bar{U}^{(\tau)}\right)\right) .
$$

This yields,

$$
\left\|\underline{\bar{\lambda}}^{(\tau)}\right\|^{2}-\left\|\underline{\bar{\lambda}}^{(\tau+1)}\right\|^{2}=-2 \beta\left\langle\underline{\bar{\lambda}}^{(\tau)}, \underline{\bar{W}}^{(\tau)}-\underline{\mathfrak{c}}_{\underline{B}}\left(\bar{U}^{(\tau)}\right)\right\rangle-\beta^{2}\left\|\underline{\bar{W}}^{(\tau)}-\underline{\mathfrak{c}}_{\underline{B}}\left(\bar{U}^{(\tau)}\right)\right\|^{2},
$$

for all $\tau \in \mathbb{N}$.

In order to bound the right-hand side of (4.2.20) from below, we need to use Corollary 4.2.6 in various situations.

(i) First, we use that $\underline{W}^{\dagger}$ minimizes $\mathcal{J}_{1}$ given in (4.2.12) for fixed $U^{\dagger}$ and $\underline{\lambda}^{\dagger}$, by $\left(U^{\dagger}, \underline{W}^{\dagger}, \underline{\lambda}^{\dagger}\right)$ being a fixed point of Algorithm 3.2.8. By (i) of Corollary 4.2.6 we have

$$
\begin{aligned}
0 \leq \beta & \left\langle\underline{W}^{\dagger}-\underline{\underline{C}}_{\underline{B}}\left(U^{\dagger}\right), \underline{W}-\underline{W}^{\dagger}\right\rangle+|\underline{W}|_{1, \kappa}-\left|\underline{W}^{\dagger}\right|_{1, \kappa} \\
& +\left\langle\underline{\lambda^{\dagger}}, \underline{W}-\underline{W^{\dagger}}\right\rangle, \quad \forall \underline{W} \in \Gamma_{P} .
\end{aligned}
$$

(ii) Second, we use that $\underline{W}^{(\tau)}$ minimizes $\mathcal{J}_{1}$ given in (4.2.12) for fixed $U^{(\tau-1)}$ and $\underline{\lambda}^{(\tau)}$, by the updating step of Algorithm (3.2.8). By (i) of Corollary 4.2.6 we have

$$
\begin{aligned}
0 \leq \beta & \left\langle\underline{W}^{(\tau)}-\underline{\mathfrak{C}}_{\underline{B}}\left(U^{(\tau-1)}\right), \underline{W}-\underline{W}^{(\tau)}\right\rangle+|\underline{W}|_{1, \kappa}-\left.|| \underline{W}^{(\tau)}\right|_{1, \kappa} \\
& +\left\langle\underline{\lambda}^{(\tau)}, \underline{W}-\underline{W}^{(\tau)}\right\rangle, \quad \forall \underline{W} \in \Gamma_{P},
\end{aligned}
$$

for all $\tau \in \mathbb{N}$.

(iii) Third, we use that $U^{\dagger}$ minimizes $\mathcal{J}_{2}$ given in (4.2.12) for fixed $\underline{W}^{\dagger}$ and $\underline{\lambda}^{\dagger}$, by $\left(U^{\dagger}, \underline{W}^{\dagger}, \underline{\lambda}^{\dagger}\right)$ being a fixed point of Algorithm 3.2.8. By (ii) of Corollary 4.2.6 we have

$$
\begin{aligned}
0 \leq \beta & \left\langle\underline{\mathfrak{C}}_{\underline{B}}\left(U^{\dagger}\right)-\underline{\underline{\mathfrak{C}}}_{\underline{C}}\left(\underline{W}^{\dagger}\right), \underline{\mathfrak{C}}_{\underline{B}}(U)-\underline{\mathfrak{C}}_{\underline{B}}\left(U^{\dagger}\right)\right\rangle+\beta\left\langle U^{\dagger}-\mathfrak{C}_{L}(F), U-U^{\dagger}\right\rangle \\
& -\left\langle\underline{\underline{\mathfrak{C}}}_{\underline{C}}\left(\underline{\lambda}^{\dagger}\right), \underline{\mathfrak{C}}_{\underline{B}}(U)-\underline{\mathfrak{C}}_{\underline{B}}\left(U^{\dagger}\right)\right\rangle, \quad \forall U \in \mathbb{R}^{n \times m} .
\end{aligned}
$$

(iv) Last, we use that $U^{(\tau)}$ minimizes $\mathcal{J}_{2}$ given in (4.2.12) for fixed $\underline{W}^{(\tau)}$ and $\underline{\lambda}^{(\tau)}$, by 
the updating step of Algorithm (3.2.8). By (ii) of Corollary 4.2.6 we have

$$
\begin{aligned}
0 \leq \beta & \left\langle\underline{\underline{\mathfrak{C}}}_{\underline{B}}\left(U^{(\tau)}\right)-\underline{\underline{\mathfrak{C}}}_{\underline{C}}\left(\underline{W}^{(\tau)}\right), \underline{\mathfrak{C}}_{\underline{B}}(U)-\underline{\mathfrak{C}}_{\underline{B}}\left(U^{(\tau)}\right)\right\rangle+\beta\left\langle U^{(\tau)}-\mathfrak{C}_{L}(F), U-U^{(\tau)}\right\rangle \\
& -\left\langle\underline{\underline{\mathfrak{C}}}_{\underline{C}}\left(\underline{\lambda}^{(\tau)}\right), \underline{\mathfrak{C}}_{\underline{B}}(U)-\underline{\mathfrak{C}}_{\underline{B}}\left(U^{(\tau)}\right)\right\rangle, \quad \forall U \in \mathbb{R}^{n \times m},
\end{aligned}
$$

for all $\tau \in \mathbb{N}$.

Plugging in $\underline{W}^{(\tau)}$ for $\underline{W}$ in (4.2.21) and $\underline{W}^{\dagger}$ for $\underline{W}$ in (4.2.22) and adding them up yields,

$$
0 \leq \beta\left\langle\underline{W}^{\dagger}-\underline{W}^{(\tau)}-\underline{\mathfrak{C}}_{\underline{B}}\left(U^{\dagger}-U^{(\tau-1)}\right), \underline{W}^{(\tau)}\right\rangle+\left\langle\underline{\lambda}^{\dagger}-\underline{\lambda}^{(\tau)}, \underline{W}^{(\tau)}\right\rangle
$$

Yielding, via simplification and multiplication with -1 the inequality

$$
0 \geq \beta\left\langle\underline{\underline{W}}^{(\tau)}-\underline{\mathfrak{C}}_{\underline{B}}\left(\bar{U}^{(\tau-1)}\right), \underline{\bar{W}}^{(\tau)}\right\rangle+\left\langle\underline{\bar{\lambda}}^{(\tau)}, \underline{\bar{W}}^{(\tau)}\right\rangle .
$$

Plugging in $U^{(\tau)}$ for $U$ in (4.2.23) and $U^{\dagger}$ for $U$ in (4.2.24) and adding them up we obtain

$$
\begin{aligned}
0 \leq \beta & \left\langle\underline{\mathfrak{C}}_{\underline{B}}\left(U^{\dagger}-U^{(\tau)}\right)-\underline{\underline{\mathfrak{C}}}_{\underline{C}}\left(\underline{W}^{\dagger}-\underline{W}^{(\tau)}\right), \underline{\mathfrak{C}}_{\underline{B}}\left(\bar{U}^{(\tau)}\right)\right\rangle-\left\langle\underline{\underline{\mathfrak{C}}}_{\underline{C}}\left(\underline{\lambda}^{\dagger}-\underline{\lambda}^{(\tau)}\right), \underline{\mathfrak{C}}_{\underline{B}}\left(\bar{U}^{(\tau)}\right)\right\rangle \\
& +\beta\left\langle-\mathfrak{C}_{L}(F)+U^{\dagger}-U^{(\tau)}+\mathfrak{\mathfrak { C }}_{L}(F), \bar{U}^{(\tau)}\right\rangle .
\end{aligned}
$$

We obtain via simplification and multiplication with -1 the inequality

$$
0 \geq-\beta\left\langle\underline{\underline{\mathfrak{c}}}_{\underline{C}}\left(\underline{\bar{W}}^{(\tau)}\right)-\underline{\mathfrak{c}}_{\underline{B}}\left(\bar{U}^{(\tau)}\right), \underline{\mathfrak{C}}_{\underline{B}}\left(\bar{U}^{(\tau)}\right)\right\rangle-\left\langle\underline{\underline{\mathfrak{c}}}_{\underline{C}}\left(\underline{\bar{\lambda}}^{(\tau)}\right), \underline{\mathfrak{c}}_{\underline{B}}\left(\bar{U}^{(\tau)}\right)\right\rangle+\beta\left\|\bar{U}^{(\tau)}\right\|^{2} .
$$

Let us expand this inequality by adding 0 on the right hand side

$$
\begin{aligned}
0 \geq- & \beta\left\langle\underline{\underline{W}}^{(\tau)}-\underline{\mathfrak{C}}_{\underline{B}}\left(\bar{U}^{(\tau)}\right), \underline{\mathfrak{C}}_{\underline{B}}\left(\bar{U}^{(\tau)}\right)\right\rangle-\left\langle\underline{\bar{\lambda}}^{(\tau)}, \underline{\mathfrak{C}}_{\underline{B}}\left(\bar{U}^{(\tau)}\right)\right\rangle \\
& -\beta\left\langle\underline{\underline{\mathfrak{C}}}_{\underline{C}}\left(\overline{\underline{W}}^{(\tau)}+\frac{1}{\beta} \underline{\bar{\lambda}}^{(\tau)}\right)-\left(\underline{\underline{W}}^{(\tau)}+\frac{1}{\beta} \overline{\bar{\lambda}}^{(\tau)}\right), \underline{\mathfrak{C}}_{\underline{B}}\left(\bar{U}^{(\tau)}\right)\right\rangle+\beta\left\|\bar{U}^{(\tau)}\right\|^{2} .
\end{aligned}
$$

Since,

$$
\begin{aligned}
\underline{\mathfrak{C}}_{\underline{\widetilde{B}}}^{*}\left(\underline{\bar{W}}^{(\tau)}+\frac{1}{\beta} \underline{\bar{\lambda}}^{(\tau)}\right) & =\underline{\mathfrak{C}}_{\underline{\underline{B}}}^{*}\left(\underline{W}^{(\tau)}+\frac{1}{\beta} \underline{\lambda}^{(\tau)}\right)-\underline{\mathfrak{C}}_{\underline{\underline{B}}}^{*}\left(\underline{W}^{\dagger}+\frac{1}{\beta} \underline{\lambda}^{\dagger}\right)+\mathfrak{C}_{A}(F)-\mathfrak{C}_{A}(F) \\
& =U^{(\tau)}-U^{\dagger}=\bar{U}^{(\tau)},
\end{aligned}
$$

by strongly admissible and satisfy the (NEPC) of $(\underline{B}, \underline{\widetilde{B}})$ we can apply Lemma 4.2 .4 for 
$H:=\underline{W}^{(\tau)}+1 / \beta \underline{\bar{\lambda}}^{(\tau)}$ and $V:=\bar{U}^{(\tau)}$ to obtain

$$
0 \geq-\beta\left\langle\underline{\underline{W}}^{(\tau)}-\underline{\mathfrak{C}}_{\underline{B}}\left(\bar{U}^{(\tau)}\right), \underline{\mathfrak{C}}_{\underline{B}}\left(\bar{U}^{(\tau)}\right)\right\rangle-\left\langle\underline{\bar{\lambda}}^{(\tau)}, \underline{\mathfrak{C}}_{\underline{B}}\left(\bar{U}^{(\tau)}\right)\right\rangle .
$$

Adding (4.2.25), (4.2.26) and a zero-term we obtain

$$
\begin{aligned}
0 \geq & \beta\left\langle\bar{W}^{(\tau)}, \bar{W}^{(\tau)}-\underline{\mathfrak{c}}_{\underline{B}}\left(\bar{U}^{(\tau)}\right)\right\rangle-\beta\left\langle\underline{\mathfrak{c}}_{\underline{B}}\left(\bar{U}^{(\tau-1)}\right), \underline{W}^{(\tau)}\right\rangle+\beta\left\langle\underline{\mathfrak{c}}_{\underline{B}}\left(\bar{U}^{(\tau)}\right), \underline{\mathfrak{c}}_{\underline{B}}\left(\bar{U}^{(\tau)}\right)\right\rangle \\
& +\left\langle\underline{\bar{\lambda}}^{(\tau)}, \bar{W}^{(\tau)}-\underline{\mathfrak{c}}_{\underline{B}}\left(\bar{U}^{(\tau)}\right)\right\rangle \\
= & \beta\left\langle\bar{W}^{(\tau)}-\underline{\mathfrak{C}}_{\underline{B}}\left(\bar{U}^{(\tau)}\right), \underline{W}^{(\tau)}-\underline{\mathfrak{c}}_{\underline{B}}\left(\bar{U}^{(\tau)}\right)\right\rangle+\left\langle\underline{\bar{\lambda}}^{(\tau)}, \underline{\bar{W}}^{(\tau)}-\underline{\mathfrak{c}}_{\underline{B}}\left(\bar{U}^{(\tau)}\right)\right\rangle \\
& +\beta\left\langle\underline{\mathfrak{c}}_{\underline{B}}\left(\bar{U}^{(\tau)}\right)-\underline{\mathfrak{c}}_{\underline{B}}\left(\bar{U}^{(\tau-1)}\right), \bar{W}^{(\tau)}\right\rangle,
\end{aligned}
$$

multiplying with $2 \beta$ and simplifying yields

$$
\begin{aligned}
-2 \beta & \left\langle\underline{\bar{\lambda}}^{(\tau)}, \underline{\bar{W}}^{(\tau)}-\underline{\mathfrak{c}}_{\underline{B}}\left(\bar{U}^{(\tau)}\right)\right\rangle-\beta^{2}\left\|\underline{\underline{W}}^{(\tau)}-\underline{\mathfrak{c}}_{\underline{B}}\left(\bar{U}^{(\tau)}\right)\right\|^{2} \\
& \geq \beta^{2}\left\|\underline{\underline{W}}^{(\tau)}-\underline{\mathfrak{c}}_{\underline{B}}\left(\bar{U}^{(\tau)}\right)\right\|^{2}+2 \beta^{2}\left\langle\underline{\mathfrak{c}}_{\underline{B}}\left(\bar{U}^{(\tau)}-\bar{U}^{(\tau-1)}\right), \underline{W}^{(\tau)}\right\rangle .
\end{aligned}
$$

We have achieved a first step: The right-hand side of (4.2.20) can be found on the left-hand-side of (4.2.27) and it is bounded from below by a quadratic term and a linear one. Now we need to deal with the linear term on the right-hand side of (4.2.27). Let from now on $\tau \in \mathbb{N}_{\geq 2}$ or assume for $\tau=1$ the existence of some $\underline{W}^{(0)} \in \Gamma_{P}$ such that

$$
U^{(0)}=\mathfrak{C}_{A}(F)+\underline{\mathbb{C}}_{\widetilde{B}}^{*}\left(\underline{W}+\frac{1}{\beta} \underline{\lambda}^{(1)}\right)
$$

Split the linear term on the r.h.s. of (4.2.27) into three parts by adding 0 terms,

$$
\begin{aligned}
\left\langle\underline{\mathfrak{C}}_{\underline{B}}\left(\bar{U}^{(\tau)}-\bar{U}^{(\tau-1)}\right), \underline{\bar{W}}^{(\tau)}\right\rangle= & \underbrace{\left\langle\underline{\mathfrak{c}}_{\underline{B}}\left(\bar{U}^{(\tau)}-\bar{U}^{(\tau-1)}\right), \underline{W}^{(\tau)}-\underline{W}^{(\tau-1)}\right\rangle}_{\text {I }} \\
& +\underbrace{\left\langle\underline{\mathfrak{C}}_{\underline{B}}\left(\bar{U}^{(\tau)}-\bar{U}^{(\tau-1)}\right), \bar{W}^{(\tau-1)}-\mathfrak{C}_{B}\left(\bar{U}^{(\tau-1)}\right)\right\rangle}_{\text {II }} \\
& +\underbrace{\left\langle\underline{\mathfrak{C}}_{\underline{B}}\left(\bar{U}^{(\tau)}-\bar{U}^{(\tau-1)}\right), \underline{\mathfrak{C}}_{\underline{B}}\left(\bar{U}^{(\tau-1)}\right)\right\rangle}_{\text {III }} .
\end{aligned}
$$

We start by investigating I and II of (4.2.28). To this end plug in $U^{(\tau-1)}$ for $U$ in (4.2.24) 
to obtain

$$
\begin{aligned}
0 \leq \beta & \left\langle\underline{\mathfrak{C}}_{\underline{B}}\left(U^{(\tau)}\right)-\underline{\underline{\mathfrak{C}}}_{\underline{C}}\left(\underline{W}^{(\tau)}\right), \underline{\mathfrak{C}}_{\underline{B}}\left(U^{(\tau-1)}\right)-\underline{\mathfrak{C}}_{\underline{B}}\left(U^{(\tau)}\right)\right\rangle \\
& +\beta\left\langle U^{(\tau)}-\mathfrak{\mathfrak { C }}_{L}(F), U^{(\tau-1)}-U^{(\tau)}\right\rangle-\left\langle\underline{\underline{\mathfrak{C}}}_{\underline{C}}\left(\underline{\lambda}^{(\tau)}\right), \underline{\mathfrak{C}}_{\underline{B}}\left(U^{(\tau-1)}\right)-\underline{\mathfrak{C}}_{\underline{B}}\left(U^{(\tau)}\right)\right\rangle,
\end{aligned}
$$

and plug in $(\tau-1)$ for $(\tau)$ and $U^{(\tau)}$ for $U$ in (4.2.24),

$$
\begin{aligned}
0 \leq \beta & \left\langle\underline{\mathfrak{C}}_{\underline{B}}\left(U^{(\tau-1)}\right)-\underline{\underline{\mathfrak{C}}}_{\underline{C}}\left(\underline{W}^{(\tau-1)}\right), \underline{\mathfrak{C}}_{\underline{B}}\left(U^{(\tau)}\right)-\underline{\mathfrak{C}}_{\underline{B}}\left(U^{(\tau-1)}\right)\right\rangle \\
& +\beta\left\langle U^{(\tau-1)}-\mathfrak{\mathfrak { C }}_{L}(F), U^{(\tau)}-U^{(\tau-1)}\right\rangle-\left\langle\underline{\underline{\mathfrak{C}}}_{\underline{C}}\left(\underline{\lambda}^{(\tau-1)}\right), \underline{\mathfrak{C}}_{\underline{B}}\left(U^{(\tau)}\right)-\underline{\mathfrak{C}}_{\underline{B}}\left(U^{(\tau-1)}\right)\right\rangle .
\end{aligned}
$$

Adding (4.2.29) and (4.2.30) yields,

$$
\begin{aligned}
0 \leq & \beta\left\langle\underline{\underline{\mathfrak{C}}}_{\underline{C}}\left(\underline{W}^{(\tau)}-\underline{W}^{(\tau-1)}\right)+\underline{\mathfrak{C}}_{\underline{B}}\left(U^{(\tau-1)}-U^{(\tau)}\right), \underline{\mathfrak{C}}_{\underline{B}}\left(U^{(\tau)}-U^{(\tau-1)}\right)\right\rangle \\
& +\beta\left\langle U^{(\tau-1)}-U^{(\tau)}, U^{(\tau)}-U^{(\tau-1)}\right\rangle-\left\langle\underline{\underline{\mathfrak{C}}}_{\underline{C}}\left(\underline{\lambda}^{(\tau-1)}-\underline{\lambda}^{(\tau)}\right), \underline{\mathfrak{C}}_{\underline{B}}\left(U^{(\tau)}-U^{(\tau-1)}\right)\right\rangle \\
= & \beta\left\langle\underline{\underline{\mathfrak{C}}}_{\underline{C}}\left(\underline{W}^{(\tau)}-\underline{W}^{(\tau-1)}+\frac{1}{\beta}\left(\underline{\lambda}^{(\tau)}-\underline{\lambda}^{(\tau-1)}\right)\right), \underline{\mathfrak{C}}_{\underline{B}}\left(U^{(\tau)}-U^{(\tau-1)}\right)\right\rangle \\
& -\beta\left\|U^{(\tau)}-U^{(\tau-1)}, U^{(\tau)}-U^{(\tau-1)}\right\|^{2}-\left\|\underline{\mathfrak{C}}_{\underline{B}}\left(U^{(\tau)}-U^{(\tau-1)}\right)\right\|^{2} .
\end{aligned}
$$

Since,

$$
\begin{aligned}
& \underline{\mathfrak{C}}_{\underline{\underline{B}}}^{*}\left(\underline{W}^{(\tau)}-\underline{W}^{(\tau-1)}+\frac{1}{\beta}\left(\underline{\lambda}^{(\tau)}-\underline{\lambda}^{(\tau-1)}\right)\right) \\
& \quad=\underline{\mathfrak{C}}_{\underline{\widetilde{B}}}^{*}\left(\underline{W}^{(\tau)}+\frac{1}{\beta} \underline{\lambda}^{(\tau)}\right)-\underline{\mathfrak{C}}_{\underline{\widetilde{B}}}^{*}\left(\underline{W}^{(\tau-1)}+\frac{1}{\beta} \underline{\lambda}^{(\tau-1)}\right)+\mathfrak{C}_{A}(F)-\mathfrak{C}_{A}(F) \\
& \quad=U^{(\tau)}-U^{(\tau-1)},
\end{aligned}
$$

for all $\tau \in \mathbb{N}$, we can by strongly admissible and satisfy the (NEPC) of $(\underline{B}, \underline{\widetilde{B}})$ apply Lemma 4.2.4 for $H:=\underline{W}^{(\tau)}-\underline{W}^{(\tau-1)}+1 / \beta\left(\underline{\lambda}^{(\tau)}-\underline{\lambda}^{(\tau-1)}\right)$ and $V:=U^{(\tau)}-U^{(\tau-1)}$ in $(4.2 .31)$ yielding

$$
\begin{aligned}
0 \leq & \beta\left\langle\underline{W}^{(\tau)}-\underline{W}^{(\tau-1)}+\frac{1}{\beta}\left(\underline{\lambda}^{(\tau)}-\underline{\lambda}^{(\tau-1)}\right), \underline{\mathfrak{C}}_{\underline{B}}\left(U^{(\tau)}-U^{(\tau-1)}\right)\right\rangle \\
& -\left\|\underline{\mathfrak{C}}_{\underline{B}}\left(U^{(\tau)}-U^{(\tau-1)}\right)\right\|^{2} .
\end{aligned}
$$


Since,

$$
\begin{aligned}
U^{(\tau)}-U^{(\tau-1)} & =\bar{U}^{(\tau)}-\bar{U}^{(\tau-1)}, \\
\underline{W}^{(\tau)}-\underline{W}^{(\tau-1)} & =\bar{W}^{(\tau)}-\bar{W}^{(\tau-1)}, \\
\underline{\lambda}^{(\tau)}-\underline{\lambda}^{(\tau-1)} & =\underline{\bar{\lambda}}^{(\tau)}-\underline{\bar{\lambda}}^{(\tau-1)},
\end{aligned}
$$

we obtain,

$$
\begin{aligned}
0 \leq & \beta\left\langle\underline{\bar{W}}^{(\tau)}-\underline{\bar{W}}^{(\tau-1)}+\frac{1}{\beta}\left(\underline{\bar{\lambda}}^{(\tau)}-\underline{\bar{\lambda}}^{(\tau-1)}\right), \underline{\mathfrak{C}}_{\underline{B}}\left(\bar{U}^{(\tau)}-\bar{U}^{(\tau-1)}\right)\right\rangle \\
& -\left\|\underline{\mathfrak{C}}_{\underline{B}}\left(\bar{U}^{(\tau)}-\bar{U}^{(\tau-1)}\right)\right\|^{2} .
\end{aligned}
$$

We multiply (4.2.32) with $1 / \beta$ and rework using (4.2.19):

$$
\begin{aligned}
& 0 \leq\left\langle\underline{\underline{W}}^{(\tau)}-\underline{\underline{W}}^{(\tau-1)}+\frac{1}{\beta}\left(\underline{\bar{\lambda}}^{(\tau)}-\underline{\bar{\lambda}}^{(\tau-1)}\right), \underline{\mathfrak{C}}_{\underline{B}}\left(\bar{U}^{(\tau)}-\bar{U}^{(\tau-1)}\right)\right\rangle-\left\|\underline{\mathfrak{C}}_{\underline{B}}\left(\bar{U}^{(\tau)}-\bar{U}^{(\tau-1)}\right)\right\|^{2} \\
& =\left\langle\underline{\bar{W}}^{(\tau)}-\underline{\bar{W}}^{(\tau-1)}+\underline{\bar{W}}^{(\tau-1)}-\underline{\mathfrak{c}}_{\underline{B}}\left(\bar{U}^{(\tau-1)}\right), \underline{\mathfrak{c}}_{\underline{B}}\left(\bar{U}^{(\tau)}-\bar{U}^{(\tau-1)}\right)\right\rangle-\left\|\underline{\mathfrak{c}}_{\underline{B}}\left(\bar{U}^{(\tau)}-\bar{U}^{(\tau-1)}\right)\right\|^{2} \\
& =\underbrace{\left\langle\underline{\underline{W}}^{(\tau)}-\underline{\bar{W}}^{(\tau-1)}, \underline{\mathfrak{C}}_{\underline{B}}\left(\bar{U}^{(\tau)}-\bar{U}^{(\tau-1)}\right)\right\rangle}_{\text {I }}+\underbrace{\left\langle\underline{\underline{W}}^{(\tau-1)}-\underline{\mathfrak{C}}_{\underline{B}}\left(\bar{U}^{(\tau-1)}\right), \underline{\mathfrak{C}}_{\underline{B}}\left(\bar{U}^{(\tau)}-\bar{U}^{(\tau-1)}\right)\right\rangle}_{\text {II }} \\
& -\left\|\underline{\mathfrak{C}_{B}}\left(\bar{U}^{(\tau)}-\bar{U}^{(\tau-1)}\right)\right\|^{2},
\end{aligned}
$$

where I and II coincide with those defined in (4.2.28). Hence, we obtain for I and II of (4.2.28) the relation

$$
\mathrm{I}+\mathrm{II} \geq\left\|\underline{\mathfrak{c}}_{\underline{B}}\left(\bar{U}^{(\tau)}-\bar{U}^{(\tau-1)}\right)\right\|^{2} .
$$

It remains to treat term III of (4.2.28):

$$
\begin{aligned}
& \left\langle\underline{\mathfrak{C}}_{\underline{B}}\left(\bar{U}^{(\tau)}-\bar{U}^{(\tau-1)}\right), \underline{\mathfrak{C}}_{\underline{B}}\left(\bar{U}^{(\tau-1)}\right)\right\rangle \\
& =-\left\langle\underline{\mathfrak{C}}_{\underline{B}}\left(\bar{U}^{(\tau)}-\bar{U}^{(\tau-1)}\right), \frac{1}{2}\left(\underline{\mathfrak{C}}_{\underline{B}}\left(\bar{U}^{(\tau)}-\bar{U}^{(\tau-1)}\right)-\underline{\mathfrak{C}}_{\underline{B}}\left(\bar{U}^{(\tau)}+\bar{U}^{(\tau-1)}\right)\right)\right\rangle \\
& =-\frac{1}{2}\left(\left\|\underline{\mathfrak{C}}_{\underline{B}}\left(\bar{U}^{(\tau)}-\bar{U}^{(\tau-1)}\right)\right\|^{2}-\left\|\underline{\mathfrak{C}}_{\underline{B}}\left(\bar{U}^{(\tau)}\right)\right\|^{2}+\left\|\underline{\mathfrak{C}}_{\underline{B}}\left(\bar{U}^{(\tau-1)}\right)\right\|^{2}\right) .
\end{aligned}
$$

Plug (4.2.33) and (4.2.34) into (4.2.28) and we obtain

$$
\begin{aligned}
\left\langle\underline{\mathfrak{C}}_{\underline{B}}\left(\bar{U}^{(\tau)}-\bar{U}^{(\tau-1)}\right), \underline{\bar{W}}^{(\tau)}\right\rangle \\
\quad \geq \frac{1}{2}\left(\left\|\underline{\mathfrak{C}}_{\underline{B}}\left(\bar{U}^{(\tau)}\right)\right\|^{2}-\left\|\underline{\mathfrak{C}}_{\underline{B}}\left(\bar{U}^{(\tau-1)}\right)\right\|^{2}+\left\|\mid \underline{\mathfrak{C}}_{\underline{B}}\left(\bar{U}^{(\tau)}-\bar{U}^{(\tau-1)}\right)\right\|^{2}\right) .
\end{aligned}
$$


Now we can consider $T$ of (4.2.18). Let us start by using (4.2.20) to obtain

$$
\begin{aligned}
T= & \left.\left\|\underline{\bar{\lambda}}^{(\tau)}\right\|\right|^{2}+\beta^{2}\left\|\underline{\mathfrak{C}}_{\underline{B}}\left(\bar{U}^{(\tau-1)}\right)\right\|^{2}-\left(\left.\left\|\overline{\bar{\lambda}}^{(\tau+1)}\right\|\right|^{2}+\beta^{2}\left\|\underline{\mathfrak{C}}_{\underline{B}}\left(\bar{U}^{(\tau)}\right)\right\|^{2}\right) \\
= & -2 \beta\left\langle\underline{\bar{\lambda}}^{(\tau)}, \underline{W}^{(\tau)}-\underline{\mathfrak{C}}_{\underline{B}}\left(\bar{U}^{(\tau)}\right)\right\rangle-\beta^{2}\left\|\underline{\underline{W}}^{(\tau)}-\underline{\mathfrak{C}}_{\underline{B}}\left(\bar{U}^{(\tau)}\right)\right\|^{2} \\
& +\beta^{2}\left(\left\|\underline{\mathfrak{C}}_{\underline{B}}\left(\bar{U}^{(\tau-1)}\right)\right\|^{2}-\left\|\underline{\mathfrak{C}}_{\underline{B}}\left(\bar{U}^{(\tau)}\right)\right\|^{2}\right) .
\end{aligned}
$$

We bound $T$ from below by using (4.2.27),

$$
\begin{aligned}
T & \geq \beta^{2}\left\|\underline{\underline{W}}^{(\tau)}-\underline{\mathfrak{C}}_{\underline{B}}\left(\bar{U}^{(\tau)}\right)\right\|^{2} \\
& +2 \beta^{2}\left(\left\langle\underline{\mathfrak{C}}_{\underline{B}}\left(\bar{U}^{(\tau)}-\bar{U}^{(\tau-1)}\right), \underline{\underline{W}}^{(\tau)}\right\rangle+\frac{1}{2}\left(\left\|\underline{\underline{\mathfrak{C}_{B}}}\left(\bar{U}^{(\tau-1)}\right)\right\|^{2}-\left\|\underline{\mathfrak{C}}_{\underline{B}}\left(\bar{U}^{(\tau)}\right)\right\|^{2}\right)\right) .
\end{aligned}
$$

Finally, plugging in (4.2.35) we end up with

$$
T \geq \beta^{2}\left\|\underline{\bar{W}}^{(\tau)}-\underline{\mathfrak{C}}_{\underline{B}}\left(\bar{U}^{(\tau)}\right)\right\|^{2}+\beta^{2}\left\|\underline{\mathfrak{C}}_{\underline{B}}\left(\bar{U}^{(\tau)}-\bar{U}^{(\tau-1)}\right)\right\|^{2} \geq 0 .
$$

So we have shown assertions (ii) and (iii). Recalling the definition of $T$ in (4.2.18) we derive

$$
\left\|\underline{\bar{\lambda}}^{(\tau)}\right\|^{2}+\beta^{2}\left\|\underline{\mathfrak{C}}_{\underline{B}}\left(\bar{U}^{(\tau-1)}\right)\right\|^{2}-\left(\left\|\overline{\bar{\lambda}}^{(\tau+1)}\right\|^{2}+\beta^{2}\left\|\underline{\mathfrak{C}}_{\underline{B}}\left(\bar{U}^{(\tau)}\right)\right\|^{2}\right) \geq 0
$$

yielding assertion (i).

Theorem 4.2.8. Let $F \in \mathbb{R}^{n \times m}, \kappa \in\{1,2\}, \beta \in \mathbb{R}_{+}$and let $(A, \underline{B}, \underline{\widetilde{B}}) \in \Gamma_{1+2 P}$ be input filters, with $(\underline{B}, \underline{\widetilde{B}})$ strongly admissible and satisfy the (NEPC). Assume Algorithm 3.2.8 has at least one fixed point. Then Algorithm 3.2.8 converges to a fixed point for any starting values $U^{(0)} \in \mathbb{R}^{n \times m}$ and $\underline{\lambda}^{(1)} \in \Gamma_{P}$.

Proof. The proof techniques used in this proof are based on Theorem 3.3 of [WT10] by $\mathrm{Wu}$ and Tai.

By assumption there exists a fixed point $\left(U^{\dagger}, \underline{W^{\dagger}}, \underline{\lambda^{\dagger}}\right)$ of Algorithm 3.2.8. Let us define again

$$
\bar{U}^{(\tau)}=U^{(\tau)}-U^{\dagger}, \quad \underline{\bar{W}}^{(\tau)}=\underline{W}^{(\tau)}-\underline{W}^{\dagger}, \quad \underline{\bar{\lambda}}^{(\tau)}=\underline{\lambda}^{(\tau)}-\underline{\lambda}^{\dagger} .
$$

Since $(\underline{B}, \underline{\widetilde{B}})$ are strongly admissible and satisfy the (NEPC) we can use Lemma 4.2.7 
and conclude that

$$
\left\{\underline{\bar{\lambda}}^{(\tau)}\right\}_{\tau=1}^{\infty} \text { and } \quad\left\{\underline{\mathfrak{C}}_{\underline{B}}\left(\bar{U}^{(\tau-1)}\right)\right\}_{\tau=1}^{\infty}
$$

are bounded. Hence, there exists a converging subsequence $\underline{\lambda}^{\left(\tau_{j}\right)} \stackrel{j \rightarrow \infty}{\longrightarrow} \underline{\lambda}$. Considering the sequence $\left\{\underline{\underline{C}}_{\underline{B}}\left(U^{\left(\tau_{j}-1\right)}\right)\right\}_{j=1}^{\infty}$ is still bounded there exists a converging sub-subsequence $\left\{\tau_{\ell}\right\}_{\ell=1}^{\infty} \subset\left\{\tau_{j}\right\}_{j=1}^{\infty}$. Yielding,

$$
\underline{\lambda}^{\left(\tau_{\ell}\right)} \stackrel{\ell \rightarrow \infty}{\longrightarrow} \underline{\lambda}, \quad \text { and } \quad \underline{\mathfrak{C}}_{\underline{B}}\left(U^{\left(\tau_{\ell}-1\right)}\right) \stackrel{\ell \rightarrow \infty}{\longrightarrow} \underline{\ddot{Z}},
$$

for some $\ddot{\underline{\lambda}}, \underline{\ddot{Z}} \in \Gamma_{P}$.

Since $\underline{W}^{(\tau)}$ depends continuously on $\underline{\mathfrak{C}}_{\underline{B}}\left(U^{(\tau-1)}\right)$ and $\underline{\lambda}^{(\tau)}$, see the updating step of Algorithm 3.2.8, we obtain furthermore

$$
\underline{W}^{\left(\tau_{\ell}\right)} \stackrel{\ell \rightarrow \infty}{\longrightarrow} \underline{\ddot{W}},
$$

for some $\ddot{W} \in \Gamma_{P}$.

The same argument applies for $U^{(\tau)}$, that depends continuously on $\underline{W}^{(\tau)}$ and $\underline{\lambda}^{(\tau)}$, see the updating step of Algorithm 3.2.8, hence,

$$
U^{\left(\tau_{\ell}\right)} \stackrel{\ell \rightarrow \infty}{\longrightarrow} \ddot{U},
$$

for some $\ddot{U} \in \mathbb{R}^{n \times m}$.

By part (ii) of Lemma 4.2.7 we can make repeated use of (4.2.17) to obtain for all $\ell \geq 2$ that

$$
\begin{aligned}
& \sum_{k=\tau_{\ell}}^{\tau_{\ell+1}-1}\left(\beta^{2}\left\|\underline{\underline{W}}^{(k)}-\underline{\mathfrak{C}}_{\underline{B}}\left(\bar{U}^{(k)}\right)\right\|^{2}+\beta^{2}\left\|\underline{\mathfrak{C}}_{\underline{B}}\left(\bar{U}^{(k)}-\bar{U}^{(k-1)}\right)\right\|^{2}\right) \\
& \leq\left\|\underline{\bar{\lambda}}^{\left(\tau_{\ell}\right)}\right\|^{2}+\beta^{2}\left\|\underline{\mathfrak{C}}_{\underline{B}}\left(\bar{U}^{\left(\tau_{\ell-1}-1\right)}\right)\right\|^{2}-\left(\left\|\underline{\bar{\lambda}}^{\left(\tau_{\ell+1}\right)}\right\|^{2}+\beta^{2}\left\|\underline{\mathfrak{C}}_{\underline{B}}\left(\bar{U}^{\left(\tau_{\ell+1}-1\right)}\right)\right\|^{2}\right) \stackrel{\ell \rightarrow \infty}{\longrightarrow} 0,
\end{aligned}
$$

Yielding,

$$
\left\|\underline{\underline{W}}^{(\tau)}-\underline{\mathfrak{c}}_{\underline{B}}\left(\bar{U}^{(\tau)}\right)\right\|^{2} \stackrel{\tau \rightarrow \infty}{\longrightarrow} 0 \quad \text { and } \quad\left\|\underline{\mathfrak{c}}_{\underline{B}}\left(\bar{U}^{(\tau)}-\bar{U}^{(\tau-1)}\right)\right\|^{2} \stackrel{\tau \rightarrow \infty}{\longrightarrow} 0 .
$$

Hence, the point $(\ddot{U}, \underline{\ddot{W}}, \underline{\ddot{\lambda}})$ obeys

$$
\underline{\mathfrak{C}}_{\underline{B}}(\ddot{U})=\underline{\ddot{W}}=\underline{Z} .
$$

By definition of the convergent subsequences in (4.2.39) and (4.2.40) we have that 
$(\ddot{U}, \underline{\ddot{W}}, \ddot{\ddot{\lambda}})$ is a fixed point of Algorithm 3.2.8.

Now exchange the fixed point $\left(U^{\dagger}, \underline{W}^{\dagger}, \underline{\lambda}^{\dagger}\right)$ by our new fixed point $(\ddot{U}, \underline{\ddot{W}}, \underline{\ddot{\lambda}})$ in the notation (4.2.37) to define

$$
\overline{\bar{U}}^{(\tau)}=U^{(\tau)}-\ddot{U}, \quad \underline{\bar{W}}^{(\tau)}=\underline{W}^{(\tau)}-\underline{\ddot{W}}, \quad \underline{\bar{\lambda}}^{(\tau)}=\underline{\lambda}^{(\tau)}-\underline{\ddot{\lambda}},
$$

Then we have by $(4.2 .38)$ that

$$
\underline{\overline{\bar{\lambda}}}^{\left(\tau_{\ell}\right)} \stackrel{\ell \rightarrow \infty}{\longrightarrow} \underline{0}, \quad \text { and } \quad \underline{\mathfrak{C}}_{\underline{B}}\left(\overline{\bar{U}}^{\left(\tau_{\ell}-1\right)}\right) \stackrel{\ell \rightarrow \infty}{\longrightarrow} \underline{0} .
$$

Via (4.2.17) of Lemma 4.2.7[(ii)] this enforces that $\underline{\bar{\lambda}}^{(\tau)}$ and $\left.\underline{\mathfrak{C}}_{\underline{B}} \overline{\bar{U}}^{(\tau)}\right)$ converge to $\underline{0}$ for $\tau \rightarrow \infty$, yielding the convergence of $\underline{\lambda}^{(\tau)}$ and $\underline{\mathfrak{C}}_{\underline{B}}\left(U^{(\tau)}\right)$ to $\underline{\ddot{\lambda}}$ and $\underline{Z}$. The convergence of $U^{(\tau)}$ and $\underline{W}^{(\tau)}$ follows immediately by the argument of (4.2.39) and (4.2.40). Hence,

$$
\left(U^{(\tau)}, \underline{W}^{(\tau)}, \underline{\lambda}^{(\tau)}\right) \stackrel{\tau \rightarrow \infty}{\longrightarrow}(\ddot{U}, \underline{\underline{W}}, \underline{\lambda})
$$

Let us combining the above result with the one of Chapter 4.1 .

Corollary 4.2.9. Let $F \in \mathbb{R}^{n \times m}, \kappa \in\{1,2\}, \beta \in \mathbb{R}_{+}$and let $(A, \underline{B}, \underline{\widetilde{B}}) \in \Gamma_{1+2 P}$ be input filters, with $(\underline{B}, \underline{\widetilde{B}})$ strongly admissible and satisfying the (CPC). Algorithm 3.2.8 converges to a fixed point.

Proof. By weak admissibility and the (CPC) of $(\underline{B}, \underline{\widetilde{B}})$ there exists a fixed point of Algorithm 3.2.8 by Theorem 4.1.7. By using strong admissibility and the now given existence of a fixed point we have by Theorem 4.2.8 that Algorithm 3.2.8 converges to a fixed point.

\subsection{Uniqueness of the Fixed Point}

Now that we have considered the existence of a fixed point of Algorithm 3.2.8 and the convergence to a fixed point, the question whether a given fixed point is unique, arises. Note that when talking about uniqueness we will be focussing on the uniqueness of the resulting cartoon $U$ and not on the auxiliary variables $\underline{W}$ and $\underline{\lambda}$.

Remark 4.3.1. As discussed in Chapter 4.2.1 and Remark 4.2.3, in the case that strong admissibility and the $(C P C)$ hold for $(\underline{B}, \underline{\widetilde{B}})$, we minimize a strictly convex functional. Hence, uniqueness of a fixed point, i.e. minimizer, is given. However, for extendibility to weaker assumptions such as weak admissibility, an alternative proof is presented in the following, using only the notion of Problem 3.3.4. 
Let us first state an auxilliary Lemma, proven in Appendix A.3.

Lemma 4.3.2. Let $x_{1}, x_{2}, r \in \mathbb{R}^{m}, y_{1}, y_{2}, s \in \mathbb{R}^{n}$ and $t \in[0,1]$. Define the convex combinations

$$
x_{t}:=t x_{1}+(1-t) x_{2}, \quad y_{t}:=t y_{1}+(1-t) y_{2}, \text { for } t \in[0,1]
$$

If we have

$$
\left\|x_{1}-x_{t}\right\|^{2}+C^{2}\left\|y_{1}-y_{t}\right\|^{2}-\left\|x_{1}-r\right\|^{2}-C^{2}\left\|y_{1}-s\right\|^{2} \geq 0
$$

and

$$
\left\|x_{2}-x_{t}\right\|^{2}+C^{2}\left\|y_{2}-y_{t}\right\|^{2}-\left\|x_{2}-r\right\|^{2}-C^{2}\left\|y_{2}-s\right\|^{2} \geq 0
$$

for some $C \in \mathbb{R}_{+}$. Then

$$
r=x_{t} \text { and } s=y_{t} \text {. }
$$

Let us first show that given the (NEPC) the set of fixed points of Algorithm 3.2.8 is convex.

Theorem 4.3.3. Let $F \in \mathbb{R}^{n \times m}, \kappa \in\{1,2\}, \beta \in \mathbb{R}_{+}$and let $(A, \underline{B}, \underline{\widetilde{B}}) \in \Gamma_{1+2 P}$ be input filters, with $(\underline{B}, \underline{\widetilde{B}})$ strongly admissible and satisfying the (NEPC). Let $\mathcal{X}$ be the set of fixed points of Algorithm 3.2.8. Then, $\mathcal{X}$ is convex.

Proof. If $\mathcal{X}=\emptyset$, it is convex. Consider, in the following $X \neq \emptyset$.

Let in the following $\left(U^{\dagger}, \underline{W}^{\dagger}, \underline{\lambda}^{\dagger}\right),\left(\widetilde{U}^{\dagger}, \underline{W}^{\dagger}, \underline{\tilde{\lambda}}^{\dagger}\right) \in \mathcal{X}$ be two fixed points of Algorithm 3.2.8 for the given input filters $(\underline{B}, \underline{\widetilde{B}})$. Denote their convex combination

$$
\left(U^{\dagger, t}, \underline{W}^{\dagger, t}, \underline{\lambda}^{\dagger, t}\right):=t\left(U^{\dagger}, \underline{W}^{\dagger}, \underline{\lambda}^{\dagger}\right)+(1-t)\left(\widetilde{U^{\dagger}}, \underline{\widetilde{W}}^{\dagger}, \underline{\tilde{\lambda}}^{\dagger}\right) \text {, for } t \in[0,1],
$$

we aim at showing $\left(U^{\dagger, t}, \underline{W}^{\dagger, t}, \underline{\lambda}^{\dagger, t}\right) \in \mathcal{X}$ for all $t \in[0,1]$, then $\mathcal{X}$ is convex.

First, we have by $(\underline{B}, \underline{\widetilde{B}})$ being strongly admissible and satisfying the (NEPC) and by $\mathcal{X} \neq \emptyset$ that Theorem 4.2.8 holds, i.e. Algorithm 3.2.8 with starting values $U_{t}^{(0)}=U^{\dagger, t}$ and $\underline{\lambda}_{t}^{(1)}=\underline{\lambda}^{\dagger, t}$ converges to a fixed point $\left(\ddot{U}^{t}, \underline{W}^{t}, \underline{\ddot{\lambda}}^{t}\right)$ for all $t \in[0,1]$. Observe that we have

$$
U^{(0)}=U^{(\dagger, t)}=\mathfrak{C}_{A}(F)+\underline{\mathfrak{C}}_{\underline{\widetilde{B}}}^{*}\left(\underline{W}^{\dagger, t}+\frac{1}{\beta} \underline{\lambda}^{(\dagger, t)}\right)=\mathfrak{C}_{A}(F)+\underline{\mathfrak{C}}_{\underline{\widetilde{B}}}^{*}\left(\underline{W}^{\dagger, t}+\frac{1}{\beta} \underline{\lambda}^{(1)}\right),
$$

by construction. Hence, by the use of (iii) and the repeated use of (ii) of Lemma 4.2.7 
(which is applicable by strong admissibility and the (NEPC)) we obtain

$$
\left\|\underline{\lambda}^{\dagger, t}-\underline{\lambda}^{\dagger}\right\|^{2}+\beta^{2}\left\|\underline{\mathfrak{C}}_{\underline{B}}\left(U^{\dagger, t}-U^{\dagger}\right)\right\|^{2}-\left\|\ddot{\lambda}^{t}-\underline{\lambda}^{\dagger}\right\|_{2}^{2}-\beta^{2}\left\|\underline{\mathfrak{C}}_{\underline{B}}\left(\ddot{U}^{t}-U^{\dagger}\right)\right\|_{2}^{2} \geq 0,
$$

and

$$
\left\|\underline{\lambda}^{\dagger, t}-\underline{\tilde{\lambda}}^{\dagger}\right\|^{2}+\beta^{2}\left\|\underline{\mathfrak{C}}_{\underline{B}}\left(U^{\dagger, t}-\widetilde{U}^{\dagger}\right)\right\|^{2}-\left\|\underline{\ddot{\lambda}}^{t}-\underline{\tilde{\lambda}}^{\dagger}\right\|_{2}^{2}-\beta^{2}\left\|\underline{\mathfrak{C}_{B}}\left(\ddot{U}^{t}-\widetilde{U}^{\dagger}\right)\right\|^{2} \geq 0,
$$

yielding via Lemma A.3.1:

$$
\ddot{\ddot{\lambda}}^{t}=\underline{\lambda}^{\dagger, t}, \quad \underline{\mathfrak{c}}_{\underline{B}}\left(\ddot{U}^{t}\right)=\underline{\mathfrak{C}}_{\underline{B}}\left(U^{\dagger, t}\right), \text { for all } t \in[0,1] .
$$

Since, $\left(\ddot{U}^{t}, \ddot{W}^{t}, \ddot{\lambda}^{t}\right),\left(U^{\dagger}, \underline{W}^{\dagger}, \underline{\lambda}^{\dagger}\right)$ and $\left(\widetilde{U^{\dagger}}, \underline{\tilde{W}}^{\dagger}, \tilde{\lambda}^{\dagger}\right)$ are fixed points of Algorithm 3.2.8 we have

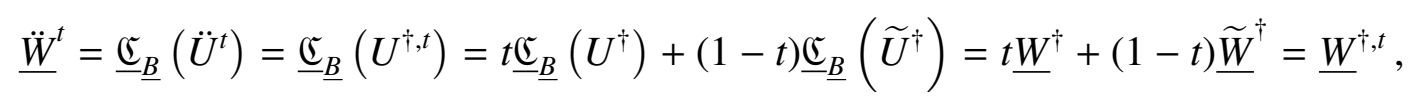

for all $t \in[0,1]$. In consequence of 4.3.1 we have $U^{\dagger, t}=\ddot{U}^{t}$, yielding $\left(U^{\dagger, t}, \underline{W}^{\dagger, t}, \underline{\lambda}^{\dagger, t}\right) \in$ $\mathcal{X}$ for all $t \in[0,1]$.

Showing uniqueness in the $U$-component will require Lemma 3.2.5, and thus strong admissibility and the (CPC) to hold for $(\underline{B}, \underline{\widetilde{B}})$.

Lemma 4.3.4. Let $F \in \mathbb{R}^{n \times m}, \kappa \in\{1,2\}, \beta \in \mathbb{R}_{+}$and $(A, \underline{B}, \underline{\widetilde{B}}) \in \Gamma_{1+2 P}$ be input filters, with $(\underline{B}, \underline{\widetilde{B}})$ strongly admissible and satisfying the $(N E P C)$. Let $\left(U^{\dagger}, \underline{W}^{\dagger}, \underline{\lambda}^{\dagger}\right)$ and $\left(\widetilde{U^{\dagger}}, \widetilde{W}^{\dagger}, \widetilde{\lambda}^{\dagger}\right)$ be two fixed points of Algorithm 3.2.8. Then

$$
\left\langle\underline{\lambda}^{\dagger}-\underline{\tilde{\lambda}}^{\dagger}, \underline{W}^{\dagger}-\underline{W}^{\dagger}\right\rangle=0
$$

Proof. Case 1: $\kappa=1$ :

For all fixed points $\left(U^{\dagger}, \underline{W}^{\dagger}, \underline{\lambda}^{\dagger}\right) \in \Gamma_{1+2 P}$ of Algorithm 3.2.8 we deduce via Lemma 3.3.5 that

$$
\underline{\mathfrak{C}}_{\underline{B}}\left(U^{\dagger}\right)=\underline{W}^{\dagger}=\mathrm{S}_{1}\left(\underline{\mathfrak{C}}_{\underline{B}}\left(U^{\dagger}\right)-\frac{1}{\beta} \underline{\lambda}^{\dagger} ; \frac{1}{\beta}\right),
$$


and hence,

$$
\lambda_{p}^{\dagger}[k, \ell] \begin{cases}=-1 & \text { if } \mathfrak{C}_{B_{p}}\left(U^{\dagger}\right)[k, \ell]>0 \\ \in[-1,1] & \text { if } \mathfrak{C}_{B_{p}}\left(U^{\dagger}\right)[k, \ell]=0, \quad \text { for all } p, k, \ell . \\ =1 & \text { if } \mathfrak{C}_{B_{p}}\left(U^{\dagger}\right)[k, \ell]<0\end{cases}
$$

Now define again

$$
\left(U^{\dagger, t}, \underline{W}^{\dagger, t}, \underline{\lambda}^{\dagger, t}\right):=t\left(U^{\dagger}, \underline{W}^{\dagger}, \underline{\lambda}^{\dagger}\right)+(1-t)\left(\widetilde{U}^{\dagger}, \underline{\widetilde{W}}^{\dagger}, \underline{\tilde{\lambda}}^{\dagger}\right) \text {, for } t \in[0,1],
$$

then, by strong admissibility and the (NEPC) we have using Theorem 4.3.3 that $\left(U^{\dagger, t}, \underline{W}^{\dagger, t}, \underline{\lambda}^{\dagger, t}\right)$ is a fixed point of Algorithm 3.2.8 for all $t \in[0,1]$.

Consider now an index $(k, \ell, p)$ such that

$$
\lambda_{p}^{\dagger}[k, \ell] \neq \tilde{\lambda}_{p}^{\dagger}[k, \ell]
$$

then by construction we have

$$
\lambda_{p}^{\dagger, t}[k, \ell] \in(-1,1) \text { for all } t \in(0,1)
$$

Via (4.3.2) we obtain

$$
{\underline{W^{\dagger}}}^{\dagger, t}[k, \ell]=\underline{\mathfrak{C}}_{\underline{B}}\left(U^{\dagger, t}\right)[k, \ell]=0 \text { for all } t \in(0,1),
$$

since all $\left(U^{\dagger, t}, \underline{W}^{\dagger, t}, \underline{\lambda}^{\dagger, t}\right)$ are fixed points of Algorithm 3.2.8. By construction of $\underline{W}^{\dagger, t}$ as a convex combination we conclude

$$
\underline{W}_{p}^{\dagger}[k, \ell]=0=\underline{\widetilde{W}}_{p}^{\dagger}[k, \ell] \text { for all }(k, \ell, p) \text { such that } \underline{\lambda}_{p}^{\dagger}[k, \ell] \neq \underline{\hat{\lambda}}_{p}^{\dagger}[k, \ell]
$$

Hence, by definition of the inner product we end up with the assertion.

Case 2: $\kappa=2$ :

For all fixed points $\left(U^{\dagger}, \underline{W}^{\dagger}, \underline{\lambda}^{\dagger}\right) \in \Gamma_{1+2 P}$ of Algorithm 3.2.8 we deduce via Lemma 3.3.5 that

$$
\underline{\mathfrak{C}}_{\underline{B}}\left(U^{\dagger}\right)=\underline{W}^{\dagger}=\mathrm{S}_{2}\left(\underline{\mathfrak{C}}_{\underline{B}}\left(U^{\dagger}\right)-\frac{1}{\beta} \underline{\lambda}^{\dagger} ; \frac{1}{\beta}\right),
$$


and hence,

$$
\left(\lambda_{p}^{\dagger}[k, \ell]\right)_{p=1}^{P}\left\{\begin{array}{ll}
=-\frac{\left(\mathfrak{C}_{B_{p}}(U)[k, \ell]\right]_{p=1}^{P}}{\left\|\left(\mathfrak{C}_{B_{p}}(U)[k, \ell]\right)_{p=1}^{P}\right\|} & \text { if }\left\|\left(\mathfrak{C}_{B_{p}}(U)[k, \ell]\right)_{p=1}^{P}\right\|>0 \\
\in \mathcal{B}_{1}(0) & \text { if }\left\|\left(\mathfrak{C}_{B_{p}}(U)[k, \ell]\right)_{p=1}^{P}\right\|=0
\end{array}, \quad \text { for all } k, \ell\right.
$$

Now define again

$$
\left(U^{\dagger, t}, \underline{W}^{\dagger, t}, \underline{\lambda}^{\dagger, t}\right):=t\left(U^{\dagger}, \underline{W}^{\dagger}, \underline{\lambda}^{\dagger}\right)+(1-t)\left(\widetilde{U}^{\dagger}, \underline{\widetilde{W}}^{\dagger}, \underline{\lambda}^{\dagger}\right), \text { for } t \in[0,1],
$$

then, by strong admissibility and the (NEPC) we have using Theorem 4.3.3 that $\left(U^{\dagger, t}, \underline{W}^{\dagger, t}, \underline{\lambda}^{\dagger}, t\right)$ is a fixed point of Algorithm 3.2.8 for all $t \in[0,1]$.

Consider now an index $(k, \ell)$ such that

$$
\left(\lambda_{p}^{\dagger}[k, \ell]\right)_{p=1}^{P} \neq\left(\widetilde{\lambda}_{p}^{\dagger}[k, \ell]\right)_{p=1}^{P}
$$

then by construction we have

$$
\left(\lambda_{p}^{\dagger}, t[k, \ell]\right)_{p=1}^{P} \in \mathcal{B}_{1}(0) \backslash \stackrel{\circ}{\mathcal{B}}_{1}(0) \text { for all } t \in(0,1),
$$

where $\stackrel{\circ}{\mathcal{B}}_{1}(0)$ is the boundary of $\mathcal{B}_{1}(0)$. Via (4.3.3) we obtain

$$
\left\|\left(W_{p}^{\dagger, t}[k, \ell]\right)_{p=1}^{P}\right\|=\left\|\left(\mathfrak{C}_{B_{p}}\left(U^{\dagger, t}\right)[k, \ell]\right)_{p=1}^{P}\right\|=0 \text { for all } t \in(0,1)
$$

since all $\left(U^{\dagger, t}, \underline{W}^{\dagger, t}, \underline{\lambda}^{\dagger, t}\right)$ are fixed points of Algorithm 3.2.8. By construction of $\underline{W}^{\dagger, t}$ as a convex combination we conclude

$$
\left\|\left(\underline{W}_{p}^{\dagger}[k, \ell]\right)_{p=1}^{P}\right\|=0=\left\|\left(\underline{\widetilde{W}}_{p}^{\dagger}[k, \ell]\right)_{p=1}^{P}\right\|
$$

for all $(k, \ell)$ such that

$$
\left(\underline{\lambda}_{p}^{\dagger}[k, \ell]\right)_{p=1}^{P} \neq\left(\tilde{\lambda}_{p}^{\dagger}[k, \ell]\right)_{p=1}^{P} .
$$

Hence, by definition of the inner product we end up with the assertion.

Theorem 4.3.5. Let $F \in \mathbb{R}^{n \times m}, \kappa \in\{1,2\}, \beta \in \mathbb{R}_{+}$and let $(A, \underline{B}, \underline{\widetilde{B}}) \in \Gamma_{1+2 P}$ be input filters, with $(\underline{B}, \underline{\widetilde{B}})$ strongly admissible and satisfying the $(C P C)$. Then Algorithm 3.2.8 converges to a fixed point with unique $(U, \underline{W})$.

Proof. By $(\underline{B}, \underline{\widetilde{B}}) \in \Gamma_{1+2 P}$ being strongly admissible input filters that satisfy the (CPC) we have by Corollary 4.2.9 that Algorithm 3.2.8 converges to a fixed point. Left to show 
is that for any two fixed points of Algorithm 3.2.8 their $(U, \underline{W})$-components coincide.

Let in the following $\left(U^{\dagger}, \underline{W}^{\dagger}, \underline{\lambda}^{\dagger}\right) \in \Gamma_{1+2 P}$ and $\left(\widetilde{U}^{\dagger}, \underline{\widetilde{W}}^{\dagger}, \underline{\tilde{\lambda}}^{\dagger}\right) \in \Gamma_{1+2 P}$ be two fixed points of Algorithm 3.2.8.

Define

$$
(\breve{U}, \underline{\breve{W}}, \underline{\breve{\lambda}}):=\left(U^{\dagger}, \underline{W}^{\dagger}, \underline{\lambda}^{\dagger}\right)-\left(\widetilde{U}^{\dagger}, \underline{\widetilde{W}}^{\dagger}, \underline{\tilde{\lambda}}^{\dagger}\right),
$$

then by Lemma 4.3.4 we have

$$
\langle\underline{\breve{\lambda}}, \underline{\breve{W}}\rangle=0 \text {. }
$$

Furthermore we have by the fixed point relations,

$$
\underline{\breve{W}}=\underline{W}^{\dagger}-\underline{\widetilde{W}}^{\dagger}=\underline{\mathfrak{c}}_{\underline{B}}\left(U^{\dagger}-\widetilde{U}^{\dagger}\right)=\underline{\mathfrak{c}}_{\underline{B}}(\breve{U})
$$

yielding,

$$
\begin{aligned}
\underline{\breve{W}} & =\underline{\mathfrak{C}}_{\underline{B}}(\breve{U})=\underline{\mathfrak{C}}_{\underline{B}}\left(U^{\dagger}\right)-\underline{\mathfrak{C}}_{\underline{B}}\left(\widetilde{U}^{\dagger}\right) \\
& =\underline{\mathfrak{C}}_{\underline{B}}\left(\mathfrak{C}_{A}(F)-\mathfrak{C}_{A}(F)+\underline{\mathfrak{C}}_{\widetilde{\underline{B}}}^{*}\left(\underline{W}^{\dagger}-\widetilde{W}^{\dagger}+\frac{1}{\beta}\left(\underline{\lambda}^{\dagger}-\underline{\tilde{\lambda}}^{\dagger}\right)\right)\right) \\
& =\underline{\mathfrak{C}}_{\underline{B}} \underline{\mathfrak{C}}_{\underline{\widetilde{B}}}^{*}\left(\underline{\breve{W}}+\frac{1}{\beta} \underline{\breve{\lambda}}\right) .
\end{aligned}
$$

Consider now,

$$
\begin{aligned}
& 0 \stackrel{(C P C)}{\geq}-\left\langle\frac{1}{\beta} \underline{\mathfrak{C}}_{\underline{B}} \underline{\mathfrak{C}}_{\underline{\underline{B}}}^{*}(\underline{\breve{\lambda}}), \underline{\breve{\lambda}}\right\rangle \stackrel{(4.3 .6)}{=}\left\langle\underline{\mathfrak{C}_{\underline{B}}} \underline{\widetilde{C}}_{\underline{\underline{B}}}^{*}(\underline{\breve{W}})-\underline{\breve{W}}, \underline{\breve{\lambda}}\right\rangle \\
& \stackrel{(4.3 .4)}{=}\left\langle\underline{\mathfrak{C}}_{\underline{B}} \underline{\mathfrak{C}}_{\underline{\widetilde{B}}}^{*}(\underline{\breve{W}}), \underline{\breve{\lambda}}\right\rangle \\
& =\left\langle\underline{W}, \underline{\mathfrak{C}_{\widetilde{B}}} \underline{\underline{C}}_{\underline{B}}^{*}(\underline{\breve{\lambda}})\right\rangle \\
& \stackrel{3.2 .3}{=}\left\langle\underline{\underline{W}}, \underline{\mathfrak{C}}_{\underline{B}} \underline{\underline{\mathfrak{C}_{\underline{B}}^{*}}}(\underline{\underline{\lambda}})\right\rangle \\
& \stackrel{(4.3 .6)}{=}\left\langle\underline{\breve{W}}, \beta\left(\underline{\breve{W}}-\underline{\mathfrak{C}}_{\underline{B}} \underline{\mathfrak{C}_{\overparen{B}}^{*}}(\underline{\breve{W}})\right)\right\rangle \\
& =\beta\left(\|\underline{\underline{W}}\|^{2}-\left\|\left(\underline{\mathfrak{C}_{\underline{B}}} \underline{\underline{E}}_{\underline{\underline{B}}}^{*}\right)^{1 / 2} \underline{\underline{W}}\right\|^{2}\right) \begin{array}{c}
(C P C) \\
\geq 0
\end{array} \text {. }
\end{aligned}
$$

We conclude at once,

$$
\underline{\mathfrak{C}}_{\underline{B}} \underline{\mathfrak{C}}_{\underline{\widetilde{B}}}^{*}(\underline{\breve{\lambda}})=0 .
$$

Let $U:=\underline{\mathfrak{C}}_{\widetilde{B}}^{*}(\underline{\lambda})$ and $U \in \operatorname{ker}\left(\underline{\mathfrak{C}}_{\underline{B}}\right)$. Then we have

$$
\underline{\mathfrak{C}}_{\underline{B}}(U)=\underline{0} \Rightarrow \widehat{B}_{p}[k, \ell] \widehat{U}[k, \ell]=0 \text { for all } 0 \leq k \leq n-1 \text { and } 0 \leq \ell \leq m-1 \text {, }
$$


yielding,

$$
\widehat{U}[k, \ell]=0 \text { for all } k, \ell \text { such that } \exists p \in\{1,2, \ldots, P\} \text { with } \widehat{B}_{p}[k, \ell] \neq 0 \text {. }
$$

Let now $(k, \ell)$ be such that $\widehat{U}[k, \ell] \neq 0$, then we have for all $p \in\{1,2, \ldots, P\}$ that $\widehat{B}_{p}[k, \ell]=0$, hence by weak admissibility (given by strong admissibility) we have $\widehat{\widetilde{B}}_{p}[k, \ell]=0$. This yields,

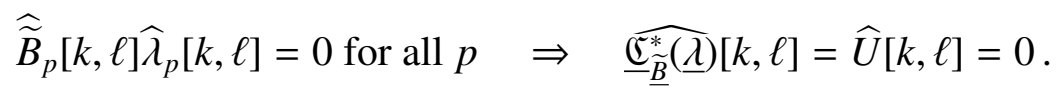

Hence, $U$ is the zero matrix. Thus we can infer from (4.3.7) that

$$
\underline{\mathfrak{C}}_{\widetilde{B}}^{*}(\underline{\lambda})=0 .
$$

Finally, we deduce

$$
\begin{aligned}
\breve{U}=U^{\dagger}-\widetilde{U}^{\dagger} & =\mathbb{C}_{A}(F)-\mathfrak{C}_{A}(F)+\underline{\mathfrak{C}}_{\underline{\underline{B}}}^{*}\left(\underline{W}^{\dagger}-\underline{\widetilde{W}}^{\dagger}+\frac{1}{\beta}\left(\underline{\lambda}^{\dagger}-\underline{\tilde{\lambda}}^{\dagger}\right)\right) \\
& =\underline{\mathfrak{C}}_{\underline{\widetilde{B}}}^{*}\left(\underline{\breve{W}}+\frac{1}{\beta} \underline{\breve{\lambda}}\right)=\underline{\mathfrak{C}}_{\underline{\underline{B}}}^{*}(\underline{\breve{W}}) \\
\stackrel{(4.3 .5)}{\Rightarrow} \breve{U} & =\underline{\mathbb{C}}_{\underline{\underline{C}}}^{*} \underline{\mathfrak{C}}_{\underline{B}}(\breve{U}),
\end{aligned}
$$

By Lemma 3.2.5 and the assumption that $(\underline{B}, \underline{\widetilde{B}})$ satisfy the (CPC) we conclude

$$
\breve{U}=0 \text { and in consequence } \underline{\breve{W}}=\underline{0} \text {. }
$$




\section{CHAPTER 5}

\section{Experimental Results}

In this chapter benefits of Algorithm 3.2.8 are analysed. Denoising experiments as well as novel decompositions into cartoon and texture components are considered. In particular we try to understand if there lies gain in generalizing Problem 3.1.8 to Problem 3.2.9. Since an exhaustive analysis ranging over all possible decompositions of Problem 3.1.8 and Problem 3.2.9, respectively, would exceed the scope of this thesis, we consider specific families of input filters related to the popular $T V-\ell^{2}$-problem.

It is often easier to define a matrix convolution $\mathfrak{C}_{A}$ via the Fourier transform of its convolution matrix $\widehat{A}$. To do this efficiently let us introduce some additional notation that will ease calculations in this chapter, and Appendix $\mathrm{C}$

Convention 5.0.6. For an entry $[k, \ell]$ with $0 \leq k \leq n-1$ and $0 \leq \ell \leq m-1$ set $\omega_{k}, \omega_{\ell} \in[-\pi, \pi)$ to be

$$
\omega_{k}:=\left\{\begin{array}{ll}
\frac{2 \pi k}{n} & \text { if } k<\frac{n}{2} \\
-2 \pi+\frac{2 \pi k}{n} & \text { else }
\end{array} \quad \text { and } \quad \omega_{\ell}:=\left\{\begin{array}{ll}
\frac{2 \pi \ell}{m} & \text { if } \ell<\frac{m}{2} \\
-2 \pi+\frac{2 \pi \ell}{m} & \text { else }
\end{array} .\right.\right.
$$

\subsection{Denoising Experiments}

Consider the imaging model

$$
F=U+\varepsilon, \quad \text { where } \varepsilon \sim \mathcal{N}\left(0, \sigma^{2} \Im\right),
$$

where $\mathfrak{S}$ is some covariance matrix with matrix-valued entries and $\sigma \in \mathbb{R}_{+}$. We compare three models for denoising based on Problem 3.1.8 and 3.2.9 in three experiments, cf. Table 5.1 and 5.2.

The first model considered is the one-parameter classical $T V-\ell^{2}$-model, as a representative of Problem 3.1.8. The second and third model considered are augmentations of the $T V-\ell^{2}$ model, each adding two-parameters. Both augmented models are subfamilies of 
Problem 3.2.9, one featuring strong admissibility $(\underline{B}, \underline{\widetilde{B}})$, while the other one only weak admissibility. The aim is to investigate whether improved denoising results, measured by the peak signal-to-noise ratio (PSNR), can be obtained by the generalized models. The following three denoising experiments are considered:

(1) In the first experiment, $U$ and $\varepsilon$ of (5.1.1) are known. We optimize the parameter choice for all models with respect to maximizing the PSNR. Can the generalized models improve in terms of PSNR on the classical model?

(2) The second experiment, considers the dependence of the improvement in PSNR in (1) on knowing $\varepsilon$. Let now $U$ and $\sigma^{2} \subseteq$ of (5.1.1) be known. We use the optimized parameters of (1) for all models and the respective covariance operators $\sigma^{2} \subseteq$. Do the generalized models still score higher PSNR's on the classical model on $\left\{U+\varepsilon_{t}\right\}_{t=1}^{T}$, featuring newly drawn $\varepsilon_{t} \sim \mathcal{N}\left(0, \sigma^{2} \Xi\right)$ ?

(3) The third experiment, considers the dependence of the improvement in PSNR in (1) and (2) on the image $U$. Let now $\left\{U_{t}\right\}_{t=1}^{T_{1}+T_{2}}$ be a database of similar images, here fingerprint images, and let $\sigma^{2} \subseteq$ be known. Given training images $U_{1}, \ldots, U_{T_{1}}$, train parameters for all models on the training images with added noise $\left\{\varepsilon_{t}\right\}_{t=1}^{T_{1}}$. Can an improvement in PSNR for the generalized models be observed when applied to the test set $\left\{U_{t+T_{1}}+\varepsilon_{t+T_{1}}\right\}_{t=1}^{T_{2}}$, featuring new realizations $\varepsilon_{t} \sim \mathcal{N}\left(0, \sigma^{2} \mathfrak{S}\right)$ ?

Table 5.1

Overview over the known and unknown matrices of the Experiments

\begin{tabular}{c|c|c} 
Experiment & Known & Unknown \\
\hline 1 & $U, \varepsilon$ & - \\
2 & $U, \varepsilon_{0}$ & $\left\{\varepsilon_{t}\right\}_{t=1}^{T}$ \\
3 & $\left\{U_{t}, \varepsilon_{t}\right\}_{t=1}^{T_{1}}$ & $\left\{U_{t+T_{1}}, \varepsilon_{t+T_{1}}\right\}_{t=1}^{T_{2}}$
\end{tabular}

\subsubsection{Compared Models and the PSNR}

In the following we detail the compared models taking part in the experiments, denoted by Set-Up 5.1.2, Set-Up 5.1.3 and Set-Up 5.1.4, respectively. Throughout this chapter we set $\kappa=2$, as in the classical $T V-\ell^{2}$-problem, and $\beta=0.1$. For an overview of the considered input filters see Table 5.2.

Remark 5.1.1. Let $\mu \in \mathbb{R}_{+}$and $\beta \in \mathbb{R}_{+}$be fixed, and let $\underline{D}$ be the matrix-family giving rise to the discrete gradient $\underline{\mathfrak{E}}_{D}$, cf. Example 2.1.12. Then, there exist by Lemma 3.2.12 


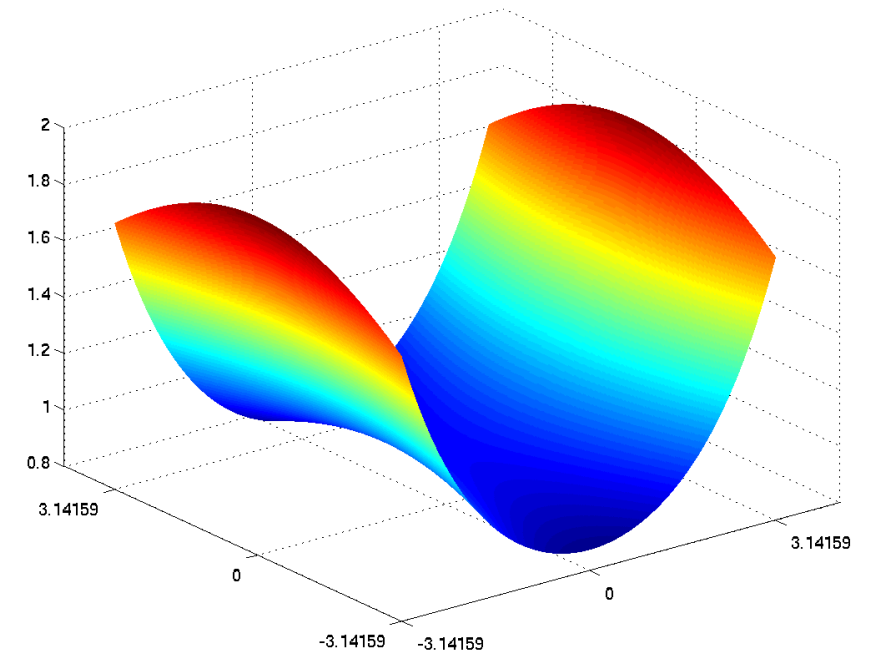

Figure 5.1: Plot of $\widehat{V}$ of Set-Up 5.1.3 with $y_{1}=0.07$ and $y_{2}=-0.014$.

input filters $\left(A_{T V}, \underline{B}_{T V}, \underline{\widetilde{B}}_{T V}\right)_{\mu}$, only depending on $\mu$ and $\underline{D}$ (since $\beta$ is fixed in this chapter), such that Problem 3.2.9 is equivalent to the $T V-\ell^{2}$-problem. In the following we call $\left(A_{T V}, \underline{B}_{T V}, \underline{\widetilde{B}}_{T V}\right)_{\mu}$ the $T V-\ell^{2}$-input filters.

Set-Up 5.1.2 (Classical $T V-\ell^{2}$-problem). We consider the set of all $T V-\ell^{2}$ input filters,

$$
\left\{\left(A_{\mathrm{TV}}, \underline{B}_{\mathrm{TV}}, \underline{\widetilde{B}}_{\mathrm{TV}}\right)_{\mu}: \mu \in \mathbb{R}_{+}\right\} .
$$

Recall that $\kappa$ and $\beta$ are fixed and set to 2 and 0.1 , respectively.

Set-Up 5.1.3. (strongly admissible) Let for $\mu \in \mathbb{R}_{+}$the $T V-\ell^{2}$-input filters be given by $\left(A_{\mathrm{TV}}, \underline{B}_{\mathrm{TV}}, \underline{\widetilde{T}}_{\mathrm{TV}}\right)_{\mu}(\kappa=2$ and $\beta=0.1)$. Furthermore, define for $y_{1}, y_{2} \in \mathbb{R}$ the matrices $V, \widetilde{V}$ by their Fourier transforms

$$
\widehat{V}[k, \ell]:=\exp \left(-y_{1} \omega_{k}^{2}-y_{2} \omega_{\ell}^{2}\right) \quad 0 \leq k \leq n-1 \text { and } 0 \leq \ell \leq m-1,
$$

and

$$
\widehat{\widetilde{V}}[k, \ell]:=\exp \left(y_{1} \omega_{k}^{2}+y_{2} \omega_{\ell}^{2}\right) \quad 0 \leq k \leq n-1 \text { and } 0 \leq \ell \leq m-1,
$$

using Convention 5.0.6 and set $\underline{V}=(V, V), \underline{\widetilde{V}}=(\widetilde{V}, \widetilde{V}) \in \Gamma_{2}$, Figure 5.1 depicts $\widehat{V}$ on $[-\pi, \pi)^{2}$ for $y_{1}=0.07$ and $y_{2}=-0.014$. Since concatinations of matrix-convolutions are again matrix-convolutions let $\underline{B}_{\mathrm{VTV}}, \underline{\widetilde{B}}_{\mathrm{VTV}} \in \Gamma_{2}$ be the matrix-families giving rise to the matrix-family convolutions $\underline{\underline{C}}_{\underline{V}} \underline{\underline{C}}_{\underline{B}_{\mathrm{TV}}}$ and $\underline{\underline{\mathfrak{C}}} \underline{\underline{\underline{C}}} \underline{\underline{\mathbb{C}}}_{\widetilde{\mathrm{T}}_{\mathrm{TV}}}$, respectively. Consider,

$$
\left\{\left(A_{\mathrm{TV}}, \underline{B}_{\mathrm{VTV}}, \underline{\widetilde{B}}_{\mathrm{VTV}}\right)_{\mu, y_{1}, y_{2}}: \mu \in \mathbb{R}_{+} \text {and } y_{1}, y_{2} \in \mathbb{R}\right\} .
$$


Table 5.2

Resulting Operators in Algorithm 3.2.8

\begin{tabular}{|c|c|c|c|}
\hline Operator & $\begin{array}{c}\text { Set-Up 5.1.2 } \\
\text { (classical) }\end{array}$ & $\begin{array}{c}\text { Set-Up } 5.1 .3 \\
\text { (strongly) }\end{array}$ & $\begin{array}{l}\text { Set-Up 5.1.4 } \\
\text { (weakly) }\end{array}$ \\
\hline $\mathfrak{C}_{A}$ & $\mu\left(\mu+\beta \underline{\mathfrak{C}}_{\underline{D}}^{*} \underline{\mathfrak{C}}_{\underline{D}}\right)^{-1}$ & $\mu\left(\mu+\beta \underline{\mathfrak{C}}_{\underline{D}}^{*} \underline{\mathfrak{C}}_{\underline{D}}\right)^{-1}$ & $\mu\left(\mu+\beta \underline{\mathbb{C}}_{\underline{D}}^{*} \underline{\mathfrak{C}}_{\underline{D}}\right)^{-1}$ \\
\hline$\underline{\mathfrak{C}}_{\underline{B}}$ & $\underline{\mathfrak{C}}_{\underline{D}}$ & $\underline{\underline{C}}_{\underline{\underline{C}}} \underline{\mathfrak{C}}_{\underline{D}}$ & $\underline{\underline{C}}_{R} \underline{\mathfrak{C}}_{\underline{D}}$ \\
\hline$\underline{\mathfrak{C}}_{\underline{B}}^{*}$ & $\beta\left(\mu+\beta \underline{\mathfrak{C}}_{\underline{D}}^{*} \underline{\mathfrak{C}}_{\underline{D}}\right)^{-1} \underline{\mathfrak{C}}_{\underline{D}}^{*}$ & $\beta\left(\mu+\beta \underline{\mathfrak{C}}_{\underline{D}}^{*} \underline{\mathfrak{C}}_{\underline{D}}\right)^{-1} \mathfrak{C}_{\widetilde{V}} \underline{\mathfrak{C}}_{\underline{D}}^{*}$ & $\beta\left(\mu+\beta \underline{\mathbb{C}}_{\underline{D}}^{*} \underline{\mathfrak{C}}_{\underline{D}}\right)^{-1} \underline{\mathfrak{C}}_{\underline{D}}^{*} \underline{\mathfrak{C}}_{\widetilde{R}}$ \\
\hline
\end{tabular}

Set-Up 5.1.4. (weakly admissible) Let for $\mu \in \mathbb{R}_{+}$the $T V-\ell^{2}$-input filters be given by $\left(A_{\mathrm{TV}}, \underline{B}_{\mathrm{TV}}, \underline{\widetilde{B}}_{\mathrm{TV}}\right)_{\mu}(\kappa=2$ and $\beta=0.1)$. Furthermore, define for $r_{1}, r_{2} \in \mathbb{R}_{+}$the matrix-families $\underline{R}, \underline{\widetilde{R}} \in \Gamma_{2}$ for $p=1,2$ by the Fourier transforms of their matrix-entries

$$
\widehat{R}_{1}[k, \ell]:=r_{1}^{-1}, \quad \widehat{R}_{2}[k, \ell]:=r_{2}^{-1}, \quad \text { for all } 0 \leq k \leq n-1 \text { and } 0 \leq \ell \leq m-1,
$$

and,

$$
\widehat{\widetilde{R}}_{1}[k, \ell]:=r_{1} \quad \widehat{\widetilde{R}}_{2}[k, \ell]:=r_{2}, \quad \text { for all } 0 \leq k \leq n-1 \text { and } 0 \leq \ell \leq m-1
$$

Let now $\underline{B}_{\mathrm{RTV}}, \underline{\widetilde{B}}_{\mathrm{RTV}} \in \Gamma_{2}$ be the matrix-families giving rise to the matrix-family convolutions $\underline{\underline{C}}_{\underline{R}} \underline{\mathfrak{C}}_{\underline{\mathrm{T}}_{\mathrm{TV}}}$ and $\underline{\underline{\mathfrak{C}}}_{\underline{\underline{R}}} \underline{\mathfrak{C}}_{\underline{\underline{B}}_{\mathrm{TV}}}$, respectively. Consider,

$$
\left\{\left(A_{\mathrm{TV}}, \underline{B}_{\mathrm{RTV}}, \underline{\widetilde{B}}_{\mathrm{RTV}}\right)_{\mu, r_{1}, r_{2}}: \mu, r_{1}, r_{2} \in \mathbb{R}_{+}\right\} .
$$

Our experiments will be evaluated using the peak signal-to-noise ratio (PSNR), an often considered performance measures in image-denoising [Bov09]. It is closely related to the mean-squared error (MSE), see its Definition below.

Definition 5.1.5 ([Bov09][p.146]). Let $U \in \mathbb{R}^{n \times m}$ be an input image and $U_{\text {approx }} \in \mathbb{R}^{n \times m}$ be an approximation to $U$. We define the peak signal-to-noise ratio (PSNR) by

$$
\operatorname{PSNR}\left(U_{\text {approx }}, U\right)=10 \log _{10}\left(\frac{\operatorname{MAX}^{2}}{\operatorname{MSE}\left(U_{\text {approx }}, U\right)}\right),
$$

where MAX is the maximum grey-value (for 8-bit images used here it is 255) and the MSE is the mean-squared error given by

$$
\operatorname{MSE}\left(U_{\text {approx }}, U\right):=\frac{1}{m n} \sum_{k=0}^{n-1} \sum_{\ell=0}^{m-1}\left(U_{\text {approx }}[k, \ell]-U[k, \ell]\right)^{2}=\frac{1}{m n}\left\|U_{\text {approx }}-U\right\|^{2} .
$$




\subsubsection{Images and Covariance Operators}

In Experiments 1 and 2, we consider the publicly available (e.g.[SIP, hle]) test images (a) to (g) of Figure 5.2 as $U$ in (5.1.1). In Experiment 3 we use a subset of the publicly available fingerprint database FVC2000 DB1 (Maio et al. [MMWJ02]). This database contains 880 fingerprints in total, 8 repeated prints of 110 individuals, an example print is shown in (h) of Figure 5.2. We use the first fingerprint of each individual in Experiment 3, to include only different fingerprint images obtaining a total of 110 images. Training is done on the first 10, while the test set is comprised of the remaining 100 images.

For Experiment 1, five different covariance operators are consider, listed in the following.

(i) No correlation: Covariance is the identity operator, i.e. $\mathfrak{S}=\mathfrak{E}$.

(ii) Vertical correlation: A random matrix with iid normal entries is blurred with a convolution matrix $Z_{y} \in \mathbb{R}^{n \times m}$ defined by

$$
Z_{y}=\frac{1}{20}\left(\begin{array}{cccccc}
4 & 1 & 0 & \cdots & 0 & 1 \\
4 & 0 & & & & 0 \\
2 & 0 & \ddots & & & \vdots \\
1 & 0 & & & & \\
0 & & & & & \\
\vdots & & & & & \\
0 & & & & & \\
1 & 0 & & & & \vdots \\
2 & 0 & & & \ddots & 0 \\
4 & 0 & \cdots & \cdots & 0 & 0
\end{array}\right) \in \mathbb{R}^{n \times m}
$$

yielding, the covariance operator $\mathfrak{S}=\mathfrak{C}_{Z_{y}} \mathfrak{C}_{Z_{y}}^{*}$.

(iii) Horizontal correlation: A random matrix with iid normal entries is blurred with 


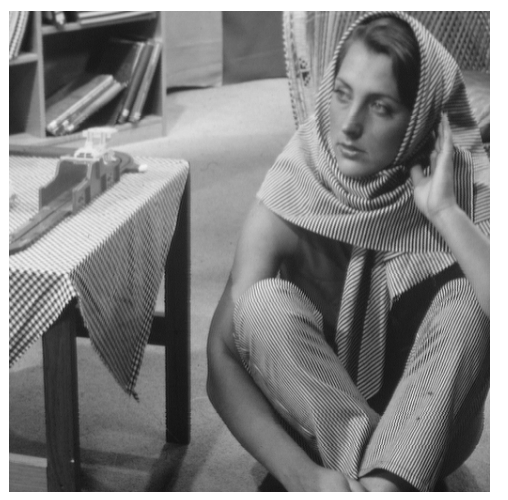

(a)

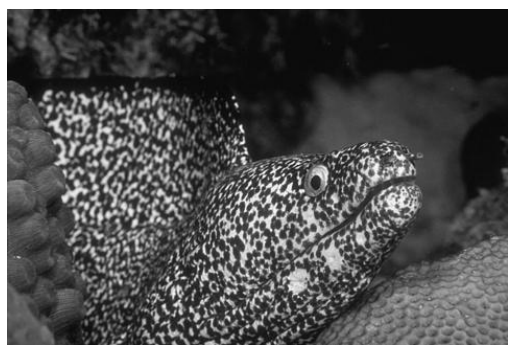

(c)

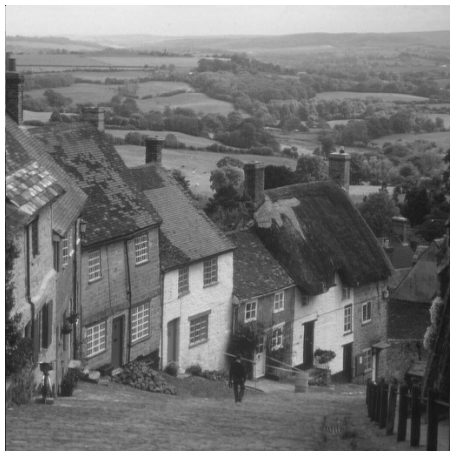

(e)

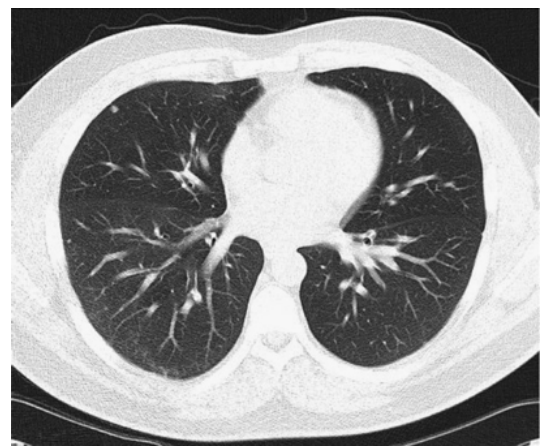

$(\mathrm{g})$

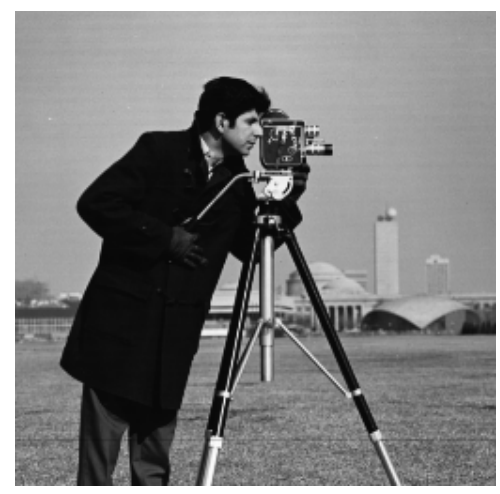

(b)

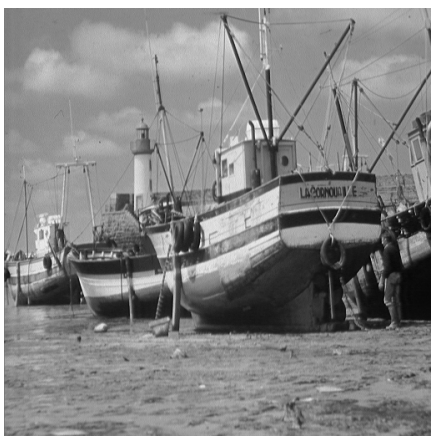

(d)

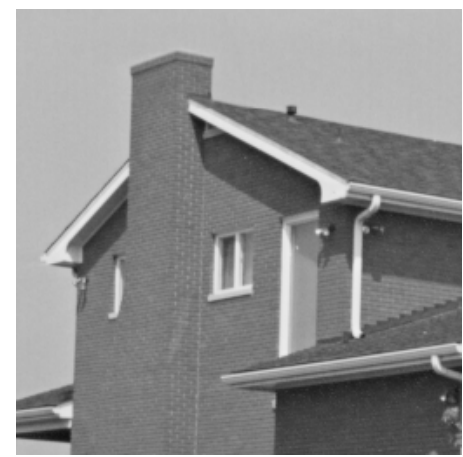

(f)

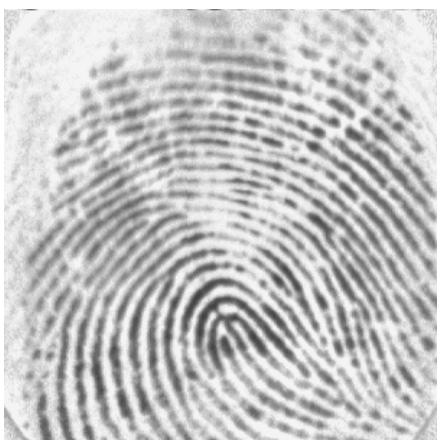

(h)

Figure 5.2: Publicly available test images (e.g. [SIP, hle]) used in the experiments: barbara (a), cameraman (b), fish (c), boat (d), goldhill (e), house (f), lung (g) and a fingerprint example of FVC2000 DB1 (h) 
a convolution matrix $Z_{x} \in \mathbb{R}^{n \times m}$ defined by

$$
Z_{x}=\frac{1}{20}\left(\begin{array}{cccccccccc}
4 & 4 & 2 & 1 & 0 & \cdots & 0 & 1 & 2 & 4 \\
1 & 0 & 0 & 0 & 0 & & & 0 & 0 & 0 \\
0 & 0 & \ddots & & & & & 0 & 0 \\
\vdots & & & & & & & & \vdots \\
0 & 0 & & & & & & \ddots & 0 \\
1 & 0 & \cdots & & & & \cdots & 0 & 0
\end{array}\right) \in \mathbb{R}^{n \times m}
$$

yielding, the covariance operator $\mathfrak{S}=\mathfrak{C}_{Z_{x}} \mathfrak{C}_{Z_{x}}^{*}$.

(iv) horizontal and vertical correlation: A random matrix with iid normal entries is blurred with the convolution matrix $Z_{x}$ and additionally with the convolution matrix $Z_{y}$, yielding the covariance operator $\mathfrak{S}=\mathfrak{C}_{Z_{y}} \mathfrak{C}_{Z_{x}} \mathfrak{C}_{Z_{x}} \mathfrak{C}_{Z_{y}}^{*}$.

(v) Diagonal correlation: A random matrix with iid normal entries is blurred with a convolution matrix $Z_{d} \in \mathbb{R}^{n \times m}$ defined by

$$
Z_{d}=\frac{1}{22}\left(\begin{array}{cccccccccc}
4 & 1 & 0 & 0 & 0 & \cdots & & \cdots & 0 & 1 \\
1 & 4 & 0 & 0 & 0 & & & & & 0 \\
0 & 0 & 2 & 0 & 0 & & & & & \vdots \\
0 & 0 & 0 & 1 & 0 & & & & & \\
0 & 0 & 0 & 0 & 0 & & & & & \\
\vdots & & & & & \ddots & & & & \vdots \\
& & & & & & 0 & 0 & 0 & 0 \\
\vdots & & & & & & 0 & 1 & 0 & 0 \\
0 & & & & & & 0 & 0 & 2 & 0 \\
1 & 0 & \cdots & & & \cdots & 0 & 0 & 0 & 4
\end{array}\right) \in \mathbb{R}^{n \times m}
$$

yielding, the covariance operator $\mathfrak{S}=\mathfrak{C}_{Z_{d}} \mathfrak{C}_{Z_{d}}^{*}$.

While for Experiment 1 we use all of the above described covariances, with varying $\sigma \in \mathbb{R}_{+}$, in Experiment 2 and 3 we will narrow the choice down to the most interesting ones, to allow for a compact presentation.

\subsubsection{Parameter Optimization and Protocols}

Choosing an optimal $\mu$ in Set-Up 5.1.2 to obtain a maximal PSNR is not straight-forward. Let $U_{\mathrm{TV}}^{\mu, F}$ be the solution of Algorithm 3.2.8 for the $T V-\ell^{2}$ input filters with $\mu \in \mathbb{R}_{+}$ 
and for a corrupted input $F \in \mathbb{R}^{n \times m}$. Then, the function

$$
f_{\mathrm{PSNR}}(\mu, F, U)=\operatorname{PSNR}\left(U, U_{\mathrm{TV}}^{\mu, F}\right)
$$

is usually observed to have a unique global maximum and no local maxima, see Aubert and Kornprobst [AK06][p.88]. Note, that in the experiments conducted we found this to be true. Therefore we use a bisection algorithm, see for example Boyd and Vandenberghe [BV04], detailed in Algorithm 5.1.6 to obtain an optimal $\mu$ for a given clean image $U$ and its noisy counterpart $F=U+\varepsilon$.

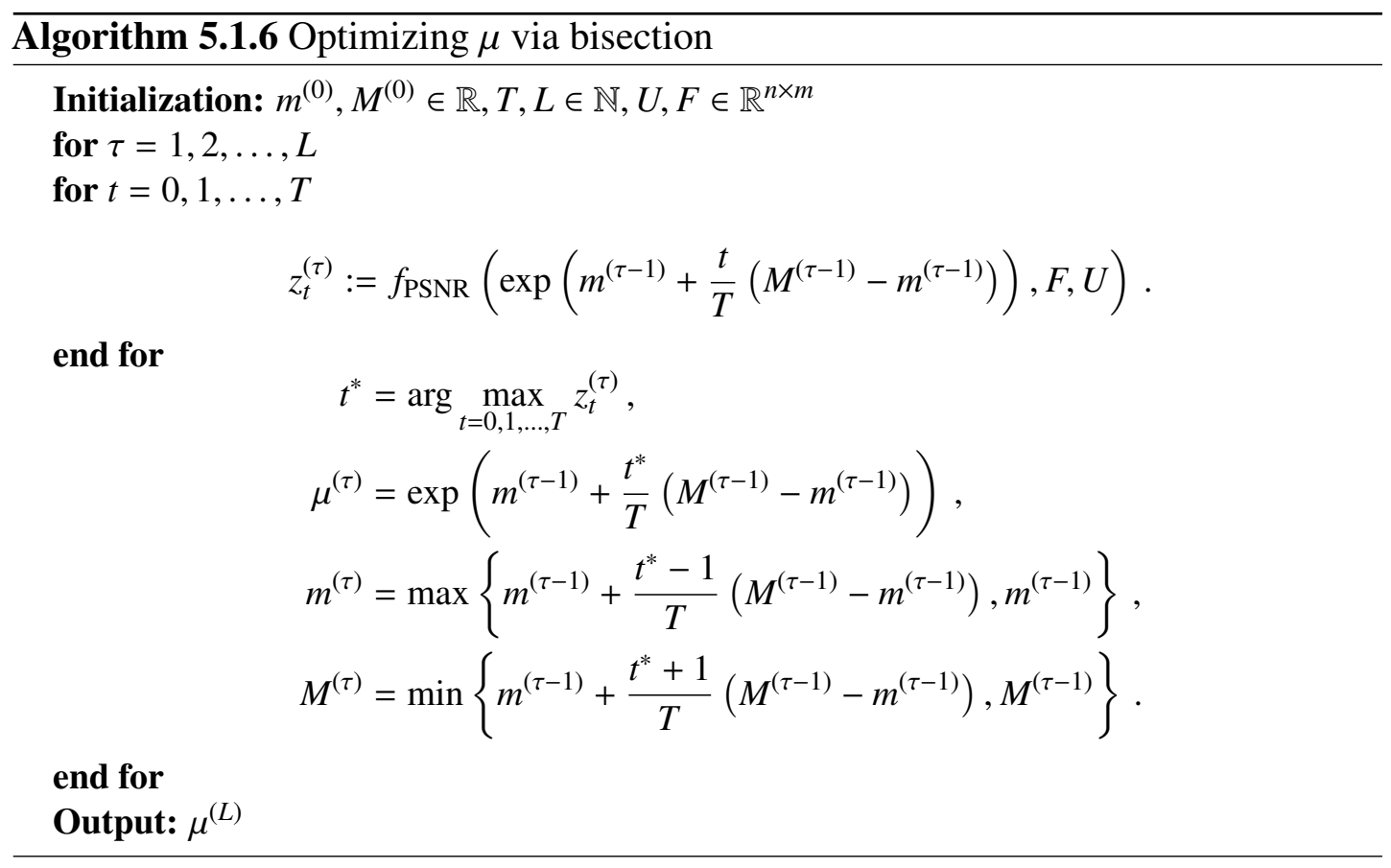

Within the limited scope of this thesis we use the same $\mu$ as optimized for Set-Up 5.1.2 also in Set-Up 5.1.3 and 5.1.4. The remaining parameters $r_{1}, r_{2}$ and $y_{1}, y_{2}$ are then optimized via a grid search over educated guesses covering the first and third quadrant of a rectangle centred at $(0,0)$. The following 20 values for Set-Up 5.1.3

$$
\begin{array}{r}
\left(y_{1}, y_{2}\right) \in\{(-0.07,0.014),(-0.042,0.014),(-0.014,0.014),(-0.07,0.042), \\
(-0.042,0.042),(-0.014,0.042),(-0.07,0.07),(-0.042,0.07), \\
(-0.014,0.07),(0.07,-0.014),(0.042,-0.014),(0.014,-0.014), \\
(0.07,-0.042),(0.042,-0.042),(0.014,-0.042),(0.07,-0.07), \\
(0.042,-0.07),(0.014,-0.07),(0.014,0.014),(-0.014,-0.014)\},
\end{array}
$$


Table 5.3

Fixed Parameters for all Experiments.

\begin{tabular}{c|c|c} 
Algorithm & Parameter & Value \\
\hline \multirow{2}{*}{ Algorithm 3.2.8 } & $\beta$ & 0.1 \\
& $\kappa$ & 2 \\
\hline \multirow{3}{*}{ Algorithm 5.1.6 } & $m^{(0)}$ & -10 \\
& $M^{(0)}$ & 0 \\
& $T$ & 10 \\
$L$ & 5
\end{tabular}

and the following 20 values for Set-Up 5.1.4

$$
\begin{array}{r}
\left(\log \left(r_{1}\right), \log \left(r_{2}\right)\right) \in\{(-0.3,0.06),(-0.18,0.06),(-0.06,0.006),(-0.3,0.18), \\
(-0.18,0.18),(-0.06,0.18),(-0.3,0.3),(-0.18,0.3), \\
(-0.06,0.3),(0.3,-0.06),(0.18,-0.06),(0.06,-0.006), \\
\\
(0.3,-0.18),(0.18,-0.18),(0.06,-0.18),(0.3,-0.3), \\
(0.18,-0.3),(0.06,-0.3),(-0.06,-0.06),(0.06,0.06)\},
\end{array}
$$

are tested and the one is chosen, that features the highest PSNR when employed in Algorithm 3.2.8.

It remains to consider a stopping criterion for Algorithm 3.2.8. We stop the iterative procedure of Algorithm 3.2.8 whenever either

$$
\frac{\left\|U^{(\tau)}-U^{(\tau-1)}\right\|}{\left\|U^{(\tau-1)}\right\|}<10^{-5},
$$

for some iterates $U^{(\tau)}, U^{(\tau-1)}$, or the number of iterations $\tau$ exceeds 500 . This has proven to lead to good results: All runs of Algorithm 3.2.8 with filters belonging to Set-Up 5.1.2 (classical) reached the threshold in (5.1.4) with $\tau<400$. Moreover, all runs of Algorithm 3.2.8 with filters belonging to Set-Up 5.1.3 (strongly) and 5.1.4 (weakly) that never reached the threshold in (5.1.4), seem to do so due to numerical instability, since when iterated for 10000 additional iterations no substantial change is observed. Notably, also weakly admissible filters employed in Algorithm 3.2.8 lead to a convergence.

The remaining parameters are fixed and listed in Table 5.3. Let us detail the protocol of the three experiments in the following. Note, that the protocols are given for a fixed covariance $\sigma^{2} \Xi$.

\section{Experiment 1:}

(i) $\mu$ is optimized for the given $U$ and $U+\varepsilon$ via Algorithm 5.1.6. 
(ii) Given $\mu$ from (i), $y_{1}, y_{2}$ and $r_{1}, r_{2}$ are optimized via grid-search as detailed in (5.1.2) and (5.1.3), respectively.

(iii) In Tables B.1, B.2 and B.3 in Appendix B the trained parameters are reported. In Tables 5.4, 5.5 and 5.6 the resulting maximal PSNR values are reported.

\section{Experiment 2:}

(i) The trained parameters of Experiment 1, cf. Tables B.1, B.2 and B.3 in Appendix B, are used to compute input filters for all Set-Ups.

(ii) The input filters, now fixed, are applied to the same images, now corrupted with newly drawn random matrices $\varepsilon_{k} \in \mathcal{N}\left(0, \sigma^{2} \mathfrak{S}\right)$, for $k=1,2, \ldots, 100$.

(iii) In Table 5.7 the mean and standard deviation of the resulting PSNR values are reported, as well as, how often Set-Up 5.1.3 (strongly) and 5.1.4 (weakly) outperformed Set-Up 5.1.2 (classical), respectively.

\section{Experiment 3:}

(i) On the 10 test images, each corrupted with a known realization of the chosen covariance, the parameter $\mu$ is trained via Algorithm 5.1.6. Using each of the so obtained $\mu$, the parameters $y_{1}, y_{2}, r_{1}, r_{2}$ are trained via grid search, as detailed in (5.1.2) and (5.1.3), respectively.

(ii) Take the mean of the 10 trained parameters, respectively, and compute filters for each of the Set-Ups. We report the mean and standard deviation of the trained parameters in Table 5.8 and the mean and standard deviation of the PSNR values on the training set in Table 5.9.

(iii) The input filters, now fixed, are applied to the test images $U_{k}$, corrupted with newly drawn random matrices $\varepsilon_{k} \in \mathcal{N}\left(0, \sigma^{2} \subseteq\right)$, for $k=1,2, \ldots, 100$.

(iv) In Table 5.10 the mean and standard deviation of the resulting PSNR values on the test set are reported, as well as, how often Set-Up 5.1.3 (strongly) and 5.1.4 (weakly) outperformed Set-Up 5.1.2 (classical), respectively.

\subsubsection{Discussion of the Results}

Considering Experiment 1 we find that the augmented models mostly outperform the classical model, however, they do so with a tight margin. Notably, the weakly admissible Set-Up 5.1.4 yields stronger improvements in PSNR than its strongly admissible counterpart Set-Up 5.1.3. Going into detail, except for three cases, we can find better 
performing input filters for Algorithm 3.2.8 within Set-Up 5.1.3 (strongly) and/or SetUp 5.1.4 (weakly) compared to Set-Up 5.1.2 (classical), cf. Tables 5.4, 5.5 and 5.6. Note, that this improvement is achieved not optimizing parameters $y_{1}, y_{2}$ and $r_{1}, r_{2}$, but taking the best of 20 guesses. That improvement in PSNR is only marginal can also be seen in the visual examples shown in (c) and (d) of Figures 5.3, 5.4 and 5.5. However, the visually low improvement is partially explainable, since the improvement seems to manifest itself in reducing loss-of contrast, as can be seen in (b) of Figures 5.3, 5.4 and 5.5. Moreover, stronger improvements of 0.1 PSNR (marked bold in Tables 5.4, 5.5 and 5.6) can be mostly observed for horizontally and vertically correlated noise (except for diagonal correlation in cameraman).

Experiment 2 shows that the results of Experiment 1 are stable under new realizations of the noise component. Comparing Tables 5.4, 5.5 and 5.6 with Table 5.7 we observe that all bold marked improvements of at least 0.1 PSNR over Set-Up 5.1.2 (classical) stay bold when tested on newly drawn $\varepsilon \sim \mathcal{N}\left(0, \sigma^{2} \subseteq\right)$ (There is even one new bold results). Furthermore, the ranking of the Set-Ups is preserved, except in two cases (boat $\sigma=150, \mathfrak{S}=\mathbb{E}$ and goldhill $\sigma=150, \mathfrak{S}=\mathfrak{E}$ ), where Set-Up 5.1.4 (weakly) achieves better mean PSNR than Set-Up 5.1.2 (classical), which was not the case for Experiment 1. Furthermore, every time when Set-Up 5.1.2 (classical) outperforms the other two Set-Ups in more than 5 cases, there PSNR values where already close to each other in Experiment 1.

Experiment 3 shows stability when trained parameters are applied to unknown images of similar type. We observe that improvement of PSNR is higher and more stable for correlated noise (in our experiment horizontal and vertical, i.e. $\mathfrak{S}=\mathfrak{C}_{Z_{x}} \mathfrak{C}_{Z_{x}}^{*}$ and $\mathfrak{S}=\mathfrak{C}_{Z_{y}} \mathfrak{C}_{Z_{y}}^{*}$ ). Improvements of PSNR are in three of six cases significant (over 95 runs outperform Set-Up 5.1.2 (classical)). Also in Experiment 2 and 3 among the augmented models Set-Up 5.1.4 (weakly) was in general performing better than Set-Up 5.1.3 (strongly). 


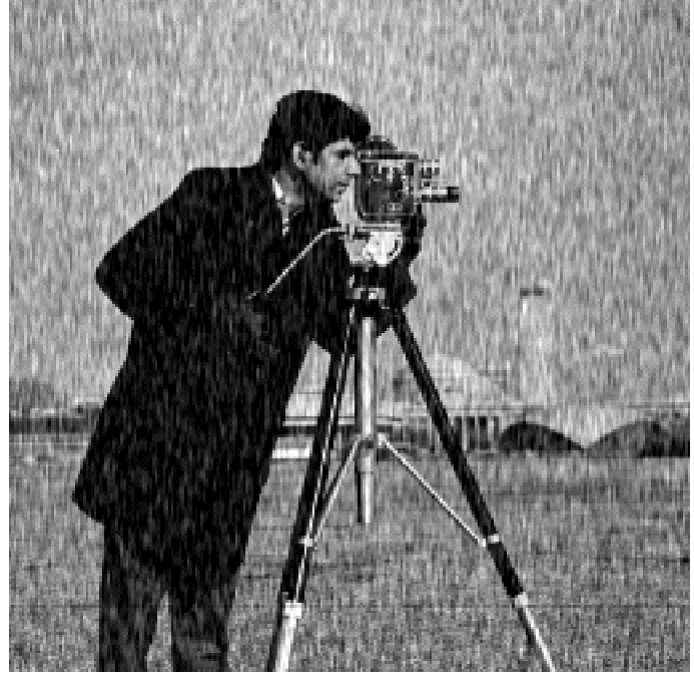

(a)

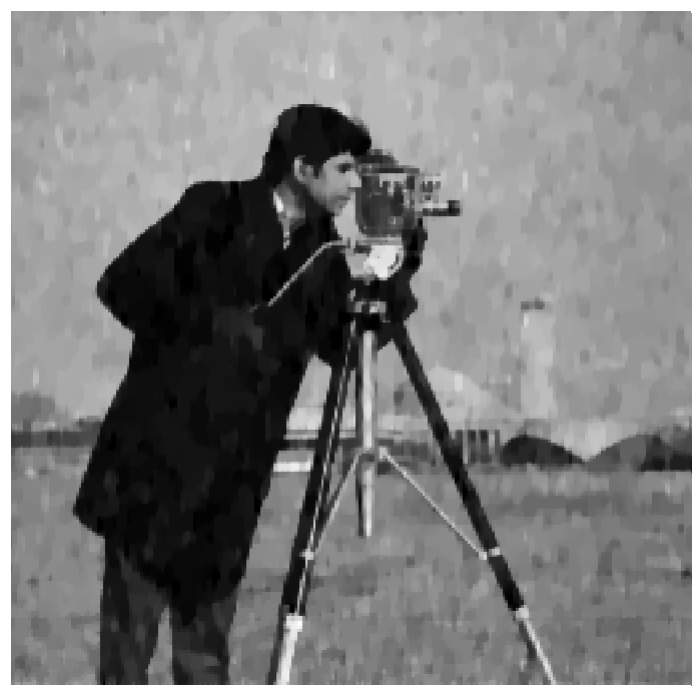

(c)

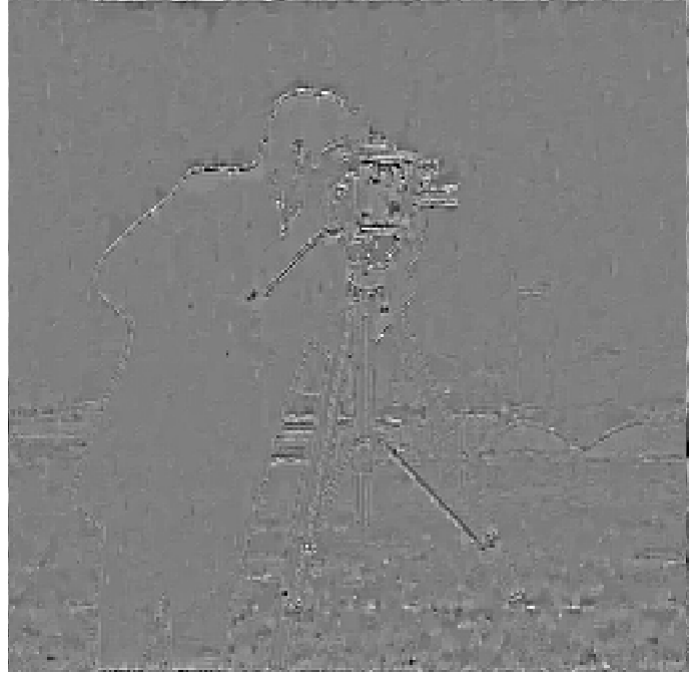

(b)

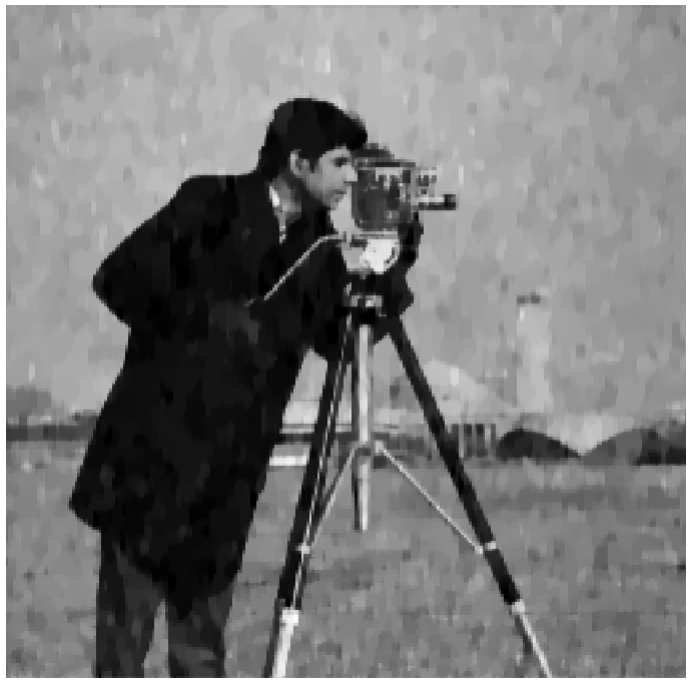

(d)

Figure 5.3: Cameraman corrupted with vertical noise $\left(\sigma=50, \mathfrak{S}=\mathfrak{C}_{Z_{y}} \mathfrak{C}_{Z_{y}^{*}}^{*}\right)$ in (a), its reconstructions $U_{1}^{\dagger}$ for Set-Up 5.1.2 (classical) (c) and $U_{2}^{\dagger}$ for Set-Up 5.1.3 (strongly) (d), as well as the difference $10 *\left(U_{1}^{\dagger}-U_{2}^{\dagger}\right)+127(\mathrm{~b})$. 


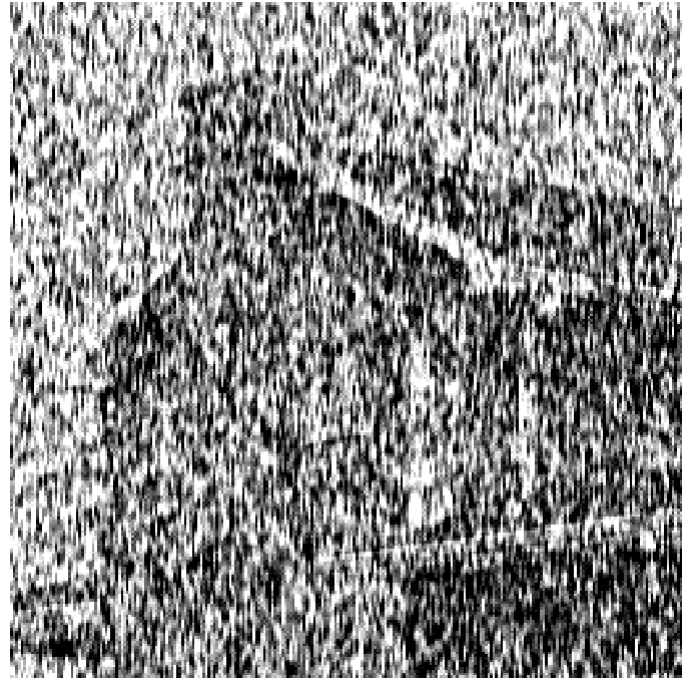

(a)

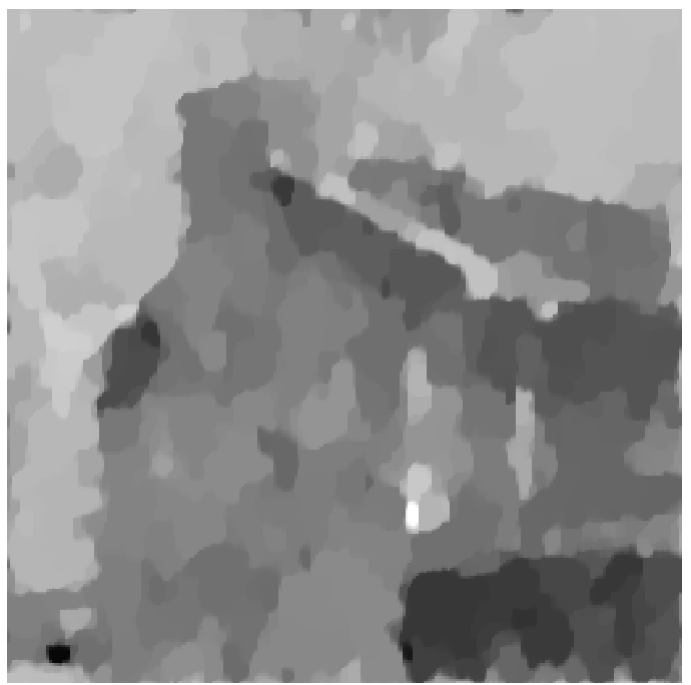

(c)

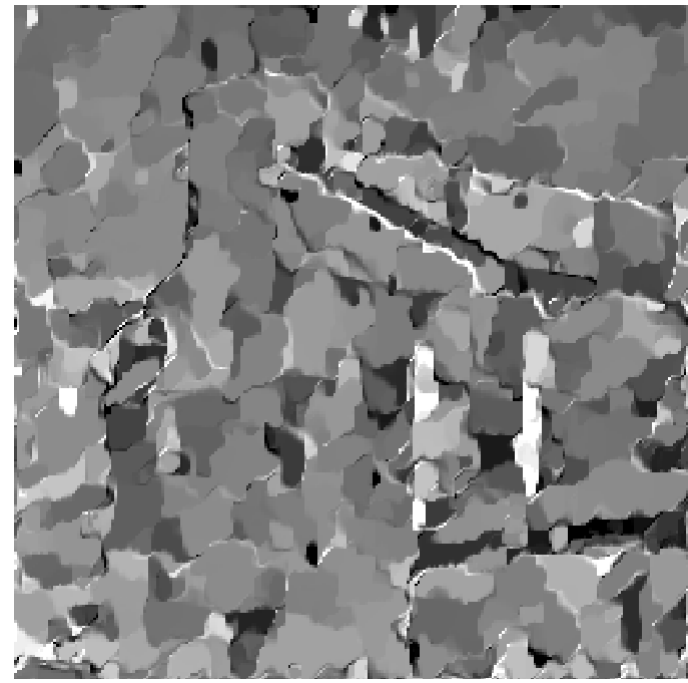

(b)

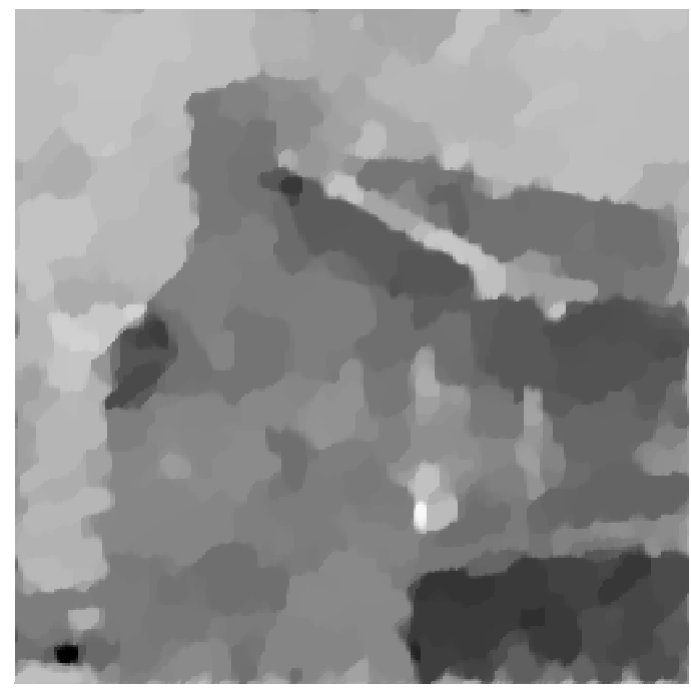

(d)

Figure 5.4: House corrupted by vertical noise $\left(\sigma=250, \mathfrak{S}=\mathfrak{C}_{Z_{y}} \mathfrak{C}_{Z_{y}}^{*}\right)$ in (a), its reconstructions $U_{1}^{\dagger}$ for Set-Up 5.1.2 (classical) (c) and $U_{2}^{\dagger}$ for Set-Up 5.1.4 (weakly) (d), as well as the difference $10 *\left(U_{1}^{\dagger}-U_{2}^{\dagger}\right)+127(\mathrm{~b})$. 


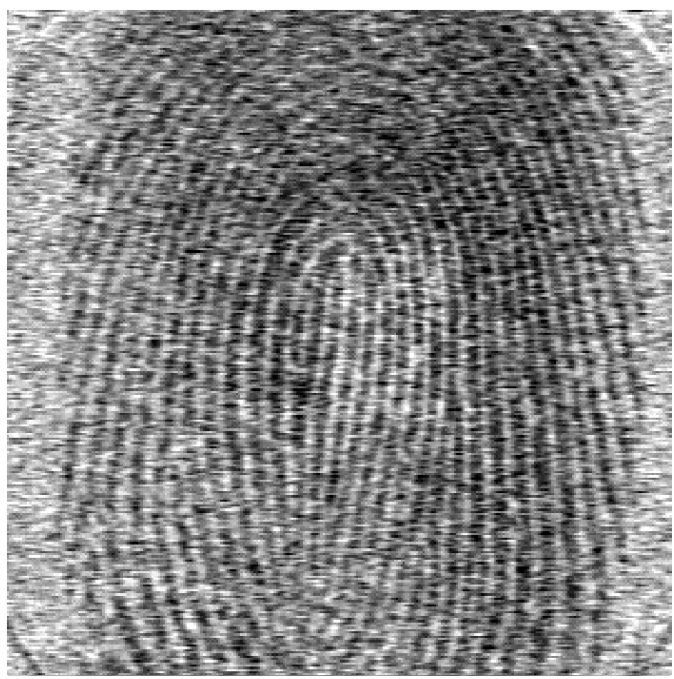

(a)

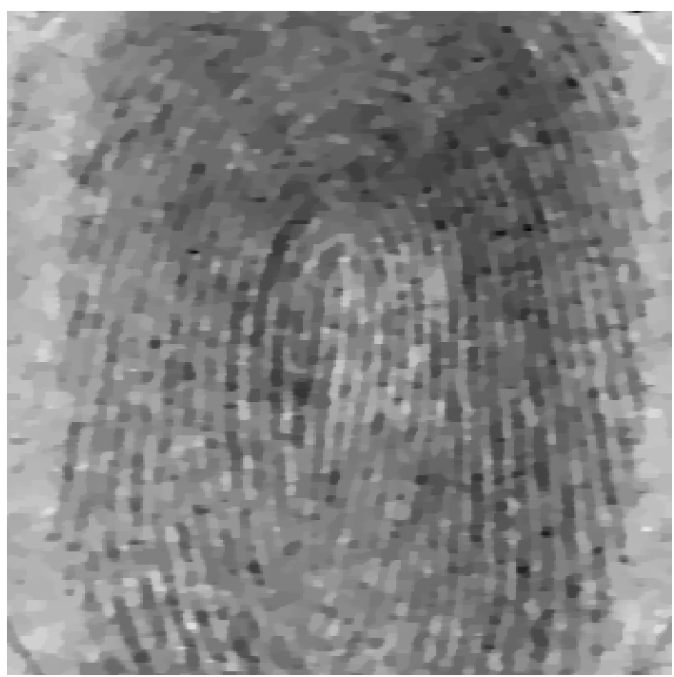

(c)

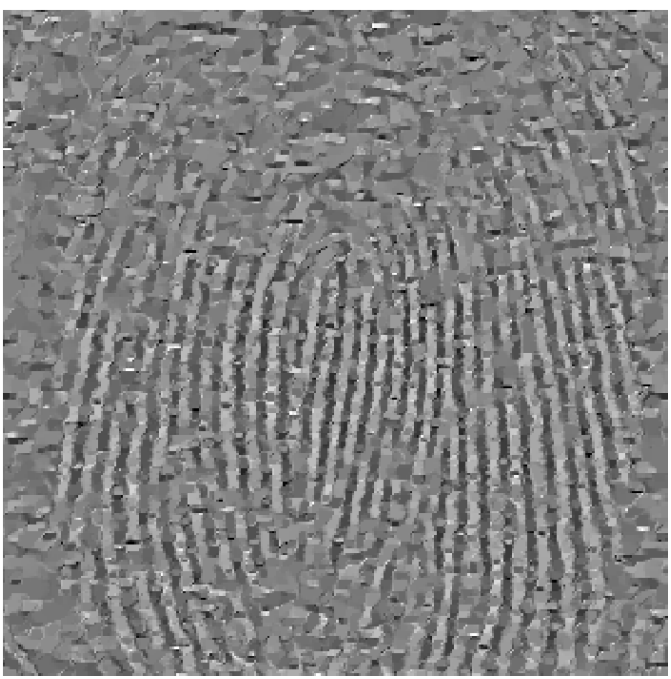

(b)

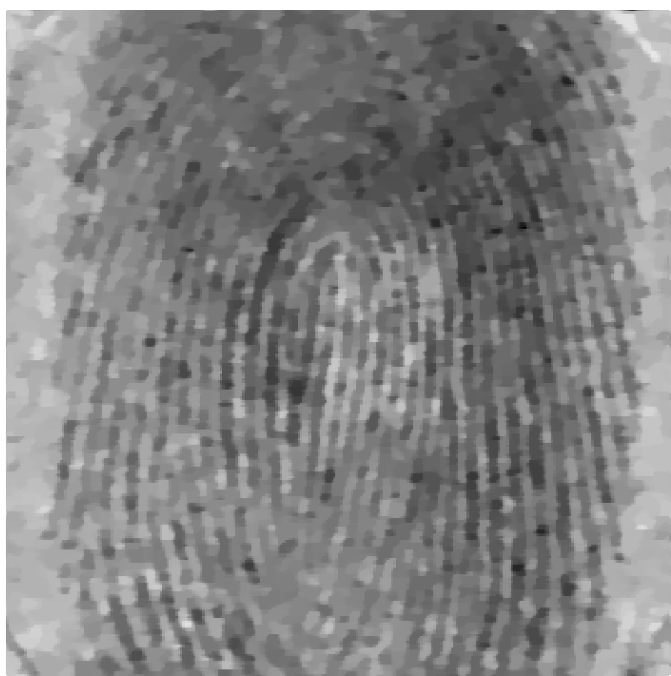

(d)

Figure 5.5: Fingerprint of individual 11 corrupted by horizontal noise $(\sigma=100, \subseteq=$ $\left.\mathfrak{C}_{Z_{x}} \mathfrak{C}_{Z_{x}}^{*}\right)$ in (a), its reconstructions $U_{1}^{\dagger}$ for Set-Up 5.1.2 (classical) (c) and $U_{2}^{\dagger}$ for SetUp 5.1.4 (weakly) (d), as well as the difference $10 *\left(U_{1}^{\dagger}-U_{2}^{\dagger}\right)+127$ (b). 
Table 5.4

Experiment 1 - Results 1/3 (The best PSNR is underlined, while each PSNR of Set-Up 5.1.3 or 5.1.4 that exceedes the PSNR of Set-Up 5.1.2 by more that 0.1 is marked bold, as a baseline the PSNR of the corrupted image $F$ is reported under

"Noisy").

\begin{tabular}{|c|c|c|c|c|c|c|}
\hline \multirow[t]{2}{*}{ Image } & \multirow[t]{2}{*}{$\sigma$} & \multirow[t]{2}{*}{ Covariance } & \multicolumn{4}{|c|}{ PSNR } \\
\hline & & & Noisy & $\begin{array}{c}\text { Set-Up } 1 \\
\text { (classical) }\end{array}$ & $\begin{array}{c}\text { Set-Up } 2 \\
\text { (strongly) }\end{array}$ & $\begin{array}{l}\text { Set-Up } 3 \\
\text { (weakly) }\end{array}$ \\
\hline \multirow{12}{*}{ barbara } & 50 & $\mathfrak{E}$ & 14.1428 & 23.2541 & 23.2794 & 23.3219 \\
\hline & 150 & E & 4.5817 & 20.8783 & 20.8819 & 20.8892 \\
\hline & 50 & $\mathfrak{C}_{Z_{x}} \mathfrak{C}_{Z_{x}}^{*}$ & 22.4255 & 25.1568 & 25.4647 & 25.5681 \\
\hline & 150 & $\mathfrak{C}_{Z_{x}} \mathfrak{C}_{Z_{x}}^{*}$ & 12.8347 & 21.2568 & 21.2695 & 21.3564 \\
\hline & 250 & $\mathfrak{C}_{Z_{x}}^{*} \mathfrak{C}_{Z_{x}}^{*}$ & 8.4416 & 19.8825 & 19.8989 & 20.0005 \\
\hline & 50 & $\mathfrak{C}_{Z_{y}} \mathfrak{C}_{Z_{y}}^{*}$ & 22.4387 & 25.0932 & 25.1468 & $\overline{25.1298}$ \\
\hline & 150 & $\mathfrak{C}_{Z_{y}} \mathfrak{C}_{Z_{y}}^{*}$ & 12.8739 & 21.2761 & 21.2845 & 21.2774 \\
\hline & 250 & $\mathfrak{C}_{Z_{y}} \mathfrak{C}_{Z_{v}}^{*}$ & 8.3884 & 19.8338 & 19.839 & 19.8378 \\
\hline & 50 & $\mathfrak{C}_{Z_{y}} \mathfrak{C}_{Z_{x}} \mathfrak{C}_{Z_{x}^{*}} \mathfrak{C}_{Z_{y}}^{*}$ & 28.6043 & 28.8761 & 28.9231 & 28.9033 \\
\hline & 150 & $\mathfrak{C}_{Z_{y}} \mathfrak{C}_{Z_{x}} \mathfrak{C}_{Z_{x}^{*}}^{*} \mathfrak{C}_{Z_{y}}^{*}$ & 19.1927 & 21.639 & 21.6454 & 21.6634 \\
\hline & 100 & $\mathfrak{c}_{Z_{d}} \mathfrak{C}_{Z_{d}}^{*}$ & 17.0956 & 22.5932 & 22.6328 & $\underline{22.6456}$ \\
\hline & 250 & $\mathfrak{C}_{Z_{d}} \mathfrak{C}_{Z_{d}}^{*}$ & 9.0809 & 20.0664 & 20.0715 & 20.1174 \\
\hline \multirow{12}{*}{ cameraman } & 50 & E & 14.1699 & 24.5280 & 24.5411 & 24.5755 \\
\hline & 150 & E & 4.5956 & 20.708 & 20.7284 & $\overline{20.7742}$ \\
\hline & 50 & $\mathfrak{C}_{Z_{x}} \mathfrak{C}_{Z_{x}}^{*}$ & 22.4004 & 26.8859 & 27.0914 & $\underline{27.1362}$ \\
\hline & 150 & $\mathfrak{C}_{Z_{x}}^{*} \mathfrak{C}_{Z_{x}}^{*}$ & 12.8172 & 21.4041 & 21.4964 & 21.5984 \\
\hline & 250 & $\mathfrak{C}_{Z_{x}} \mathfrak{C}_{Z_{x}}^{*}$ & 8.4231 & 19.7459 & 19.7895 & $\overline{19.882}$ \\
\hline & 50 & $\mathfrak{C}_{Z_{y}} \mathfrak{C}_{Z_{y}}^{*}$ & 22.4139 & 26.7970 & $\underline{26.9115}$ & 26.8822 \\
\hline & 150 & $\mathfrak{C}_{Z_{y}} \mathfrak{C}_{Z_{y}}^{*}$ & 12.8548 & 21.6384 & $\underline{21.6765}$ & 21.6393 \\
\hline & 250 & $\mathfrak{C}_{Z_{y}} \mathfrak{C}_{Z_{y}}^{*}$ & 8.4466 & 19.6093 & $\underline{19.625}$ & 19.6097 \\
\hline & 50 & $\mathfrak{c}_{Z_{y}} \mathfrak{c}_{Z_{x}} \mathfrak{c}_{Z_{x}^{*}} \mathfrak{C}_{Z_{y}}^{*}$ & 28.6644 & 29.3796 & 29.4618 & 29.3806 \\
\hline & 150 & $\mathfrak{C}_{Z_{y}} \mathfrak{C}_{Z_{x}} \mathfrak{C}_{Z_{x}^{*}}^{*} \mathfrak{C}_{Z_{y}}^{*}$ & 19.1405 & 22.4322 & $\underline{22.5876}$ & 22.5280 \\
\hline & 100 & $\mathfrak{C}_{Z_{d}} \mathfrak{C}_{Z_{d}}^{*}$ & 17.0677 & 23.8151 & 23.922 & $\underline{23.9231}$ \\
\hline & 250 & $\mathfrak{C}_{Z_{d}} \mathfrak{C}_{Z_{d}}^{*}$ & 9.1418 & 19.9304 & 19.9546 & 20.0511 \\
\hline \multirow{12}{*}{ fish } & 50 & E & 14.1275 & 22.417 & 22.419 & 22.4267 \\
\hline & 150 & E & 4.5992 & 18.4202 & 18.4173 & $\underline{18.4218}$ \\
\hline & 50 & $\mathfrak{C}_{Z_{x}} \mathfrak{C}_{Z_{x}}^{*}$ & 22.3258 & 25.3941 & 25.5271 & $\underline{25.6099}$ \\
\hline & 150 & $\mathfrak{C}_{Z_{x}} \mathfrak{C}_{Z_{x}}^{*}$ & 12.9177 & 19.1861 & 19.2443 & $\underline{19.3617}$ \\
\hline & 250 & $\mathfrak{C}_{Z_{x}} \mathfrak{C}_{Z_{x}}^{*}$ & 8.4182 & 17.6557 & 17.6705 & $\underline{17.7122}$ \\
\hline & 50 & $\mathfrak{C}_{Z_{y}}^{*} \mathfrak{C}_{Z_{y}}^{*}$ & 22.4312 & 25.4622 & 25.5591 & 25.6311 \\
\hline & 150 & $\mathfrak{C}_{Z_{y}} \mathfrak{C}_{Z_{y}}^{*}$ & 12.8863 & 19.2813 & 19.3238 & $\underline{19.3747}$ \\
\hline & 250 & $\mathfrak{C}_{Z_{y}} \mathfrak{C}_{Z_{y}}^{*}$ & 8.4635 & 17.5607 & 17.5733 & $\underline{17.605}$ \\
\hline & 50 & $\mathfrak{C}_{Z_{y}} \mathfrak{C}_{Z_{x}} \mathfrak{C}_{Z_{x}}^{*} \mathfrak{C}_{Z_{y}}^{*}$ & 28.666 & 29.0685 & $\underline{29.1012}$ & 29.0705 \\
\hline & 150 & $\mathfrak{C}_{Z_{y}} \mathfrak{C}_{Z_{x}} \mathfrak{C}_{Z_{x}^{*}}^{*} \mathfrak{C}_{Z_{y}}^{*}$ & 18.9893 & 20.5905 & $\underline{20.6601}$ & 20.6094 \\
\hline & 100 & $\mathfrak{C}_{Z_{d}} \mathfrak{C}_{Z_{d}}^{*}$ & 17.0833 & 21.9863 & 22.0057 & 22.0181 \\
\hline & 250 & $\mathfrak{C}_{Z_{d}} \mathfrak{C}_{Z_{d}^{*}}^{*}$ & 9.0931 & 17.6734 & 17.6792 & $\underline{17.6827}$ \\
\hline
\end{tabular}


Table 5.5

Experiment 1 - Results 2/3 (The best PSNR is underlined, while each PSNR of Set-Up 5.1.3 or 5.1.4 that exceedes the PSNR of Set-Up 5.1.2 by more that 0.1 is marked bold, as a baseline the PSNR of the corrupted image $F$ is reported under "Noisy")

\begin{tabular}{|c|c|c|c|c|c|c|}
\hline \multirow[t]{2}{*}{ Image } & \multirow[t]{2}{*}{$\sigma$} & \multirow[t]{2}{*}{ Covariance } & \multicolumn{4}{|c|}{ PSNR } \\
\hline & & & Noisy & $\begin{array}{c}\text { Set-Up } 1 \\
\text { (classical) }\end{array}$ & $\begin{array}{c}\text { Set-Up } 2 \\
\text { (strongly) }\end{array}$ & $\begin{array}{l}\text { Set-Up } 3 \\
\text { (weakly) }\end{array}$ \\
\hline \multirow{12}{*}{ boat } & 50 & E & 14.158 & 25.4112 & 25.4163 & $\underline{25.4493}$ \\
\hline & 150 & E & 4.6239 & 21.9357 & 21.9337 & $\overline{21.9330}$ \\
\hline & 50 & $\mathfrak{C}_{Z_{x}} \mathfrak{C}_{Z}^{*}$ & 22.4109 & $\overline{27.1046}$ & 27.2328 & $\mathbf{2 7 . 3 8 3 7}$ \\
\hline & 150 & $\mathfrak{c}_{Z_{x}} \mathfrak{C}_{Z_{x}}^{*}$ & 12.8296 & 22.4114 & 22.4532 & 22.5298 \\
\hline & 250 & $\mathfrak{C}_{Z_{x}} \mathfrak{C}_{Z_{x}}^{Z_{x}}$ & 8.4266 & 20.9462 & 20.9585 & $\overline{20.9506}$ \\
\hline & 50 & $\mathfrak{C}_{Z_{y}} \mathfrak{C}_{Z_{y}}^{*}$ & 22.3861 & 27.1273 & 27.1619 & 27.1542 \\
\hline & 150 & $\mathfrak{C}_{Z_{y}} \mathfrak{C}_{Z_{y}}^{*}$ & 12.8397 & 22.4649 & 22.4785 & 22.4701 \\
\hline & 250 & $\mathfrak{C}_{Z_{y}} \mathfrak{C}_{Z_{y}}^{*}$ & 8.3904 & 20.8315 & 20.8441 & $\underline{20.9009}$ \\
\hline & 50 & $\mathfrak{C}_{Z_{y}} \mathfrak{C}_{Z_{x}} \mathfrak{C}_{Z_{x}}^{*} \mathfrak{C}_{Z}^{*}$ & 28.7072 & 29.2868 & 29.322 & 29.3236 \\
\hline & 150 & $\mathfrak{C}_{Z_{y}} \mathfrak{C}_{Z_{x}} \mathfrak{C}_{Z}^{*} \mathfrak{C}_{Z}^{*}$ & 19.177 & 23.139 & 23.1863 & 23.1721 \\
\hline & 100 & $\mathfrak{C}_{Z_{d}} \mathfrak{C}_{Z_{d}}^{*}$ & 17.0836 & 24.413 & 24.4524 & 24.4825 \\
\hline & 250 & $\mathfrak{c}_{Z_{d}} \mathfrak{C}_{Z_{d}}^{*}$ & 9.1019 & 21.0186 & 21.0261 & 21.018 \\
\hline \multirow{12}{*}{ goldhill } & 50 & E & 14.1433 & 26.1191 & 26.1159 & 26.1196 \\
\hline & 150 & $\mathbb{E}$ & 4.55878 & 22.8433 & 22.8410 & $\overline{22.8426}$ \\
\hline & 50 & $\mathfrak{c}_{Z_{x}} \mathfrak{C}_{Z_{x}}^{*}$ & 22.3756 & 27.4187 & 27.4783 & $\underline{27.5104}$ \\
\hline & 150 & $\mathfrak{C}_{Z_{x}} \mathfrak{C}_{Z_{x}}^{*}$ & 12.8819 & 23.2752 & 23.2927 & 23.3048 \\
\hline & 250 & $\mathfrak{C}_{Z_{x}} \mathfrak{C}_{Z_{x}}^{*}$ & 8.4149 & 21.7274 & 21.7354 & $\overline{21.7354}$ \\
\hline & 50 & $\mathfrak{C}_{Z_{v}} \mathfrak{C}_{Z_{y}}^{*}$ & 22.3838 & 27.4242 & $\overline{27.4635}$ & 27.5427 \\
\hline & 150 & $\mathfrak{C}_{Z_{y}} \mathfrak{C}_{Z_{y}}^{*}$ & 12.8374 & 23.3171 & 23.3358 & 23.3674 \\
\hline & 250 & $\mathfrak{c}_{Z_{y}} \mathfrak{c}_{Z_{y}}^{*}$ & 8.4127 & 21.7365 & 21.7584 & 21.792 \\
\hline & 150 & $\mathfrak{C}_{Z_{y}} \mathfrak{C}_{Z_{x}} \mathfrak{C}_{Z_{x}^{*}}^{*} \mathfrak{C}_{Z}^{*}$ & 28.6802 & 29.2293 & 29.2591 & 29.2286 \\
\hline & 150 & $\mathfrak{C}_{Z_{y}} \mathfrak{C}_{Z_{x}} \mathfrak{C}_{Z_{x}^{*}}^{*} \mathfrak{C}_{Z}^{*}$ & 19.1075 & 23.8387 & $\underline{23.858}$ & 23.8391 \\
\hline & 100 & $\mathfrak{C}_{Z_{d}} \mathfrak{C}_{Z_{d}}^{*}$ & 17.0466 & 25.0705 & 25.0727 & 25.0705 \\
\hline & 250 & $\mathfrak{c}_{Z_{d}} \mathfrak{C}_{Z_{d}}^{*}$ & 9.1199 & 22.0312 & 22.0394 & 22.0354 \\
\hline
\end{tabular}


Table 5.6

Experiment 1 - Results 3/3 (The best PSNR is underlined, while each PSNR of Set-Up 5.1.3 or 5.1.4 that exceedes the PSNR of Set-Up 5.1.2 by more that 0.1 is marked bold, as a baseline the PSNR of the corrupted image $F$ is reported under

"Noisy")

\begin{tabular}{|c|c|c|c|c|c|c|}
\hline \multirow[t]{2}{*}{ Image } & \multirow[t]{2}{*}{$\sigma$} & \multirow[t]{2}{*}{ Covariance } & \multicolumn{4}{|c|}{ PSNR } \\
\hline & & & Noisy & $\begin{array}{c}\text { Set-Up } 1 \\
\text { (classical) }\end{array}$ & $\begin{array}{l}\text { Set-Up } 2 \\
\text { (strongly) }\end{array}$ & $\begin{array}{l}\text { Set-Up } 3 \\
\text { (weakly) }\end{array}$ \\
\hline \multirow{12}{*}{ house } & 50 & (E) & 14.1569 & 27.2452 & 27.2585 & 27.2655 \\
\hline & 150 & $\mathfrak{E}$ & 4.6313 & 22.5591 & 22.567 & 22.5629 \\
\hline & 50 & $\mathfrak{C}_{Z_{x}} \mathfrak{C}_{Z_{x}}^{*}$ & 22.4311 & 29.0754 & 29.1212 & 29.0936 \\
\hline & 150 & $\mathfrak{C}_{Z_{x}} \mathfrak{C}_{Z_{x}}^{*}$ & 12.8108 & 23.4065 & 23.4461 & 23.4185 \\
\hline & 250 & $\mathfrak{C}_{Z_{x}}^{*} \mathfrak{C}_{Z_{x}}^{*}$ & 8.4222 & 21.1247 & 21.1426 & 21.1322 \\
\hline & 50 & $\mathfrak{C}_{Z_{y}} \mathfrak{C}_{Z_{y}}^{*}$ & 22.4071 & 29.0578 & 29.1346 & $\underline{29.2565}$ \\
\hline & 150 & $\mathfrak{C}_{Z_{y}} \mathfrak{C}_{Z_{v}}^{*}$ & 12.8298 & 23.4955 & 23.5329 & 23.5776 \\
\hline & 250 & $\mathfrak{C}_{Z_{v}} \mathfrak{C}_{Z_{y}}^{*}$ & 8.3606 & 21.3119 & 21.3447 & 21.4529 \\
\hline & 50 & $\mathfrak{C}_{Z_{y}} \mathfrak{C}_{Z_{x}} \mathfrak{C}_{Z_{x}}^{*} \mathfrak{C}_{Z_{v}}^{*}$ & 28.6677 & 30.7471 & 30.7789 & 30.8004 \\
\hline & 150 & $\mathfrak{C}_{Z_{y}} \mathfrak{C}_{Z_{x}} \mathfrak{C}_{Z_{x}^{*}}^{*} \mathfrak{C}_{Z_{y}}^{*}$ & 18.9937 & 24.136 & $\underline{24.1738}$ & 24.1420 \\
\hline & 100 & $\mathfrak{c}_{Z_{d}} \mathfrak{C}_{Z_{d}}^{*}$ & 17.0491 & 26.0758 & 26.0764 & 26.0854 \\
\hline & 250 & $\mathfrak{C}_{Z_{d}} \mathfrak{C}_{Z_{d}}^{*}$ & 9.1195 & 21.4136 & 21.4155 & $\overline{21.4342}$ \\
\hline \multirow{12}{*}{ lung } & 50 & $\mathfrak{E}$ & 14.1555 & 25.2919 & 25.2896 & 25.3044 \\
\hline & 150 & $\mathfrak{E}$ & 4.5992 & 21.3677 & 21.3704 & 21.3810 \\
\hline & 50 & $\mathfrak{C}_{Z_{x}} \mathfrak{C}_{Z_{x}}^{*}$ & 22.4103 & 27.0948 & 27.1544 & 27.1567 \\
\hline & 150 & $\mathfrak{C}_{Z_{x}} \mathfrak{C}_{Z_{x}}^{*}$ & 12.903 & 22.4043 & 22.4238 & 22.4073 \\
\hline & 250 & $\mathfrak{C}_{Z_{x}} \mathfrak{C}_{Z_{x}}^{*}$ & 8.3466 & 20.3939 & 20.4043 & 20.395 \\
\hline & 50 & $\mathfrak{C}_{Z_{y}} \mathfrak{C}_{Z_{y}}^{*}$ & 22.4205 & 27.1256 & 27.1913 & $\underline{27.2889}$ \\
\hline & 150 & $\mathfrak{C}_{Z_{y}} \mathfrak{C}_{Z_{y}}^{*}$ & 12.8796 & 22.4802 & 22.5215 & $\underline{22.6301}$ \\
\hline & 250 & $\mathfrak{C}_{Z_{y}} \mathfrak{C}_{Z_{y}}^{*}$ & 8.3594 & 20.5059 & 20.5434 & $\underline{22.6322}$ \\
\hline & 50 & $\mathfrak{C}_{Z_{y}} \mathfrak{C}_{Z_{x}} \mathfrak{C}_{Z_{x}}^{*} \mathfrak{C}_{Z_{y}}^{*}$ & 28.7713 & 29.0533 & $\underline{29.0769}$ & 29.0644 \\
\hline & 150 & $\mathfrak{C}_{Z_{y}} \mathfrak{C}_{Z_{x}} \mathfrak{C}_{Z_{x}}^{*} \mathfrak{C}_{Z_{y}}^{*}$ & 19.1252 & 23.3606 & 23.3999 & 23.3825 \\
\hline & 100 & $\mathfrak{C}_{Z_{d}} \mathfrak{C}_{Z_{d}}^{*}$ & 17.0767 & 24.5795 & 24.5916 & $\underline{24.616}$ \\
\hline & 250 & $\mathfrak{c}_{Z_{d}} \mathfrak{c}_{Z_{d}}^{*}$ & 9.1365 & 20.8641 & 20.8807 & $\underline{20.9066}$ \\
\hline
\end{tabular}


Table 5.7

Experiment 2 - Results (Presented are mean and standard variation of the PSNR. The best mean PSNR is underlined, while each mean PSNR of Set-Up 5.1.3 or 5.1.4 that exceedes the mean PSNR of Set-Up 5.1.2 by more that 0.1 is marked bold. On the right hand side the number of runs in which Set-Up 5.1.3 and 5.1.4 outperformed

Set-Up 5.1.2 is reported.)

\begin{tabular}{|c|c|c|c|c|c|c|c|}
\hline \multirow[t]{2}{*}{ Image } & \multirow[t]{2}{*}{$\sigma$} & \multirow[t]{2}{*}{ Covariance } & \multicolumn{3}{|c|}{ PSNR } & \multicolumn{2}{|c|}{$\#(\geq$ Set-Up 1$)$} \\
\hline & & & $\begin{array}{c}\text { Set-Up } 1 \\
\text { (classical) }\end{array}$ & $\begin{array}{c}\text { Set-Up } 2 \\
\text { (strongly) }\end{array}$ & $\begin{array}{l}\text { Set-Up } 3 \\
\text { (weakly) }\end{array}$ & $\begin{array}{c}\text { Set-Up } 2 \\
\text { (strongly) }\end{array}$ & $\begin{array}{l}\text { Set-Up } 3 \\
\text { (weakly) }\end{array}$ \\
\hline \multirow{5}{*}{ barbara } & 50 & $\mathfrak{E}$ & $23.2614 \pm 0.0168$ & $23.2863 \pm 0.018$ & $\underline{23.3269 \pm 0.0188}$ & 100 & 100 \\
\hline & 150 & $\mathfrak{E}$ & $20.8678 \pm 0.0386$ & $20.8678 \pm 0.0387$ & $\underline{20.8887 \pm 0.0389}$ & 100 & 100 \\
\hline & 50 & $\mathfrak{C}_{Z_{x}} \mathfrak{C}_{Z_{x}}^{*}$ & $25.1158 \pm 0.0251$ & $25.4197 \pm 0.0266$ & $\underline{25.5282 \pm 0.0257}$ & 100 & 100 \\
\hline & 150 & $\mathfrak{C}_{Z_{y}} \mathfrak{C}_{Z_{y}}^{*}$ & $21.27 \pm 0.0391$ & $\underline{21.2789 \pm 0.0391}$ & $21.2721 \pm 0.0391$ & 100 & 84 \\
\hline & 100 & $\mathfrak{C}_{Z_{d}} \mathfrak{C}_{Z_{d}}^{*}$ & $22.5451 \pm 0.0262$ & $22.5836 \pm 0.0273$ & $22.6037 \pm 0.027$ & 100 & 100 \\
\hline \multirow{5}{*}{ cameraman } & 50 & $\mathfrak{E}$ & $24.5597 \pm 0.0581$ & $24.5811 \pm 0.0582$ & $\underline{24.6138 \pm 0.0593}$ & 100 & 100 \\
\hline & 150 & $\mathfrak{E}$ & $20.576 \pm 0.086$ & $20.5754 \pm 0.0859$ & $\underline{20.6171 \pm 0.0852}$ & 49 & 99 \\
\hline & 50 & $\mathfrak{c}_{Z_{x}} \mathfrak{E}_{Z_{x}}^{*}$ & $26.8547 \pm 0.0664$ & $27.0461 \pm 0.0582$ & $\underline{27.0957 \pm 0.0689}$ & 100 & 100 \\
\hline & 150 & $\mathfrak{E}_{Z_{y}} \mathfrak{C}_{Z_{y}}^{*}$ & $21.4289 \pm 0.096$ & $\underline{21.4624 \pm 0.0963}$ & $21.4308 \pm 0.0958$ & 100 & 66 \\
\hline & 100 & $\mathfrak{C}_{Z_{d}} \mathfrak{C}_{Z_{d}}^{*}$ & $23.7796 \pm 0.0938$ & $\underline{23.9054} \pm 0.0582$ & $23.9030 \pm 0.0944$ & 100 & 100 \\
\hline \multirow{5}{*}{ fish } & 50 & $\mathfrak{E}$ & $22.4827 \pm 0.0264$ & $22.4830 \pm 0.0265$ & $\underline{22.4919 \pm 0.0263}$ & 64 & 100 \\
\hline & 150 & $\mathfrak{E}$ & $18.3574 \pm 0.0373$ & $18.3561 \pm 0.0374$ & $\underline{18.3605 \pm 0.0378}$ & 23 & 95 \\
\hline & 50 & $\mathfrak{C}_{Z_{x}} \mathfrak{E}_{Z_{x}}^{*}$ & $25.4145 \pm 0.0386$ & $25.5503 \pm 0.0395$ & $\underline{25.666 \pm 0.0398}$ & 100 & 100 \\
\hline & 150 & $\mathfrak{C}_{Z_{y}} \mathfrak{E}_{Z_{y}}^{*}$ & $19.2089 \pm 0.0416$ & $19.2474 \pm 0.0421$ & $19.2919 \pm 0.0411$ & 100 & 100 \\
\hline & 100 & $\mathfrak{C}_{Z_{d}} \mathfrak{C}_{Z_{d}}^{*}$ & $21.9499 \pm 0.0455$ & $21.9681 \pm 0.0463$ & $\underline{21.9804 \pm 0.0459}$ & 100 & 100 \\
\hline \multirow{5}{*}{ boat } & 50 & $\mathfrak{E}$ & $25.4405 \pm 0.0273$ & $25.4452 \pm 0.0273$ & $\underline{25.4768 \pm 0.0272}$ & 100 & 100 \\
\hline & 150 & $\mathfrak{E}$ & $21.8940 \pm 0.0472$ & $21.8909 \pm 0.0472$ & $\underline{21.8956 \pm 0.0476}$ & 0 & 72 \\
\hline & 50 & $\mathfrak{C}_{Z_{x}} \mathfrak{C}_{Z_{x}}^{*}$ & $27.0946 \pm 0.0296$ & $27.2183 \pm 0.0273$ & $\underline{27.3681 \pm 0.0303}$ & 100 & 100 \\
\hline & 150 & $\mathfrak{C}_{Z_{y}} \mathfrak{C}_{Z_{y}}^{*}$ & $22.4637 \pm 0.0626$ & $22.4778 \pm 0.0625$ & $22.4703 \pm 0.0623$ & 100 & 99 \\
\hline & 100 & $\mathfrak{C}_{Z_{d}} \mathfrak{E}_{Z_{d}}^{*}$ & $24.4063 \pm 0.0404$ & $24.4483 \pm 0.0405$ & $\underline{24.4814 \pm 0.0406}$ & 100 & 100 \\
\hline \multirow{5}{*}{ goldhill } & 50 & $\mathfrak{E}$ & $26.1386 \pm 0.029$ & $26.1365 \pm 0.0288$ & $\underline{26.1389 \pm 0.029}$ & 0 & 58 \\
\hline & 150 & $\mathfrak{E}$ & $22.8347 \pm 0.055$ & $22.8312 \pm 0.055$ & $\underline{22.8355 \pm 0.0552}$ & 1 & 60 \\
\hline & 50 & $\mathfrak{C}_{Z_{x}} \mathfrak{C}_{Z_{x}}^{*}$ & $27.4352 \pm 0.0322$ & $27.4956 \pm 0.0326$ & $\underline{27.5305 \pm 0.0328}$ & 100 & 100 \\
\hline & 150 & $\mathfrak{C}_{Z_{y}} \mathfrak{C}_{Z_{y}}^{*}$ & $23.3481 \pm 0.0558$ & $23.367 \pm 0.0559$ & $\underline{23.3963 \pm 0.0534}$ & 100 & 100 \\
\hline & 100 & $\mathfrak{C}_{Z_{d}} \mathfrak{E}_{Z_{d}}^{*}$ & $25.0471 \pm 0.0484$ & $\underline{25.0492 \pm 0.0484}$ & $25.0467 \pm 0.0483$ & 91 & 40 \\
\hline \multirow{5}{*}{ house } & 50 & $\mathbb{E}$ & $27.2364 \pm 0.0722$ & $27.2415 \pm 0.0728$ & $27.2487 \pm 0.0728$ & 75 & 100 \\
\hline & 150 & $\mathfrak{E}$ & $22.5466 \pm 0.0722$ & $\underline{22.5674 \pm 0.01270}$ & $22.5502 \pm 0.01242$ & 95 & 69 \\
\hline & 50 & $\mathfrak{C}_{Z_{x}} \mathfrak{C}_{Z_{x}}^{*}$ & $29.0733 \pm 0.0891$ & $\underline{29.1216 \pm 0.0903}$ & $29.086 \pm 0.0905$ & 100 & 92 \\
\hline & 150 & $\mathfrak{C}_{Z_{y}} \mathfrak{C}_{Z_{y}}^{*}$ & $23.4728 \pm 0.1469$ & $23.5148 \pm 0.1480$ & $\underline{23.5864 \pm 0.1480}$ & 100 & 100 \\
\hline & 100 & $\mathfrak{c}_{Z_{d}} \mathfrak{C}_{Z_{d}}^{*}$ & $26.0802 \pm 0.1235$ & $26.0816 \pm 0.1231$ & $\underline{28.0928 \pm 0.1228}$ & 90 & 98 \\
\hline \multirow{5}{*}{ lung } & 50 & $\mathfrak{E}$ & $25.3116 \pm 0.0393$ & $25.3107 \pm 0.0393$ & $\underline{25.3212 \pm 0.0392}$ & 30 & 100 \\
\hline & 150 & $\mathfrak{E}$ & $21.4474 \pm 0.0733$ & $21.4504 \pm 0.0737$ & $\underline{21.4656 \pm 0.0740}$ & 92 & 100 \\
\hline & 50 & $\mathfrak{C}_{Z_{x}} \mathfrak{E}_{Z_{x}}^{*}$ & $27.0712 \pm 0.0397$ & $27.1317 \pm 0.0396$ & $\underline{27.1386 \pm 0.0404}$ & 100 & 100 \\
\hline & 150 & $\mathfrak{C}_{Z_{y}} \mathfrak{C}_{Z_{y}}^{*}$ & $22.4511 \pm 0.0791$ & $22.4937 \pm 0.0798$ & $\underline{22.6042 \pm 0.0832}$ & 100 & 100 \\
\hline & 100 & $\mathfrak{C}_{Z_{d}} \mathfrak{C}_{Z_{d}}^{*}$ & $24.5563 \pm 0.0627$ & $24.5689 \pm 0.0629$ & $\underline{24.5896 \pm 0.062}$ & 100 & 100 \\
\hline
\end{tabular}


Table 5.8

Experiment 3 - Trained Parameters (Presented are mean and standard variation of the trained parameters)

\begin{tabular}{|c|c|c|c|c|c|c|}
\hline \multirow[t]{2}{*}{$\sigma$} & \multirow[t]{2}{*}{ Covariance } & \multicolumn{5}{|c|}{ Paramaters } \\
\hline & & $\begin{array}{c}\mu \\
\text { (all) }\end{array}$ & $\begin{array}{c}y_{1} \\
\text { (weakly) }\end{array}$ & $\begin{array}{c}y_{2} \\
\text { (weakly) }\end{array}$ & $\begin{array}{c}r_{1} \\
\text { (strongly) }\end{array}$ & $\begin{array}{c}r_{2} \\
\text { (strongly) }\end{array}$ \\
\hline 100 & $\mathfrak{E}$ & $0.01128 \pm 0.00061$ & $-0.0084 \pm 0.021$ & $0.0224 \pm 0.0252$ & $0.9166 \pm 0.1261$ & $1.1407 \pm 0.17$ \\
\hline 100 & $\mathfrak{C}_{Z_{x}} \mathfrak{C}_{Z_{x}}^{*}$ & $0.02308 \pm 0.00256$ & $-0.07 \pm 0$ & $0.0336 \pm 0.0179$ & $0.7408 \pm 0$ & $1.3193 \pm 0.0611$ \\
\hline 100 & $\mathfrak{C}_{Z_{y}} \mathfrak{C}_{Z_{y}}^{*}$ & $0.02283 \pm 0.00237$ & $0.0112 \pm 0.0151$ & $-0.0588 \pm 0.0224$ & $1.1921 \pm 0.146$ & $0.8301 \pm 0.1783$ \\
\hline
\end{tabular}

Table 5.9

Experiment 3 - Results on the Training Set (Presented are mean and standard variation of the PSNR. The best mean PSNR is underlined, while each mean PSNR of Set-Up 5.1.3 or 5.1.4 that exceedes the mean PSNR of Set-Up 5.1.2 by more that 0.1 is marked bold)

\begin{tabular}{c|c|c|c|c}
$\sigma$ & Covariance & $\begin{array}{c}\text { Paramaters } \\
\text { Set-Up 1 } \\
\text { (classical) }\end{array}$ & $\begin{array}{c}\text { Set-Up 2 } \\
\text { (strongly) }\end{array}$ & $\begin{array}{c}\text { Set-Up 3 } \\
\text { (weakly) }\end{array}$ \\
\hline 100 & $\mathfrak{E}$ & $21.6176 \pm 1.0882$ & $21.624 \pm 1.0875$ & $\underline{21.6553 \pm 1.08}$ \\
100 & $\mathfrak{C}_{Z_{x}} \mathfrak{C}_{Z_{x}}^{*}$ & $22.5573 \pm 0.8205$ & $22.6003 \pm 0.8085$ & $\underline{\mathbf{2 2 . 7 7 1 1 \pm 0 . 7 7 3 3}}$ \\
100 & $\mathfrak{C}_{Z_{y}} \mathfrak{C}_{Z_{y}}^{*}$ & $22.5447 \pm 0.8494$ & $21.5672 \pm 0.849$ & $\underline{\mathbf{2 2 . 6 3 4 2 \pm 0 . 8 5 3 5}}$
\end{tabular}

Table 5.10

Experiment 3 - Results on the Test Set (Presented are mean and standard variation of the PSNR. The best mean PSNR is underlined, while each mean PSNR of Set-Up 5.1.3 or 5.1.4 that exceedes the mean PSNR of Set-Up 5.1.2 by more that 0.1 is marked bold

On the right hand side the number of runs in which Set-Up 5.1.3 and 5.1.4 outperformed Set-Up 5.1.2 is reported.)

\begin{tabular}{c|c|c|c|c||c|c}
$\sigma$ & Covariance & $\begin{array}{c}\text { PSNR } \\
\text { Set-Up 1 } \\
\text { (classical) }\end{array}$ & $\begin{array}{c}\text { Set-Up 2 } \\
\text { (strongly) }\end{array}$ & $\begin{array}{c}\text { Set-Up 3 } \\
\text { (weakly) }\end{array}$ & $\begin{array}{c}\#(\geq \text { Set-Up 1) } \\
\text { Set-Up 2 } \\
\text { (strongly) }\end{array}$ & $\begin{array}{c}\text { Set-Up 3 } \\
\text { (weakly) }\end{array}$ \\
\hline 100 & $\mathfrak{E}$ & $22.3222 \pm 1.0012$ & $\underline{22.3529 \pm 1.0332}$ & $22.3469 \pm 1.0234$ & 83 & 77 \\
100 & $\mathfrak{C}_{Z_{x}} \mathfrak{C}_{Z_{x}}^{*}$ & $23.0122 \pm 0.7408$ & $23.0589 \pm 0.7533$ & $\underline{\mathbf{2 3 . 2 0 9 6 \pm 0 . 7 4 4 1}}$ & 99 & 98 \\
100 & $\mathfrak{C}_{Z_{y}} \mathfrak{C}_{Z_{y}}^{*}$ & $23.0310 \pm 0.7427$ & $23.0505 \pm 0.7425$ & $\underline{\underline{23.1114 \pm 0.7805}}$ & 99 & 89
\end{tabular}




\subsection{Input Filters Based on Wavelet Frames}

In this chapter we consider the decomposition model

$$
F=U+V,
$$

where $V$ contains all small-scale oscillating patterns, i.e. texture and noise. To this end we compare visually the cartoon computed with wavelet frame based filters with cartoons of the $T V-\ell^{2}$-problem. The main intuition and first implementation for applying these specific wavelet frames in the context of Algorithm 3.2.8 were provided to the author by Duy H. Thai. Throughout this chapter all $T V-\ell^{2}$ examples are computed with $\kappa=2$ as it is common in the classical $T V-\ell^{2}$, while all examples of the proposed filters were computed with $\kappa=1$ and $\beta=1$.

\subsubsection{Idea}

Table 5.11

Parameters of the Wavelet Frame Construction, cf. Appendix C

\begin{tabular}{c|c|c} 
Parameter & Parameter Space & Description \\
\hline$\gamma$ & $\mathbb{R}_{+}$ & Exponent of the Laplacian \\
$N$ & $\mathbb{N}$ & Directions of the wavelet frames \\
$I$ & $\mathbb{N}$ & Lowest Scale of the wavelet frames
\end{tabular}

In [CDOS12] Cai et al. draw a connection between employing the discrete gradient $\underline{\mathfrak{c}}_{\underline{D}}$ in a variational problem $\left(T V-\ell^{2}\right)$ and doing the same with a Haar wavelet frame, the first order cardinal B-spline (Chui [Chu92][p.177ff]). Let us motivate the proposed filters of this chapter by drawing a similar connection. Consider the $T V-\ell^{2}$ input-filters introduced in Remark 5.1.1. Note, that $A_{\mathrm{TV}}$ gives rise to a localized, inverse Laplacian given for $\mu, \beta \in \mathbb{R}_{+}$in the frequency domain by

$$
\mathfrak{C}_{A_{\mathrm{TV}}}=\mu\left(\mu+\beta \underline{\mathfrak{C}}_{\underline{D}}^{*} \underline{\mathfrak{C}}_{\underline{D}}\right)^{-1} .
$$

The idea is to move from the discrete Laplace operator defined in the spatial domain to a fractional Laplace B-spline, defined in the frequency domain. The Laplacian B-spline we consider was introduced by Van de Ville et al. [VDVBU05][Eq.(10) and 
(11)] (building on Rabut [Rab92]), for $\gamma \in \mathbb{R}_{+}$by

$$
\widehat{\phi}(x, y):=\left(\frac{4\left(\sin ^{2}\left(\frac{x}{2}\right)+\sin ^{2}\left(\frac{y}{2}\right)\right)-\frac{8}{3}\left(\sin \left(\frac{x}{2}\right) \sin \left(\frac{y}{2}\right)\right)}{\left(x^{2}+y^{2}\right)}\right)^{\frac{\gamma}{2}} .
$$

We propose to use input filters $(A, \underline{B}, \underline{\widetilde{B}})$ for Algorithm 3.2.8 based on the wavelet frames constructed in [VDVBU05] via (5.2.1). Moreover, we additionally use the further extension of these wavelets to directional wavelets as proposed by Unser et al. [USVDV09], using the $N$-th Riesz transform $(N \in \mathbb{N}$ ) (consider also Unser and Van de Ville [UVDV10]). Note that, we obtain in this fashion both, biorthogonal Laplacian B-spline filters $\underline{B}_{\mathrm{bi}}$ and $\underline{\widetilde{B}}_{\mathrm{bi}}$, featuring only weakly admissible, and, orthogonal Laplacian $B$-spline filters $\underline{B}_{\text {orth }}$ and $\underline{\widetilde{B}}_{\text {orth }}$, satisfying $\underline{B}_{\text {orth }}=\underline{\widetilde{B}}_{\text {orth }}$, hence strong admissibility. We detail the exact construction of $A, \underline{B}_{\mathrm{bi}}, \underline{\widetilde{B}}_{\mathrm{bi}}, \underline{B}_{\text {orth }}$ and $\underline{\widetilde{B}}_{\text {orth }}$ in Appendix C. In Table 5.11 the parameters determining the structure of the wavelet basis are listed.

\subsubsection{Results}

In Figure 5.6 we compare the $T V-\ell^{2}$-problem (l.h.s) with employing the weakly admissible biorthogonal Laplacian B-spline filters in Algorithm 3.2.8 for different scales. For the latter one, we find that $(\gamma, I, N)=(1.2,3,3)$, depicted in $(\mathrm{d})$ of Figure 5.6, produces a good cartoon texture decomposition. In Figure 5.7 we see the advantage of using the biorthogonal Laplacian B-spline filters over the $T V-\ell^{2}$-model. While the $T V-\ell^{2}$-cartoon has trouble keeping the upper edge of the table (c) and removing all texture from the table cloth (e), Algorithm 3.2.8 with the proposed weakly admissible filters does a better job in both cases see (d) and (f) in Figure 5.7. Notably, this results relates directly to the motivational observation of Chapter 3.1.2. Furthermore, comparing the close ups of the r.h.s. of Figure 5.7 with the $T V-G$-problem presented in Aujol et al. [AGCO06][Figure 4] we observe the same benefit in favour of the proposed filters. Let us compare with the more recently proposed models by Buades and Lisani in [BL16b][Figure 6]. While they achieve both - keeping the upper edge of the table and removing the pattern of the table cloth - their results blur the edges more in comparison to the proposed weakly admissible filters.

When comparing the strongly admissible orthogonal counterpart of the weakly admissible biorthogonal B-spline Laplacian filters, they achieve similar results, cf. (a) and (b) of Figure 5.8, however the weakly admissible filters feature less artefacts and a slightly better contrast, see (c) to (f) of Figure 5.8. Note, that the reason we observe ringing in (c) and (d) of Figure 5.8, is due to defining the filters in the frequency domain, cf. 
Appendix C. However, the occurrence of this artefact is less strong when considering the weakly admissible filters compared to the strongly admissible ones.

Last, in Figure 5.9 we compare a range of parameter choices for the biorthogonal Laplcian B-spline filters. As discussed in the introduction, a "good" cartoon-residual decomposition often depends on the application it serves, hence, when interested in smoother results one might consider higher $N \in \mathbb{N}$ (see (b) in Figure 5.9) or higher $\gamma \in \mathbb{R}_{+}$(as depicted for varying parameters in (c) to (e) of Figure 5.9). 


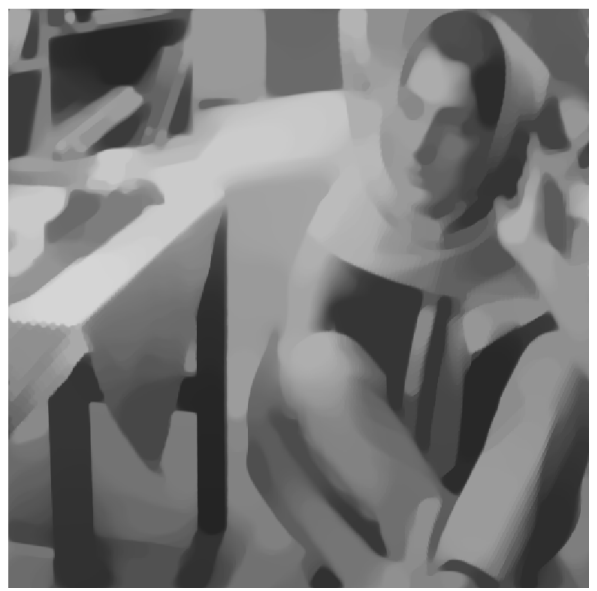

(a)

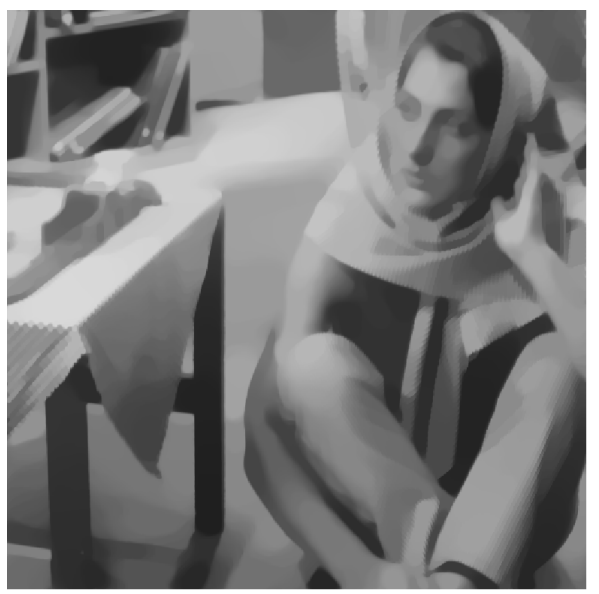

(c)

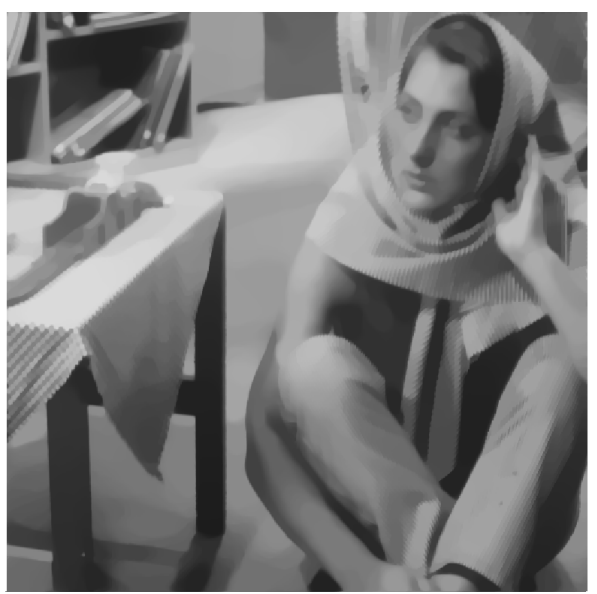

(e)

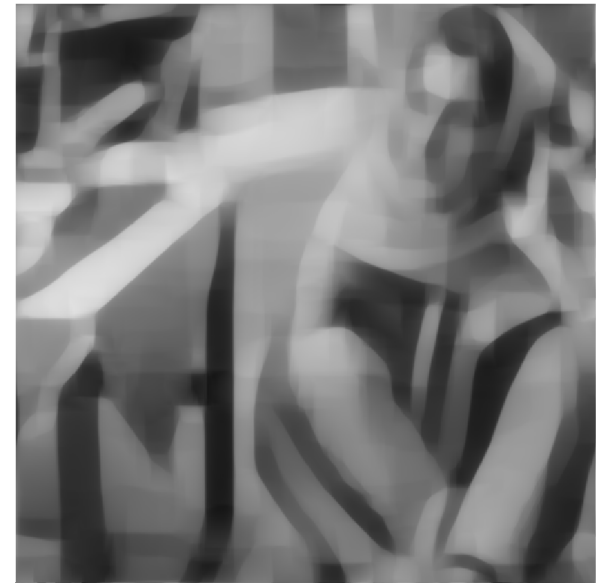

(b)

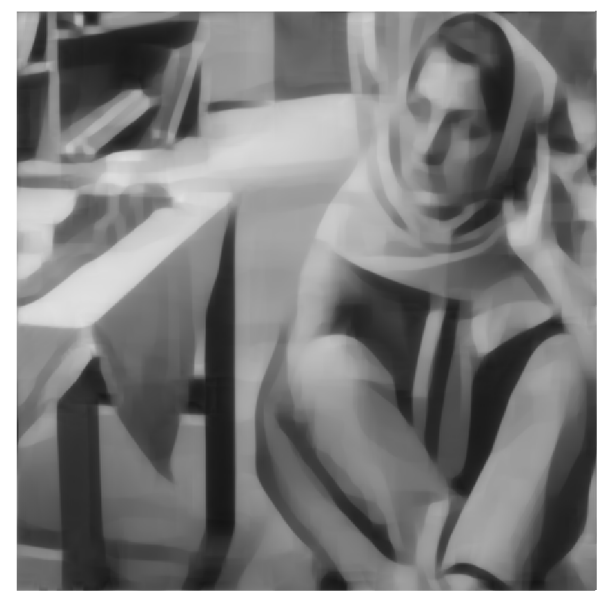

(d)

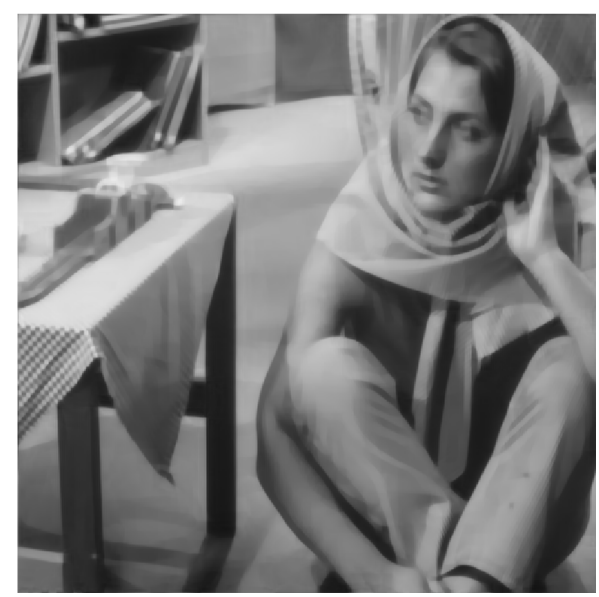

(f)

Figure 5.6: Comparison of the classical $T V-\ell^{2}$ (1.h.s.) and the weakly admissible biorthogonal Laplcian B-spline filters (r.h.s.). Separation of cartoon and texture is shown at different scales. For $T V-\ell^{2}$ parameter $\mu$ is set to 0.01 (a), 0.02 (c) and 0.03 (e). For the biorthogonal Laplacian B-splines the parameters $(\gamma, I, N)$ are set to $(1.2,4,3)(b),(1.2,3,3)(d)$ and $(1.2,2,3)(f)$, cf. Table 5.11 


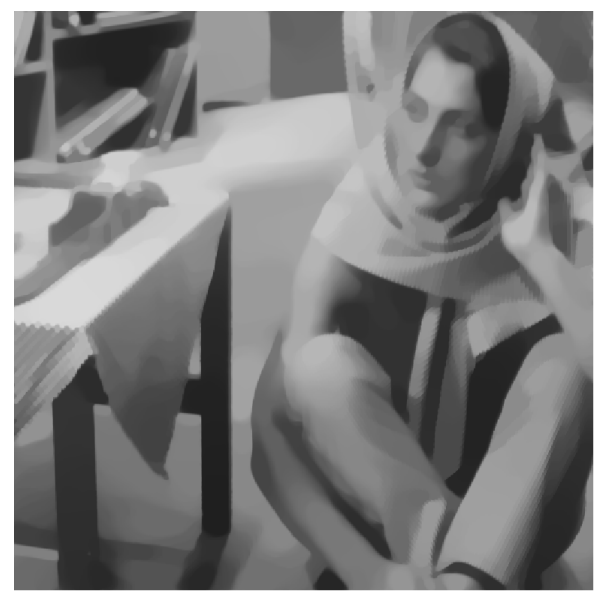

(a)

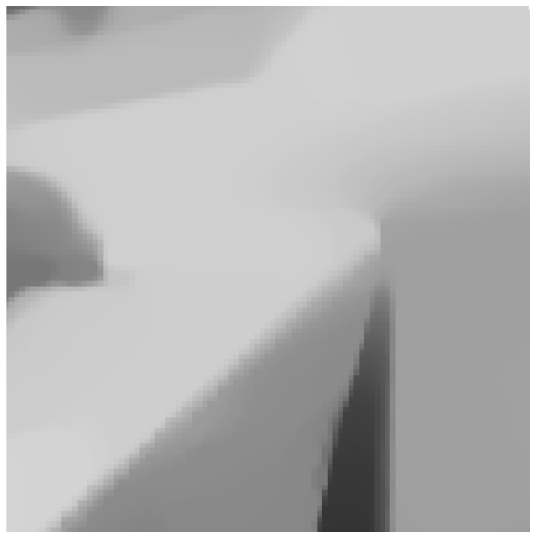

(c)

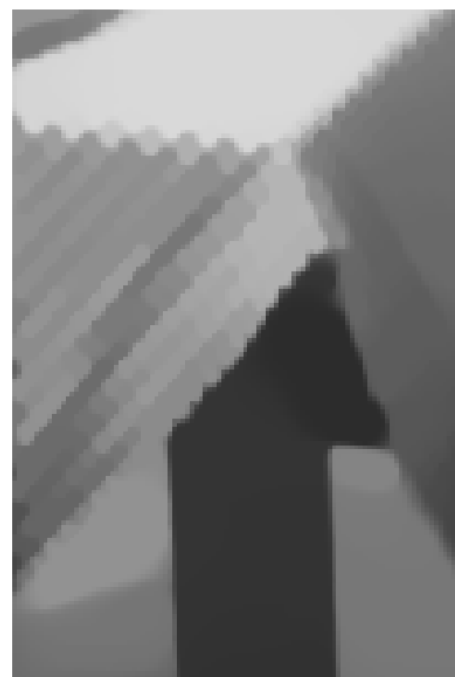

(e)

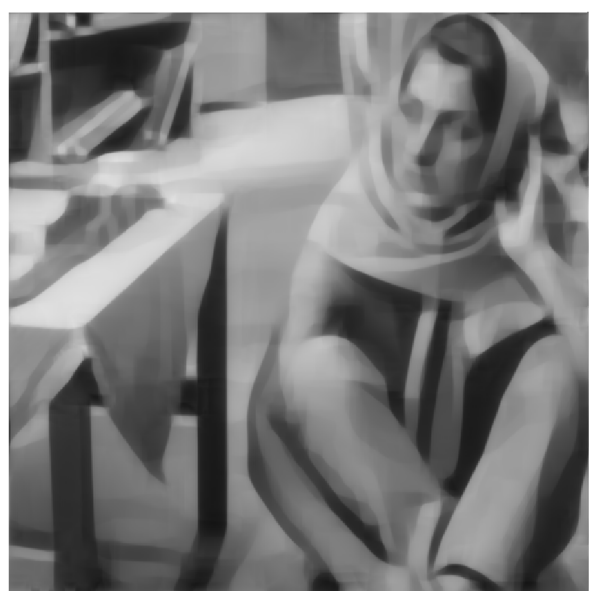

(b)

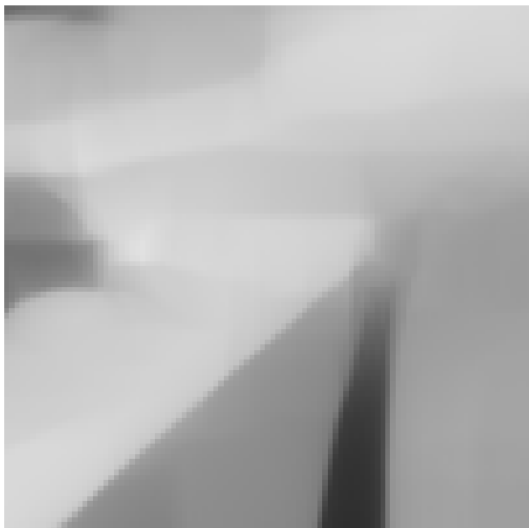

(d)

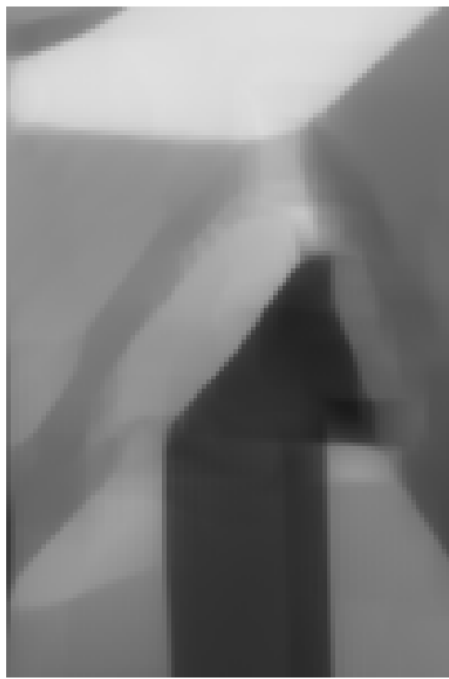

(f)

Figure 5.7: Close up for $T V-\ell^{2}$ with $\mu=0.02$ (a) and the biorthogonal Laplacian B-spline filters with $(\gamma, I, N)=(1.2,3,3)(\mathrm{b})$ : Upper table edge with $T V-\ell^{2}$ (c) and biorthogonal Laplacian B-spline filters (d) and table cloth with $T V-\ell^{2}(\mathrm{e})$ and biorthogonal Laplacian B-spline filters (f). 


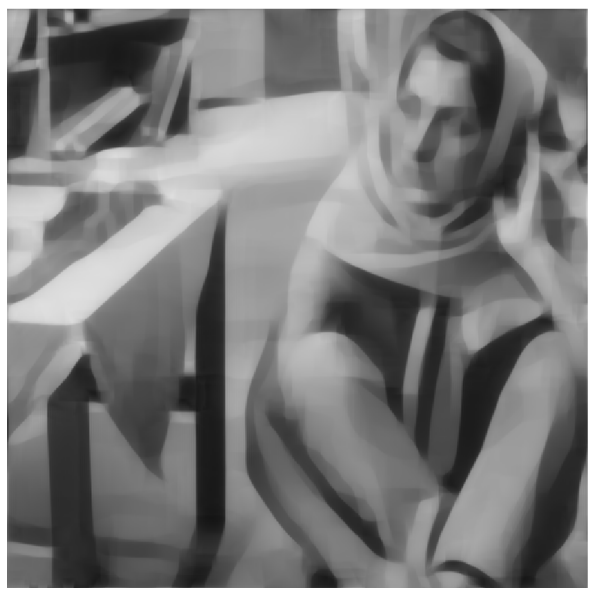

(a)

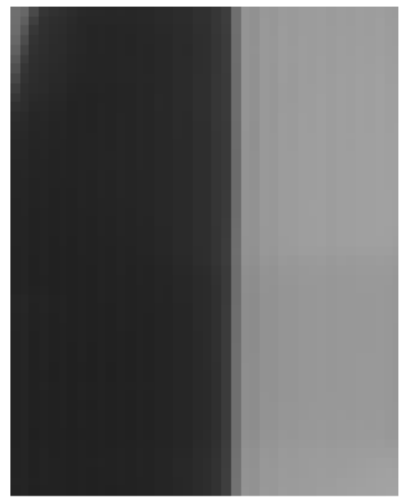

(c)

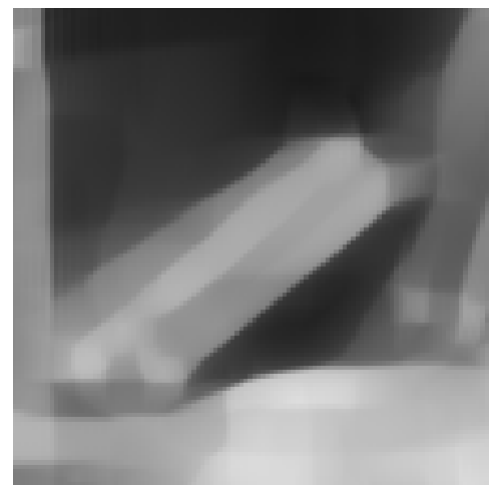

(e)

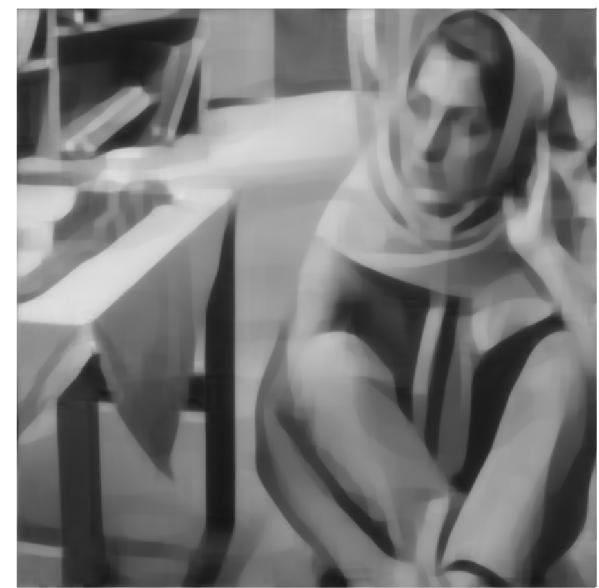

(b)

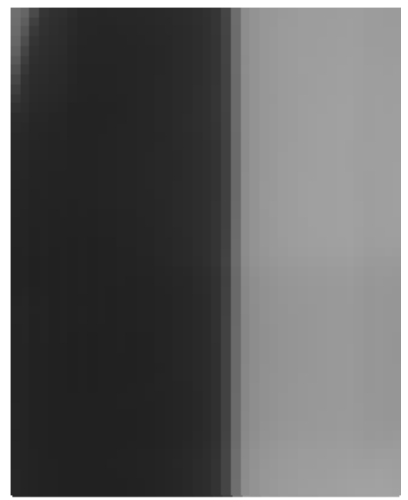

(d)

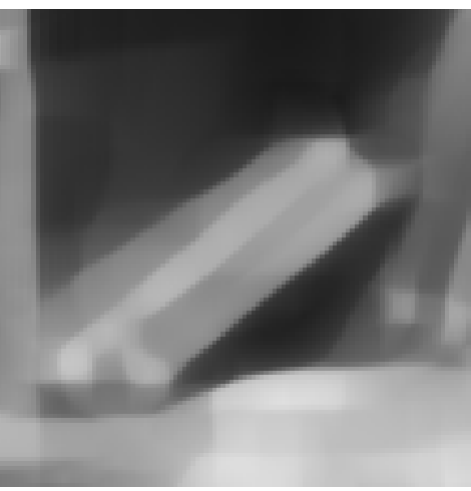

(f)

Figure 5.8: Comparison of the strongly admissible orthogonal Laplacian B-spline filters and the weakly admissible biorthogonal Laplacian B-spline filters for $(\gamma, I, N)=$ $(1.2,3,3)$. Close ups are shown of the ringing at the table leg (strongly admissible (c) and weakly admissible (d)) and keeping contrast at the books (strongly admissible (e) and weakly admissible (f)). 


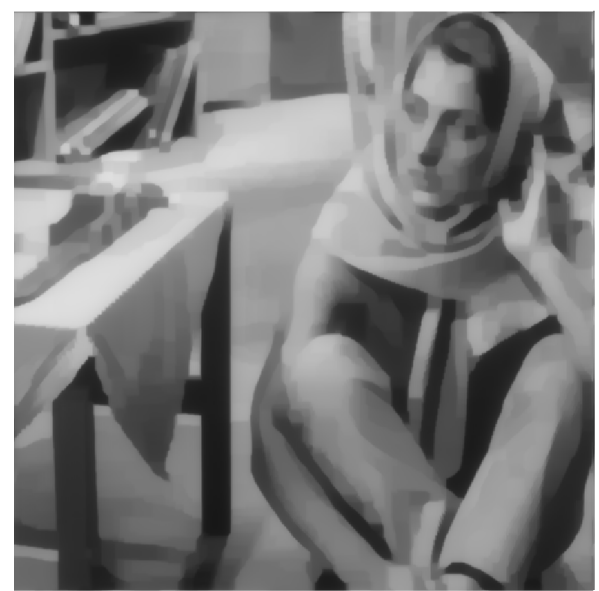

(a)

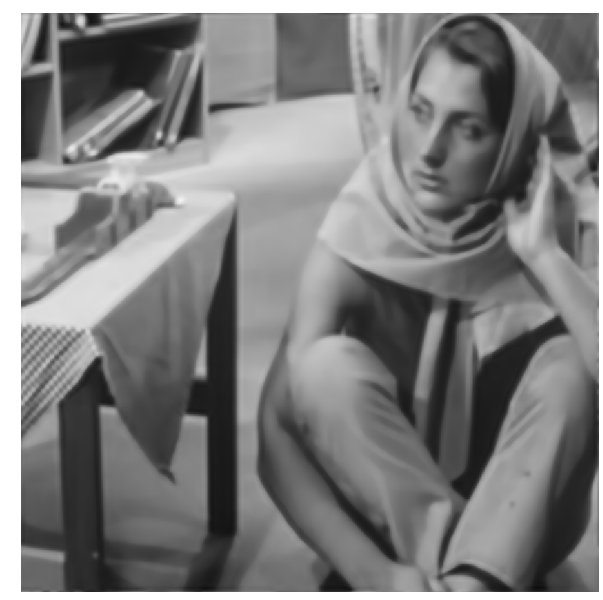

(c)

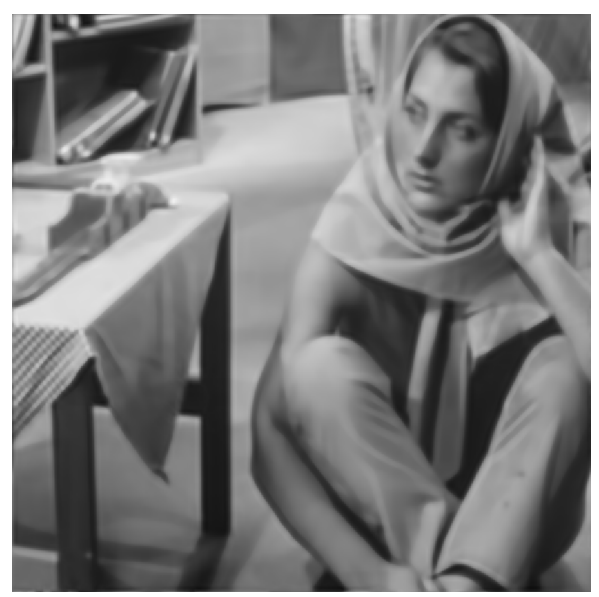

(e)

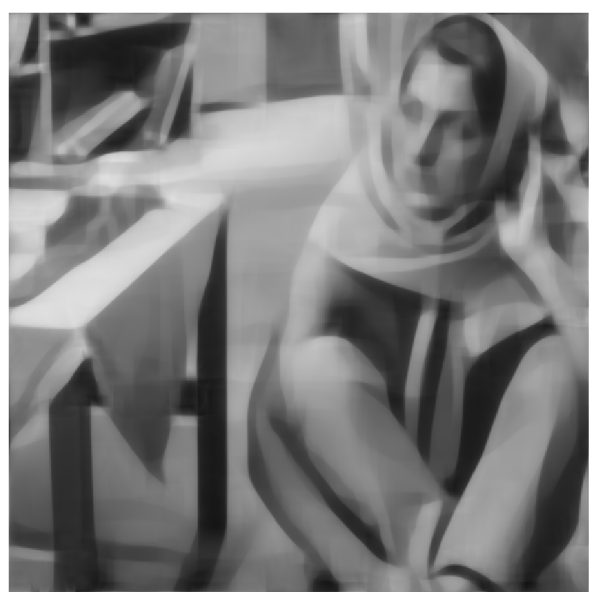

(b)

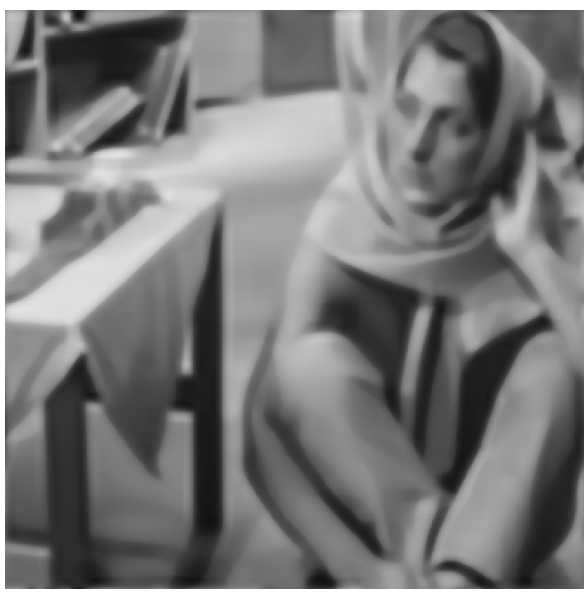

(d)

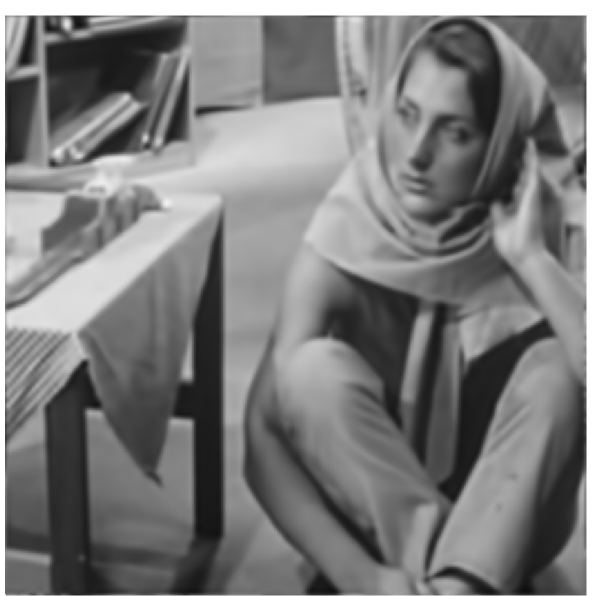

(f)

Figure 5.9: Further examples of the biorthogonal Laplacian B-spline with various choices of $(\gamma, I, N):(1.2,3,1)(\mathrm{a}),(1.2,3,5)(\mathrm{b}),(1.8,2,3)(\mathrm{c}),(1.8,3,3)(\mathrm{d}),(1.8,2,5)$ (e) and $(2.5,2,5)(\mathrm{f})$. 


\section{CHAPTER 6}

\section{Smudge Noise for Fingerprint Quality Estimation and its Validation}

In the following chapter a quality feature for fingerprint quality estimation is proposed, using the existing cartoon-texture-residual decomposition of Thai and Gottschlich [TG16], that was tailored for its use on fingerprint images. Moreover, a validation scheme for biometric quality estimators is proposed modelling the use-case of employment in comparison subsystems. Note, that chronologically this chapter should be the first in this thesis, since its development preceded the proposed Algorithm 3.2.8. Hence, this chapter is not an application of Algorithm 3.2.8, but rather a motivation for it. The cartoon-texture-residual decomposition of Thai and Gottschlich features an elaborate variational problem, including the discrete gradient and a curvelet transform of Candès et al. [CDDY06]. In the best case scenario training Algorithm 3.2.8 for smudge detection, could yield a faster and licence-free algorithm, solving a simpler variational problem, while achieving similar results. However, applying Algorithm 3.2.8 for this application is future work. This chapter, as well as, Chapter 7.2 have previously been published in Richter et al. $\left[\mathrm{RGM}^{+} 19\right]$ and are presented here with minor modifications. The idea for proposing the cartoon-texture-residual decomposition of [TG16] in fingerprint quality estimation arose in a working group of the SAMSI year long workshop on "Statistics and Applied Mathematics in Forensic Science", in which the author participated. Methodology, design and coding of the estimator, as well as, the validation scheme were worked out mainly by the author, profiting from fruitful discussions with the workgroup members who coauthor the paper.

In the following Chapter 6.1 an introduction to fingerprint quality estimation is given. Moreover, Chapter 6.1 introduces the rationale of the proposed quality feature "smudge", leading to the smudge noise quality estimator ( $\mathrm{SNoQE}$ ), and its validation, as well as, gives an overview over the existing literature. In Chapter 6.2 the cartoon-residual decomposition of Thai and Gottschlich is revisited and its outputs, that are utilized to set up SNoQE in Chapter 6.3. In particular, we emphasize on the parameters that need to be predefined, or tuned for the SNoQE algorithm, and possibly adjusted for different 
databases/sensors. In Chapter 6.4, we introduce an algorithmic validation scheme for assessing quality estimators, called robust biometric quality validation scheme (RBQ VS). Finally, in Chapter 6.5 we apply the RBQ VS and provide error-vs-reject characteristic (ERC) curves for the proposed SNoQE and the state-of-the-art quality estimator NFIQ 2.0 and its predecessor.

\subsection{Introduction}

There is an abundance of biometric features that are used in biometric identification and verification systems in commercial, governmental, and forensics applications. In all of these applications, there is a strong demand to optimize specific goals, typically a trade-off between security and usability over parameters such as false match / nonmatch rates and processing time. A direct method for optimization lies in improving comparison subsystems. This, however, can only lead to optimal results under the assumption that the processed biometric queries are of "sufficiently good quality". Thus, a second method does not touch such comparison subsystems, rather, it lowers error rates by disallowing biometric queries with not "sufficient quality" to partake in the comparison in the first place.

Following this second method requires considering the specific biometric features, a biometric comparison subsystem relies on. For instance in fingerprint analysis these are usually minutiae loci and their orientations, which are subject to various sources of errors, making quantifying a query's quality a highly non-trivial task.

Although there is, of course, a quantification of biometric verification and identification experiments, optimization of an automated comparison subsystem by quality estimation is not in itself a clearly-posed problem. Such subsystems are obviously prone to various algorithm-inherent errors, stemming, for example, from scanners, digitization and distortion. Thus there is a lack of quantification of the performance of quality estimators. In consequence two main challenges for this path to overcome are identified:

(a) Design quality features such that improved quality relates with improved false match / non-match rates, and

(b) design a validation scheme that, given a quality feature/estimator and a comparison subsystem, robustly quantifies the improvement of the error rates achieved by optimization with a quality threshold. 


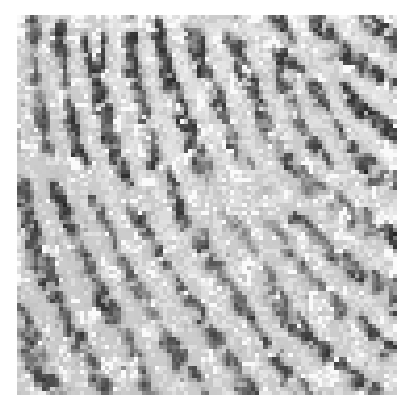

(a)

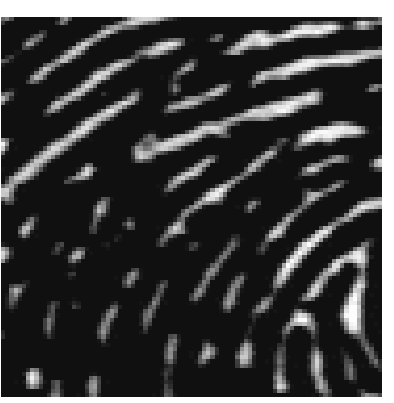

(b)

Figure 6.1: Examples how dry (a) or smudge (b) regions of a fingerprint obscure or even alter ridge patterns and minutiae.

\subsubsection{The Quality Feature "Smudge"}

Automated fingerprint comparison subsystems are usually based on comparing (marked) point clouds obtained from automated extraction of minutiae loci (and orientations) templates of fingerprints, which encode ridge bifurcations or ridge endings. A key factor that corrupts fingerprint images is the presence of large-scale noise, caused by too much pressure, called smudge, or too little pressure, called dryness, as both obscure existing minutiae or add spurious minutiae. At times smudge/dryness can lead to fingerprint's fringe pattern only partly visible, possibly resulting in wrongly detected ridge connections that obscure present ridge-endings or create spurious ridgebifurcations and vice versa, see Figure 6.1. For the two comparison subsystems used in this study, bozorth3 - based on mindtct - and the commercial "FingerCode 3" of the Matching SDK by Dermalog GmBH, it turns out, however, that mainly smudge noise relates to improved error rates. For this reason, only an implementation for a Smudge Noise Quality Estimator (SNoQE) is provided, while the ideas underlying both the SNoQE and the closely related Dryness Noise Quality Estimator (DNoQE) are detailed. An implementation of the latter can be obtained by straightforward adaptation of the former's.

Since smudge and dryness manifest itself as the absence of a clear oscillatory pattern, cartoon-residual decompositions can be used to assess in which pixel oscillatory pattern is observed and in which it is absent. The global three-part decomposition (G3PD) by Thai and Gottschlich [TG16] has been specifically modelled and trained for finding oscillatory pattern in order to find the fringe pattern of fingerprints in images. Their decomposition model computes a binary indicator for image regions that convey textureinformation, then additional morphological operations (cf. also [THG16]) are used to extract the region of interest ROI, the image region that contains the actual fingerprint. Here, the intermediate step of computing the indicator for texture-information, cf. Fig- 

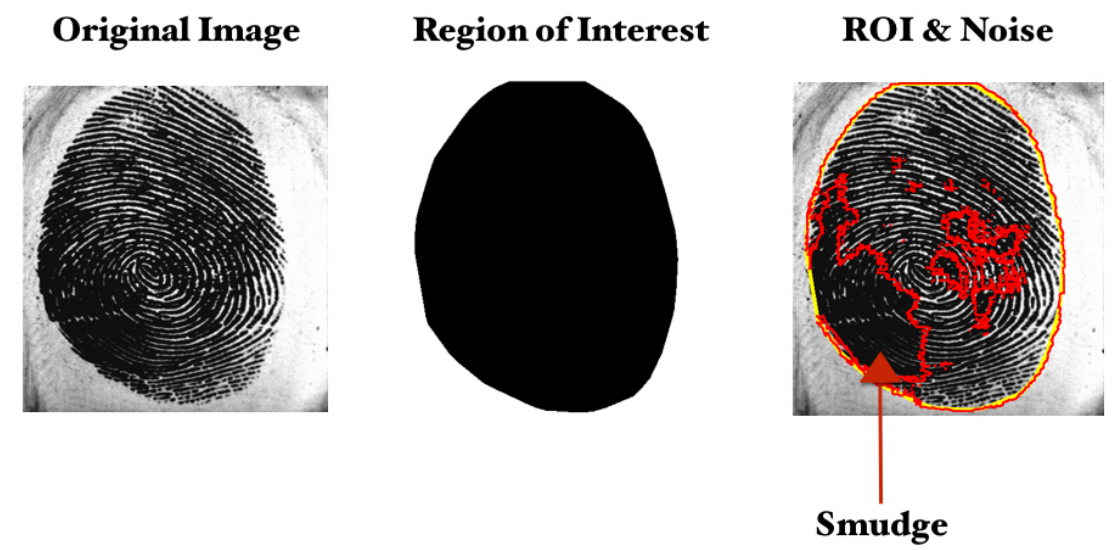

Figure 6.2: An original fingerprint image (left); Region of interest (ROI, center); and outline of ROI and regions of smudge within the ROI (right).

ure 6.2, is utilized in this chapter for smudge/dryness detection.

To obtain a quality feature, we first extract the region that conveys texture-information and the ROI according to [TG16]. Precisely those pixel correspond to smudge- or dryness-areas that are within the ROI, but convey no texture-information. In a second step the amount of smudge/dryness is assessed at pixels where texture, i.e. oscillation, is observed. More precisely, the amount of smudge or dryness is deduced by comparing at each such pixel the sum of grey-values in a neighbourhood with an expected lower/upper bound on that sum, governed by quantiles of the averaged inter-ridge distance distribution. By this heuristic estimation (not tuning) suitable parameters are obtained ensuring that minutiae are not mistaken for smudge, or dryness, respectively. It turns out that for the purpose of quality assessment based on rolled fingers, as well as optical, capacitive and thermal sensors, investigated here, smudge noise is decisive, dryness noise is less of interest.

\subsubsection{The Robust Biometric Quality Validation Scheme (RBQ VS)}

A validation scheme is proposed to robustly compare biometric quality estimators, as elaborated above, under the paradigm that excluding low quality imprints lowers comparison errors. For instance, one may want to train a quality threshold that robustly guarantees a lowered, at best, minimal equal error rate (EER) of a comparison subsystem. Indeed, the comparison scores of a specified comparison subsystem and the resulting error rates for each comparison threshold can be viewed as the ground truth for this evaluation. To this end, Lee et al. [LCCK08] separated a database into a training ( 80 out of 880 fingerprints) and a test set, trained a quality threshold minimizing the 
EER, thresholded the test set accordingly and compared the respective EERs of different quality estimators. While their scheme is intuitive, it turns out that it suffers from high variation under different separations into training and test sets, so that their single experiment lacks significance. Here, repeated random subsampling cross-validation is employed which allows many more runs (100 runs) and larger training and test sets, cf. Dubitzky et al.[DGB07, Sec.8.3]. This provides a necessary robustification, especially on small data sets.

\subsubsection{Literature on Validation Schemes}

To the best of the authors of $\left[\mathrm{RGM}^{+} 19\right]$ knowledge there is a lack of standardization for the performance evaluation of biometric query quality estimation. In the following some of the most prominent methods in the literature that have been used in the past are described (an overview over some can be found in Grother and Tabassi [GT07]). Several studies (Alonso-Fernandez et al. [AFFOG ${ }^{+}$07] Fierrez-Aguilar et al. [FACOGJ06], Olsen et al. [OXB12, OSB16], the latter involves 11 quality estimators and 3 comparison subsystems) assessing correlation of quality feature scores with each other and with comparison scores of genuine attempts yield mixed outcomes of various positive and negative correlations and no ranking of the quality features can be derived. Because comparison subsystems (should) implement expert human matching expertise, Shen et al. [SKK01] and Wu et al. [WXSL08] have compared quality estimators with human expert quality assignment. As long as variability of expert human assignment is not evaluated however, such studies have limited authority.

More closely related to our paradigm is the assessment of monotonicity of error rates in quality thresholds. While Fronthaler et al. [FKB06], Grother and Tabassi [GT07], Li et al. [LHF09] and Tao et al. [TYZ $\left.{ }^{+} 12\right]$ consider monotonicity only in single bins of quality, Alonso-Fernandez et al. [AFFOG ${ }^{+}$07], Tada et al. [TZM12], Xie et al. $\left[\mathrm{XYG}^{+} 12\right]$ and Phromsuthirak and Areekul [PA13] consider all bins above a given quality score and monotonicity therein. Moreover, Grother and Tabassi [GT07] and Yao et al. [YLBCR15] validate by comparing EERs due to random enrolment against best quality enrolment. Notably, as a non-ranking but multivalued feature giving detailed insight how employing the specified quality feature relates to lowered error rates, error versus reject characteristic (ERC) curves by Grother and Tabassi [GT07], plot false non-match rates against the fraction of genuine attempts rejected by the quality estimator (Here, an initial false non-match rate (often 0.1) yields a fixed comparison threshold). 


\subsubsection{Literature on Fingerprint Quality}

Other quality estimators aim at measuring general fingerprint features and their plausibility rather than directly measuring the above described kind of noise that impedes minutiae matching (overviews can be found in Alonso-Fernandez et al. [AFFOG ${ }^{+} 07$ ], Olsen et al. [OSB16] and Yao et al. [YLCR16]). Of the general quality features of fingerprints that have been proposed some typical ones are mentioned in the following. A prominent quality feature is directionality of local blocks, which is assessed via correlation matrices of the discrete gradient by Lim et al. [LJY02], and via the polartransformed Short-Time-Fourier-Transform by Phromsuthirak and Areekul [PA13]. The application of Gabor filters (Gottschlich [Got12]) for fingerprint quality estimation has been considered by Shen et al. [SKK01] and Olsen et al. [OXB12]. Apart from directionality, another quality feature that is often estimated is consistency, via differences of orientations of neighbouring blocks, via reduction to a 1-D structure and binarization by Lim et al. [LTS ${ }^{+}$04], or, more elaborately by Chen et al. [CJY04] in two dimensions. Alternatively, variances of grey-levels have been assessed by Joun et al. [JKCA03] in order to obtain clearness of the ridge-valley-structure. To this end, Fronthaler et al. [FKB06] computed responses of symmetry filters, and more recently Tao et al. [TYZ $\left.{ }^{+} 12\right]$ assessed principal components of local blocks. Additionally, Yoon et al. focus on image quality of latent fingerprints [YCLJ13], Teixeira and Leite [TL17] constructed recently a quality estimator for high resolution images and Alonso-Fernandez et al. [AFRM ${ }^{+} 08$ ] analyse the influence of fingerprint sensors on quality. With increased computational power, various quality features can be combined and machine learning techniques trained on suitable combinations, see Wu et al. [WXSL08], Li et al. [LHF09], Xie et al. [XYG $\left.{ }^{+} 12\right]$, Tada et al. [TZM12] and the NIST software [NFI15, NFI16]. Notably, many of these quality features often associate bad quality with rapid change of direction, which, unfortunately can also occur in perfect quality fingerprints, e.g. near singularities (cores, deltas and whorls) and arches, and sometimes also near minutiae.

The NIST Fingerprint Quality (NFIQ) is a highly popular, publicly available quality estimator, see [NFI15]. Its second version, NFIQ 2.0 [NFI16], has been designed using many of the above mentioned quality features, as well as an orientation field estimator from Kass and Witkin [KW87]. After a survey of 155 quality features from the literature, fourteen were selected and implemented to give a multi-dimensional feature vector. This feature vector was then used in a random forest binary classification that classified fingerprints into high and low utility. Assigning to each classified fingerprint the probability of being of high utility, multiplying with 100 and rounding to the closest integer yields the NFIQ 2.0 quality estimate. The random forest classifier has been trained on several large databases (in total 6629 images) for optical sensors. The NFIQ 
2.0 quality estimator has been validated by its ERC curves and by DET curves over the comparison threshold for three quality bins.

\subsection{The G3PD Model of Thai and Gottschlich}

\subsubsection{Cartoon-Texture-Residual Decomposition}

The global three-part decomposition (G3PD) introduced by Thai and Gottschlich [TG16] decomposes a given fingerprint image described by a real valued (grey-level) matrix $F \in \mathbb{R}^{n \times m}$ into cartoon $U$, texture $V$, and residual $\varepsilon$,

$$
F=U+V+\varepsilon
$$

For fingerprint images where $V$ takes the role of fringe patterns, Thai and Gottschlich adapted the minimization problem of Rudin et al. [ROF92] and its extensions by Meyer [Mey01] and Aujol and Chambolle [AC05] using the $T V$-norm for the cartoon, a fast computable $\ell^{1}$-curvelet-norm for the texture component (Candès et al. [CDDY06]) and penalizing the residual noise by a $\ell^{\infty}$-curvelet-norm of the same curvelet type. This leads to the objective function

$$
\mathcal{J}_{\mathrm{G} 3 \mathrm{PD}}(U, V):=\left|\underline{\mathfrak{C}}_{\underline{D}}(U)\right|_{1,1}+\mu_{1}|C(V)|_{1}+\mu_{2}|V|_{1},
$$

for $U, V \in \mathbb{R}^{n \times m}$ and $C(V)$ being the curvelet decomposition of $V$, to be minimized under the constraints

$$
\max _{(r, s, k, \ell) \in \mathcal{I}}\left|C_{r, s}\{\epsilon\}[k, \ell]\right| \leq \delta, \quad F=U+V+\varepsilon,
$$

where $\mathcal{I} \subset \mathbb{Z} \times \mathbb{Z} \times \mathbb{Z}^{2}$ is a suitable, pre-defined finite index set according to the curvelet transform of [CDDY06] and $\mu_{1}, \mu_{2}, \delta \in \mathbb{R}$. The constrained minimization is solved by an ADMM algorithm, and, as a further novelty, the parameter $\mu_{2}$ is updated in each iteration. The parameter $\mu_{1}$, as well as many more parameters, listed in Tab. 6.1, are trained beforehand for each database and remain fixed. In Tab. 6.2 we report their values from [TG16], trained for each of the FVC databases. For the NIST SD4, as the images are similar to those of FVC 2000 DB3, we took the same values. Notably, elaborate tuning seems not necessary.

\subsubsection{ROI and Fringe Pattern Extraction}

As a major advantage of the G3PD method, the texture component $V$ is exactly zero when locally no oscillation is detected (in that case the variation around $U$ is totally 
Table 6.1

Trainable parameters of the G3PD, detailed in [TG16, Tab.1]

\begin{tabular}{l|l}
\hline Description & Parameters \\
\hline Model parameters & $\mu_{1}, N$ \\
Algorithm parameters & $C, \beta_{1}, \beta_{2}, \beta_{3}, \gamma, p$ \\
Morphological parameters & $s, t, b$ \\
\hline
\end{tabular}

Table 6.2

Trained parameters for the G3PD, see [TG16, Sec.3.2])

\begin{tabular}{c|c|c|c|c|c|c|c}
\hline Database & $C$ & $\beta_{2}$ & $s$ & $t$ & $b$ & $p$ & $N$ \\
\hline FVC2000 DB1 & 0.045 & 0.0005 & 9 & 5 & 6 & 15 & 4 \\
FVC2000 DB2 & 0.045 & 0.01 & 9 & 5 & 6 & 15 & 4 \\
FVC2000 DB3 & 0.055 & 0.001 & 9 & 5 & 6 & 15 & 4 \\
FVC2002 DB1 & 0.02 & 0.001 & 9 & 5 & 6 & 15 & 4 \\
FVC2002 DB2 & 0.035 & 0.0005 & 9 & 5 & 6 & 15 & 4 \\
FVC2002 DB3 & 0.07 & 0.001 & 9 & 5 & 6 & 15 & 4 \\
FVC2004 DB1 & 0.015 & 0.1 & 9 & 5 & 6 & 15 & 4 \\
FVC2004 DB2 & 0.025 & 0.001 & 9 & 5 & 6 & 15 & 4 \\
FVC2004 DB3 & 0.035 & 0.001 & 9 & 5 & 6 & 15 & 4 \\
NIST SD4 & 0.055 & 0.001 & 9 & 5 & 6 & 15 & 4 \\
\hline
\end{tabular}

absorbed in $\varepsilon$ ). Thus, setting

$$
V_{\text {bin }}[k, \ell]=\left\{\begin{array}{l}
0 \text { if } V[k, \ell]=0 \\
1 \text { if } V[k, \ell] \neq 0
\end{array}\right\} \text { for all } 0 \leq k \leq n-1 \text { and } 0 \leq \ell \leq m-1
$$

gives an indicator for the existence of a local fringe pattern. As it may happen that small and isolated fringe-like patterns are also detected outside the fingerprint area, the following morphological operation, also from [TG16], introduces a new binary function that removes these. For parameters $s, t, b$ (cf. Tab. 6.1) consider for each pixel $\left[k_{0}, \ell_{0}\right]$ an $s \times s$-block centred at pixel $\left[k_{0}, \ell_{0}\right]$ along with 8 neighbouring blocks of the same size, and determine for each of the 9 blocks whether the number of pixels with $V_{\mathrm{bin}}[k, \ell]=1$ exceeds $\frac{s^{2}}{t}$. If at least $b$ of the 9 blocks exceed this threshold this pixel is marked as contributing to the fringe pattern, set

$$
V_{\text {fringe }}\left[k_{0}, \ell_{0}\right]=1 \text {, else set } V_{\text {fringe }}\left[k_{0}, \ell_{0}\right]=0
$$

In particular, the convex hull of $V_{\text {fringe }}[k, \ell]=1$ gives the binary ROI matrix, denoted by $I_{\text {ROI. }}$. 


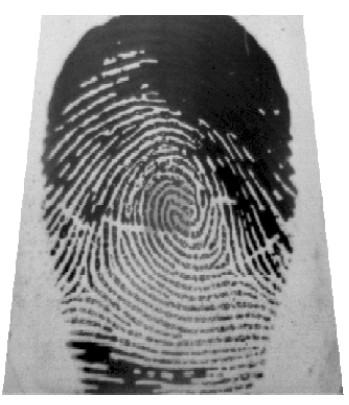

(a)

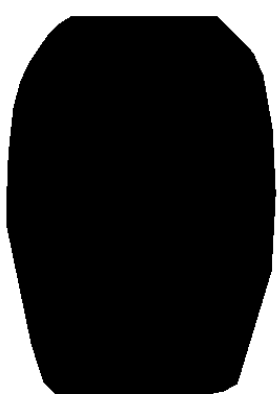

(b)

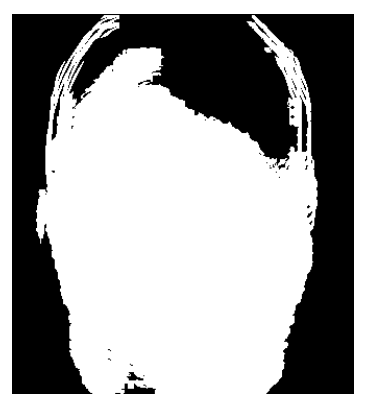

(c)

Figure 6.3: Example fingerprint (a), its inverted ROI $E-I_{\mathrm{ROI}}$ (where $E$ is the identity matrix) (b) and $V_{\text {fringe }}$ (c) (FVC 2004 DB2, finger 1, replica 8).

\subsection{The Smudge Noise Quality Estimator (SNoQE)}

Assume that all fingerprint images are given by matrices $F$ with entries taking greylevel values in $\Omega:=\{0,1,2, \ldots, 255\}$. Here 0 stands for black and 255 for white. As described in the previous chapter, after application of the G3PD we obtain the two binary matrices $I_{\mathrm{ROI}}$ and $V_{\text {fringe. }}$. As the central rationale of SNoQE the fringe pattern in $F$, encoded by $V_{\text {fringe }}$, must exhaust the full grey-scale spectrum in the ROI. This is achieved by the following histogram spreading pre-processing step.

\subsubsection{Contrast Enhancement}

For every single fingerprint image, denote by $c_{\text {black }}$ and $255-c_{\text {white }}$ the 0.05 and 0.95 quantiles of the empirical grey value distribution over the ROI, i.e. $c_{\text {black }} \in \Omega$ is the minimal grey value such that at least $5 \%$ of all ROI pixels attain grey levels in $\left\{0,1, \ldots, c_{\text {black }}\right\}$ and $c_{\text {white }} \in \Omega$ is the minimal value such that at least $5 \%$ of all ROI pixels attain grey values in $\left\{255-c_{\text {white }}, \ldots, 255\right\}$. Then for $0 \leq k \leq n-1$ and $0 \leq \ell \leq m-1$ the contrast enhanced image is given by

$$
F_{\mathrm{CE}}[k, \ell]:=255 \min \left\{\frac{\max \left\{F[k, \ell]-c_{\text {black }}, 0\right\}}{255-c_{\text {white }}-c_{\text {black }}}, 1\right\} .
$$

After this pre-processing we have in particular that $F_{\mathrm{CE}}$ is close to 255 for both valley and dry areas and close to 0 for ridge and smudge areas.

\subsubsection{Smudge Noise Estimation}

The SNoQE algorithm calculates a smudge component $F_{\mathrm{SNoQE}}$ of $F_{\mathrm{CE}}$, and the SNoQE score $q_{\mathrm{SNoQE}}$ is then given by the mean of grey-values of $F_{\mathrm{SNoQE}}$ within the ROI. For this purpose, the algorithm differentiates between pixels of two types in the ROI. Pixels 


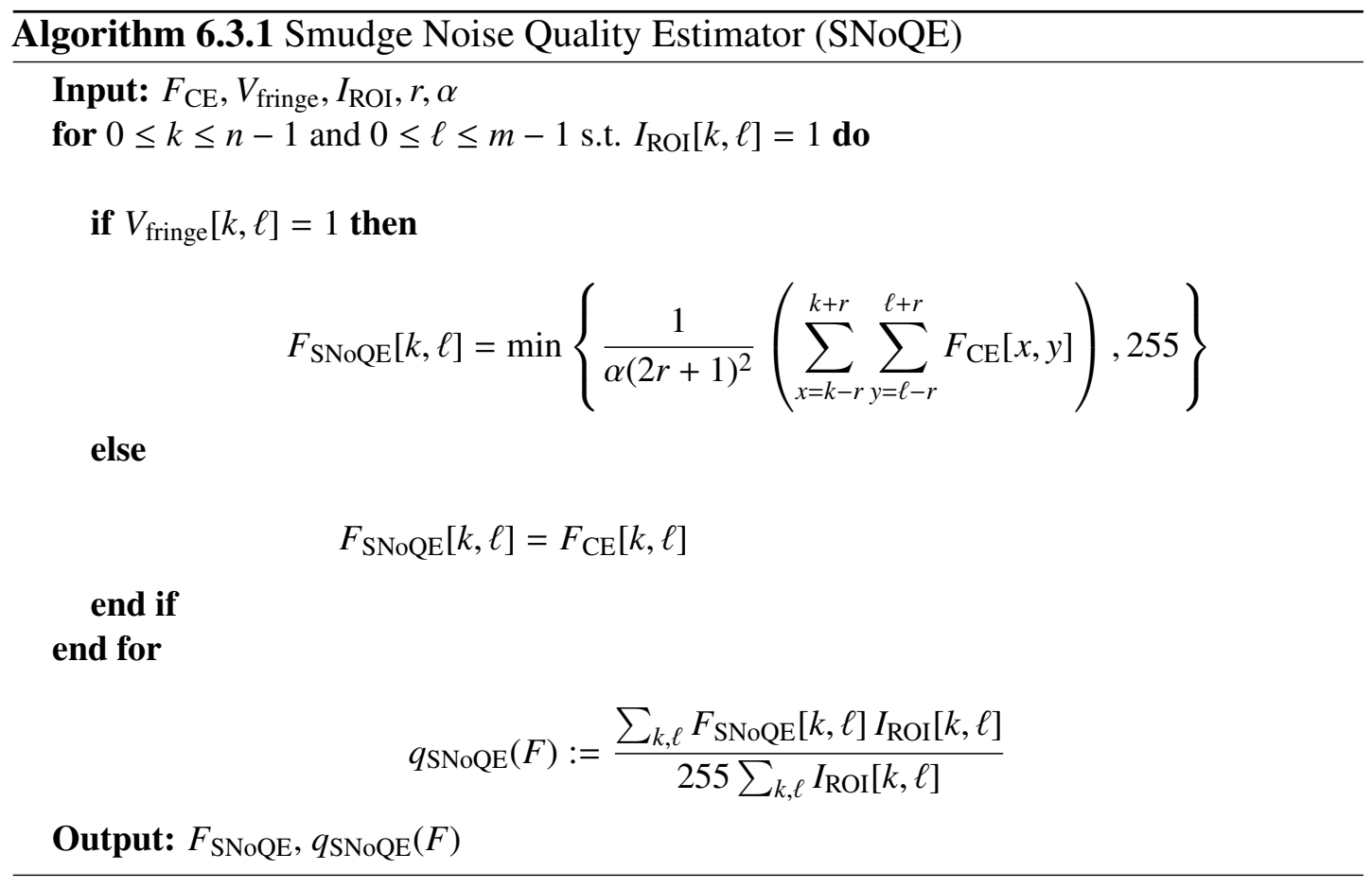

of the first type do not contribute to the fringe pattern, i.e. $V_{\text {fringe }}[k, \ell]=0$, and we set $F_{\mathrm{CE}}[k, \ell]$ as their smudge value. For smudge-like pixels, as explained above, these values are close to zero, indicating low quality. Pixels of the second type contribute to the fringe pattern, i.e. $V_{\text {fringe }}[k, \ell]=1$. We assign such pixels good quality (a value close to 255) if they contain within a block of integer radius $r$ (chosen such that $2 r+1$ pixels slightly exceed average ridge width) at least as many valley pixels ( $\alpha$ times block size) as expected for a good quality fingerprint pixel located in the "worst" position, namely in the center of a ridge, cf. Figure 6.4, that may even be a minutia center. For detailed pseudo-code see Algorithm 6.3.1.

Dryness noise estimation is performed in the same way, with the following obvious difference. For the dryness noise quality estimator (DNoQE) the routine of Algorithm 6.3.1 is applied to the grey-scale inverted $255-F_{\mathrm{CE}}[k, \ell]$. If we simultaneously penalize for smudge and dryness and assign to each pixel the minimum of the calculated smudge value and of its dryness counterpart this leads to the smudge and dryness noise quality estimator (SaDNoQE) given as:

$$
F_{\mathrm{SaDNoQE}}[k, \ell]:=\min \left\{F_{\mathrm{SNoQE}}[k, \ell], F_{\mathrm{DNoQE}}[k, \ell]\right\} .
$$

For almost all of the databases investigated in Chapter 6.5, among the here introduced quality features SNoQE yields by far the best results. Leading to the conjecture that with respect to the scanning devices and comparison subsystems used, spurious and missed minutiae detection are much more linked to smudge noise and much less to 

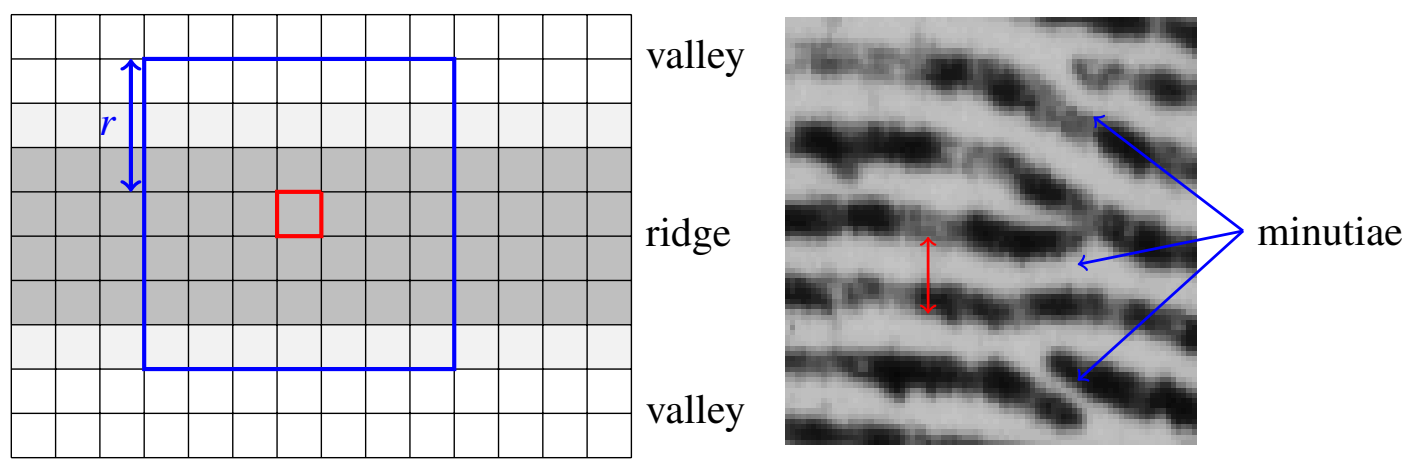

Figure 6.4: On the left: Schematic illustration for SNoQE parameters in case of $R_{\text {Med }}=10$ and $R_{0.95}=12$, yielding $r=3$ and $\alpha=\frac{14}{49}$ (the red square represents $[k, \ell]$, the blue square represents the local neighbourhood of $[k, \ell])$. On the right: Example of minutiae in a fingerprint (ridge endings and bifurcations), as well as, a depiction of the inter-ridge distance, the pixel distance of a valley and a ridge (red double arrow).

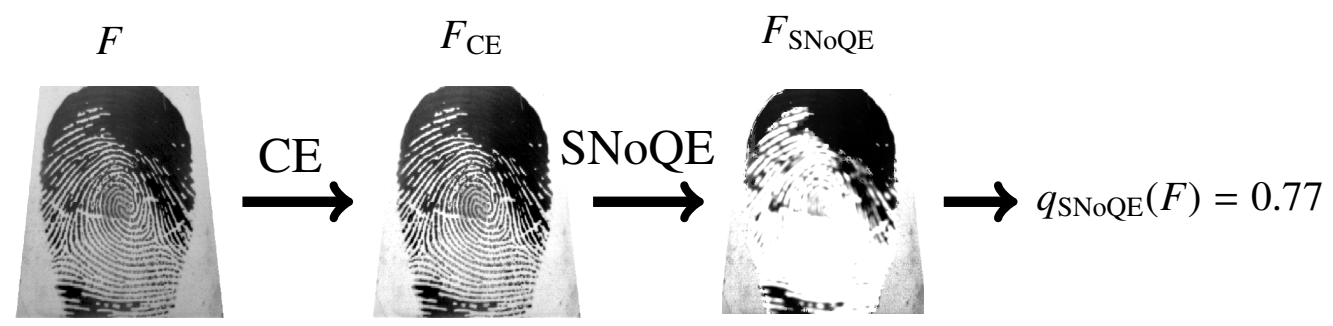

Figure 6.5: SNoQE for the example in Figure 6.3, with $r=3$ and $\alpha=3.0093$.

dryness noise.

\subsubsection{Parameter choices for $r$ and $\alpha$}

Recall that the inter-ridge distance (IRD) is a local feature of a fingerprint, see the right of Figure 6.4, giving the local distance between two neighbouring ridge lines. Depending on scanner and fingerprint, this feature varies over a small but considerable interval. For each fingerprint its average IRD are computed, and for a given database, denote by $R_{\text {med }}$ the median and by $R_{0.95}$ the $95 \%$ quantile of the distribution of averaged IRDs. These numbers form the basis for our choices of $r$ and $\alpha$, which are chosen such that for a good quality fingerprint every $(2 r+1) \times(2 r+1)$ block contains at least a fraction of $\alpha$ valley pixels $[k, \ell]$, i.e. pixels with $F_{\mathrm{CE}}[k, \ell]=255$. Setting

$$
r:=\left\lceil\frac{R_{95}}{4}\right\rceil
$$

and under the simplifying assumption that locally the fingerprint ridges are straight lines, as in the left of Figure 6.4 , we ensure that in a $(2 r+1) \times(2 r+1)$ box of a good quality 
fingerprint at least $\alpha(2 r+1)^{2}$ pixels feature $F_{\mathrm{CE}}[k, \ell]=255$. On the left of Figure6.4 we depict the "(second) worst" case, namely that the center of the box is the center of a ridge line where $F_{\mathrm{CE}}$ assumes the value zero. Here we have the equivalent of $2(2 r+1)$ white pixels (including the $2(2 r+1)$ half white pixels accounting for $2 r+1$ white pixels). In the "worst case" the box's center is also a minutia center such that most of the upper valley part on the left of Figure 6.4 is no longer white and we can only ensure the equivalent of $2 r+1$ white pixels, i.e. $\alpha=(2 r+1)^{-1}$ in this case. It turns out, due to overestimation of $r$ by the 0.95 quantile in (6.3.1), that this number is too small and the following is a good and robust choice

$$
\alpha:=\frac{1}{2 r+1}\left(1+2\left\lceil\frac{R_{95}}{4}\right\rceil-\frac{R_{\mathrm{Med}}}{2}\right) \geq \frac{1}{2 r+1} .
$$

A detailed analysis of the parameters $r$ and $\alpha$ in Appendix D shows that they are mildly robust against misspecification, see Figure D.4 and D.5.

\subsection{Robust Biometric Quality Validation Scheme (RBQ VS)}

Validating the performance of a quality estimator is a non-trivial task, since there is no ground truth for the quality of a fingerprint image. In the following a procedure is proposed that assesses a biometric quality estimator considering its ability to lower errors of a given comparison subsystem, making the comparison data our ground truth. As discussed in Chapter 6.1 there are many approaches to validate whether the assignment of good quality to two biometric queries with respect to a quality estimator relates to lowered probability of comparison errors. While feature vectors, like, for example, ERC curves, serve well for descriptive purposes, for our task at hand, we require a single scalar quantification of its performance. However, to employ a quality estimator a quality threshold needs to be trained on a training set beforehand with regard to lowering, for example, the EER of a comparison subsystem.

To mimic this use-case and validate, for a candidate pair of a quality estimator and a comparison subsystem, we divide a given database into a training and a test set, then train a quality threshold on the training set and impose this trained threshold on the test set. This was done in Lee et al. [LCCK08] for a small training set as one validation among others. Often, however, due to high variation, the outcome depends on the specific division of the database into training and test set, and indeed, this happens for our use-case, see Chapter 6.5. In consequence, the procedure is robustified here by $K$ runs ( $K$ sufficiently large) of repeated random sub-sampling. 


\subsection{Robust Biometric Quality Validation Scheme (RBQ VS)}

Here is the formal definition of the RBQ VS: Assuming that biometric samples (fingerprints in our case) have been taken from varying individuals, and all measurement have been collected in a database $Y$ with $N_{\text {indv }} \in \mathbb{N}$ different individuals (fingers), where each individual (finger) contributes $N_{\text {repl }} \in \mathbb{N}$ replicate samples (prints), view a comparison subsystem as a map $m: Y \times Y \rightarrow[0,1]$ assigning each pair a comparison score indicating the degree of similarity between the two measurements. For a given threshold $t_{\text {match }}$ two measurements $i$ and $j$ match whenever $m(i, j)>t_{\text {match }}$.

For assessment, we define the well known false matches (FM) and the false non-matches (FNM) with respect to $t_{\text {match }}$ as well as the ground truth of genuine attempts (GA) and imposter attempts (IA), respectively, see Maltoni et al. [MMJP09], as

$F M\left(t_{\text {match }}\right)=\#\left\{m(i, j)>t_{\text {match }}\right.$ and $i$ and $j$ are not from the same individual $\}$,

$F N M\left(t_{\text {match }}\right)=\#\left\{m(i, j) \leq t_{\text {match }}\right.$ and $i$ and $j$ are from the same individual $\}$,

$G A=\#\{(i, j) \in Y \times Y$ and $i$ and $j$ are from the same individual $\}$,

$I A=\#\{(i, j) \in Y \times Y$ and $i$ and $j$ are not from the same individual $\}$.

Since, in general, $I A$ is not equal to $G A$, we consider the false match rate and the false non-match rate,

$$
\begin{aligned}
F M R\left(t_{\text {match }}\right) & :=\frac{F M\left(t_{\text {match }}\right)}{I A}, \\
F N M R\left(t_{\text {match }}\right) & :=\frac{F N M\left(t_{\text {match }}\right)}{G A} .
\end{aligned}
$$

Then

$$
\left\{\left(F M R\left(t_{\text {match }}\right), F N M R\left(t_{\text {match }}\right)\right): t_{\text {match }} \in[0,1]\right\}
$$

defines the receiver operating characteristic (ROC) curve. In practice one aims at meeting a pre-specified FMR or FNMR, or at maximizing the area under the ROC curve (AUC), or at minimizing the equal error rate (EER), e.g. [MMWJ02], given by

$$
t_{E E R}:=\underset{t_{\text {match }} \in[0,1]}{\arg \min }\left|F M R\left(t_{\text {match }}\right)-F N M R\left(t_{\text {match }}\right)\right| .
$$

If $q: Y \rightarrow[0,1]$ is now a quality estimator, here is the protocol of the RBQ VS meeting the objective of minimizing the EER. Protocols for meeting other objectives are then straightforward.

(1) Input: Number of runs $K \in \mathbb{N}$, size $X$ of the training set $0<X<N_{\text {indv }}$ and the number of quality bins $L \in \mathbb{N}$.

(2) Do for $k=1,2, \ldots, K$ :

(2.1) Randomly select $X$ individuals. All replicate measurements from the se- 
lected individuals will constitute the training set $Y_{\text {train }}$, the replicate measurements from all other individuals comprise the test set $Y_{\text {test }}$.

(2.2) Do for $j=0, \frac{1}{L}, \frac{2}{L}, \ldots, 1$ :

(2.2.1) Define the quality enhanced training set $Y_{\text {train }}^{(j)}$ of all replicate measurements with quality higher than $j$ :

$$
Y_{\text {train }}^{(j)}:=\left\{f \in Y_{\text {train }}: q(f) \geq j\right\}
$$

(2.2.2) If for $Y_{\text {train }}^{(j)}$ either $G A=0$ or $I A=0$, set $E E R_{j}:=1$.

(2.2.3) Else, compute the EER of $Y_{\text {train }}^{(j)}$ and denote it by $E E R_{j}$.

(2.3) Compute

$$
j^{*}:=\arg \min _{j}\left(E E R_{j}\right)
$$

(2.4) Define the quality enhanced test set $Y_{\text {test }}^{*}$ of all replicate measurements with quality higher than $j^{*}$ :

$$
Y_{\text {test }}^{*}:=\left\{f \in Y_{\text {test }}: q(f) \geq j^{*}\right\} .
$$

(2.5) If the $I A$ and $G A$ of $Y_{\text {test }}^{*}$ is not 0 the run is valid and the ROC curve and EER for $Y_{\text {test }}^{*}$ are computed.

(3) Average the results of each valid run to obtain an averaged EER and ROC curve.

Upon completion, we can obtain and compare the resulting empirical distributions of the EER, or the ROC, or the AUC as well as their mean or their median, et cetera.

\subsection{Comparison Study}

For comparison we use the RBQ VS based on mean EERs, allowing for scalar ranking. Further, we provide ERC curves linking FNMRs to fractions of genuine attempts excluded.

We conduct our study on the popular and open source FVC databases and on the NIST SD4 database of rolled imprints. The FVC databases have been obtained employing various sensors: low (2000 DB1) and high cost optical sensors (2000 DB2, 2002 DB1/DB2 and 2004 DB1/DB2), low (2000 DB3) and high cost capacitive sensors (2002 DB3) and a thermal sensor (2004 DB3), cf. Maio et al. [MMWJ02] and Tab. 6.3. Each of the nine FVC databases contains 880 fingerprints with $N_{\text {indv }}=110$ individual fingers, each with $N_{\text {repl }}=8$ replicates. The NIST SD4 contains 4000 fingerprints with 
Table 6.3

Sensors used by the FVC databases

\begin{tabular}{c|c|c}
\hline Database & Sensor & Resolution \\
\hline FVC2000 DB1 & "Secure Desktop Scanner" by KeyTronic (Low-cost optical) & $500 \mathrm{dpi}$ \\
FVC2000 DB2 & "TouchChip" by ST Microelectronics (Low-cost capacitive) & $500 \mathrm{dpi}$ \\
FVC2000 DB3 & "DF-90" by Identicator Technology (optical) & $500 \mathrm{dpi}$ \\
FVC2002 DB1 & "Touch View II" by Identix (optical) & $500 \mathrm{dpi}$ \\
FVC2002 DB2 & "FX2000" by Biometrika (optical) & $569 \mathrm{dpi}$ \\
FVC2002 DB3 & "100 SC" by Precise Biometrics (capacitive) & $500 \mathrm{dpi}$ \\
FVC2004 DB1 & "V300" by CrossMatch (optical) & $500 \mathrm{dpi}$ \\
FVC2004 DB2 & "U.are.U 4000" by Digital Persona (optical) & $500 \mathrm{dpi}$ \\
FVC2004 DB3 & "FingerChip FCD4B14CB" by Atmel (thermal) & $512 \mathrm{dpi}$ \\
\hline
\end{tabular}

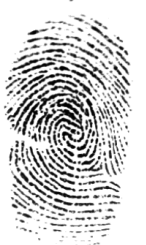

(a)

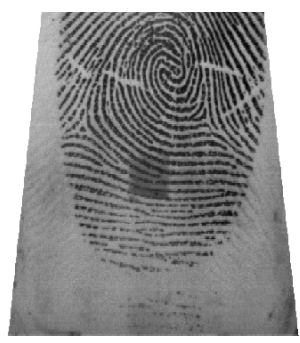

(b)

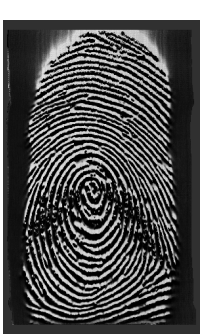

(c)

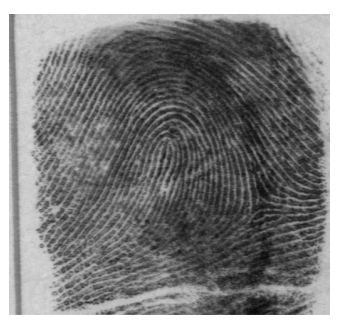

(d)

Figure 6.6: Example fingerprints from the FVC and the NIST SD4: optical (a), thermal (b), capacitive (c) and rolled (d).

$N_{\text {indv }}=2000$ individual fingers and $N_{\text {repl }}=2$ replicate fingerprints each. Typical images of the FVC databases and NIST SD4 are displayed in Figure 6.6.

For quality estimators SNoQE, as well as NFIQ 2.0 are used. Since it turns out that its first version NFIQ performs differently and sometimes outperforms NFIQ 2.0, it is also included. Moreover, a simple combination of SNoQE with NFIQ 2.0 is studied, denoted by SNoQE $\wedge$ NFIQ2. For the RBQ VS this combination is obtained by, setting

$$
\tilde{Y}_{\text {test }}^{*}:=\left\{F \in Y_{\text {test }}: q_{\mathrm{SNoQE}}(F) \geq j_{\mathrm{SNoQE}}^{*} \text { and } q_{\mathrm{NFIQ} 2}(F) \geq j_{\mathrm{NFIQ} 2}^{*}\right\}
$$

enforcing both, independently trained quality thresholds at once. For the ERC curves considered below, this combination is obtained by first performing histogram spreading for $q_{\mathrm{SNoQE}}$ and $q_{\mathrm{NFIQ} 2}$, to exhaust the full interval [0,1], and then setting

$$
q_{\mathrm{SNoQE} \wedge \mathrm{NIFQ} 2}(F):=\min \left\{q_{\mathrm{SNoQE}}(F), q_{\mathrm{NFIQ} 2}(F)\right\} .
$$

Recall from Chapter 6.2 that the G3PD parameters have been specifically trained in [TG16] for ROI extraction on the nine FVC databases; as the NIST SD4 is visually similar to FVC 2000 DB3, the same parameters are used, cf. Tab 6.2, which turn out to 
Table 6.4

IRD statistics defining parameters $r$ and $\alpha$ in (6.3.1) and (6.3.2) using histograms of [Got12] for the FVC databases and heuristic estimates for NIST SD4, cf. Figure D.3 in Appendix D.

\begin{tabular}{c|c|c|c|c}
\hline Database & $R_{95}$ & $R_{\mathrm{Med}}$ & $\alpha$ & $r$ \\
\hline FVC2000 DB1 & 10.6226 & 9.2353 & 0.3403 & 3 \\
FVC2000 DB2 & 10.6332 & 9.1034 & 0.3496 & 3 \\
FVC2000 DB3 & 12.0301 & 10.5852 & 0.4119 & 4 \\
FVC2002 DB1 & 9.9851 & 8.8957 & 0.2868 & 3 \\
FVC2002 DB2 & 12.0931 & 10.3204 & 0.4266 & 4 \\
FVC2002 DB3 & 10.5428 & 8.9698 & 0.3593 & 3 \\
FVC2004 DB1 & 10.7047 & 9.0918 & 0.3506 & 3 \\
FVC2004 DB2 & 11.0235 & 9.3484 & 0.3323 & 3 \\
FVC2004 DB3 & 10.2965 & 9.1005 & 0.3500 & 3 \\
NIST SD4 & 17 & 14 & 0.3636 & 5 \\
\hline
\end{tabular}

be quite satisfactory. Recall further from Chapter 6.3 that SNoQE's parameters $r$ and $\alpha$ are based on the median of the distribution of averaged IRDs of the database $R_{\text {Med }}$ and the corresponding 0.95 quantile $R_{95}$. Again for the nine FVC databases, the latter quantities have been determined (cf. [Got12]) and for the NIST SD4, they have been heuristically estimated, cf. Table 6.4 and Figure D.3 in Appendix D.

As comparison subsystems (CS) the publicly available bozorth3 of the NIST [NFI15] and, additionally, the commercial "FingerCode 3" of the Matching SDK by Dermalog $\mathrm{GmBH}$, in the following denoted by commercial, are used.

\subsubsection{RBQ VS Results}

For the RBQ VS the number of runs is set to $K=100$, in order to guarantee robustness, the portion of the training $X$ is chosen as around $1 / 4$ of $N_{\text {indv }}$, but not too small, here we choose $X=40$ for the FVC databases and $X=500$ for the NIST SD4. By construction, NFIQ has only $L=5$ quality bins, NFIQ 2.0 has $L=100$ and for SNoQE the same number $L=100$ is used. These bins are embedded in $[0,1]$ with 1 for highest quality. Notably, for small databases such as the FVC, employing too fine binning, risks to obtain runs, for which, after a quality threshold has been trained, the set $Y_{\text {test }}^{*}$ of (6.4.2) has no remaining genuine attempts, cf. comment below Table 6.5.

In Table 6.5 the means of the resulting EER distributions over the quality enhanced test sets (from Chapter 6.4) are displayed for each database, CS and quality estimator, respectively. As baseline of the RBQ VS the mean EER of all test sets without quality assessment is shown in the fourth column. Out of 20 cases considered, SNoQE performs best in 6, NFIQ 2.0 in 8 and, surprisingly, NFIQ in the remaining 6 cases. Upon closer 


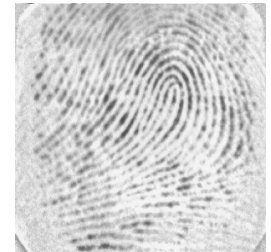

(a)

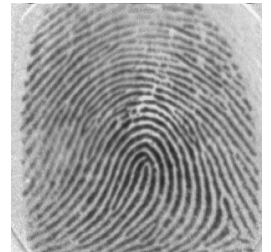

(b)

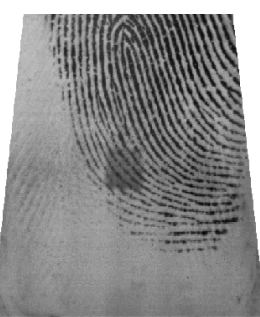

(c)

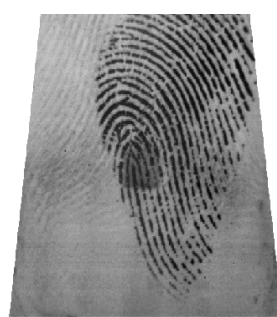

(d)

Figure 6.7: Replicates 2 (a) and 7 (b) of finger 52 from FVC 2000 DB1. Both prints have high NFIQ 2.0 score (75 and 59, respectively) but a low comparison score resulting in a false non-match. Replicates 1 (c) and 2 (d) of finger 106 from DB2. Both prints have high SNoQE quality (0.9767 and 0.9637 , respectively), while only 3 (or 5 , including the miniature island near the core) minutiae overlap. This leads to a reject by both CSs.

Table 6.5

Mean EERs for the quality enhanced test sets. No quality thresholding gives the baseline. Lowest scoring single quality estimator in bold. SNoQE $\wedge$ NFIQ2 scores are always lower and marked italic.

\begin{tabular}{c|c|c|c|c|c|c||c}
\hline Database & Comp. Subsystem & Sensor type & Baseline & NFIQ & NFIQ 2.0 & SNoQE & SNoQE $\wedge$ NFIQ2 \\
\hline FVC2000 DB1 & bozorth3 & optical & 0.0490 & 0.0139 & 0.0248 & $\mathbf{0 . 0 0 4 3}$ & 0.0012 \\
FVC2000 DB1 & commercial & optical & 0.0138 & 0.0036 & $\mathbf{0 . 0 0 2 1}$ & 0.0033 & 0.0008 \\
FVC2000 DB2 & bozorth3 & capacitive & 0.0482 & 0.0144 & 0.0056 & $\mathbf{0 . 0 0 2 2}$ & 0.0001 \\
FVC2000 DB2 & commercial & capacitive & 0.0100 & $\mathbf{0 . 0 0 0 1}$ & 0.0008 & 0.0012 & 0.0003 \\
FVC2000 DB3 & bozorth3 & optical & 0.1324 & 0.0118 & $\mathbf{0 . 0 0 3 5}$ & 0.0614 & 0.0001 \\
FVC2000 DB3 & commercial & optical & 0.0410 & 0.0089 & $\mathbf{0 . 0 0 3 7}$ & 0.0146 & 0.0037 \\
\hline FVC2002 DB1 & bozorth3 & optical & 0.0333 & $\mathbf{0 . 0 1 1 7}$ & 0.0125 & 0.0345 & 0.0030 \\
FVC2002 DB1 & commercial & optical & 0.0129 & 0.0053 & $\mathbf{0 . 0 0 5 2}$ & 0.0137 & 0.0036 \\
FVC2002 DB2 & bozorth3 & optical & 0.0280 & 0.0059 & 0.0037 & $\mathbf{0 . 0 0 3 2}$ & 0 \\
FVC2002 DB2 & commercial & optical & 0.0067 & 0.0018 & $\mathbf{0 . 0 0 0 8}$ & 0.0017 & 0 \\
FVC2002 DB3 & bozorth3 & capacitive & 0.3236 & $\mathbf{0 . 0 2 3 2}$ & 0.0815 & 0.0672 & 0 \\
FVC2002 DB3 & commercial & capacitive & 0.0271 & 0.0066 & $\mathbf{0 . 0 0 6 3}$ & 0.0074 & 0.0037 \\
\hline FVC2004 DB1 & bozorth3 & optical & 0.1118 & 0.079 & $\mathbf{0 . 0 2 6 5}$ & 0.0527 & 0.0020 \\
FVC2004 DB1 & commercial & optical & 0.0569 & 0.0500 & 0.0219 & $\mathbf{0 . 0 0 5 4}$ & 0.0002 \\
FVC2004 DB2 & bozorth3 & optical & 0.1125 & $\mathbf{0 . 0 0 1 2}$ & 0.0182 & 0.0540 & 0.0001 \\
FVC2004 DB2 & commercial & optical & 0.0427 & $\mathbf{0 . 0 0 0 4}$ & 0.0042 & 0.0155 & 0.0001 \\
FVC2004 DB3 & bozorth3 & thermal & 0.0743 & 0.0347 & 0.0075 & $\mathbf{0 . 0 0 4 3}$ & 0.0032 \\
FVC2004 DB3 & commercial & thermal & 0.0266 & 0.0115 & $\mathbf{0 . 0 0 0 7}$ & 0.0009 & 0.0001 \\
\hline NIST SD4 & bozorth3 & rolled & 0.0457 & $\mathbf{0 . 0 0 0 2}$ & 0.0019 & 0.0011 & $10^{-6}$ \\
NIST SD4 & commercial & rolled & 0.0265 & 0.0005 & 0.0013 & $\mathbf{0 . 0 0 0 4}$ & $3 \times 10^{-6}$ \\
\hline
\end{tabular}

Comment: Whenever on a database a run had no remaining genuine attempts for one of the three single quality estimators, this run was disregarded for all three, including baseline (13 runs in 2000 DB3 with bozorth3 and 6 runs in 2000 DB3 with commercial, 2 in 2002 DB3 with bozorth3, 11 in 2004 DB1 with bozorth3, 14 in 2004 DB2 with bozorth3 and 6 in 2004 DB3 with commercial). For the combination (last column) we excluded only runs producing no remaining genuine attempts for the combination (5 in 2000 DB1 with commercial, 49 with bozorth and 44 with commercial in 2000 DB3, 32 in 2002 DB2 with bozorth3, 92 with bozorth and 34 with commercial in 2002 DB3, 23 in 2004 DB1 with bozorth3, 10 in 2004 DB1 with commercial, 69 in 2004 DB2 with bozorth3, 83 in 2004 DB2 with commercial and 3 in 2004 DB3 with bozorth3).

inspection, in 2 cases all three quality estimators perform similarly. Furthermore, in 2 cases SNoQE and NFIQ 2.0 outperform NFIQ, in 2 cases NFIQ and NFIQ 2.0 outperform SNoQE and in one case SNoQE and NFIQ outperform NFIQ 2.0. For each one of the three, there are also cases where they strongly outperform the other 
two. Since NFIQ 2.0 has been trained on optical sensors, there is a tendency that it is outperformed by SNoQE on thermal and capacitive sensors. The proposed combination SNoQE $\wedge$ NFIQ2 from (6.5.1), however, clearly outperforms all competitors. Thus by construction of SNoQE $\wedge$ NFIQ2, employing thresholds on the NFIQ 2.0 and the SNoQE quality scores lowers comparison errors over all databases. In view of usability, we note that by construction, $\mathrm{SNoQE} \wedge$ NFIQ2 tends to reject more prints from matching than the other estimators as can be seen in the comment of Table 6.5, and in Table 6.6 for the NIST SD4.

\subsubsection{ERC Curves}

Following Grother and Tabassi [GT07] ERC curves are calculated by disregarding genuine attempts from matching if one of the two samples does not exceed the quality threshold. This is one of many validation schemes discussed in [GT07] and it conforms with the rationale underlying the RBQ VS. For each database we compute the comparison threshold at 0.1 FNMR and plot the FNMR against the fraction of genuine attempts excluded from comparison as dictated by the quality estimator. For the combined quality estimator SNoQE $\wedge$ NFIQ2, cf. (6.5.2).

In Figure 6.8 and 6.9 we present the ERC curves for NFIQ, SNoQE, NFIQ 2.0 and $\mathrm{SNoQE} \wedge$ NFIQ2, the combination of the latter two, using bozorth3 and commercial, respectively. The results are very mixed, endorsing the hypothesis that SNoQE and NFIQ 2.0 measure different quality aspects. For example, on FVC 2002 DB3 (capacitive sensor) SNoQE consistently outperforms NFIQ 2.0 for bozorth3, and vice versa NFIQ 2.0 consistently outperforms SNoQE on FVC 2002 DB1 (optical sensor). On FVC 2000 DB1 (optical sensor) with bozorth3, for high exclusion NFIQ 2.0 consistently outperforms SNoQE, while for low exclusion, SNoQE consistently outperforms NFIQ 2.0. This relationship is reversed for FVC 2000 DB3 (thermal sensor) with bozorth3. For the other databases (most are optical), ERC curves of SNoQE and NFIQ 2.0 can be very close, often winding around each other, giving no clear "winner", their combination SNoQE $\wedge$ NFIQ2, however, often outperforms. Notably, for some databases and CSs, ERC points of NFIQ are below NFIQ 2.0, sometimes also below SNoQE, and occasionally even below their combination. ERC curves of DNoQE are shown in Figure D.2 in Appendix D, exemplifying that for the databases of concern, dryness noise, as measured by DNoQE, is of little interest for quality assessment.

For the NIST SD4 the result in (d) and (e) of Figure 6.9 is surprising: The old NFIQ outperforms the new NFIQ 2.0 for both CSs. Since the underlying rolled fingers have been carefully taken following a forensic protocol, this database contains little smudge and hence both NFIQs outperform SNoQE. Still, all three prove to relate quality to 


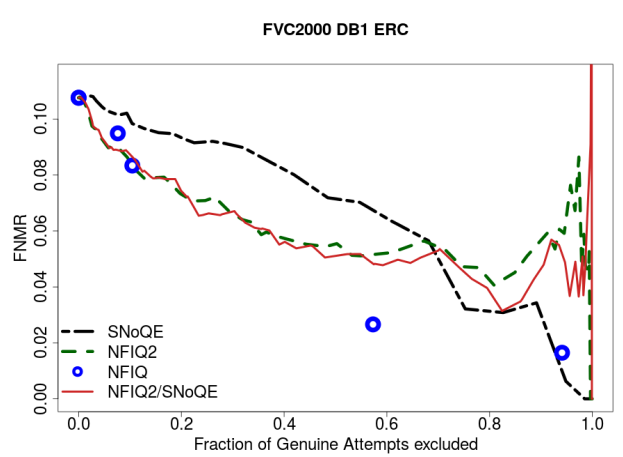

(a)

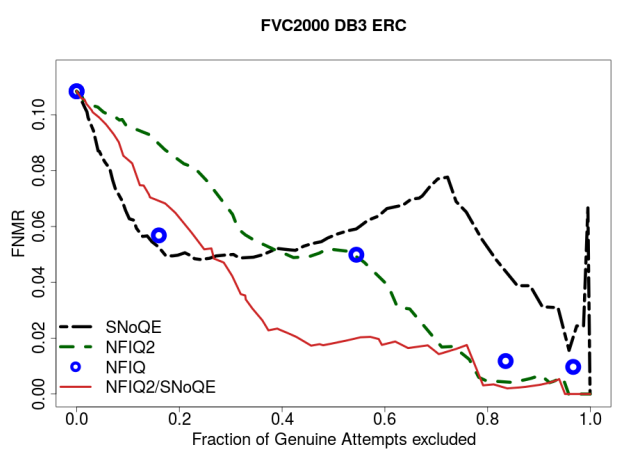

(c)

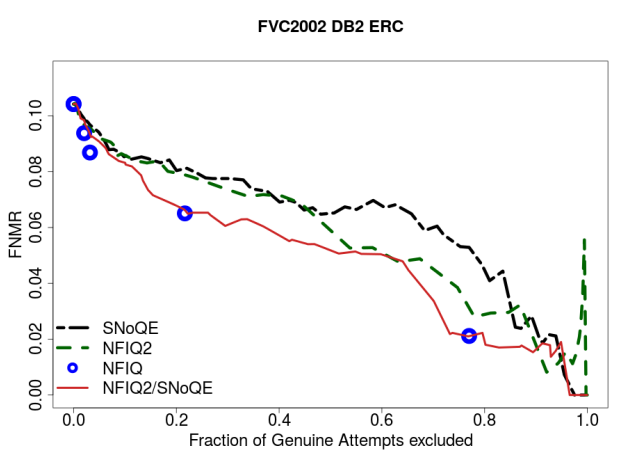

(e)

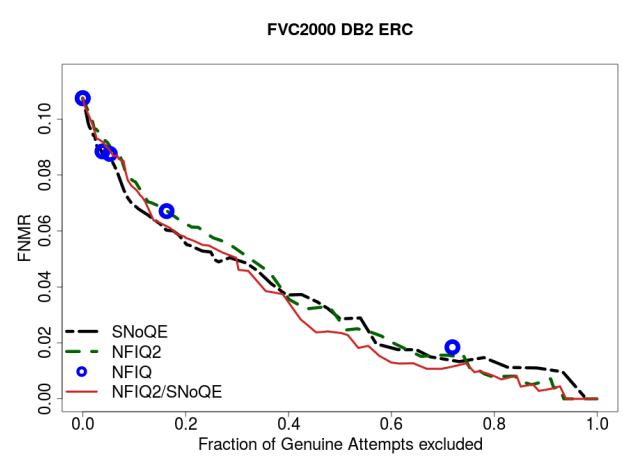

(b)

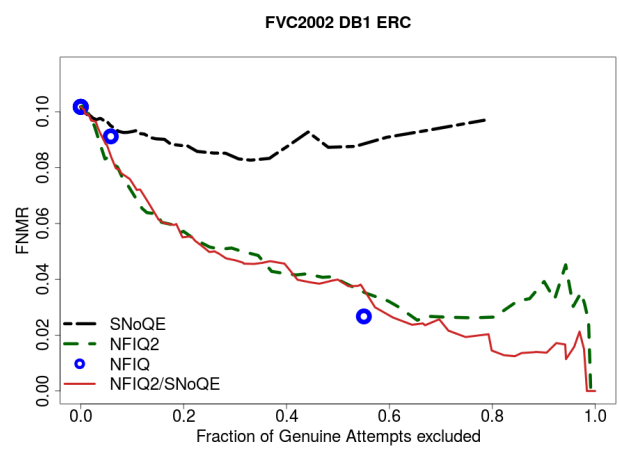

(d)

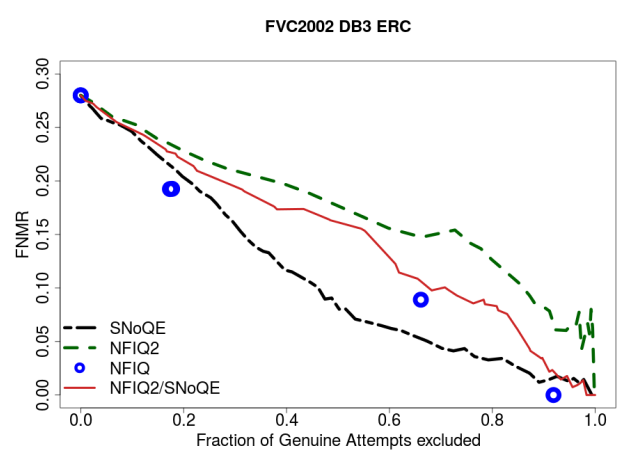

(f)

Figure 6.8: ERC curves for the FVC databases 2000 and 2002 under bozorth3. 


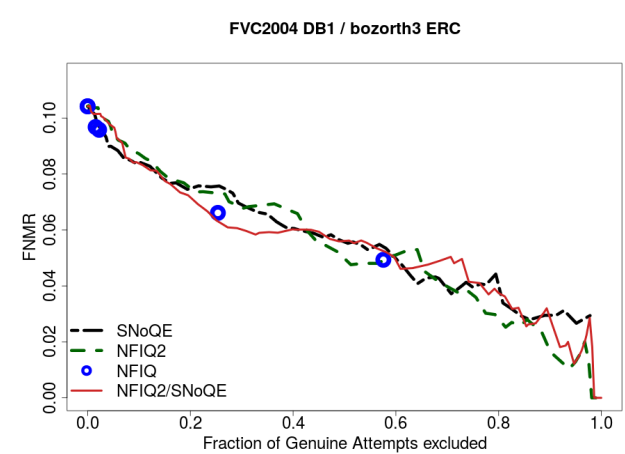

(a)

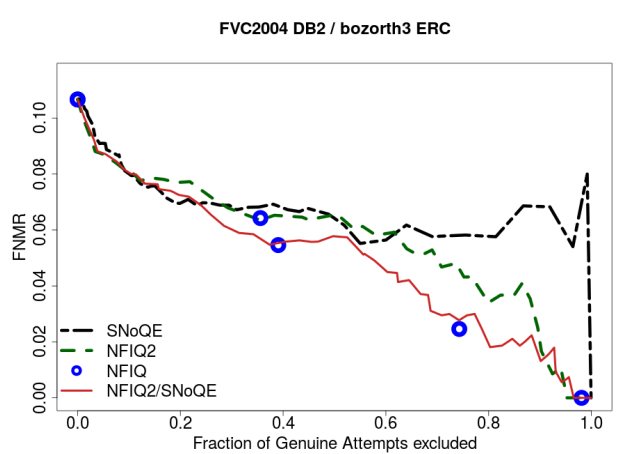

(b)

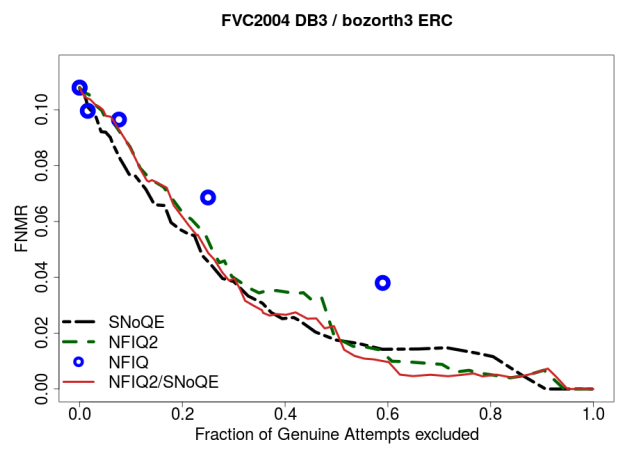

(c)

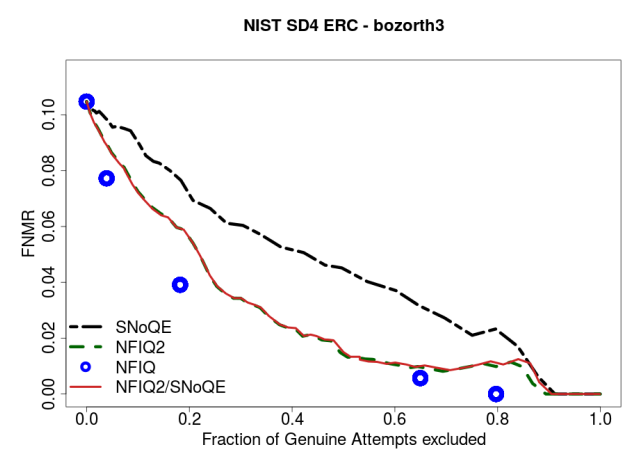

(d)

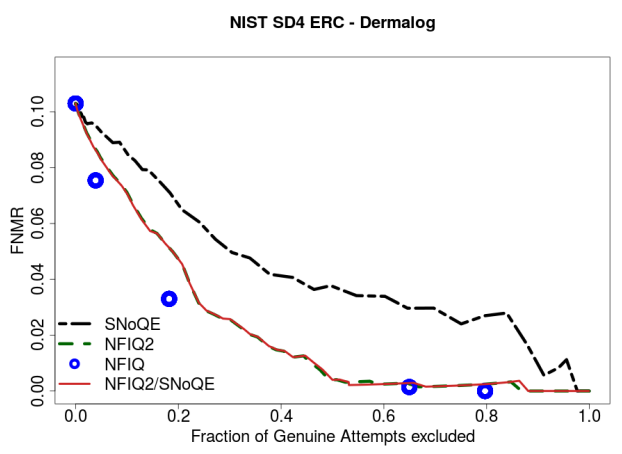

(e)

Figure 6.9: ERC curves for the FVC database 2004 under bozorth3 ((a) to (c)) and ERC curves for the NIST SD4 under bozorth3 (d) and commercial (e). 
Table 6.6

Average number of prints rejected by employment of a quality threshold within the

RBQ VS

\begin{tabular}{c||c|c}
\hline Database & NIST SD4 & NIST SD4 \\
\hline Comp. Subsystem & bozorth3 & commercial \\
\hline NFIQ & 1894 & 1554 \\
NFIQ 2.0 & 2184 & 1360 \\
SNoQE & 2403 & 2332 \\
SNoQE $\wedge$ NFIQ2 & 2713 & 2492 \\
\hline
\end{tabular}

false non-matches. While the RBQ VS shows that a combination of NFIQ 2.0 and SNoQE outperforms each single quality estimator, this effect is almost invisible in (d) and (e) of Figure 6.9 suggesting the study of more advanced combinations. Note that Figure D.1 in Appendix D stresses that, also for NIST SD4, NFIQ 2.0 and SNoQE measure different quality aspects.

\subsubsection{Delineation of RBQ VS from ERC Diagnostics}

Recall that a quality estimator is deemed favorable under ERC diagnostics if it features a quickly decaying ERC curve. In contrast, it is deemed favorable under RBQ VS if it results in low EER, where there is no penalty on the number of prints rejected as long as genuine attempts remain possible. In consequence, ERC diagnostics and RBQ ranking validate different aspects of a quality estimator. However, the following commonality of tail behavior of ERC curves with RBQ VS can be observed, in Figure 6.8 and 6.9. and Table 6.5: If for a quality estimator for high exclusion rate, FNMRs are considerably above zero, it cannot perform well under RBQ VS. Notably, for small databases as the FVC databases "bad tail behaviors" observable in FVC 2000 DB1 with bozorth3 for NFIQ 2.0 and FVC 2004 DB2 with bozorth3 for SNoQE can result from "outlier" prints that have a high quality score but cause a non-match error, see Figure 6.7.

At this point it is important to remark that RBQ VS is a conservative validation scheme, as it imposes effectively no penalty on the number of prints excluded from comparison. Hence, it models a high-security use-case in which the number of rejected prints can be very high (cf. comment to Table 6.5 and Table 6.6) resulting in a high number of expected retries. However, if usability is of concern (i.e. low-security), the RBQ VS can be relaxed if a penalty is introduced in Step (2.2.2/2.2.3), see Chapter 6.4.

An advantage of the RBQ VS lies in robust quantification of its results for the use-case modelled. Recall that, in order to guarantee robustness, we used repeated random subsampling. This allows, in contrast to ERC diagnostics, to state assertions with 
Table 6.7

Number of runs in which the resulting EER of NFIQ 2.0 or SNoQE was lower, respectively, under the bozorth3 comp. subsystem.

\begin{tabular}{c||c|c|c}
\hline Database & NFIQ 2.0 & Equal EER & SNoQE \\
\hline FVC2000 DB1 & 0 & 37 & $\mathbf{6 3}$ \\
FVC2000 DB2 & 35 & 5 & 60 \\
FVC2000 DB3 & 75 & 5 & 14 \\
FVC2002 DB1 & $\mathbf{9 9}$ & 0 & 1 \\
FVC2002 DB2 & 50 & 19 & 31 \\
FVC2002 DB3 & 41 & 2 & 55 \\
FVC2004 DB1 & 76 & 0 & 8 \\
FVC2004 DB2 & 63 & 7 & 19 \\
FVC2004 DB3 & 21 & 11 & 68 \\
NIST SD4 & 28 & 1 & 71 \\
\hline
\end{tabular}

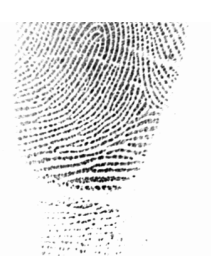

(a)

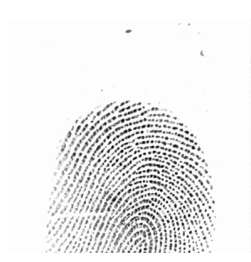

(b)

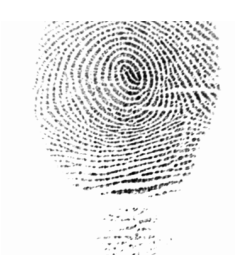

(c)

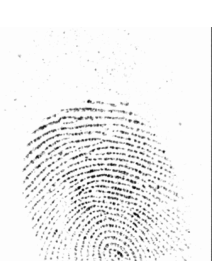

(d)

Figure 6.10: Replicates 3 (a), 4 (b) of finger 89 and replicates 5 (c), 7 (d) of finger 100 of FVC 2002 DB1. While having a high SNoQE score $(0.9985,1,0.9986$ and 0.9999, respectively) all feature a low comparison scores, due to partial overlap.

Table 6.8

Computational runtimes

\begin{tabular}{c|c|c}
\hline Database & NFIQ 2.0 & SNoQE \\
\hline FVC2000 DB1 & $77454 \mathrm{~s}$ & $16081 \mathrm{~s}$ \\
FVC2000 DB2 & $77492 \mathrm{~s}$ & $16658 \mathrm{~s}$ \\
FVC2000 DB3 & $175563 \mathrm{~s}$ & $33732 \mathrm{~s}$ \\
FVC2002 DB1 & $98014 \mathrm{~s}$ & $23066 \mathrm{~s}$ \\
FVC2002 DB2 & $147797 \mathrm{~s}$ & $27801 \mathrm{~s}$ \\
FVC2002 DB3 & $75594 \mathrm{~s}$ & $15233 \mathrm{~s}$ \\
FVC2004 DB1 & $83973 \mathrm{~s}$ & $44814 \mathrm{~s}$ \\
FVC2004 DB2 & $104160 \mathrm{~s}$ & $20233 \mathrm{~s}$ \\
FVC2004 DB3 & $127322 \mathrm{~s}$ & $23325 \mathrm{~s}$ \\
NIST SD4 & $786655 \mathrm{~s}$ & $27683 \mathrm{~s}$ \\
\hline
\end{tabular}


statistical confidence. For example, Table 6.7 yields, that with high confidence, SNoQE outperforms NFIQ 2.0 on FVC 2000 DB1, and NFIQ 2.0 outperforms SNoQE on FVC 2002 DB1 (both with bozorth3). For the other 8 databases paired with bozorth3, each quality estimator outperforms the other one an equal number of times, without statistical significance, however.

\subsubsection{SNoQE's Strengths and Weaknesses}

In addition to providing an important quality feature not sufficiently reflected by NFIQ and NFIQ 2.0, SNoQE is computationally also approximately up to five times faster than NFIQ 2.0, cf. Table 6.8.

By design, however, SNoQE can detect neither insufficient numbers of minutiae nor insufficient minutiae overlap. This is particularly the case for FVC 2002 DB1 with rather smudge-free prints, cf. Figure 6.10, some of which feature little overlap. This causes high FNMRs under high SNoQE quality, see Figure 6.8 and 6.9. The same holds true for other databases in which bad tail behavior of SNoQE can be found, exemplary are prints (c) and (d) of Figure 6.7 which have little overlap. 


\section{CHAPTER 7}

\section{Discussion and Outlook}

First and foremost, in this thesis a broad algorithm, cf. Algorithm 3.2.8, featuring a large set of tunable parameters, has been proposed for cartoon-residual decomposition. The new algorithm contains the known class of $\ell^{1}$-regularizations, as given in (1.3.2), as special cases, while also containing a variety of new cartoon-texture decompositions, to the best of the author's knowledge, not yet considered in the literature. Moreover, for Algorithm 3.2.8, the existence of fixed points, the convergence to a fixed point and the uniqueness of fixed points has been shown, each for specific families of input parameters, respectively. Moreover, in application a novel fingerprint quality feature, called SNoQE, based on measuring "smudge" noise, and a biometric quality validation scheme have been presented. Benefits and limitations of, as well as, future work on Algorithm 3.2.8 and SNoQE are discussed in the following.

\subsection{Cartoon-Residual Decompositions}

The proposed Algorithm 3.2.8, has been motivated by the limitation of loss-of-contrast in the classical $T V-\ell^{2}$-model, cf. Chapter 3.1.2, and its input parameters have been detailed in Chapter 3.2. It was shown that Algorithm 3.2.8 generalizes the ADMM algorithm for solving $\ell^{1}$-regularization problems and that the generalized feasibility Problem 3.2.9 is equivalent to solving a constrained double minimization, given in Problem 3.3.4, cf. Chapter 3.

We have introduced weak and strong admissibility, relating the input matrix-families $\underline{B}$ and $\underline{\widetilde{B}}$, as well as, eigenvalue conditions on $\underline{\mathfrak{C}}_{\underline{\underline{B}}}^{*} \underline{\mathfrak{C}}_{\underline{B}}$ and $\underline{\mathfrak{C}}_{\underline{B}} \underline{\mathfrak{C}}_{\underline{\underline{B}}}^{*}$ called the (CPC) and the relaxed (NEPC), cf. Definition 3.2.1. Given $(\underline{B}, \underline{\widetilde{B}})$ being weakly admissible and satisfying the (CPC) we have shown that the generalized Problem 3.2.9 has a solution, cf. Theorem 4.1.7, and hence, Algorithm 3.2.8 has a fixed point. Furthermore, we have shown that for $(\underline{B}, \underline{\widetilde{B}})$ being strongly admissible and satisfying the (NEPC) we can relate Problem 3.2.9 to a minimization problem. To take a step towards proving convergence in the case of weak admissibility we have given an alternative proof of 
convergence, not using the related minimization problem. Last, assuming that strong admissibility holds for the input matrix-families $(\underline{B}, \underline{\widetilde{B}})$ we have given an alternative proof for the convexity of the fixed point set and the existence of a unique fixed point of Algorithm 3.2.8 under the (NEPC) and the (CPC), respectively, in Theorem 4.3.3 and 4.3.5.

In Chapter 5, first numerical results show that the proposed Algorithm 3.2.8 features cartoon-residual decompositions that avoid loss-of-contrast and show promising results in denoising and separation of cartoon and texture. Moreover, the denoising experiments in Chapter 5.1 hinted at the fact that for correlated noise structures the generalized algorithm can provide a useful extension of the known class of $\ell^{1}$-problems, by using the new flexibility brought by weak admissibility. Furthermore, we showed that improvements over the classical $T V-\ell^{2}$ model are stable when parameters are trained beforehand and then tested on similar input images corrupted by noise matrices newly drawn from the same distribution. Last, we proposed wavelet-frame-based filters that showed to be superior in separating cartoon and texture over the classical $T V-\ell^{2}$-decomposition, exemplifying possible input filters for Algorithm 3.2.8.

In ongoing and future work, one focus is on employing machine learning tools to train the parameters of Algorithm 3.2.8 for specific purposes, say for denoising or, more applied, for smudge identification in fingerprints. On the one hand, comparison with state-of-the-art competitors in the respective fields could show the use of Algorithm 3.2.8 when employed in applications. On the other hand, analysis of the so trained parameters could yield heuristics for educated tuning of the parameters involved in Algorithm 3.2.8 in general.

Another focus is extending the convergence proof to the case of weak admissibility, which seems to be of particular interest, cf. Chapter 5. In particular, this means understanding how Lemma 4.2.4 can either be extended or replaced. Last, one might want to consider extending Algorithm 3.2.8 even further. One way would be to feature a continuous $\kappa \in[1,2]$, using a suitable homotopy from the anisotropic to the isotropic $\ell^{1}$ norm on $\Gamma_{P}$. Another one could lie in considering a change of the data-fidelity norm for the $T V-\ell^{2}$-problem as often proposed in the literature, cf. Chapter 1.4. This would mean assessing whether the proposed generalization of the algorithm given in [WT10] can be transferred to, for example, an algorithm that solves a $T V-G$ model, a model which is not equivalent to employing a matrix convolution in the data-fidelity term (Vese and Osher [VO03]). 


\subsection{Fingerprint Quality Estimation}

In Chapter 6 a new fingerprint quality feature was provided, that is based on the occurrence of smudge, and adopts a novel image decomposition technique for fingerprint quality estimation. Furthermore, a robust biometric quality validation scheme (RBQ VS) has been provided, that, with statistical significance, ranks the performance of different quality estimators, which is specifically apt for a conservative use-case. The resulting one-feature quality estimator SNoQE can compete with the popular NFIQ 2.0 and its previous version NFIQ, on the FVC databases and the NIST SD4 in terms of lowering error rates of comparison subsystems, whilst even outperforming the multifeature quality estimators on some databases.

Let us consider possible improvements of validation scheme and quality feature. Recall that due to minimizing the EER over a challenging database, we exclude considerably large numbers from matching. When excluding a large proportion of requests is not an option, at biometric border control for example, other objectives for the RBQ VS can be employed, for instance, using the lowest quality threshold for which a certain FAR, FRR or EER is reached. Also, additional penalization in Step (2.2.2/2.2.3), see Chapter 6.4, of the RBQ VS (see Chapter 6.4) taking in account the number of rejected prints could be included.

SNoQE was built by a specifically for fingerprints tailored cartoon-residual decomposition, the G3PD. Further parameter training for SNoQE as well as for G3PD (no longer optimizing for segmentation but for quality estimation) may further improve SNoQE. Alternatively, applying the proposed Algorithm 3.2.8, cf. Chapter 3, with trained input filters for the purpose of smudge and region-of-interest assessment might be considered. In order to obtain improvement beyond the presented results, training on much larger suitable databases is necessary.

Finally, recall that a naive combination of SNoQE and NFIQ 2.0 outperforms each single one. This hints towards a high potential for improvement by exploring more sophisticated combinations, for example adding minutiae quantity and overlap estimation and/or directly including the smudge noise quality feature in the NFIQ 2.0 random forest classifier. 
Appendices 



\section{CHAPTER A}

\section{Standard Results}

In this appendix proofs to the stated standard results are given for this thesis to be self-contained.

\section{A.1 Linear Algebra}

Lemma A.1.1 ([Bou89](II, $\S 10)) . \quad$ (i) Let $\mathfrak{M}$ be a linear operator on $\mathbb{C}^{n \times m}$, if its adjoint is given by $\mathfrak{M}^{*}=\left(N_{r, s}\right)_{r=0, s=0}^{n-1, m-1}$, we have

$$
N_{r, s}[k, \ell]=\overline{M_{k, \ell}[r, s]} .
$$

(ii) For any linear operator $\mathfrak{M}=\left(M_{r, s}\right)_{r=0, s=0}^{n-1, m-1}$ and $\mathfrak{N}=\left(N_{r, s}\right)_{r=0, s=0}^{n-1, m-1}$ on $\mathbb{C}^{n \times m}$ the operator $\mathfrak{M} \circ \mathfrak{N}$ is again a linear operator on $\mathbb{C}^{n \times m}$ with matrix entries $\left(L_{x, y}\right)_{x=0, y=0}^{n-1, m-1}$ given by

$$
L_{x, y}[k, \ell]=\sum_{r=0}^{n-1} \sum_{s=0}^{m-1} M_{x, y}[r, s] N_{r, s}[k, \ell],
$$

for all $0 \leq x, k \leq n-1$ and $0 \leq y, \ell \leq m-1$.

(iii) For any linear operators $\mathfrak{M}=\left(M_{r, s}\right)_{r=0, s=0}^{n-1, m-1}$ and $\mathfrak{N}=\left(N_{r, s}\right)_{r=0, s=0}^{n-1, m-1}$ on $\mathbb{C}^{n \times m}$ we have

$$
\left(\mathfrak{M}^{*} \mathfrak{N}\right)^{*}=\mathfrak{N}^{*} \mathfrak{M}
$$

(iv) Given a unitary linear operator $\mathfrak{N}=\left(N_{r, s}\right)_{r=0, s=0}^{n-1, m-1}$ on $\mathbb{C}^{n \times m}$, then we have

$$
\left\langle N_{r, s}, N_{k, \ell}\right\rangle=\delta_{r k} \delta_{s \ell},
$$

for all $0 \leq k, r \leq n-1$ and all $0 \leq \ell, s \leq m-1$. Furthermore,

$$
\mathfrak{N}\left(\overline{N_{r, s}}\right)=E_{r, s} \text { for all } 0 \leq r \leq n-1 \text { and } 0 \leq s \leq m-1 \text {. }
$$


(v) Given a linear operator $\mathfrak{M}$ on $\mathbb{C}^{n \times m}$ that is diagonalized by an unitary linear operator $\mathfrak{\Re}$ on $\mathbb{C}^{n \times m}$, let $\left(P_{r, s}\right)_{r=0, s=0}^{n-1, m-1}$ be defined by

$$
\left(P_{r, s}\right)_{r=0, s=0}^{n-1, m-1}:=\mathfrak{N} \mathfrak{M} \mathfrak{N}^{*},
$$

then

$$
P_{r, s}[r, s] \text { for } 0 \leq r \leq n-1 \text { and } 0 \leq s \leq m-1 \text {, }
$$

constitute all eigenvalues of $\mathfrak{M}$ to the eigenmatrices

$$
\overline{N_{r, s}} \text { for } 0 \leq r \leq n-1 \text { and } 0 \leq s \leq m-1 \text {, }
$$

respectively.

Proof. (i) We observe

$$
\begin{aligned}
& \langle\mathfrak{M}(A), B\rangle=\sum_{k=0}^{n-1} \sum_{\ell=0}^{m-1} \mathfrak{M}(A)[k, \ell] \overline{B[k, \ell]} \\
& =\sum_{k=0}^{n-1} \sum_{\ell=0}^{m-1} \sum_{r=0}^{n-1} \sum_{s=0}^{m-1} M_{k, \ell}[r, s] A[r, s] \overline{B[k, \ell]} \\
& =\sum_{r=0}^{n-1} \sum_{s=0}^{m-1} A[r, s] \overline{\sum_{k=0}^{n-1} \sum_{\ell=0}^{m-1} \overline{M_{k, \ell}[r, s]} B[k, \ell]} \\
& =\sum_{r=0}^{n-1} \sum_{s=0}^{m-1} A[r, s] \overline{\sum_{k=0}^{n-1} \sum_{\ell=0}^{m-1} N_{r, s}[k, \ell] B[k, \ell]}=\left\langle A, \mathfrak{M}^{*}(B)\right\rangle \text {. }
\end{aligned}
$$

(ii) Given a matrix $A \in \mathbb{C}^{n \times m}$ we have by construction

$$
\begin{aligned}
\mathfrak{M} \circ \mathfrak{N}(A) & =\mathfrak{M}\left(\left(\sum_{k=0}^{n-1} \sum_{\ell=0}^{m-1} N_{r, s}[k, \ell] A[k, \ell]\right)_{r=0, s=0}^{n-1, m-1}\right) \\
& =\left(\sum_{r=0}^{n-1} \sum_{s=0}^{m-1} M_{x, y}[r, s] \sum_{k=0}^{n-1} \sum_{\ell=0}^{m-1} N_{r, s}[k, \ell] A[k, \ell]\right)_{x=0, y=0}^{n-1, m-1} \\
& =\left(\sum_{k=0}^{n-1} \sum_{\ell=0}^{m-1}\left(\sum_{r=0}^{n-1} \sum_{s=0}^{m-1} M_{x, y}[r, s] N_{r, s}[k, \ell]\right) A[k, \ell]\right)_{x=0, y=0}^{n-1, m-1} .
\end{aligned}
$$

Hence, $\mathfrak{M} \circ \mathfrak{M}$ is again a linear operator on $\mathbb{C}^{n \times m}$ with matrix entries $\left(L_{x, y}\right)_{x=0, y=0}^{n-1, m-1}$ 
given by

$L_{x, y}[k, \ell]:=\sum_{r=0}^{n-1} \sum_{s=0}^{m-1} M_{x, y}[r, s] N_{r, s}[k, \ell]$ for all $0 \leq x, k \leq n-1$ and $0 \leq y, \ell \leq m-1$.

(iii) For the first part let $\left(L_{x, y}\right)_{x=0, y=0}^{n-1, m-1}$ be the matrix entries of the linear operator $\mathfrak{M}^{*} \mathfrak{N}$ on $\mathbb{C}^{n \times m}$ then we have by (i) and (ii) that

$$
\begin{aligned}
L_{x, y}[k, \ell] & =\sum_{r=0}^{n-1} \sum_{s=0}^{m-1} \overline{M_{r, s}[x, y]} N_{r, s}[k, \ell]=\overline{\sum_{r=0}^{n-1} \sum_{s=0}^{m-1} M_{r, s}[x, y] \overline{N_{r, s}[k, \ell]}} \\
& =\overline{L_{k, \ell}[x, y]},
\end{aligned}
$$

yielding the result via (i).

(iv) Let $\mathfrak{E}=\left(E_{r, s}\right)_{r=0, s=0}^{n-1, m-1}$ be the identity, then we have by (i) that

$$
\begin{aligned}
\mathfrak{N}^{*}\left(E_{r, s}\right) & =\left(\sum_{k=0}^{n-1} \sum_{\ell=0}^{m-1} \overline{N_{k, \ell}[x, y]} E_{r, s}[k, \ell]\right)_{x=0, y=0}^{n-1, m-1} \\
& =\left(\sum_{k=0}^{n-1} \sum_{\ell=0}^{m-1} \overline{N_{k, \ell}[x, y]} \delta_{r k} \delta_{s \ell}\right)_{x=0, y=0}^{n-1, m-1}=\overline{N_{r, s}} .
\end{aligned}
$$

This yields,

$$
\begin{aligned}
\left\langle N_{r, s}, N_{k, \ell}\right\rangle & =\left\langle\overline{\left\langle\mathfrak{N}^{*}\left(E_{r, s}\right)\right.}, \overline{\mathfrak{N}^{*}\left(E_{k, \ell}\right)}\right\rangle=\overline{\left\langle\mathfrak{N} \circ \mathfrak{N}^{*}\left(E_{k, \ell}\right), E_{r, s}\right\rangle} \\
& =\overline{\left\langle E_{k, \ell}, E_{r, s}\right\rangle}=\overline{\delta_{r k} \delta_{s \ell}}=\delta_{r k} \delta_{s \ell},
\end{aligned}
$$

where the third equality is due to $\mathfrak{R}$ being unitary. Lastly, we have

$$
\mathfrak{N}\left(\overline{N_{r, s}}\right)[x, y]=\operatorname{tr}\left(N_{x, y} N_{r, s}^{*}\right)=\left\langle N_{x, y}, N_{r, s}\right\rangle=\left\{\begin{array}{ll}
1 & \text { if }(x, y)=(r, s) \\
0 & \text { else }
\end{array} .\right.
$$

(v) Let $0 \leq r \leq n-1$ and $0 \leq s \leq m-1$ then, by $\mathfrak{N}$ being unitary and Remark (iv),

$$
\mathfrak{M}\left(\overline{N_{r, s}}\right)=\mathfrak{N}^{*} \mathfrak{N} \mathfrak{M} \mathfrak{N}^{*} \mathfrak{N}\left(\overline{N_{r, s}}\right)=\mathfrak{N}^{*} \mathfrak{N} \mathfrak{M} \mathfrak{N}^{*}\left(E_{r, s}\right),
$$

with $E_{r, s}$ defined as before. Hence,

$$
\mathfrak{M}\left(\overline{N_{r, s}}\right)=\mathfrak{N}^{*}\left(P_{r, s}[r, s] E_{r, s}\right)=P_{r, s}[r, s] \overline{N_{r, s}},
$$


where the first equation uses that $\mathfrak{M}$ is diagonalized by $\mathfrak{N}$ and the second one stems from $\mathfrak{R}$ being unitary, see (iv). By the pairwise orthogonality of the $N_{r, s}$ 's given by (iv) we found $n^{2}$ linearly independent eigenvectors, thus the above defined eigenvalues are all eigenvalues of $\mathfrak{M}$.

Remark A.1.2. (i) The DFT is a linear operator on $\mathbb{C}^{n \times m}$ given by $\mathfrak{F}=\left(F_{r, s}\right)_{r=0, s=0}^{n-1, m-1}$ with

$$
F_{r, s}[k, \ell]=\omega_{n}^{k r} \omega_{m}^{\ell s}=e^{-\frac{2 \pi i k r}{n}-\frac{2 \pi i \ell s}{m}},
$$

$$
\text { for } 0 \leq k \leq n-1 \text { and } 0 \leq \ell \leq m-1 \text { giving }
$$

$$
\widehat{A}=\mathfrak{F}(A) \quad \forall A \in \mathbb{C}^{n \times m}
$$

(ii) Note that $\mathfrak{F}$ is invertible, its inverse is given by the linear operator $\mathfrak{F}^{-1}=$ $\left(\widetilde{F}_{r, s}\right)_{r=0, s=0}^{n-1, m-1}$ with entries

$$
\widetilde{F}_{r, s}[k, \ell]=\frac{\overline{F_{r, s}[k, \ell]}}{n m}=\frac{\omega_{n}^{-k r} \omega_{m}^{-\ell s}}{n m} .
$$

Proof. (i) Follows by definition of the DFT.

(ii) By (ii) of Lemma 2.1.3 we have that

$$
\begin{aligned}
L_{x, y}[k, \ell] & =\sum_{r=0}^{n-1} \sum_{s=0}^{m-1} F_{x, y}[r, s] \frac{1}{n m} \widetilde{F}_{r, s}[k, \ell] \\
& =\frac{1}{n m} \sum_{r=0}^{n-1} \sum_{s=0}^{m-1} \omega_{n}^{r x} \omega_{m}^{s y} \omega_{n}^{-k r} \omega_{m}^{-\ell s} \\
& =\frac{1}{n m} \sum_{r=0}^{n-1} e^{\frac{2 \pi i r(x-k)}{n}} \sum_{s=0}^{m-1} e^{\frac{2 \pi i s(y-\ell)}{m}}=\delta_{x k} \delta_{y \ell} .
\end{aligned}
$$

Lemma A.1.3 ([SS03](Chap.7)). (i) The linear operator $\frac{1}{\sqrt{n m}} \mathfrak{F}$ is a unitary operator. In particular,

$$
\mathfrak{F}\left(\bar{F}_{r, s}\right)=\sqrt{n m} E_{r, s},
$$

for all $0 \leq r \leq n-1$ and $0 \leq s \leq m-1$.

(ii) Let $\mathfrak{C}_{A}$ be a matrix convolution and $\mathfrak{C}_{A}^{*}$ its adjoint with filters $A$ and $B$, respectively. Then

$$
\widehat{B}[k, \ell]=\overline{\widehat{A}[k, \ell]} .
$$


(iii) Let $\mathfrak{C}_{A}$ be a matrix convolution with filter $A$, then we have for any matrix $B \in \mathbb{C}^{n \times m}$

$$
\widehat{C_{A}(B)}=\widehat{A} \odot \widehat{B}
$$

(iv) Applying the discrete Fourier transform we diagonalize a matrix convolution. Additionally, any two matrix convolutions $\mathfrak{C}_{A}, \mathfrak{C}_{B}: \mathbb{C}^{n \times m} \rightarrow \mathbb{C}^{n \times m}$ commute, i.e.

$$
\mathfrak{C}_{A} \circ \mathfrak{C}_{B}(L)=\mathfrak{C}_{B} \circ \mathfrak{C}_{A}(L)
$$

for all matrices $L \in \mathbb{C}^{n \times m}$.

Proof. (i) Let $\left(F_{r, s}^{\operatorname{Inv}}\right)_{r=0, s=0}^{n-1, m-1}$ be the matrix entries of $\mathfrak{F}^{-1}$, we have that

$$
\sqrt{n m} F_{r, s}^{\mathrm{Inv}}[k, \ell]=\frac{1}{\sqrt{n m}} \overline{F_{r, s}[k, \ell]}=\frac{1}{\sqrt{n m}} \omega_{n}^{-k r} \omega_{m}^{-\ell s}=\frac{1}{\sqrt{n m}} \overline{F_{k, \ell}[r, s]},
$$

hence, $\sqrt{n m} \mathfrak{F}^{-1}$ is the adjoint of $\frac{1}{\sqrt{n m}} \mathfrak{F}$, by (ii) of Remark 2.1.7. Moreover, $\sqrt{n m} \mathfrak{F}^{-1}$ is also its inverse, yielding,

$$
\frac{1}{\sqrt{n m}} \mathfrak{F} \circ\left(\frac{1}{\sqrt{n m}} \mathfrak{F}\right)^{*}=\mathfrak{E} .
$$

By (iii) of Lemma 2.1.3 we obtain

$$
\mathfrak{F}\left(\bar{F}_{r, s}\right)=\sqrt{n m} E_{r, s},
$$

for all $0 \leq r \leq n-1$ and $0 \leq s \leq m-1$.

(ii) By (i) of Lemma 2.1.3 we have that

$$
B[k, \ell]=\overline{A[-k,-\ell]} .
$$

Furthermore, we see that

$$
\begin{aligned}
& \widehat{B}[k, \ell]=\sum_{r=0}^{n-1} \sum_{s=0}^{m-1} \overline{A[-r,-s]} \omega_{n}^{k r} \omega_{m}^{\ell s} \\
& =\sum_{r=0}^{n-1} \sum_{s=0}^{m-1} \overline{A[r, s]} \omega_{n}^{-k r} \omega_{m}^{-\ell s} \\
& =\overline{\sum_{r=0}^{n-1} \sum_{s=0}^{m-1} A[r, s] \omega_{n}^{k r} \omega_{m}^{\ell s}}=\overline{\widehat{A}[k, \ell]} .
\end{aligned}
$$


(iii) For $\omega_{t}:=e^{-\frac{2 \pi i}{t}}$ we have

$$
\begin{aligned}
\widehat{\mathfrak{S}_{A}(B)}[k, \ell] & =\sum_{r=0}^{n-1} \sum_{s=0}^{m-1} \sum_{p=0}^{n-1} \sum_{q=0}^{m-1} A[r-p, s-q] B[p, q] \omega_{n}^{k r} \omega_{m}^{\ell s} \\
& =\sum_{r=0}^{n-1} \sum_{s=0}^{m-1} \sum_{p=0}^{n-1} \sum_{q=0}^{m-1} A[r-p, s-q] \omega^{k(r-p)+\ell(s-q)} B[p, q] \omega_{n}^{k p} \omega_{m}^{\ell q} \\
& =\sum_{p=0}^{n-1} \sum_{q=0}^{m-1} B[p, q] \omega_{n}^{k p} \omega_{m}^{\ell q}\left(\sum_{r=0}^{n-1} \sum_{s=0}^{m-1} A[r-p, s-q] \omega_{n}^{k(r-p)} \omega_{m}^{\ell(s-q)}\right) \\
& =\sum_{p=0}^{n-1} \sum_{q=0}^{m-1} B[p, q] \omega_{n}^{k p} \omega_{m}^{\ell q}\left(\sum_{t_{1}=0}^{n-1} \sum_{t_{2}=0}^{m-1} A\left[t_{1}, t_{2}\right] \omega_{n}^{k t_{1}} \omega_{m}^{\ell t_{2}}\right) \\
& =(\widehat{A} \odot \widehat{B})[k, \ell] .
\end{aligned}
$$

(iv) Follows at once.

Lemma A.1.4. (i) Let $\underline{\mathfrak{C}}_{\underline{B}}$ be a matrix-family convolution defined as above then its adjoint is given by

$$
\left(\underline{\mathfrak{C}}_{\underline{B}}\right)^{*}: \Gamma_{P} \rightarrow \mathbb{R}^{n \times m} ; \quad \underline{W} \mapsto \sum_{p=1}^{P} \mathfrak{C}_{B_{p}}^{*}\left(W_{p}\right) .
$$

(ii) Let $\underline{\underline{\mathfrak{C}}}_{C}$ be an entry-wise matrix-family convolution defined as above then its adjoint is given by

$$
\left(\underline{\underline{C}}_{\underline{C}}\right)^{*}: \Gamma_{P} \rightarrow \Gamma_{P} ; \quad \underline{W} \mapsto\left(\mathfrak{C}_{C_{1}}^{*}\left(W_{1}\right), \ldots \mathfrak{C}_{C_{P}}^{*}\left(W_{P}\right)\right)
$$

Proof. (i)

$$
\begin{aligned}
\left\langle\underline{W}, \underline{\mathfrak{C}}_{\underline{B}}(U)\right\rangle & =\sum_{p=1}^{P}\left\langle W_{p}, \mathfrak{C}_{B_{p}}(U)\right\rangle \\
& =\sum_{p=1}^{P}\left\langle\mathfrak{C}_{B_{p}}^{*}\left(W_{p}\right), U\right\rangle \\
& =\left\langle\sum_{p=1}^{P} \mathfrak{C}_{B_{p}}^{*}\left(W_{p}\right), U\right\rangle .
\end{aligned}
$$


(ii)

$$
\begin{aligned}
\left\langle\underline{\underline{W}}, \underline{\underline{\mathfrak{C}}}_{\underline{C}}(\underline{V})\right\rangle & =\sum_{p=1}^{P}\left\langle W_{p}, \mathfrak{C}_{C_{p}}\left(V_{p}\right)\right\rangle \\
& =\sum_{p=1}^{P}\left\langle\mathfrak{C}_{C_{p}^{*}}^{*}\left(W_{p}\right), V_{p}\right\rangle .
\end{aligned}
$$

Lemma A.1.5 (Plancherel). Let $A, B \in \mathbb{C}^{n \times m}$, then we have

$$
\langle A, B\rangle=n m\langle\widehat{A}, \widehat{B}\rangle .
$$

Proof.

$$
\begin{aligned}
\langle\widehat{A}, \widehat{B}\rangle & =\sum_{k=0}^{n-1} \sum_{\ell=0}^{m-1} \widehat{A}[k, \ell] \overline{\widehat{B}[k, \ell]} \\
& =\sum_{k=0}^{n-1} \sum_{\ell=0}^{m-1}\left(\sum_{r=0}^{n-1} \sum_{s=0}^{m-1} A[r, s] \omega_{n}^{k r} \omega_{m}^{\ell s}\right) \overline{\left(\sum_{p=0}^{n-1} \sum_{q=0}^{m-1} B[p, q] \omega_{n}^{k p} \omega_{m}^{\ell q}\right)} \\
& =\sum_{k=0}^{n-1} \sum_{\ell=0}^{m-1} \sum_{r=0}^{n-1} \sum_{s=0}^{m-1} \sum_{p=0}^{n-1} \sum_{q=0}^{m-1} A[r, s] \overline{B[p, q]} \omega_{n}^{k(r-p)} \omega_{m}^{\ell(s-q)} \\
& =\sum_{r=0}^{n-1} \sum_{s=0}^{m-1} \sum_{p=0}^{n-1} \sum_{q=0}^{m-1} A[r, s] \overline{B[p, q]} \sum_{k=0}^{n-1} \sum_{\ell=0}^{m-1} \omega_{n}^{k(r-p)} \omega_{m}^{\ell(s-q)} \\
& =n m \sum_{t_{1}=0}^{n-1} \sum_{t_{2}=0}^{m-1} A\left[t_{1}, t_{2}\right] \overline{B\left[t_{1}, t_{2}\right]}
\end{aligned}
$$

since $\sum_{k=0}^{n-1} c \omega_{n}^{k p}=0$ for all $p \neq 0$ and $n \in \mathbb{N}$.

\section{A.2 Convex Analysis}

Proposition A.2.1 ([ET99](p.38,Prop.2.2)). Let $\mathcal{F}=\mathcal{F}_{1}+\mathcal{F}_{2}: \mathbb{R}^{n} \rightarrow \mathbb{R}$ be a coercive functional, with $\mathcal{F}_{2}$ lower semi-continuous and convex. Furthermore, let $\mathcal{F}_{1}$ be convex and differentiable with gradient $D_{y} \mathcal{F}_{1}$ at a point $y \in \mathbb{R}^{n}$. Then we have

$$
u \in \arg \min _{u \in \mathbb{R}^{n}} \mathcal{F}(u),
$$

if and only if the following inequality holds

$$
\left\langle D_{u} \mathcal{F}_{1}, v-u\right\rangle+\mathcal{F}_{2}(v)-\mathcal{F}_{2}(u) \geq 0, \quad \forall v \in \mathbb{R}^{n}
$$


Proof. This proof is a reproduction of [ET99][p.38,Prop.2.2]. Let $u \in \arg \min _{u \in \mathbb{R}^{n}} \mathcal{F}(u)$ then we have

$$
\mathcal{F}(u)-\mathcal{F}((1-\xi) u+\xi v) \leq 0, \text { for all } \xi \in[0,1] \text { and } v \in \mathbb{R}^{n}
$$

By convexity of $\mathcal{F}_{2}$ we can rewrite this to

$\mathcal{F}_{1}(u)-\mathcal{F}_{1}((1-\xi) u+\xi v)+\mathcal{F}_{2}(u)-(1-\xi) \mathcal{F}_{2}(u)-\xi \mathcal{F}_{2}(v) \leq 0$, for all $\xi \in[0,1]$ and $v \in \mathbb{R}^{n}$

Multiply with $-\frac{1}{\xi}$ and simplify to obtain

$$
\frac{\mathcal{F}_{1}(u+\xi(v-u))-\mathcal{F}_{1}(u)}{\xi}+\mathcal{F}_{2}(v)-\mathcal{F}_{2}(u) \geq 0, \text { for all } \xi \in[0,1] \text { and } v \in \mathbb{R}^{n}
$$

If we now let $\xi \rightarrow 0$ we obtain precisely (4.2.1).

Conversely, by convexity of $\mathcal{F}_{1}$ we have for all $v \in \mathbb{R}^{n}$ that

$$
\mathcal{F}_{1}(v)-\mathcal{F}_{1}(u)-\left\langle D_{u} \mathcal{F}_{1}, v-u\right\rangle \geq 0
$$

Together, with (4.2.1) yields that $u$ is a minimizer of $\mathcal{F}$.

Lemma A.2.2 ([WT10][The.4.1],[Ber82][p.96ff]). Let $F \in \mathbb{R}^{n \times m}, \underline{B} \in \Gamma_{P}, \mu \in \mathbb{R}_{+}$and $\kappa \in\{1,2\}$. Then $U^{\dagger} \in \mathbb{R}^{n \times m}$ is a solution of Problem 3.1.2 if and only if there exists $\underline{W}^{\dagger} \in \Gamma_{P}$ and $\underline{\lambda}^{\dagger} \in \Gamma_{P}$ such that $\left(U^{\dagger}, \underline{W}^{\dagger}, \underline{\lambda}^{\dagger}\right)$ is a saddlepoint of (3.1.2).

Proof. For convenience we reproduce and generalize here the proof of Theorem 4.1 in [WT10].

Suppose $\left(U^{\dagger}, \underline{W}^{\dagger}, \underline{\lambda}^{\dagger}\right) \in \Gamma_{1+2 P}$ is a solution of Problem 3.1.3, then we have by the first inequality of (3.1.3) that

$$
\underline{\mathfrak{C}}_{\underline{B}}\left(U^{\dagger}\right)=\underline{W}^{\dagger} .
$$

The above equation together with the second inequality in (3.1.3) shows that $U^{\dagger}$ is a solution of Problem 3.1.2.

For the converse let now $U^{\dagger} \in \mathbb{R}^{n \times m}$ be a solution of Problem 3.1.2. Choose $\underline{W}^{\dagger}=$ $\underline{\widetilde{C}}_{\underline{B}}\left(U^{\dagger}\right)$. Define the functional

$$
Q_{2}: \mathbb{R}^{n \times m} \rightarrow \mathbb{R}, \quad U \mapsto\left|\underline{\mathfrak{C}}_{\underline{B}}(U)\right|_{1, \kappa},
$$

this is lower semi-continuous and convex, while

$$
Q_{1}:=\|F-U\|^{2},
$$


is convex and differentiable, so we can use Proposition 2.2.2 from [ET99][p.38,Prop.2.2] to show

$$
\mu\left\langle U^{\dagger}-F, U-U^{\dagger}\right\rangle+Q_{2}(U)-Q_{2}\left(U^{\dagger}\right) \geq 0 .
$$

Now by Remark 3.2 in [ET99][p.45] we have that

$$
\mu\left(F-U^{\dagger}\right) \in \partial Q_{2}\left(U^{\dagger}\right)
$$

where $\partial Q_{2}\left(U^{\dagger}\right)$ is the subdifferential of $Q_{2}$ at $U^{\dagger}$, see Definition 2.2.1. Now, by subdifferential calculus, see Proposition 5.7 in [ET99][p.27], we obtain for

$$
\widetilde{Q}_{2}: \Gamma_{P} \rightarrow \mathbb{R}, \quad \underline{W} \mapsto\|\underline{W}\|_{1, \kappa},
$$

that

$$
\mu\left(F-U^{\dagger}\right) \in \underline{\mathfrak{C}}_{\underline{B}}^{*}\left(\partial \widetilde{Q}_{2}\left(\underline{\mathfrak{C}}_{\underline{B}}\left(U^{\dagger}\right)\right)\right) .
$$

So we can choose $\underline{\lambda}^{\dagger} \in \Gamma_{P}$ such that

$$
-\underline{\mathbb{C}}_{\underline{B}}^{*}\left(\underline{\lambda}^{\dagger}\right)=\mu\left(F-U^{\dagger}\right) \quad \text { and } \quad-\underline{\lambda}^{\dagger} \in \partial \widetilde{Q}_{2}\left(\underline{\mathfrak{C}}_{\underline{B}}\left(U^{\dagger}\right)\right)
$$

Let us verify that $\left(U^{\dagger}, \underline{W}^{\dagger}, \underline{\lambda}^{\dagger}\right)$ is a saddle-point of (3.1.2). By $\underline{\mathfrak{c}}_{\underline{B}}\left(U^{\dagger}\right)=\underline{W}^{\dagger}$, the first equality is satisfied. To show the second inequality let us first recall the definition of subdifferentiability and use (2.2.1) for $\underline{\lambda}^{\dagger}$ to obtain

$$
|\underline{W}|_{1, \kappa}-\left|\underline{W}^{\dagger}\right|_{1, \kappa}+\left\langle\underline{\lambda^{\dagger}}, \underline{W}-\underline{W}^{\dagger}\right\rangle \geq 0
$$

for all $\underline{W} \in \Gamma_{P}$. Yielding,

$$
\underbrace{|\underline{W}|_{1, \kappa}-\left|\underline{W^{\dagger}}\right|_{1, \kappa}+\left\langle\underline{\lambda}^{\dagger}, \underline{W}-\underline{W^{\dagger}}\right\rangle}_{\geq 0}+\beta\langle\underbrace{\underline{W}^{\dagger}-\underline{\mathfrak{c}}_{\underline{B}}\left(U^{\dagger}\right)}_{=0}, \underline{W}-\underline{W^{\dagger}}\rangle \geq 0,
$$

for all $\underline{W} \in \Gamma_{P}$. Hence, by Proposition 2.2.2 we have that for fixed $U^{\dagger} \in \mathbb{R}^{n \times m}$ and $\underline{\lambda}^{\dagger} \in \Gamma_{P}$ the minimizer of $\mathcal{J}_{\mathrm{AL}}$ is given by $\underline{W}^{\dagger}$. Regarding $U^{\dagger}$ calculate for all $U \in \mathbb{R}^{n \times m}$ that

$$
\begin{aligned}
\frac{\mu}{2}\left\|U-F-\left(U^{\dagger}-F\right)\right\|^{2} & \geq 0 \\
\Leftrightarrow \quad \frac{\mu}{2}\left(\|U-F\|^{2}+\left\|U^{\dagger}-F\right\|^{2}\right) & \geq \mu\left\langle U-F, U^{\dagger}-F\right\rangle . \\
\Leftrightarrow \quad \frac{\mu}{2}\|U-F\|^{2}-\frac{\mu}{2}\left\|U^{\dagger}-F\right\|^{2} \geq \mu\left\langle U-F-U^{\dagger}+F, U^{\dagger}-F\right\rangle & \Leftrightarrow \quad \frac{\mu}{2}\|U-F\|^{2}-\frac{\mu}{2}\left\|U^{\dagger}-F\right\|^{2} \geq\left\langle U-U^{\dagger}, \underline{\mathfrak{C}}_{\underline{B}}^{*}\left(\underline{\lambda}^{\dagger}\right)\right\rangle,
\end{aligned}
$$


so we obtain,

$\underbrace{\frac{\mu}{2}\|U-F\|_{2}^{2}-\frac{\mu}{2}\left\|U^{\dagger}-F\right\|^{2}-\left\langle\underline{\mathfrak{C}}_{\underline{B}}^{*}\left(\underline{\lambda}^{\dagger}\right), U-U^{\dagger}\right\rangle}_{\geq 0}-\beta\langle\underbrace{\left\langle\underline{\mathfrak{C}}_{\underline{B}}^{*}\left(\underline{W}^{\dagger}-\underline{\mathfrak{C}}_{\underline{B}}\left(U^{\dagger}\right)\right)\right.}_{=0}, U-U^{\dagger}\rangle \geq 0$,

for all $U \in \mathbb{R}^{n \times m}$. By Proposition 2.2.2 we conclude that for fixed $\underline{W}^{\dagger}$ and $\underline{\lambda}^{\dagger}$ the minimizer of $\mathcal{J}_{\mathrm{AL}}$ is given by $U^{\dagger}$, finishing the proof.

Lemma A.2.3 ([WT10][The.4.4]). Let $F \in \mathbb{R}^{n \times m}, \underline{B} \in \Gamma_{P}, \kappa \in\{1,2\}$ and $\beta \in \mathbb{R}_{+}$. A point $\left(U^{\dagger}, \underline{W}^{\dagger}, \underline{\lambda}^{\dagger}\right) \in \Gamma_{1+2 P}$ is a fixed point of Algorithm 3.1 .5 if and only if $\left(U^{\dagger}, \underline{W^{\dagger}}, \underline{\lambda}^{\dagger}\right)$ is a saddlepoint of (3.1.2).

Proof. We present a proof of Lemma 3.1.6, which is a direct consequence of Theorem 4.4 in [WT10], using our notation.

Let $\left(U^{\dagger}, \underline{W}^{\dagger}, \underline{\lambda}^{\dagger}\right) \in \Gamma_{1+2 P}$ be a solution of Problem 3.1.3, then defining $\left(U^{(0)}, \underline{W}^{(0)}, \underline{\lambda}^{(1)}\right):=$ $\left(U^{\dagger}, \underline{W}^{\dagger}, \underline{\lambda}^{\dagger}\right)$ we have by the second inequality of (3.1.3) that $\underline{W}^{(1)}=\underline{W}^{(0)}$ and $U^{(1)}=$ $U^{(0)}$. By the first inequality we obtain

$$
\underline{\widetilde{c}}_{\underline{B}}\left(U^{\dagger}\right)=\underline{W}^{\dagger},
$$

hence, $\underline{\lambda}^{(2)}=\underline{\lambda}^{(1)}$, making $\left(U^{\dagger}, \underline{W}^{\dagger}, \underline{\lambda}^{\dagger}\right)$ a fixed point of Algorithm 3.1.5.

Conversely let $\left(U^{\dagger}, \underline{W}^{\dagger}, \underline{\lambda}^{\dagger}\right) \in \Gamma_{1+2 P}$ be a fixed point of Algorithm 3.1.5. Then, by the third updating step we have,

$$
\underline{\mathfrak{c}}_{\underline{B}}\left(U^{\dagger}\right)=\underline{W}^{\dagger},
$$

hence,

$$
J_{\mathrm{AL}}\left(U^{\dagger}, \underline{W^{\dagger}}, \underline{\lambda}^{\dagger}\right)=J_{\mathrm{AL}}\left(U^{\dagger}, \underline{W}^{\dagger}, \underline{\lambda}\right),
$$

for all $\underline{\lambda} \in \Gamma_{P}$, yielding the first inequality of (3.1.3).

The first two updating-steps yield the following inequalities by Proposition 2.2.2: With

$$
\mathcal{F}_{1}(\underline{W}):=\frac{\beta}{2}|| \underline{W}-\underline{\underline{C}}_{\underline{B}}(U)||^{2}, \quad \mathcal{F}_{2}(\underline{W}):=|\underline{W}|_{1, \kappa}+\langle\underline{\lambda}, \underline{W}\rangle
$$

we obtain,

$$
\beta\left\langle\underline{W}^{\dagger}-\underline{\mathfrak{C}}_{\underline{B}}\left(U^{\dagger}\right), \underline{W}-\underline{W}^{\dagger}\right\rangle+|\underline{W}|_{1, \kappa}-\left|\underline{W^{\dagger}}\right|_{1, \kappa}+\left\langle\underline{\lambda}, \underline{W}-\underline{W^{\dagger}}\right\rangle \geq 0,
$$

for all $\underline{W} \in \Gamma_{P}$. While via

$$
\mathcal{F}_{1}(U):=\frac{\beta}{2}\left\|\underline{W}-\underline{\mathfrak{C}}_{\underline{B}}(U)\right\|^{2}, \quad \mathcal{F}_{2}(U):=\frac{\mu}{2}\|F-U\|^{2}+\left\langle\underline{\lambda}, \underline{\mathfrak{C}}_{\underline{B}}(U)\right\rangle,
$$


we obtain

$$
\begin{aligned}
\beta\left\langle\underline{\mathfrak{C}}_{\underline{B}}^{*} \underline{\mathfrak{C}}_{\underline{B}}\left(U^{\dagger}\right)-\underline{\mathfrak{C}}_{\underline{B}}^{*}\left(\underline{W}^{\dagger}\right), U-U^{\dagger}\right\rangle & +\frac{\mu}{2}\|U-F\|^{2} \\
& -\frac{\mu}{2}\left\|U^{\dagger}-F\right\|^{2}-\left\langle\underline{\lambda}, \underline{\mathfrak{C}}_{\underline{B}}\left(U-U^{\dagger}\right)\right\rangle \geq 0,
\end{aligned}
$$

for all $U \in \mathbb{R}^{n \times m}$. So by (A.2.6) we have

$$
\begin{aligned}
|\underline{W}|_{1, \kappa}+\left\langle\underline{\lambda}, \underline{W}-\underline{W}^{\dagger}\right\rangle & \geq\left|\underline{W}^{\dagger}\right|_{1, \kappa}, \\
\frac{\mu}{2}\|U-F\|^{2}-\left\langle\underline{\lambda}, \underline{\mathfrak{c}}_{\underline{B}}\left(U-U^{\dagger}\right)\right\rangle & \geq \frac{\mu}{2} \| U^{\dagger}-\left.F\right|^{2},
\end{aligned}
$$

for all $(U, \underline{W}) \in \Gamma_{1+P}$. Adding the above we obtain

$$
|\underline{W}|_{1, \kappa}+\frac{\mu}{2}\|U-F\|^{2}+\left\langle\underline{\lambda}, \underline{W}-\underline{\mathfrak{c}}_{\underline{B}}(U)\right\rangle \geq\left|\underline{W^{\dagger}}\right|_{1, \kappa}+\frac{\mu}{2}\left\|U^{\dagger}-F\right\|^{2},
$$

for all $(U, \underline{W}) \in \Gamma_{1+P}$, yielding using again (A.2.6) the second inequality of (3.1.3), finishing the proof.

Lemma A.2.4 ([BC11],[BV04]). (i) For a given $U \in \mathbb{R}^{n \times m}, \underline{B}, \underline{\lambda} \in \Gamma_{P}, \kappa \in\{1,2\}$, as well as $\beta \in \mathbb{R}_{+}$, the minimizer of

$$
\mathcal{J}_{1}(\underline{W}):=|\underline{W}|_{1, \kappa}+\frac{\beta}{2} \| \underline{W}-\left.\underline{\mathfrak{C}}_{\underline{B}}(U)\right|^{2}+\langle\underline{\lambda}, \underline{W}\rangle
$$

is given by

$$
\underline{W}^{\dagger}=\mathrm{S}_{\kappa}\left(\underline{\mathfrak{c}}_{\underline{B}}(U)-\frac{1}{\beta} \underline{\lambda} ; \frac{1}{\beta}\right),
$$

where $S_{\kappa}: \Gamma_{P} \rightarrow \Gamma_{P}$ is the isotropic $(\kappa=2)$ or anisotropic $(\kappa=1)$ soft-shrinkage function, see Definition 2.1.17.

(ii) For a given $F \in \mathbb{R}^{n \times m}, \underline{B}, \underline{W}, \underline{\lambda} \in \Gamma_{P}$, and $\beta \in \mathbb{R}_{+}$, the minimizer of

$$
\mathcal{J}_{2}(U):=\frac{\mu}{2}\|F-U\|^{2}+\frac{\beta}{2}\left\|\underline{W}-\underline{\mathfrak{C}}_{\underline{B}}(U)\right\|^{2}-\left\langle\underline{\lambda}, \underline{\mathfrak{C}}_{\underline{B}}(U)\right\rangle,
$$

is given by

$$
U^{\dagger}=\mu\left(\mu \mathfrak{E}+\beta\left(\underline{\mathfrak{C}}_{\underline{B}}^{*} \underline{\mathfrak{C}}_{\underline{B}}\right)\right)^{-1}(F)+\left(\mu \mathfrak{E}+\beta\left(\underline{\mathfrak{C}}_{\underline{B}}^{*} \underline{\mathfrak{C}_{B}}\right)\right)^{-1} \underline{\mathfrak{C}}_{\underline{B}}^{*}\left(\underline{W}+\frac{1}{\beta} \underline{\lambda}\right) .
$$

Proof. $\quad$ (i) Minimizing $\mathcal{J}_{1}$ w.r.t. $\underline{W}$ we first define

$$
\mathrm{A}_{\kappa}(\underline{W}):=|\underline{W}|_{1, \kappa}+\langle\underline{\lambda}, \underline{W}\rangle,
$$


and the prox-operator as done in [BC11][p.175,Def.12.23] by

$$
\operatorname{prox}_{1 / \beta A_{\kappa}}\left(\underline{\mathfrak{C}}_{\underline{B}}(U)\right):=\arg \min _{\underline{W} \in \Gamma_{P}}\left(\frac{1}{\beta} \mathrm{A}_{\kappa}(\underline{W})+\frac{1}{2}\left\|\underline{W}-\underline{\mathfrak{C}}_{\underline{B}}(U)\right\|^{2}\right),
$$

for $\kappa=1,2$.

Case 1: $\kappa=1$ :

The set-valued subdifferential of $\mathrm{A}$ at $\underline{W}$ is given by

$$
\begin{aligned}
& \partial \mathrm{A}_{1}(\underline{W})=\left\{\underline{V} \in \Gamma_{P}:\right. \\
& V_{p}[k, \ell] \begin{cases}=1+\lambda_{p}[k, \ell] & \text { if } W_{p}[k, \ell]>0 \\
\in\left[-1+\lambda_{p}[k, \ell], 1+\lambda_{p}[k, \ell]\right] & \text { if } \left.W_{p}[k, \ell]=0, \text { for all } k, \ell, p\right\}, \\
=-1+\lambda_{p}[k, \ell] & \text { if } W_{p}[k, \ell]<0\end{cases}
\end{aligned}
$$

see Example 3.4 in [HUL93a][p.260f].For calculating the minimizer of $\mathcal{J}_{1}$ we use Proposition 16.34 of [BC11][p.233] stating

$$
\underline{W}^{\dagger}=\operatorname{prox}_{1 / \beta A_{1}}\left(\underline{\mathfrak{C}}_{\underline{B}}(U)\right) \quad \Leftrightarrow \quad \underline{\mathfrak{C}}_{\underline{B}}(U) \in\left(\underline{\underline{\mathfrak{E}}}+\frac{1}{\beta} \partial \mathrm{A}_{1}\right)\left(\underline{W}^{\dagger}\right),
$$

where $\underline{\underline{E}}$ is considered in this case to be the set-valued mapping

$$
\underline{\underline{E}}: \underline{W} \rightrightarrows\{\underline{W}\}
$$

Then to compute any $\underline{W}^{\dagger}$ we consider the possibly set-valued mapping ( $\underline{\underline{\mathfrak{E}}}+$ $\left.1 / \beta \partial \mathrm{A}_{1}\right)^{-1}$ defined by

$$
\left(\underline{\underline{\mathfrak{E}}}+1 / \beta \partial \mathrm{A}_{1}\right)^{-1}(\underline{V})=\left\{\underline{V^{0}} \in \Gamma_{P}: \exists \underline{V}^{1} \in \partial \mathrm{A}_{1}\left(\underline{V}^{0}\right) \text { such that } \underline{V}^{0}+1 / \beta \underline{V^{1}}=\underline{V} \cdot\right\}
$$

For an entry $(k, \ell, p)$ of $\underline{V^{0}} \in\left(\underline{\underline{\mathfrak{E}}}+1 / \beta \partial A_{1}\right)^{-1}(\underline{V})$ we have

$$
V_{p}^{0}[k, \ell]= \begin{cases}V_{p}[k, \ell]-\frac{1}{\beta}-\frac{1}{\beta} \lambda_{p}[k, \ell] & \text { if } V_{p}[k, \ell]-\frac{1}{\beta} \lambda_{p}[k, \ell]>\frac{1}{\beta} \\ 0 & \text { if } V_{p}[k, \ell]-\frac{1}{\beta} \lambda_{p}[k, \ell] \in\left[-\frac{1}{\beta}, \frac{1}{\beta}\right] . \\ V_{p}[k, \ell]+\frac{1}{\beta}-\frac{1}{\beta} \lambda_{p}[k, \ell] & \text { if } V_{p}[k, \ell]-\frac{1}{\beta} \lambda_{p}[k, \ell]<\frac{1}{\beta}\end{cases}
$$

Hence, $\left(\underline{\underline{\mathfrak{E}}}+1 / \beta \partial \mathrm{A}_{1}\right)^{-1}$ is single-valued. Plugging $\left(\underline{\underline{\mathfrak{E}}}+1 / \beta \partial \mathrm{A}_{1}\right)^{-1}$ into (A.2.9) we have

$$
\underline{W}^{\dagger}=\mathrm{S}_{1}\left(\underline{\underline{c}}_{\underline{B}}(U)-\frac{1}{\beta} \underline{\lambda} ; \frac{1}{\beta}\right) .
$$


Case 2: $\kappa=2$ :

We have that the set-valued subdifferential of $A_{2}$ at $\underline{W}$ is given by

$$
\begin{aligned}
& \qquad \mathrm{A}_{2}(\underline{W})=\left\{\underline{V} \in \Gamma_{P} \text { : for all } k, \ell\right. \text { we have: } \\
& \text { If }\left\|\left(W_{p}[k, \ell]\right)_{p=1}^{P}\right\|>0 \text { then }\left(V_{p}[k, \ell]\right)_{p=1}^{P}=\frac{\left(W_{p}[k, \ell]\right)_{p=1}^{P}}{\left\|\left(W_{p}[k, \ell]\right)_{p=1}^{P}\right\|}+\left(\lambda_{p}[k, \ell]\right)_{p=1}^{P}, \\
& \text { else } \left.\left\|\left(W_{p}[k, \ell]\right)_{p=1}^{P}\right\|=0 \text {, then }\left(V_{p}[k, \ell]\right)_{p=1}^{P} \in \mathcal{B}_{1}\left(\left(\lambda_{p}[k, \ell]\right)_{p=1}^{P}\right)\right\}
\end{aligned}
$$

where $\mathcal{B}_{1}(x)$ is the ball of radius 1 around $x \in \mathbb{R}^{P}$. Using again Proposition 16.34 of [BC11][p.233,Prop.16.34] we obtain

$$
\underline{W}^{\dagger}=\operatorname{prox}_{1 / \beta \mathrm{A}_{2}}\left(\underline{\mathfrak{C}}_{\underline{B}}(U)\right) \quad \Leftrightarrow \quad \underline{\mathfrak{C}}_{\underline{B}}(U) \in\left(\underline{\underline{\mathfrak{E}}}+\frac{1}{\beta} \partial \mathrm{A}_{2}\right)\left(\underline{W}^{\dagger}\right),
$$

where $\underline{\underline{E}}$ is again considered to be set-valued. Computing again the possibly set-valued mapping $\left(\underline{\underline{\mathfrak{E}}}+1 / \beta \partial \mathrm{A}_{2}\right)^{-1}$ defined by

$$
\left(\underline{\underline{\mathfrak{E}}}+1 / \beta \partial \mathrm{A}_{2}\right)^{-1}(\underline{V})=\left\{\underline{V}^{0} \in \Gamma_{P}: \exists \underline{V^{1}} \in \partial \mathrm{A}_{2}\left(\underline{V}^{0}\right) \text { such that } \underline{V}^{0}+1 / \beta \underline{V^{1}}=\underline{V} \cdot\right\}
$$

Consider for fixed $0 \leq k \leq n-1$ and $0 \leq \ell \leq m-1$ the vector $x:=\left(V_{p}[k, \ell]-\right.$ $\left.\lambda_{p}[k, \ell]\right)_{p=1}^{P}$ and $\underline{V}^{0} \in\left(\underline{\underline{\mathfrak{E}}}+1 / \beta \partial A_{1}\right)^{-1}(\underline{V})$ then we have for $\|x\|>0$ that

$$
\left(V_{p}^{0}[k, \ell]\right)_{p=1}^{P}=\left(\left\|\left(V_{p}[k, \ell]\right)_{p=1}^{P}\right\|-\frac{1}{\beta}\right) \frac{\left(V_{p}[k, \ell]\right)_{p=1}^{P}}{\left\|\left(V_{p}^{0}[k, \ell]\right)_{p=1}^{P}\right\|}-\frac{1}{\beta}\left(\lambda_{p}[k, \ell]\right)_{p=1}^{P},
$$

and if $\|x\|=0$ we have

$$
\left(V_{p}^{0}[k, \ell]\right)_{p=1}^{P}=0 .
$$

Hence, $\left(\underline{\underline{\mathfrak{E}}}+1 / \beta \partial \mathrm{A}_{2}\right)^{-1}$ is single-valued. Plugging $\left(\underline{\underline{\underline{E}}}+1 / \beta \mathrm{A}_{2}\right)^{-1}$ into (A.2.11) yields

$$
\underline{W}^{\dagger}=\mathrm{S}_{2}\left(\underline{\mathfrak{C}}_{\underline{B}}(U)-\frac{1}{\beta} \underline{\lambda} ; \frac{1}{\beta}\right)
$$

(ii) Since $\mathcal{J}_{2}$ is a differentiable, convex function in $U$ a necessary and sufficient condition on a minimizer $U^{\dagger}$ of $\mathcal{J}$ is given by $\frac{\partial}{\partial U} \mathcal{J}\left(U^{\dagger}\right)=0$ (see for example Boyd and Vandenberghe [BV04][§4.2]). We obtain

$$
\begin{aligned}
0 & =\mu U^{\dagger}-\mu F+\beta\left(\underline{\mathfrak{C}}_{\underline{B}}^{*} \underline{\underline{\mathfrak{C}}} \underline{\underline{B}}\right)\left(U^{\dagger}\right)-\beta \underline{\mathbb{C}}_{\underline{B}}^{*}(\underline{W})-\underline{\mathfrak{C}}_{\underline{B}}^{*}(\underline{\lambda}) \\
U^{\dagger} & =\mu\left(\mu+\beta\left(\underline{\mathfrak{C}}_{\underline{B}}^{*} \underline{\mathfrak{C}}_{\underline{B}}\right)\right)^{-1}(F)+\beta\left(\mu+\beta\left(\underline{\mathfrak{C}}_{\underline{B}}^{*} \underline{\underline{\mathfrak{C}}} \underline{\underline{B}}\right)\right)^{-1} \underline{\mathfrak{C}}_{\underline{B}}^{*}\left(\underline{W}+\frac{1}{\beta} \underline{\lambda}\right) .
\end{aligned}
$$




\section{A.3 Miscellaneous}

Lemma A.3.1. Let $x_{1}, x_{2}, r \in \mathbb{R}^{m}, y_{1}, y_{2}, s \in \mathbb{R}^{n}$ and $t \in[0,1]$. Define the convex combinations

$$
x_{t}:=t x_{1}+(1-t) x_{2}, \quad y_{t}:=t y_{1}+(1-t) y_{2}, \text { for } t \in[0,1]
$$

If we have

$$
\left\|x_{1}-x_{t}\right\|^{2}+C^{2}\left\|y_{1}-y_{t}\right\|^{2}-\left\|x_{1}-r\right\|^{2}-C^{2}\left\|y_{1}-s\right\|^{2} \geq 0
$$

and

$$
\left\|x_{2}-x_{t}\right\|^{2}+C^{2}\left\|y_{2}-y_{t}\right\|^{2}-\left\|x_{2}-r\right\|^{2}-C^{2}\left\|y_{2}-s\right\|^{2} \geq 0,
$$

for some $C \in \mathbb{R}_{+}$. Then

$$
r=x_{t} \text { and } s=y_{t} \text {. }
$$

Proof. Consider $\mathbb{R}^{n+m}$ then we have by hypothesis

$$
\begin{aligned}
& \left\|\left(x_{1}, C y_{1}\right)-\left(x_{t}, C y_{t}\right)\right\|^{2} \geq\left\|\left(x_{1}, C y_{1}\right)-(r, C s)\right\|^{2}, \\
& \left\|\left(x_{2}, C y_{2}\right)-\left(x_{t}, C y_{t}\right)\right\|^{2} \geq\left\|\left(x_{2}, C y_{2}\right)-(r, C s)\right\|^{2} .
\end{aligned}
$$

By taking the square-root on both sides of (A.3.1) we obtain

$$
\begin{aligned}
& \left\|\left(x_{1}, C y_{1}\right)-\left(x_{t}, C y_{t}\right)\right\| \geq\left\|\left(x_{1}, C y_{1}\right)-(r, C s)\right\|, \\
& \left\|\left(x_{2}, C y_{2}\right)-\left(x_{t}, C y_{t}\right)\right\| \geq\left\|\left(x_{2}, C y_{2}\right)-(r, C s)\right\| .
\end{aligned}
$$

By definition of $x_{t}$ and $y_{t}$ however

$$
\begin{aligned}
\left\|\left(x_{1}, C y_{1}\right)-\left(x_{t}, C y_{t}\right)\right\| & =\left\|(1-t)\left(x_{1}, C y_{1}\right)-(1-t)\left(x_{2}, C y_{2}\right)\right\| \\
& =(1-t)\left\|\left(x_{1}, C y_{1}\right)-\left(x_{2}, C y_{2}\right)\right\|,
\end{aligned}
$$

and

$$
\left\|\left(x_{2}, C y_{2}\right)-\left(x_{t}, C y_{t}\right)\right\|=\left\|-t\left(x_{1}, C y_{1}\right)+t\left(x_{2}, C y_{2}\right)\right\|=t\left\|\left(x_{1}, C y_{1}\right)-\left(x_{2}, C y_{2}\right)\right\| .
$$

We add the previous two equation and compare with (A.3.2)

$$
\left\|\left(x_{1}, C y_{1}\right)-\left(x_{2}, C y_{2}\right)\right\| \geq\left\|\left(x_{1}, C y_{1}\right)-(r, C s)\right\|+\left\|\left(x_{2}, C y_{2}\right)-(r, C s)\right\|,
$$


by triangle inequality we obtain that $(r, s)$ must be a convex combination of $\left(x_{1}, y_{1}\right)$ and $\left(x_{2}, y_{2}\right)$. Hence, there is a $t_{0} \in[0,1]$ such that $r=x_{t_{0}}$ and $s=y_{t_{0}}$. If $t \neq t_{0}$ one of the assumed equalities cannot be true, hence, we obtain

$$
r=x_{t} \text { and } s=y_{t} .
$$




\section{CHAPTER B}

\section{Parameters of the Denoising Experiment}

In this appendix the parameters yielding maximal PSNR computed for Experiment 1 in Chapter 5.1 are reported.

Table B.1

Experiment 1 - Parameters 1/3

\begin{tabular}{|c|c|c|c|c|c|c|c|}
\hline \multirow[t]{2}{*}{ Image } & \multirow[t]{2}{*}{$\sigma$} & \multirow[t]{2}{*}{ Covariance } & \multicolumn{5}{|c|}{ Paramaters } \\
\hline & & & $\mu$ & $y_{1}$ & $y_{2}$ & $r_{1}$ & $r_{2}$ \\
\hline \multirow{12}{*}{ barbara } & 50 & E & 0.02431 & -0.042 & 0.042 & 0.7408 & 1.3499 \\
\hline & 150 & E & 0.00625 & -0.014 & 0.014 & 0.9418 & 1.0618 \\
\hline & 50 & $\mathfrak{C}_{Z_{x}} \mathfrak{C}_{Z_{x}}^{*}$ & 0.0818 & -0.07 & 0.042 & 0.7408 & 1.3499 \\
\hline & 150 & $\mathfrak{C}_{Z_{x}} \mathfrak{C}_{Z_{x}}^{*}$ & 0.00988 & -0.07 & 0.014 & 0.7408 & 1.3499 \\
\hline & 250 & $\mathfrak{C}_{Z_{x}} \mathfrak{C}_{Z_{x}}^{*}$ & 0.00531 & -0.07 & 0.014 & 0.7408 & 1.3499 \\
\hline & 50 & $\mathfrak{C}_{Z_{y}} \mathfrak{C}_{Z_{y}^{*}}^{*}$ & 0.081 & 0.014 & -0.07 & 1.1972 & 0.7408 \\
\hline & 150 & $\mathfrak{C}_{Z_{y}} \mathfrak{C}_{Z_{y}^{*}}^{*}$ & 0.00992 & 0.014 & -0.07 & 1.0618 & 0.9418 \\
\hline & 250 & $\mathfrak{C}_{Z_{y}} \mathfrak{C}_{Z_{y}}^{*}$ & 0.00513 & -0.014 & -0.014 & 0.9418 & 1.0618 \\
\hline & 50 & $\mathfrak{C}_{Z_{y}} \mathfrak{C}_{Z_{x}} \mathfrak{C}_{Z_{x}}^{*} \mathfrak{C}_{Z_{y}}^{*}$ & 0.4515 & -0.07 & 0.014 & 0.7408 & 1.3499 \\
\hline & 150 & $\mathfrak{C}_{Z_{y}} \mathfrak{C}_{Z_{x}} \mathfrak{C}_{Z_{x}^{*}} \mathfrak{C}_{Z_{y}}^{*}$ & 0.015 & 0.014 & -0.07 & 0.8353 & 1.1972 \\
\hline & 100 & $\mathfrak{C}_{Z_{d}} \mathfrak{C}_{Z_{d}}^{*}$ & 0.0218 & -0.07 & 0.014 & 0.7408 & 1.3499 \\
\hline & 250 & $\mathfrak{C}_{Z_{d}} \mathfrak{C}_{Z_{d}}^{*}$ & 0.00581 & -0.07 & 0.014 & 0.7408 & 1.3499 \\
\hline \multirow{12}{*}{ cameraman } & 50 & E & 0.0232 & -0.014 & 0.042 & 0.8353 & 1.1972 \\
\hline & 150 & E & 0.0064 & -0.042 & 0.07 & 0.8353 & 1.1972 \\
\hline & 50 & $\mathfrak{C}_{Z_{x}} \mathfrak{C}_{Z_{x}}^{*}$ & 0.054 & -0.07 & 0.014 & 0.7408 & 1.3499 \\
\hline & 150 & $\mathfrak{C}_{Z_{x}} \mathfrak{C}_{Z_{x}^{*}}^{*}$ & 0.0107 & -0.07 & 0.042 & 0.7408 & 1.3499 \\
\hline & 250 & $\mathfrak{C}_{Z_{x}} \mathfrak{C}_{Z_{x}}^{*}$ & 0.00536 & -0.07 & 0.042 & 0.7408 & 1.1972 \\
\hline & 50 & $\mathfrak{C}_{Z_{y}} \mathfrak{C}_{Z_{y}^{*}}^{*}$ & 0.0543 & 0.014 & -0.07 & 1.1972 & 0.7408 \\
\hline & 150 & $\mathfrak{C}_{Z_{y}} \mathfrak{C}_{Z_{y}}^{*}$ & 0.01078 & 0.014 & -0.07 & 1.0618 & 0.9418 \\
\hline & 250 & $\mathfrak{C}_{Z_{y}} \mathfrak{C}_{Z_{y}}^{*}$ & 0.0054 & 0.014 & -0.07 & 1.0618 & 0.9418 \\
\hline & 50 & $\mathfrak{C}_{Z_{y}} \mathfrak{C}_{Z_{x}} \mathfrak{C}_{Z_{x}^{*}} \mathfrak{C}_{Z_{y}}^{*}$ & 0.199 & 0.014 & -0.07 & 1.0618 & 0.9418 \\
\hline & 150 & $\mathfrak{C}_{Z_{y}} \mathfrak{C}_{Z_{x}} \mathfrak{C}_{Z_{x}^{*}} \mathfrak{C}_{Z_{y}}^{*}$ & 0.0183 & -0.07 & 0.014 & 0.8353 & 1.3499 \\
\hline & 100 & $\mathfrak{c}_{Z_{d}} \mathfrak{C}_{Z_{d}}^{*}$ & 0.0236 & -0.07 & 0.042 & 0.7408 & 1.3499 \\
\hline & 250 & $\mathfrak{C}_{Z_{d}} \mathfrak{C}_{Z_{d}}^{*}$ & 0.00645 & -0.07 & 0.07 & 0.7408 & 1.3499 \\
\hline
\end{tabular}


Table B.2

Experiment 1 - Parameters 2/3

\begin{tabular}{|c|c|c|c|c|c|c|c|}
\hline \multirow[t]{2}{*}{ Image } & \multirow[t]{2}{*}{$\sigma$} & \multirow[t]{2}{*}{ Covariance } & \multicolumn{5}{|c|}{ Paramaters } \\
\hline & & & $\mu$ & $y_{1}$ & $y_{2}$ & $r_{1}$ & $r_{2}$ \\
\hline \multirow{12}{*}{ fish } & 50 & $\mathfrak{E}$ & 0.0276 & -0.014 & 0.014 & 0.9418 & 1.0618 \\
\hline & 150 & $\mathfrak{E}$ & 0.00725 & -0.014 & 0.014 & 0.9418 & 1.0618 \\
\hline & 50 & $\mathfrak{C}_{Z_{x}} \mathfrak{C}_{Z_{x}}^{*}$ & 0.072 & -0.7 & 0.014 & 0.7408 & 1.1972 \\
\hline & 150 & $\mathfrak{C}_{Z_{x}} \mathfrak{C}_{Z_{x}}^{*}$ & 0.0146 & -0.7 & 0.014 & 0.7408 & 1.3499 \\
\hline & 250 & $\mathfrak{C}_{Z_{x}} \mathfrak{C}_{Z_{x}}^{*}$ & 0.0064 & -0.7 & 0.014 & 0.7408 & 1.3499 \\
\hline & 50 & $\mathfrak{C}_{Z_{y}} \mathfrak{C}_{Z_{y}}^{*}$ & 0.0731 & 0.014 & -0.7 & 1.3499 & 0.7408 \\
\hline & 150 & $\mathfrak{C}_{Z_{y}} \mathfrak{C}_{Z_{y}}^{*}$ & 0.0145 & 0.014 & -0.7 & 1.3499 & 0.7408 \\
\hline & 250 & $\mathfrak{c}_{Z_{y}} \mathfrak{C}_{Z_{y}}^{*}$ & 0.00635 & 0.014 & -0.7 & 1.1972 & 0.7408 \\
\hline & 50 & $\mathfrak{C}_{Z_{y}} \mathfrak{C}_{Z_{x}} \mathfrak{C}_{Z_{x}^{*}}^{*} \mathfrak{C}_{Z_{y}}^{*}$ & 0.3115 & -0.7 & 0.014 & 0.8353 & 1.1972 \\
\hline & 150 & $\mathfrak{C}_{Z_{y}} \mathfrak{C}_{Z_{x}} \mathfrak{C}_{Z_{x}}^{*} \mathfrak{C}_{Z_{y}}^{*}$ & 0.034 & -0.7 & 0.014 & 0.8353 & 1.1972 \\
\hline & 100 & $\mathfrak{C}_{Z_{d}} \mathfrak{C}_{Z_{d}}^{*}$ & 0.034 & -0.7 & 0.042 & 0.7408 & 1.3499 \\
\hline & 250 & $\mathfrak{C}_{Z_{d}} \mathfrak{C}_{Z_{d}}^{*}$ & 0.00763 & -0.7 & 0.014 & 0.7408 & 1.3499 \\
\hline \multirow{12}{*}{ boat } & 50 & $\mathfrak{E}$ & 0.0231 & -0.014 & 0.014 & 0.8353 & 1.1972 \\
\hline & 150 & E & 0.00637 & 0.014 & -0.014 & 0.9418 & 1.0618 \\
\hline & 50 & $\mathfrak{C}_{Z_{x}} \mathfrak{C}_{Z_{x}}^{*}$ & 0.051 & -0.07 & 0.014 & 0.7408 & 1.3499 \\
\hline & 150 & $\mathfrak{C}_{Z_{x}}^{*} \mathfrak{C}_{Z_{x}}^{*}$ & 0.0103 & -0.07 & 0.014 & 0.7408 & 1.3499 \\
\hline & 250 & $\mathfrak{C}_{Z_{x}}^{*} \mathfrak{C}_{Z_{x}}^{*}$ & 0.00521 & -0.07 & 0.014 & 0.9418 & 1.0618 \\
\hline & 50 & $\mathfrak{C}_{Z_{y}} \mathfrak{C}_{Z_{y}}^{*}$ & 0.05003 & 0.014 & -0.07 & 1.1972 & 0.07408 \\
\hline & 150 & $\mathfrak{c}_{Z_{y}} \mathfrak{C}_{Z_{y}}^{*}$ & 0.01012 & 0.014 & -0.07 & 1.0618 & 0.9418 \\
\hline & 250 & $\mathfrak{c}_{Z_{y}} \mathfrak{C}_{Z_{y}}^{*}$ & 0.00527 & 0.014 & -0.07 & 1.3499 & 0.7408 \\
\hline & 50 & $\mathfrak{C}_{Z_{y}} \mathfrak{C}_{Z_{x}} \mathfrak{C}_{Z_{x}}^{*} \mathfrak{C}_{Z_{y}}^{*}$ & 0.1715 & -0.07 & 0.014 & 0.7408 & 1.3499 \\
\hline & 150 & $\mathfrak{C}_{Z_{y}} \mathfrak{C}_{Z_{x}} \mathfrak{C}_{Z_{x}^{*}}^{*} \mathfrak{C}_{Z_{y}^{*}}^{*}$ & 0.01622 & -0.07 & 0.014 & 0.8353 & 1.1972 \\
\hline & 100 & $\mathfrak{c}_{Z_{d}} \mathfrak{c}_{Z_{d}}^{*}$ & 0.02195 & -0.07 & 0.042 & 0.7408 & 1.3499 \\
\hline & 250 & $\mathfrak{C}_{Z_{d}} \mathfrak{C}_{Z_{d}}^{*}$ & 0.00583 & -0.07 & 0.042 & 1.0618 & 0.9418 \\
\hline \multirow{12}{*}{ goldhill } & 50 & $\mathfrak{E}$ & 0.02219 & 0.014 & -0.014 & 1.0618 & 0.9418 \\
\hline & 150 & $\mathfrak{E}$ & 0.00615 & -0.014 & 0.014 & 1.0618 & 0.9418 \\
\hline & 50 & $\mathfrak{C}_{Z_{x}} \mathfrak{C}_{Z_{x}}^{*}$ & 0.0458 & -0.07 & 0.014 & 0.7408 & 1.1972 \\
\hline & 150 & $\mathfrak{C}_{Z_{x}} \mathfrak{C}_{Z_{x}}^{*}$ & 0.00969 & -0.07 & 0.014 & 0.7408 & 1.1972 \\
\hline & 250 & $\mathfrak{C}_{Z_{x}} \mathfrak{C}_{Z_{x}}^{*}$ & 0.00502 & -0.07 & 0.014 & 0.9418 & 1.0618 \\
\hline & 50 & $\mathfrak{c}_{Z_{y}} \mathfrak{C}_{Z_{y}}^{*}$ & 0.04618 & 0.014 & -0.07 & 13499 & 0.7408 \\
\hline & 150 & $\mathfrak{C}_{Z_{y}} \mathfrak{C}_{Z_{y}}^{*}$ & 0.00949 & 0.014 & -0.07 & 1.1972 & 0.7408 \\
\hline & 250 & $\mathfrak{C}_{Z_{y}} \mathfrak{C}_{Z_{y}}^{*}$ & 0.005 & 0.014 & -0.07 & 1.1972 & 0.7408 \\
\hline & 50 & $\mathfrak{C}_{Z_{y}} \mathfrak{C}_{Z_{x}} \mathfrak{C}_{Z_{x}}^{*} \mathfrak{C}_{Z_{y}}^{*}$ & 0.1726 & -0.07 & 0.014 & 0.9418 & 1.0618 \\
\hline & 150 & $\mathfrak{C}_{Z_{y}} \mathfrak{C}_{Z_{x}} \mathfrak{C}_{Z_{x}^{*}} \mathfrak{C}_{Z_{y}}^{*}$ & 0.01386 & -0.07 & 0.014 & 0.9418 & 1.0618 \\
\hline & 100 & $\mathfrak{c}_{Z_{d}} \mathfrak{c}_{Z_{d}}^{*}$ & 0.0199 & -0.014 & -0.014 & 1.0618 & 0.9418 \\
\hline & 250 & $\mathfrak{C}_{Z_{d}} \mathfrak{C}_{Z_{d}^{*}}^{*}$ & 0.00563 & 0.042 & -0.07 & 1.0618 & 0.9418 \\
\hline
\end{tabular}


Table B.3

Experiment 1 - Parameters 3/3

\begin{tabular}{|c|c|c|c|c|c|c|c|}
\hline \multirow[t]{2}{*}{ Image } & \multirow[t]{2}{*}{$\sigma$} & \multirow[t]{2}{*}{ Covariance } & \multicolumn{5}{|c|}{ Paramaters } \\
\hline & & & $\mu$ & $y_{1}$ & $y_{2}$ & $r_{1}$ & $r_{2}$ \\
\hline \multirow{12}{*}{ house } & 50 & (E) & 0.020883 & 0.042 & -0.014 & 1.0618 & 0.9418 \\
\hline & 150 & (E) & 0.0064 & 0.014 & 0.014 & 1.0618 & 0.9418 \\
\hline & 50 & $\mathfrak{C}_{Z_{x}} \mathfrak{C}_{Z_{x}}^{*}$ & 0.03867 & -0.07 & 0.014 & 0.8353 & 1.0618 \\
\hline & 150 & $\mathfrak{C}_{Z_{x}} \mathfrak{C}_{Z_{x}}^{*}$ & 0.00961 & -0.07 & 0.014 & 0.9418 & 1.0618 \\
\hline & 150 & $\mathfrak{C}_{Z_{x}} \mathfrak{C}_{Z_{x}}^{*}$ & 0.00506 & -0.07 & 0.014 & 0.9418 & 1.0618 \\
\hline & 50 & $\mathfrak{C}_{Z_{y}} \mathfrak{C}_{Z_{y}^{*}}^{*}$ & 0.03929 & 0.014 & -0.07 & 1.3499 & 0.7408 \\
\hline & 150 & $\mathfrak{C}_{Z_{y}} \mathfrak{C}_{Z_{y}}^{*}$ & 0.00949 & 0.014 & -0.07 & 1.1972 & 0.7408 \\
\hline & 250 & $\mathfrak{C}_{Z_{y}} \mathfrak{C}_{Z_{y}}^{*}$ & 0.00521 & 0.042 & -0.07 & 1.3499 & 0.7408 \\
\hline & 50 & $\mathfrak{C}_{Z_{y}} \mathfrak{C}_{Z_{x}} \mathfrak{C}_{Z_{x}}^{*} \mathfrak{C}_{Z_{y}}^{*}$ & 0.06856 & 0.014 & -0.07 & 1.3499 & 0.7408 \\
\hline & 150 & $\mathfrak{C}_{Z_{y}} \mathfrak{C}_{Z_{x}} \mathfrak{C}_{Z_{x}}^{*} \mathfrak{C}_{Z_{y}}^{*}$ & 0.01416 & 0.014 & -0.07 & 1.0618 & 0.9418 \\
\hline & 100 & $\mathfrak{C}_{Z_{d}} \mathfrak{C}_{Z_{d}}^{*}$ & 0.01919 & 0.014 & -0.014 & 1.0618 & 0.9418 \\
\hline & 250 & $\mathfrak{c}_{Z_{d}} \mathfrak{C}_{Z_{d}}^{*}$ & 0.00603 & 0.042 & -0.07 & 1.1972 & 0.8353 \\
\hline \multirow{12}{*}{ lung } & 50 & E & 0.02195 & 0.014 & -0.014 & 1.0618 & 0.9418 \\
\hline & 150 & $\mathfrak{E}$ & 0.00607 & 0.014 & -0.014 & 1.0618 & 0.9418 \\
\hline & 50 & $\mathfrak{C}_{Z_{x}} \mathfrak{C}_{Z_{x}}^{*}$ & 0.04663 & -0.07 & 0.014 & 0.7408 & 1.1972 \\
\hline & 150 & $\mathfrak{C}_{Z_{x}} \mathfrak{C}_{Z_{x}}^{*}$ & 0.00926 & -0.07 & 0.014 & 0.9418 & 1.0618 \\
\hline & 250 & $\mathfrak{C}_{Z_{x}} \mathfrak{C}_{Z_{x}}^{*}$ & 0.00474 & -0.07 & 0.014 & 1.0618 & 0.9418 \\
\hline & 50 & $\mathfrak{C}_{Z_{y}} \mathfrak{C}_{Z_{y}}^{*}$ & 0.046775 & 0.014 & -0.07 & 1.3499 & 0.7408 \\
\hline & 150 & $\mathfrak{C}_{Z_{y}} \mathfrak{C}_{Z_{y}}^{*}$ & 0.00966 & 0.014 & -0.07 & 1.3499 & 0.7408 \\
\hline & 250 & $\mathfrak{C}_{Z_{y}} \mathfrak{C}_{Z_{y}}^{*}$ & 0.00475 & 0.042 & -0.07 & 1.3499 & 0.7408 \\
\hline & 50 & $\mathfrak{C}_{Z_{y}} \mathfrak{C}_{Z_{x}} \mathfrak{C}_{Z_{x}}^{*} \mathfrak{C}_{Z_{y}}^{*}$ & 0.28297 & 0.014 & -0.07 & 1.3499 & 0.8353 \\
\hline & 150 & $\mathfrak{C}_{Z_{y}} \mathfrak{C}_{Z_{x}} \mathfrak{C}_{Z_{x}}^{*} \mathfrak{C}_{Z_{y}}^{*}$ & 0.01443 & 0.014 & -0.07 & 1.1972 & 0.8353 \\
\hline & 100 & $\mathfrak{C}_{Z_{d}} \mathfrak{C}_{Z_{d}}^{*}$ & 0.02003 & 0.042 & -0.07 & 1.1972 & 0.8353 \\
\hline & 250 & $\mathfrak{c}_{Z_{d}} \mathfrak{C}_{Z_{d}}^{*}$ & 0.00551 & 0.014 & -0.07 & 1.1972 & 0.8353 \\
\hline
\end{tabular}




\section{CHAPTER C}

\section{Construction of the Wavelet-Frame-Based Filters}

In this appendix the exact construction of the biorthogonal and orthogonal Laplacian B-spline filters $A, \underline{B}_{\mathrm{bi}}, \widetilde{\widetilde{B}}_{\mathrm{bi}}, \underline{B}_{\text {orth }}$ and $\underline{\widetilde{B}}_{\text {orth }}$ of Chapter 5.2 is given.

\section{C.1 Isotropic Polyharmonic B-Spline Wavelet Frames}

Let in the following $\gamma \in \mathbb{R}_{+}$and $N, I \in \mathbb{N}$ be fixed, first define the isotropic polyharmonic $B$-Spline $f_{\gamma}: \mathbb{R}^{2} \rightarrow \mathbb{R}$ as detailed in Van de Ville et al. [VDVBU05].

$$
f_{\gamma}(x, y):=\left(\frac{4\left(\sin ^{2}\left(\frac{x}{2}\right)+\sin ^{2}\left(\frac{y}{2}\right)\right)-\frac{8}{3}\left(\sin \left(\frac{x}{2}\right) \sin \left(\frac{y}{2}\right)\right)}{\left(x^{2}+y^{2}\right)}\right)^{\frac{\gamma}{2}} .
$$

Define the autocorrelation function $a_{\gamma}: \mathbb{R}^{2} \rightarrow \mathbb{R}$ of the function $f_{\gamma}$ as

$$
a_{\gamma}(x, y):=\sum_{r, s \in \mathbb{Z}}\left(f_{\gamma}(x+2 \pi r, y+2 \pi s)^{2} .\right.
$$

To construct the input filter recall the convention 5.0.6, restated below for convenience.

Convention C.1.1. For an entry $[k, \ell]$, with $0 \leq k \leq n-1$ and $0 \leq \ell \leq m-1$, set $\omega_{k}, \omega_{\ell} \in[-\pi, \pi)$ as

$$
\omega_{k}:=\left\{\begin{array}{ll}
\frac{2 \pi k}{n} & \text { if } k<\frac{n}{2} \\
-2 \pi+\frac{2 \pi k}{n} & \text { else }
\end{array} \quad \text { and } \quad \omega_{\ell}:=\left\{\begin{array}{ll}
\frac{2 \pi \ell}{m} & \text { if } \ell<\frac{m}{2} \\
-2 \pi+\frac{2 \pi \ell}{m} & \text { else }
\end{array} .\right.\right.
$$

For the biorthogonal, as well as, the orthogonal input filters define

$$
\widehat{A}[k, \ell]:=\left\|\frac{f_{\gamma}\left(2^{I} \omega_{k}, 2^{I} \omega_{\ell}\right)}{\sqrt{a_{\gamma}\left(2^{I} \omega_{k}, 2^{I} \omega_{\ell}\right)}}\right\|^{2} .
$$

Let now $p \in\{0,1, \ldots, I-1, I\}$ be a scale, we will compute wavelet functions via dyadic sub-sampling [Mal98]. To simplify notations we first introduce the refinement filter 
function $h_{\gamma}: \mathbb{R}^{2} \rightarrow \mathbb{R}$ by

$$
h_{\gamma}(x, y):=\frac{2 f_{\gamma}(-2 x,-2 y)}{f_{\gamma}(-x,-y)}
$$

see [VDVBU05][Eq.(29)]. Let us compute the primal wavelet frames $T_{p, s}^{\text {primal }} \in \mathbb{R}^{n \times m}$, for $s=1,2,3$.

$$
\begin{aligned}
T_{p, 1}^{\text {primal }}[k, \ell]= & \frac{1}{2} \exp \left(-i\left(2^{p-1} \omega_{k}+\pi\right)\right) h_{\gamma}\left(2^{p-1} \omega_{k}+\pi, 2^{p-1} \omega_{\ell}\right) \\
& \times a_{\gamma}\left(2^{p-1} \omega_{k}+\pi, 2^{p-1} \omega_{\ell}\right) f_{\gamma}\left(2^{p-1} \omega_{k}, 2^{p-1} \omega_{\ell}\right), \\
T_{p, 2}^{\text {primal }}[k, \ell]= & \frac{1}{2} \exp \left(-i\left(2^{p-1} \omega_{k}+\pi\right)\right) h_{\gamma}\left(2^{p-1} \omega_{k}, 2^{p-1} \omega_{\ell}+\pi\right) \\
& \times a_{\gamma}\left(2^{p-1} \omega_{k}, 2^{p-1} \omega_{\ell}+\pi\right) f_{\gamma}\left(2^{p-1} \omega_{k}, 2^{p-1} \omega_{\ell}\right), \\
T_{p, 3}^{\text {primal }}[k, \ell]= & \frac{1}{2} \exp \left(-i\left(2^{p-1} \omega_{k}+\pi\right)\right) h_{\gamma}\left(2^{p-1} \omega_{k}+\pi, 2^{p-1} \omega_{\ell}+\pi\right) \\
& \times a_{\gamma}\left(2^{p-1} \omega_{k}+\pi, 2^{p-1} \omega_{\ell}+\pi\right) f_{\gamma}\left(2^{p-1} \omega_{k}, 2^{p-1} \omega_{\ell}\right) .
\end{aligned}
$$

Their dual counterparts are constructed using

$$
\begin{aligned}
T_{p, 1}^{\text {dual }}[k, \ell]= & \frac{1}{2} \exp \left(-i\left(2^{p-1} \omega_{k}+\pi\right)\right) \frac{h_{\gamma}\left(2^{p-1} \omega_{k}+\pi, 2^{p-1} \omega_{\ell}\right)}{a_{\gamma}\left(2^{p} \omega_{k}, 2^{p} \omega_{\ell}\right)} \\
& \times \frac{f_{\gamma}\left(2^{p-1} \omega_{k}, 2^{p-1} \omega_{\ell}\right)}{a_{\gamma}\left(2^{p-1} \omega_{k}, 2^{p-1} \omega_{\ell}\right)}, \\
T_{p, 2}^{\text {dual }}[k, \ell]= & \frac{1}{2} \exp \left(-i\left(2^{p-1} \omega_{k}+\pi\right)\right) \frac{h_{\gamma}\left(2^{p-1} \omega_{k}, 2^{p-1} \omega_{\ell}+\pi\right)}{a_{\gamma}\left(2^{p} \omega_{k}, 2^{p} \omega_{\ell}\right)} \\
& \times \frac{f_{\gamma}\left(2^{p-1} \omega_{k}, 2^{p-1} \omega_{\ell}\right)}{a_{\gamma}\left(2^{p-1} \omega_{k}, 2^{p-1} \omega_{\ell}\right)}, \\
T_{p, 3}^{\text {dual }}[k, \ell]= & \frac{1}{2} \exp \left(-i\left(2^{p-1} \omega_{k}+\pi\right)\right) \frac{h_{\gamma}\left(2^{p-1} \omega_{k}+\pi, 2^{p-1} \omega_{\ell}+\pi\right)}{a_{\gamma}\left(2^{p} \omega_{k}, 2^{p} \omega_{\ell}\right)} \\
& \times \frac{f_{\gamma}\left(2^{p-1} \omega_{k}, 2^{p-1} \omega_{\ell}\right)}{a_{\gamma}\left(2^{p-1} \omega_{k}, 2^{p-1} \omega_{\ell}\right)} .
\end{aligned}
$$


Finally, to obtain orthogonal wavelet frames we compute

$$
\begin{aligned}
T_{p, 1}^{\mathrm{orth}}[k, \ell]= & \frac{1}{2} \exp \left(-i\left(2^{p-1} \omega_{k}+\pi\right)\right) \frac{h_{\gamma}\left(2^{p-1} \omega_{k}+\pi, 2^{p-1} \omega_{\ell}\right)}{\sqrt{a_{\gamma}\left(2^{p} \omega_{k}, 2^{p} \omega_{\ell}\right)}} \\
& \times \sqrt{a_{\gamma}\left(2^{p-1} \omega_{k}+\pi, 2^{p-1} \omega_{\ell}\right)} \frac{f_{\gamma}\left(2^{p-1} \omega_{k}, 2^{p-1} \omega_{\ell}\right)}{\sqrt{a_{\gamma}\left(2^{p-1} \omega_{k}, 2^{p-1} \omega_{\ell}\right)}}, \\
T_{p, 2}^{\text {orth }}[k, \ell]= & \frac{1}{2} \exp \left(-i\left(2^{p-1} \omega_{k}+\pi\right)\right) \frac{h_{\gamma}\left(2^{p-1} \omega_{k}, 2^{p-1} \omega_{\ell}+\pi\right)}{\sqrt{a_{\gamma}\left(2^{p} \omega_{k}, 2^{p} \omega_{\ell}\right)}} \\
& \times \sqrt{a_{\gamma}\left(2^{p-1} \omega_{k}, 2^{p-1} \omega_{\ell}+\pi\right)} \frac{f_{\gamma}\left(2^{p-1} \omega_{k}, 2^{p-1} \omega_{\ell}\right)}{\sqrt{a_{\gamma}\left(2^{p-1} \omega_{k}, 2^{p-1} \omega_{\ell}\right)}}, \\
T_{p, 3}^{\text {orth }}[k, \ell]= & \frac{1}{2} \exp \left(-i\left(2^{p-1} \omega_{k}+\pi\right)\right) \frac{h_{\gamma}\left(2^{p-1} \omega_{k}+\pi, 2^{p-1} \omega_{\ell}+\pi\right)}{\sqrt{a_{\gamma}\left(2^{p} \omega_{k}, 2^{p} \omega_{\ell}\right)}} \\
& \times \sqrt{a_{\gamma}\left(2^{p-1} \omega_{k}+\pi, 2^{p-1} \omega_{\ell}+\pi\right)} \frac{f_{\gamma}\left(2^{p-1} \omega_{k}, 2^{p-1} \omega_{\ell}\right)}{\sqrt{a_{\gamma}\left(2^{p-1} \omega_{k}, 2^{p-1} \omega_{\ell}\right)}},
\end{aligned}
$$

\section{C.2 Directionality via the Riesz Transform}

To obtain the final filters $\underline{B}$ and $\underline{\widetilde{B}}$ decompose them into several directions by the $N$-th order Riesz transform. For more on the Riesz transform in signal processing see Unser and Van de Ville [UVDV10], and for its application to the wavelet frames constructed via the isotropic polyharmonic B-spline see Unser et al. [USVDV09].

Define for this purpose for $n=1,2, \ldots, N$ the matrices $R_{n} \in \mathbb{R}^{n \times m}$ by

$$
R_{n}[k, \ell]:=(-i)^{N} \sqrt{\frac{N !}{n !(N-n) !}} \frac{\omega_{k}^{n} \omega_{\ell}^{N-n}}{\left(\omega_{k}^{2}+\omega_{\ell}^{2}\right)^{\frac{N}{2}}},
$$

see [UVDV10][Eq.(10) and (4)]. Now we are ready to define $\underline{B}$ and $\underline{\widetilde{B}}$. Bi-Orthogonal Case: For $p=0, \ldots, I, n=1, \ldots, N, s=1,2,3$ define

$$
\begin{aligned}
& \widehat{B}_{p, n, s}[k, \ell]=T_{p, s}^{\text {primal }}[k, \ell] R_{n}[k, \ell] \\
& \widehat{\widetilde{B}}_{p, n, s}[k, \ell]=T_{p, s}^{\text {dual }}[k, \ell] R_{n}[k, \ell] .
\end{aligned}
$$

Orthogonal Case: For $p=0, \ldots, I, n=1, \ldots, N, s=1,2,3$ define

$$
\widehat{B}_{p, n, s}[k, \ell]=\widetilde{B}_{p, n, s}[k, \ell]=T_{p, s}^{\text {ortho }}[k, \ell] R_{n}[k, \ell] .
$$




\section{C.3 Numerical Adjustments}

First, note that to compute $\underline{B}$ and $\underline{\widetilde{B}}$ in the bi-orthogonal and orthogonal case we have to evaluate the infinite sum in (C.1.2). In all computations the following approximation

$$
a_{\gamma}(x, y):=\sum_{r, s \in\{-10, \ldots, 10\}}\left(f_{\gamma}(x+2 \pi r, y+2 \pi s)^{2},\right.
$$

is used. The resulting input filter do not satisfying the (NEPC), to scale them down

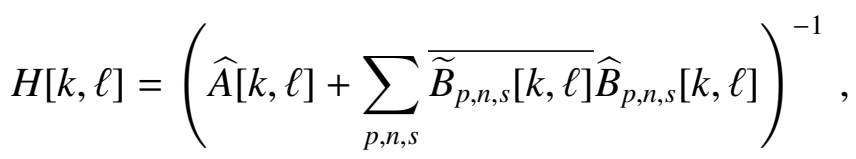

is computed and multiplied to all Fourier transformed filters (including $A$ ) entry-wise.

Secondly, the resulting entries of the filters, when transferred back to the spacial domain, have non-zero imaginary parts. Partly this is due to numerical error, partly due to the correction by $H$. To deal with this, the imaginary part is cut off. A consequence of this adjustment is that for all filters used in Chapter 5.2 the (CPC) is satisfied. 


\section{CHAPTER D}

\section{Additional Results for SNoQE}

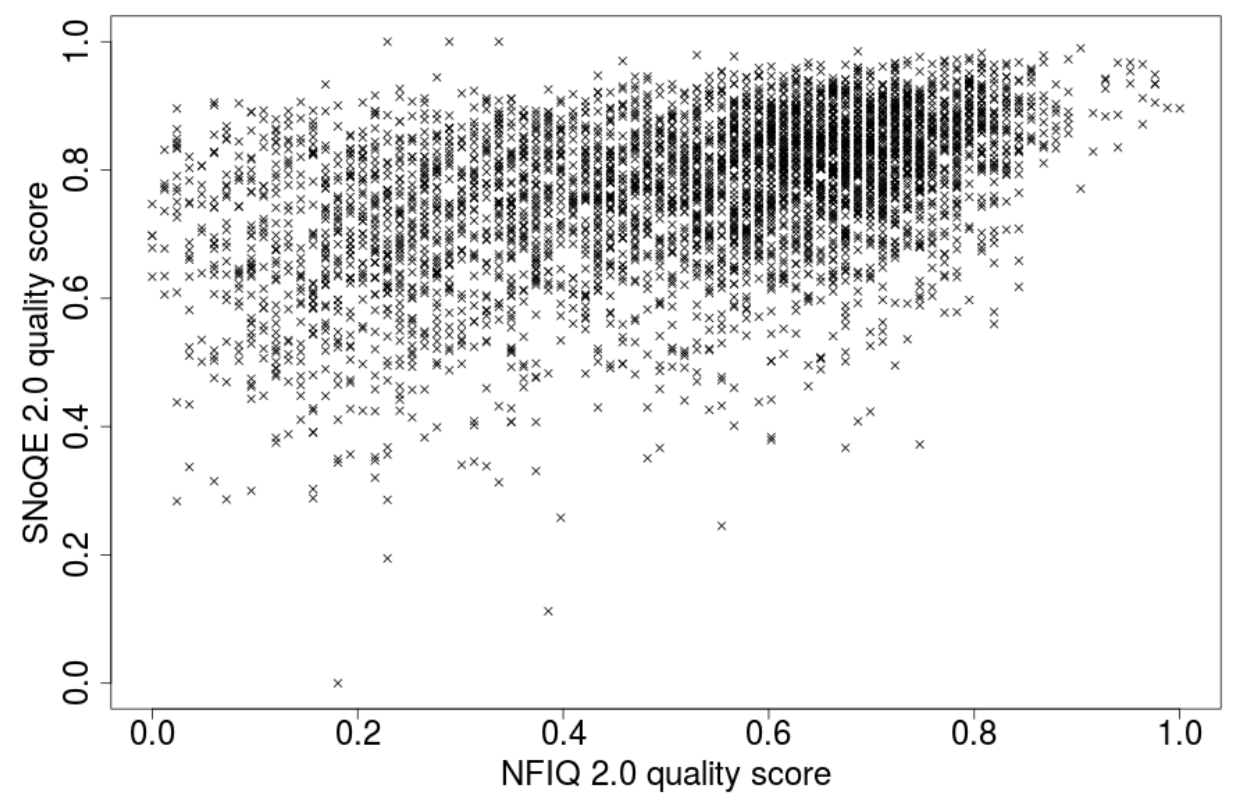

Figure D.1: Scatter plot of NFIQ 2.0 values versus SNoQE values on the NIST SD4 after histogram spreading. 
ERC of DNoQE under bozorth3

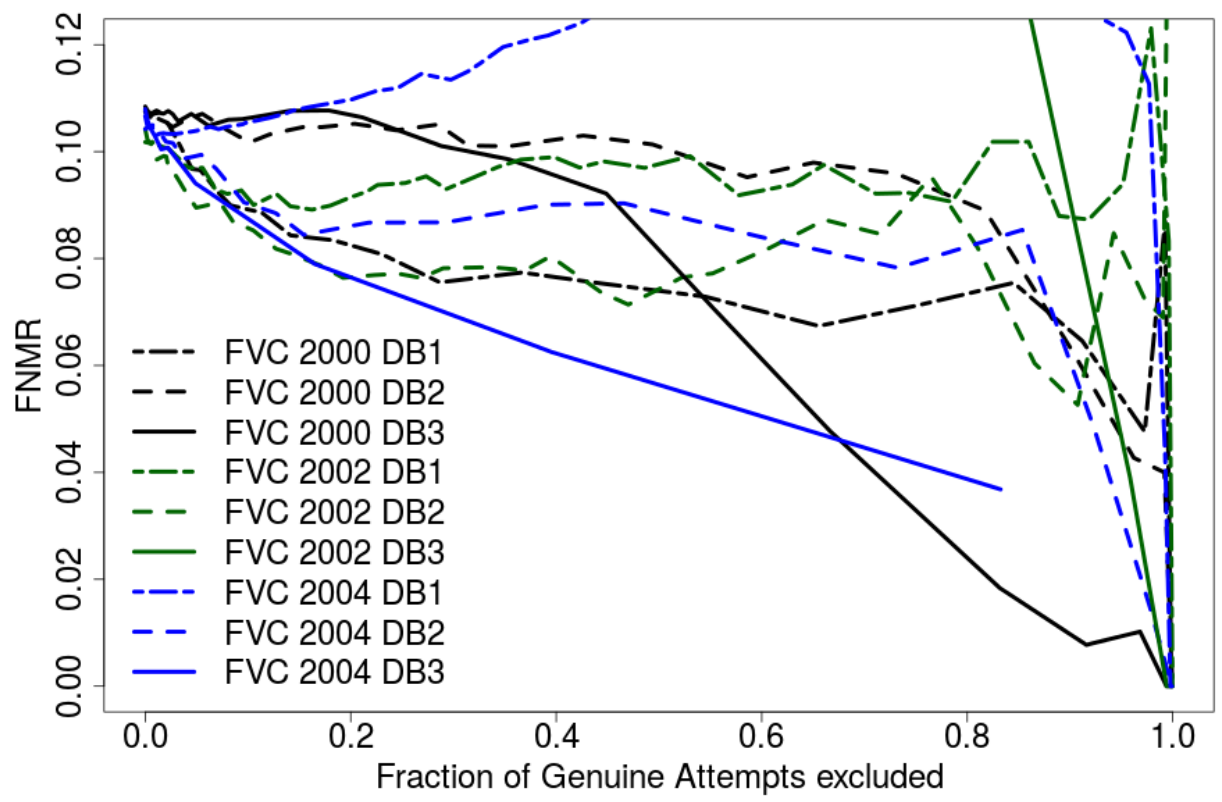

Figure D.2: ERC curves for DNoQE with bozorth3 of all FVC databases.

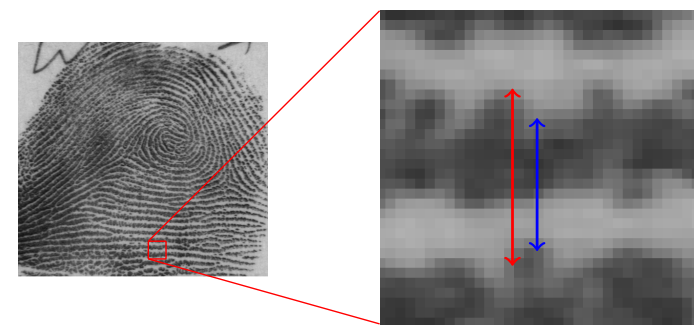

Figure D.3: Heuristic choice of $R_{95}=17$ and $R_{\mathrm{Med}}=14$ underlying $r$ and $\alpha$ in (III.1) and (III.2) for the NIST SD4: The red arrow is 17 pixels long, while the blue arrow is only 13 pixel long. 


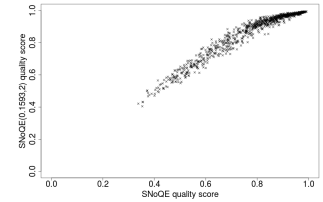

(a)

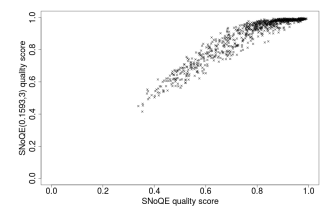

(e)

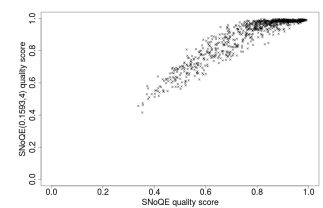

(i)

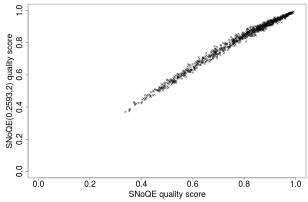

(b)

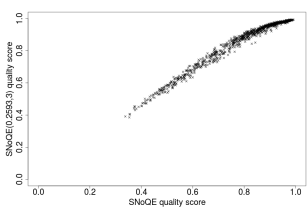

(f)

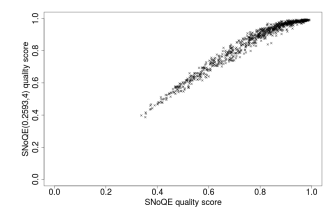

(j)

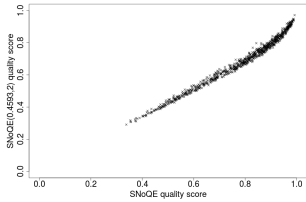

(c)

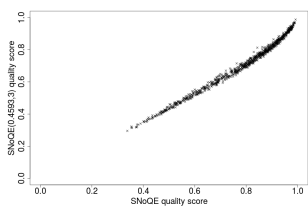

(g)

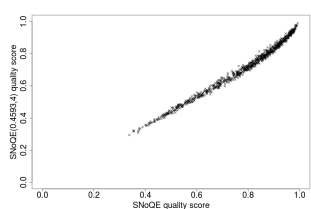

(k)

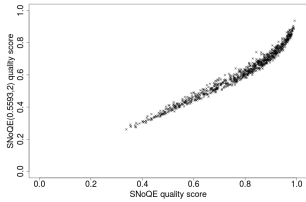

(d)

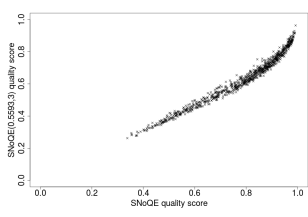

(h)

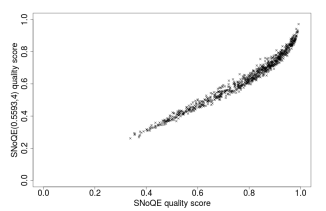

(1)

Figure D.4: Scatter Plots of the SNoQE values of the FVC 2002 DB3 (capacitive scanner) calculated with parameter choices from Tab. III versus their SNoQE values with variable $r$ and $\alpha$ (First column: $\alpha=0.1593$, second column: $\alpha=0.2593$, third column: $\alpha=0.4593$ and fourth column: $\alpha=0.5593$; First row: $r=2$, second row: $r=3$ and third row: $r=4)$. 


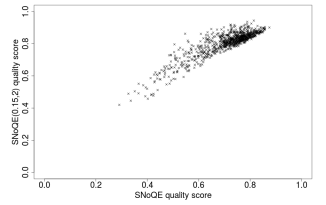

(a)

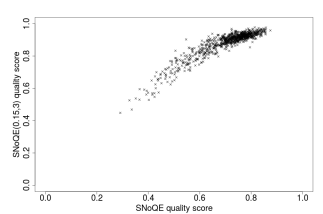

(e)

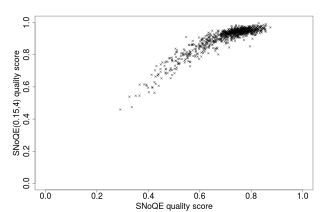

(i)

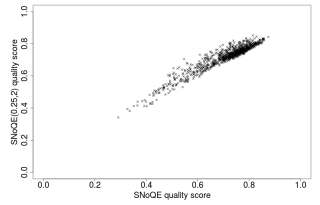

(b)

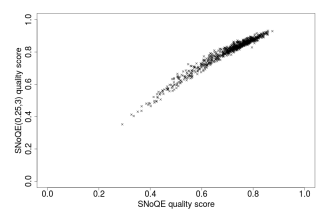

(f)

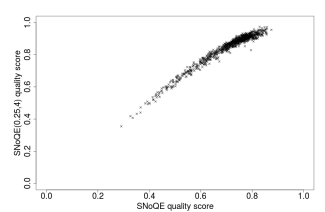

(j)

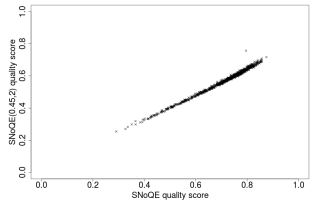

(c)

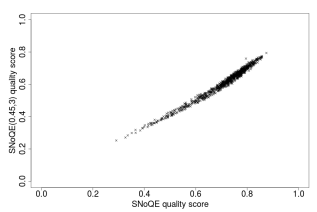

(g)

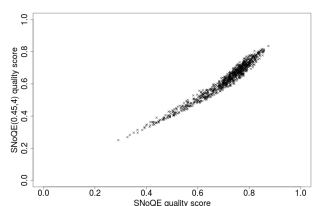

(k)

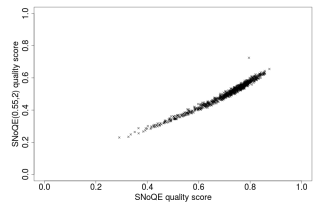

(d)

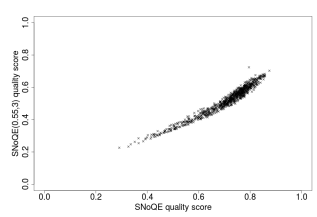

(h)

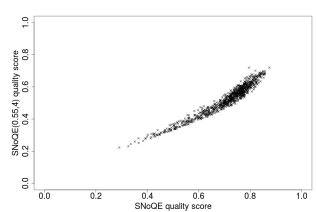

(1)

Figure D.5: Scatter Plots of the SNoQE values of the FVC 2004 DB3 (thermal scanner) calculated with parameter choices from Tab. III versus their SNoQE values with variable $r$ and $\alpha$ (First column: $\alpha=0.15$, second column: $\alpha=0.25$, third column: $\alpha=0.45$ and fourth column: $\alpha=0.55$; First row: $r=2$, second row: $r=3$ and third row: $r=4$ ). 


\section{Bibliography}

[AABFC05] J.-F. Aujol, G. Aubert, L. Blanc-Féraud, and A. Chambolle. Image decomposition into a bounded variation component and an oscillating component. J. Math. Imaging Vision, 22(1):71-88, 2005.

[ABCH08] A. Almansa, C. Ballester, V. Caselles, and G. Haro. A TV based restoration model with local constraints. J. Sci. Comput., 34(3):209-236, 2008.

[AC05] J.-F. Aujol and A. Chambolle. Dual Norms and Image Decomposition Models. International Journal of Computer Vision, 63(1):85-104, 2005.

[ACHR06] A. Almansa, V. Caselles, G. Haro, and B. Rougé. Restoration and zoom of irregularly sampled, blurred, and noisy images by accurate total variation minimization with local constraints. Multiscale Model. Simul., 5(1):235-272, 2006.

[AE84] J.-P. Aubin and I. Ekeland. Applied nonlinear analysis. Pure and Applied Mathematics (New York). John Wiley \& Sons, Inc., New York, 1984. A Wiley-Interscience Publication.

[AFFOG ${ }^{+}$07] F. Alonso-Fernandez, J. Fierrez, J. Ortega-Garcia, J. GonzalezRodriguez, H. Fronthaler, K. Kollreider, and J. Bigun. A Comparative Study of Fingerprint Image-Quality Estimation Methods. IEEE Transactions on Information Forensics and Security, 2(4):734 - 743, 2007.

[AFRM ${ }^{+}$08] F. Alonso-Fernandez, F. Roli, G. Marcialis, J. Fierrez, J. Ortega-Garcia, and J. Gonzalez-Rodriguez. Performance of fingerprint quality measures depending on sensor technology. Journal of Electronic Imaging, 17(1):011008, January 2008.

[AG06] J.-F. Aujol and G. Gilboa. Constrained and SNR-based solutions for TV-Hilbert space image denoising. J. Math. Imaging Vision, 26(1-2):217237, 2006. 
[AGCO06] J.-F. Aujol, G. Gilboa, T. Chan, and S. Osher. Structure-Texture Image Decomposition-Modeling, Algorithms, and Parameter Selection. International Journal of Computer Vision, 67(1):111-136, 2006.

[AK06] G. Aubert and P. Kornprobst. Mathematical problems in image processing, volume 147 of Applied Mathematical Sciences. Springer, New York, second edition, 2006. Partial differential equations and the calculus of variations, With a foreword by Olivier Faugeras.

[AMP04] L. Ambrosio, M. Miranda, Jr., and D. Pallara. Special functions of bounded variation in doubling metric measure spaces. In Calculus of variations: topics from the mathematical heritage of E. De Giorgi, volume 14 of Quad. Mat., pages 1-45. Dept. Math., Seconda Univ. Napoli, Caserta, 2004.

[Auj09] J.-F. Aujol. Some first-order algorithms for total variation based image restoration. J. Math. Imaging Vision, 34(3):307-327, 2009.

[BB97] Y. Bobichon and A. Bijaoui. Regularized multiresolution methods for astronomical image enhancement. 3169:36-46, 1997.

$\left[\mathrm{BBD}^{+} 06\right]$ B. Berkels, M. Burger, M. Droske, O. Nemitz, and M. Rumpf. Cartoon Extraction Based on Anisotropic Image Classification Vision, Modeling, and Visualization. In Vision, Modeling, and Visualization Proceedings, 2006.

[BC11] H. H. Bauschke and P. L. Combettes. Convex analysis and monotone operator theory in Hilbert spaces. CMS Books in Mathematics/Ouvrages de Mathématiques de la SMC. Springer, New York, 2011. With a foreword by Hédy Attouch.

[BCM05] A. Buades, B. Coll, and J. M. Morel. A review of image denoising algorithms, with a new one. Multiscale Model. Simul., 4(2):490-530, 2005.

[BCRS03] M. Bertalmio, V. Caselles, B. Rougé, and A. Solé. TV based image restoration with local constraints. J. Sci. Comput., 19(1-3):95-122, 2003. Special issue in honor of the sixtieth birthday of Stanley Osher.

[BDH13] K. Bredies, Y. Dong, and M. Hintermüller. Spatially dependent regularization parameter selection in total generalized variation models for image restoration. Int. J. Comput. Math., 90(1):109-123, 2013. 
[Ber82] D. P. Bertsekas. Constrained optimization and Lagrange multiplier methods. Computer Science and Applied Mathematics. Academic Press, Inc. [Harcourt Brace Jovanovich, Publishers], New York-London, 1982.

[BKP10] K. Bredies, K. Kunisch, and T. Pock. Total generalized variation. SIAM J. Imaging Sci., 3(3):492-526, 2010.

[BL16a] A. Buades and J.-L. Lisani. Directional Filters for Cartoon + Texture Image Decomposition. Image Processing On Line, 6:75-88, 2016.

[BL16b] A. Buades and J. L. Lisani. Directional filters for color cartoon+texture image and video decomposition. J. Math. Imaging Vision, 55(1):125-135, 2016.

[BLMV10] A. Buades, T. Le, J.-M. Morel, and L. Vese. Fast cartoon + texture image filters. IEEE Trans. Image Process., 19(8):1978-1986, 2010.

[BLMV11] A. Buades, T. Le, J.-M. Morel, and L. Vese. Cartoon+Texture Image Decomposition. Image Processing On Line, 1:200-207, 2011.

[Bou89] N. Bourbaki. Algebra. I. Chapters 1-3. Elements of Mathematics (Berlin). Springer-Verlag, Berlin, 1989. Translated from the French, Reprint of the 1974 edition.

[Bov09] A. C. Bovik. The essential guide to image processing. Academic Press, 2009.

[BP10] M. Bergounioux and L. Piffet. A second-order model for image denoising. Set-Valued Var. Anal., 18(3-4):277-306, 2010.

[Bre14] K. Bredies. Recovering piecewise smooth multichannel images by minimization of convex functionals with total generalized variation penalty. In Efficient algorithms for global optimization methods in computer vision, pages 44-77. Springer, 2014.

[BT09] A. Beck and M. Teboulle. A fast iterative shrinkage-thresholding algorithm for linear inverse problems. SIAM J. Imaging Sci., 2(1):183-202, 2009.

[BV04] S. Boyd and L. Vandenberghe. Convex optimization. Cambridge University Press, Cambridge, 2004. 
[CCN15] V. Caselles, A. Chambolle, and M. Novaga. Total variation in imaging. In Handbook of mathematical methods in imaging. Vol. 1, 2, 3, pages 1455-1499. Springer, New York, 2015.

[CDDY06] E. Candès, L. Demanet, D. Donoho, and L. Ying. Fast discrete curvelet transforms. Multiscale Model. Simul., 5(3):861-899, 2006.

[CDOS12] J.-F. Cai, B. Dong, S. Osher, and Z. Shen. Image restoration: total variation, wavelet frames, and beyond. J. Amer. Math. Soc., 25(4):10331089, 2012.

[CE05] T. F. Chan and S. Esedoglu. Aspects of total variation regularized $L^{1}$ function approximation. SIAM J. Appl. Math., 65(5):1817-1837, 2005.

[Cel69] A. Cellina. Approximation of set valued functions and fixed point theorems. Ann. Mat. Pura Appl. (4), 82:17-24, 1969.

[CGP09] A. Caboussat, R. Glowinski, and V. Pons. An augmented Lagrangian approach to the numerical solution of a non-smooth eigenvalue problem. J. Numer. Math., 17(1):3-26, 2009.

[Cha04] A. Chambolle. An algorithm for total variation minimization and applications. J. Math. Imaging Vision, 20(1-2):89-97, 2004. Special issue on mathematics and image analysis.

[Chu92] C. K. Chui. An introduction to wavelets, volume 1 of Wavelet Analysis and its Applications. Academic Press, Inc., Boston, MA, 1992.

[CJY04] T. Chen, X. Jiang, and W. Yau. Fingerprint Image Quality Analysis. In Proc. Int. Conf. on Image Processing, volume 2, pages 1253-1256, 2004.

[CL97] A. Chambolle and P.-L. Lions. Image recovery via total variation minimization and related problems. Numerische Mathematik, 76(2):167-188, 1997.

[CLMC92] F. Catté, P.-L. Lions, J.-M. Morel, and T. Coll. Image selective smoothing and edge detection by nonlinear diffusion. SIAM J. Numer. Anal., 29(1):182-193, 1992.

[CMM00] T. Chan, A. Marquina, and P. Mulet. High-order total variation-based image restoration. SIAM J. Sci. Comput., 22(2):503-516, 2000. 
[CP11] A. Chambolle and T. Pock. A first-order primal-dual algorithm for convex problems with applications to imaging. J. Math. Imaging Vision, 40(1):120-145, 2011.

[CP16] A. Chambolle and T. Pock. An introduction to continuous optimization for imaging. Acta Numer., 25:161-319, 2016.

[CP17] Y. Chen and T. Pock. Trainable nonlinear reaction diffusion: A flexible framework for fast and effective image restoration. IEEE transactions on pattern analysis and machine intelligence, 39(6):1256-1272, 2017.

[CvGO11] R. Choksi, Y. van Gennip, and A. Oberman. Anisotropic total variation regularized $L^{1}$ approximation and denoising/deblurring of $2 \mathrm{D}$ bar codes. Inverse Probl. Imaging, 5(3):591-617, 2011.

[CW98] T. F. Chan and C.-K. Wong. Total variation blind deconvolution. IEEE transactions on Image Processing, 7(3):370-375, 1998.

[CYV00a] S. G. Chang, B. Yu, and M. Vetterli. Adaptive wavelet thresholding for image denoising and compression. IEEE Trans. Image Process., 9(9):1532-1546, 2000.

[CYV00b] S. G. Chang, B. Yu, and M. Vetterli. Spatially adaptive wavelet thresholding with context modeling for image denoising. IEEE Trans. Image Process., 9(9):1522-1531, 2000.

[DGA88] E. De Giorgi and L. Ambrosio. Un nuovo tipo di funzionale del calcolo delle variazioni. Atti della Accademia Nazionale dei Lincei. Classe di Scienze Fisiche, Matematiche e Naturali. Rendiconti Lincei. Matematica e Applicazioni, 82(2):199-210, 1988.

[DGB07] W. Dubitzky, M. Granzow, and D. Berrar. Fundamentals of Data Mining in Genomics and Proteomics. Springer Science, 2007.

[DHRC11] Y. Dong, M. Hintermüller, and M. M. Rincon-Camacho. Automated regularization parameter selection in multi-scale total variation models for image restoration. J. Math. Imaging Vision, 40(1):82-104, 2011.

[DJ94] D. Donoho and I. Johnstone. Ideal spatial adaptation by wavelet shrinkage. Biometrika, 81(3):425-455, 1994.

[DJ95] D. L. Donoho and I. M. Johnstone. Adapting to unknown smoothness via wavelet shrinkage. J. Amer. Statist. Assoc., 90(432):1200-1224, 1995. 
[DMFLM09] G. Dal Maso, I. Fonseca, G. Leoni, and M. Morini. A higher order model for image restoration: the one-dimensional case. SIAM J. Math. Anal., 40(6):2351-2391, 2009.

[DS06a] J. Darbon and M. Sigelle. Image restoration with discrete constrained total variation. I. Fast and exact optimization. J. Math. Imaging Vision, 26(3):261-276, 2006.

[DS06b] J. Darbon and M. Sigelle. Image restoration with discrete constrained total variation. II. Levelable functions, convex priors and non-convex cases. J. Math. Imaging Vision, 26(3):277-291, 2006.

[DS15] Y. A. Ding and I. Selesnick. Artifact-Free Wavelet Denoising: Nonconvex Sparse Regularization, Convex Optimization. IEEE Signal Processing Letters, 22:1364-1368, 092015.

[EB92] J. Eckstein and D. P. Bertsekas. On the Douglas-Rachford splitting method and the proximal point algorithm for maximal monotone operators. Math. Programming, 55(3, Ser. A):293-318, 1992.

[ET99] I. Ekeland and R. Témam. Convex analysis and variational problems, volume 28 of Classics in Applied Mathematics. Society for Industrial and Applied Mathematics (SIAM), Philadelphia, PA, english edition, 1999. Translated from the French.

[FAAC09] G. Facciolo, A. Almansa, J.-F. Aujol, and V. Caselles. Irregular to regular sampling, denoising, and deconvolution. Multiscale Model. Simul., 7(4):1574-1608, 2009.

[FACOGJ06] J. Fierrez-Aguilar, Y. Chen, J. Ortega-Garcia, and A. Jain. Incorporating Image Quality in Multi-algorithm Fingerprint Verification. In Proc. ICB, pages 213-220, Hong Kong, China, January 2006.

[FKB06] H. Fronthaler, K. Kollreider, and J. Bigun. Automatic Image Quality Assessment with Application in Biometrics. In In IEEE Workshop on Biometrics, in association with CVPR-06, pages 30-35, 2006.

[Gab83] D. Gabay. Applications of the method of multipliers to variational inequalities. In Studies in mathematics and its applications, volume 15, pages 299-331. Elsevier, 1983. 
[GJLV11] J. B. Garnett, P. W. Jones, T. M. Le, and L. A. Vese. Modeling oscillatory components with the homogeneous spaces $B \dot{M} O^{-\alpha}$ and $\dot{W}^{-\alpha, p}$. Pure Appl. Math. Q., 7(2, Special Issue: In honor of Frederick W. Gehring, Part 2):275-318, 2011.

[GLMV07] J. B. Garnett, T. M. Le, Y. Meyer, and L. A. Vese. Image decompositions using bounded variation and generalized homogeneous Besov spaces. Appl. Comput. Harmon. Anal., 23(1):25-56, 2007.

[GLT89] R. Glowinski and P. Le Tallec. Augmented Lagrangian and operatorsplitting methods in nonlinear mechanics, volume 9 of SIAM Studies in Applied Mathematics. Society for Industrial and Applied Mathematics (SIAM), Philadelphia, PA, 1989.

[GO09] T. Goldstein and S. Osher. The split Bregman method for L1-regularized problems. SIAM J. Imaging Sci., 2(2):323-343, 2009.

[GOSB14] T. Goldstein, B. O’Donoghue, S. Setzer, and R. Baraniuk. Fast alternating direction optimization methods. SIAM J. Imaging Sci., 7(3):1588-1623, 2014.

[Got12] C. Gottschlich. Curved-Region-Based Ridge Frequency Estimation and Curved Gabor Filters for Fingerprint Image Enhancement. IEEE Transactions on Image Processing, 21(4):2220-2227, April 2012.

[GT07] P. Grother and E. Tabassi. Performance of biometric quality measures. IEEE Trans. Pattern Anal. Mach. Intell, 29(4):531-543, 2007.

[Hes69] M. R. Hestenes. Multiplier and gradient methods. J. Optimization Theory Appl., 4:303-320, 1969.

[hle] Classic Test Image Collections. https://www.hlevkin.com/06testimages.htm. Accessed: 2019-09-25.

[HM07] A. Haddad and Y. Meyer. An improvement of Rudin-Osher-Fatemi model. Appl. Comput. Harmon. Anal., 22(3):319-334, 2007.

[HST13] K. He, J. Sun, and X. Tang. Guided image filtering. IEEE transactions on pattern analysis $\mathcal{E}$ machine intelligence, 35(6):1397-1409, 2013.

[HUL93a] J.-B. Hiriart-Urruty and C. Lemaréchal. Convex analysis and minimization algorithms. I, volume 305 of Grundlehren der Mathematischen 
Wissenschaften [Fundamental Principles of Mathematical Sciences]. Springer-Verlag, Berlin, 1993. Fundamentals.

[HUL93b] J.-B. Hiriart-Urruty and C. Lemaréchal. Convex analysis and minimization algorithms. II, volume 306 of Grundlehren der Mathematischen Wissenschaften [Fundamental Principles of Mathematical Sciences]. Springer-Verlag, Berlin, 1993. Advanced theory and bundle methods.

[JKCA03] S. Joun, H. Kim, Y. Chung, and D. Ahn. An Experimental Study on Measuring Image Quality of Infant Fingerprints. In Proc. KES, pages 1261-1269, 2003.

[Jor75] C. Jordan. Essai sur la géométrie à $n$ dimensions. Bull. Soc. Math. France, 3:103-174, 1875.

[JS09] V. Jain and S. Seung. Natural image denoising with convolutional networks. In Advances in Neural Information Processing Systems, pages 769-776, 2009.

[KBPS11] F. Knoll, K. Bredies, T. Pock, and R. Stollberger. Second order total generalized variation (TGV) for MRI. Magnetic resonance in medicine, 65(2):480-491, 2011.

[KEE13] L. Karacan, E. Erdem, and A. Erdem. Structure-preserving Image Smoothing via Region Covariances. ACM Trans. Graph., 32(6):176:1176:11, 2013.

[KOJ05] S. Kindermann, S. Osher, and P. W. Jones. Deblurring and denoising of images by nonlocal functionals. Multiscale Model. Simul., 4(4):10911115, 2005.

[KW87] M. Kass and A. Witkin. Analyzing Oriented Patterns. Computer Vision, Graphics, and Image Processing, 37(3):362-385, 1987.

[LCCK08] S. Lee, H. Choi, K. Choi, and J. Kim. Fingerprint-quality index using gradient components. IEEE Transactions on Information Forensics and Security, 3(4):792-800, 2008.

[Lev05] S. E. Levine. An adaptive variational model for image decomposition. In International Workshop on Energy Minimization Methods in Computer Vision and Pattern Recognition, pages 382-397. Springer, 2005. 
[LHF09] Z. Li, Z. Han, and B. Fu. A novel method for the fingerprint image quality evaluation. In Proc. 2009. CiSE 2009. International Conference on Computational Intelligence and Software Engineering, pages 1-4. IEEE, 2009.

[LJY02] E. Lim, X. Jiang, and W. Yau. Fingerprint Quality and Validity Analysis. In Proc. Int. Conf. on Image Processing, pages 469-472-271, 2002.

[LLV09] T. M. Le, L. H. Lieu, and L. A. Vese. ( $\left.\Phi, \Phi^{*}\right)$ image decomposition models and minimization algorithms. J. Math. Imaging Vision, 33(2):135148, 2009.

[LTS ${ }^{+}$04] E. Lim, K. Toh, P. Suganthan, X. Jiang, and W. Yau. Fingerprint Image Quality Analysis. In Proc. Int. Conf. on Image Processing, pages 12411244, 2004.

[LV05] T. M. Le and L. A. Vese. Image decomposition using total variation and div(BMO). Multiscale Model. Simul., 4(2):390-423, 2005.

[LV08] L. H. Lieu and L. A. Vese. Image restoration and decomposition via bounded total variation and negative Hilbert-Sobolev spaces. Appl. Math. Optim., 58(2):167-193, 2008.

[Mal98] S. Mallat. A wavelet tour of signal processing. Academic Press, Inc., San Diego, CA, 1998.

[Mal02] F. Malgouyres. Minimizing the total variation under a general convex constraint for image restoration. IEEE Trans. Image Process., 11(12):1450-1456, 2002.

[Mey01] Y. Meyer. Oscillating patterns in image processing and nonlinear evolution equations, volume 22 of University Lecture Series. American Mathematical Society, Providence, RI, 2001. The fifteenth Dean Jacqueline B. Lewis memorial lectures.

[MJU17] M. T. McCann, K. H. Jin, and M. Unser. A review of convolutional neural networks for inverse problems in imaging. arXiv preprint arXiv:1710.04011, 2017.

[MMG12] A. Morar, F. Moldoveanu, and E. Gröller. Image segmentation based on active contours without edges. In 2012 IEEE 8th International Conference on Intelligent Computer Communication and Processing, pages 213-220. IEEE, 2012. 
[MMJP09] D. Maltoni, D. Maio, A. K. Jain, and S. Prabhakar. Handbook of fingerprint recognition. Springer Science \& Business Media, 2009.

[MMWJ02] D. Maio, D. Maltoni, J. Wayman, and A. Jain. FVC2000: Fingerprint verification competition. IEEE Transactions on Pattern Analysis and Machine Intelligence, 24(3):402-412, 2002.

[MP07] J. Ma and G. Plonka. Combined curvelet shrinkage and nonlinear anisotropic diffusion. IEEE Trans. Image Process., 16(9):2198-2206, 2007.

[MS89] D. Mumford and J. Shah. Optimal approximations by piecewise smooth functions and associated variational problems. Comm. Pure Appl. Math., 42(5):577-685, 1989.

[NBCE09] K. Ni, X. Bresson, T. Chan, and S. Esedoglu. Local histogram based segmentation using the Wasserstein distance. International journal of computer vision, 84(1):97-111, 2009.

[NFI15] NIST Fingerprint Quality (NFIQ). https://www.nist.gov/servicesresources/software/nist-biometric-image-software-nbis, 2015. Accessed: 2017-12-04.

[NFI16] NFIQ 2.0 NIST fingerprint image quality. https://www.nist.gov/servicesresources/software/development-nfiq-20, 2016. Accessed: 2019-09-25.

[Nik00] M. Nikolova. Local strong homogeneity of a regularized estimator. SIAM J. Appl. Math., 61(2):633-658, 2000.

[Nik02] M. Nikolova. Minimizers of cost-functions involving nonsmooth datafidelity terms. Application to the processing of outliers. SIAM J. Numer. Anal., 40(3):965-994, 2002.

[Nik04] M. Nikolova. A variational approach to remove outliers and impulse noise. J. Math. Imaging Vision, 20(1-2):99-120, 2004. Special issue on mathematics and image analysis.

[NNT10] M. Nikolova, M. K. Ng, and C.-P. Tam. Fast nonconvex nonsmooth minimization methods for image restoration and reconstruction. IEEE Trans. Image Process., 19(12):3073-3088, 2010. 
$\left[\mathrm{OBG}^{+} 05\right]$ S. Osher, M. Burger, D. Goldfarb, J. Xu, and W. Yin. An iterative regularization method for total variation-based image restoration. Multiscale Model. Simul., 4(2):460-489, 2005.

[OOS06] A. Obereder, S. Osher, and O. Scherzer. On the use of the Dual Norms in Bounded Variation Type Regularization, pages 373-390. 2006.

[OR09] E. Outerelo and J. M. Ruiz. Mapping degree theory, volume 108 of Graduate Studies in Mathematics. American Mathematical Society, Providence, RI; Real Sociedad Matemática Española, Madrid, 2009.

[OS04] S. Osher and O. Scherzer. $G$-norm properties of bounded variation regularization. Commun. Math. Sci., 2(2):237-254, 2004.

[OSB16] M. A. Olsen, V. Smida, and C. Busch. Finger image quality assessment features - definitions and evaluation. IET Biometrics, 5:47-64(17), 2016.

[OSV03] S. Osher, A. Solé, and L. Vese. Image decomposition and restoration using total variation minimization and the $H^{-1}$ norm. Multiscale Model. Simul., 1(3):349-370, 2003.

[OXB12] M. A. Olsen, H. Xu, and C. Busch. Gabor filters as candidate quality measure for NFIQ 2.0. In Proc. 2012 5th IAPR International Conference on Biometrics (ICB), pages 158-163. IEEE, 2012.

[PA13] K. Phromsuthirak and V. Areekul. Fingerprint quality assessment using frequency and orientation subbands of block-based Fourier transform. In Proc. 2013 International Conference on Biometrics (ICB), pages 1-7. IEEE, 2013.

[PCP11] N. Pustelnik, C. Chaux, and J.-C. Pesquet. Parallel proximal algorithm for image restoration using hybrid regularization. IEEE Trans. Image Process., 20(9):2450-2462, 2011.

[PM90] P. Perona and J. Malik. Scale-space and edge detection using anisotropic diffusion. IEEE Transactions on Pattern Analysis and Machine Intelligence, 12:629-639, 1990.

[PMD $\left.{ }^{+} 10\right]$ L. Pizarro, P. Mrázek, S. Didas, S. Grewenig, and J. Weickert. Generalised nonlocal image smoothing. Int. J. Comput. Vis., 90(1):62-87, 2010. 
[Pow69] M. J. D. Powell. A method for nonlinear constraints in minimization problems. In Optimization (Sympos., Univ. Keele, Keele, 1968), pages 283-298. Academic Press, London, 1969.

[PS06] G. Plonka and G. Steidl. A multiscale wavelet-inspired scheme for nonlinear diffusion. Int. J. Wavelets Multiresolut. Inf. Process., 4(1):1$21,2006$.

[PS10] V. B. S. Prasath and A. Singh. A hybrid convex variational model for image restoration. Appl. Math. Comput., 215(10):3655-3664, 2010.

[PS14] K. Papafitsoros and C. B. Schönlieb. A combined first and second order variational approach for image reconstruction. J. Math. Imaging Vision, 48(2):308-338, 2014.

[PSWS03] J. Portilla, V. Strela, M. J. Wainwright, and E. P. Simoncelli. Image denoising using scale mixtures of Gaussians in the wavelet domain. IEEE Trans. Image Process., 12(11):1338-1351, 2003.

[Rab92] C. Rabut. Elementary $m$-harmonic cardinal B-splines. Numer. Algorithms, 2(1):39-61, 1992.

[RFP13] H. Raguet, J. Fadili, and G. Peyré. A generalized forward-backward splitting. SIAM J. Imaging Sci., 6(3):1199-1226, 2013.

[RGM+19] R. Richter, C. Gottschlich, L. Mentch, D. H. Thai, and S. F. Huckemann. Smudge Noise for Quality Estimation of Fingerprints and its Validation. IEEE Transactions on Information Forensics and Security, 14(8):19631974, 2019.

[ROF92] L. Rudin, S. Osher, and E. Fatemi. Nonlinear total variation based noise removal algorithms. Physica D., 60(1-4):259-268, 1992.

[SB07] J. Sveinsson and J. Benediktsson. Combined wavelet and curvelet denoising of SAR images using TV segmentation. In Geoscience and Remote Sensing Symposium, 2007. IGARSS 2007. IEEE International, pages 503 - 506, 082007.

[SC03] D. Strong and T. Chan. Edge-preserving and scale-dependent properties of total variation regularization. Inverse Problems, 19(6):S165-S187, 2003. Special section on imaging. 
[SCHP12] C.-T. Shen, F.-J. Chang, Y.-P. Hung, and S.-C. Pei. Edge-preserving image decomposition using L1 fidelity with L0 gradient. In SIGGRAPH Asia 2012 Technical Briefs, page 6. ACM, 2012.

[SGG ${ }^{+}$09] O. Scherzer, M. Grasmair, H. Grossauer, M. Haltmeier, and F. Lenzen. Variational methods in imaging, volume 167 of Applied Mathematical Sciences. Springer, New York, 2009.

[SGPB14] I. W. Selesnick, H. L. Graber, D. S. Pfeil, and R. L. Barbour. Simultaneous low-pass filtering and total variation denoising. IEEE Trans. Signal Process., 62(5):1109-1124, 2014.

[She05] J. Shen. Piecewise $H^{-1}+H^{0}+H^{1}$ images and the Mumford-Shah-Sobolev model for segmented image decomposition. AMRX Appl. Math. Res. Express, (4):143-167, 2005.

[Sim99] E. P. Simoncelli. Bayesian denoising of visual images in the wavelet domain. In Bayesian inference in wavelet-based models, pages 291-308. Springer, 1999.

[SIP] The USC-SIPI Image Database. http://sipi.usc.edu/database/. Accessed: 2019-09-25.

[SKK01] L. Shen, A. Kot, and W. Koo. Quality Measures of Fingerprint Images. In International Conference on Audio-and-Video-Based Biometric Person Authentication, pages 266-271. Springer, 2001.

[SS03] E. M. Stein and R. Shakarchi. Fourier analysis, volume 1 of Princeton Lectures in Analysis. Princeton University Press, Princeton, NJ, 2003. An introduction.

[SS08] S. Setzer and G. Steidl. Variational methods with higher-order derivatives in image processing. In Approximation theory XII: San Antonio 2007, Mod. Methods Math., pages 360-385. Nashboro Press, Brentwood, TN, 2008.

[SS12] D. Szolgay and T. Szirányi. Adaptive Image Decomposition Into Cartoon and Texture Parts Optimized by the Orthogonality Criterion. IEEE transactions on image processing : a publication of the IEEE Signal Processing Society, 21:3405-3415, 042012. 
[SSD09] K. Subr, C. Soler, and F. Durand. Edge-preserving multiscale image decomposition based on local extrema. ACM Transactions on Graphics (TOG), 28(5):147, 2009.

[SWB ${ }^{+}$04] G. Steidl, J. Weickert, T. Brox, P. Mrázek, and M. Welk. On the equivalence of soft wavelet shrinkage, total variation diffusion, total variation regularization, and sides. SIAM J. Numer. Anal., 42(2):686-713, 2004.

[TG16] D. H. Thai and C. Gottschlich. Global Variational Method for Fingerprint Segmentation by Three-part Decomposition. IET Biometrics, 5(2):120 $130,2016$.

[TH13] L. Tang and C. He. Multiscale Texture Extraction with Hierarchical $\left(\mathrm{BV}, G_{p}, L^{2}\right)$ Decomposition. Journal of Mathematical Imaging and Vision, 45(2):148-163, 2013.

[THG16] D. Thai, S. Huckemann, and C. Gottschlich. Filter Design and Performance Evaluation for Fingerprint Image Segmentation. PloS ONE, 11(5):e0154160, 2016.

[TL17] R. F. Teixeira and N. J. Leite. A new framework for quality assessment of high-resolution fingerprint images. IEEE transactions on pattern analysis and machine intelligence, 39(10):1905-1917, 2017.

[TM98] C. Tomasi and R. Manduchi. Bilateral filtering for gray and color images. In Computer Vision, 1998. Sixth International Conference on, pages 839-846. IEEE, 1998.

[TYZ $\left.{ }^{+} 12\right]$ X. Tao, X. Yang, Y. Zang, X. Jia, and J. Tian. A novel measure of fingerprint image quality using principal component analysis (PCA). In Proc. 2012 5th IAPR International Conference on Biometrics (ICB), pages 170-175. IEEE, 2012.

[TZM12] C. Tada, A. Zaghetto, and B. Macchiavello. Fingerprint image quality estimation using a fuzzy inference system. In Proc. Seventh International Conference on Forensic Computer Science (ICoFCS 2012), volume 10, page C2012007, 2012.

[USVDV09] M. Unser, D. Sage, and D. Van De Ville. Multiresolution monogenic signal analysis using the Riesz-Laplace wavelet transform. IEEE Trans. Image Process., 18(11):2402-2418, 2009. 
[UVDV10] M. Unser and D. Van De Ville. Wavelet steerability and the higher-order Riesz transform. IEEE Trans. Image Process., 19(3):636-652, 2010.

[VDVBU05] D. Van De Ville, T. Blu, and M. Unser. Isotropic polyharmonic Bsplines: scaling functions and wavelets. IEEE Trans. Image Process., 14(11):1798-1813, 2005.

[VO03] L. A. Vese and S. J. Osher. Modeling textures with total variation minimization and oscillating patterns in image processing. J. Sci. Comput., 19(1-3):553-572, 2003. Special issue in honor of the sixtieth birthday of Stanley Osher.

[VO04] L. A. Vese and S. J. Osher. Image denoising and decomposition with total variation minimization and oscillatory functions. J. Math. Imaging Vision, 20(1-2):7-18, 2004. Special issue on mathematics and image analysis.

[Wei98] J. Weickert. Anisotropic diffusion in image processing. European Consortium for Mathematics in Industry. B. G. Teubner, Stuttgart, 1998.

[WOG06] H. Winnemöller, S. C. Olsen, and B. Gooch. Real-time video abstraction. ACM Transactions On Graphics (TOG), 25(3):1221-1226, 2006.

[WT10] C. Wu and X.-C. Tai. Augmented Lagrangian method, dual methods, and split Bregman iteration for ROF, vectorial TV, and high order models. SIAM J. Imaging Sci., 3(3):300-339, 2010.

[WWH06] G. Wang, T.-T. Wong, and P.-A. Heng. Deringing cartoons by image analogies. ACM Transactions on Graphics (TOG), 25(4):1360-1379, 2006.

[WXSL08] J. Wu, S. Xie, D.-H. Seo, and W. Lee. A new approach for classification of fingerprint image quality. In Proc. 2008. ICCI 2008. 7th IEEE International Conference on Cognitive Informatics, pages 375-383. IEEE, 2008.

[WYYZ08] Y. Wang, J. Yang, W. Yin, and Y. Zhang. A new alternating minimization algorithm for total variation image reconstruction. SIAM J. Imaging Sci., 1(3):248-272, 2008.

[XLXJ11] L. Xu, C. Lu, Y. Xu, and J. Jia. Image Smoothing via L0 Gradient Minimization. ACM Trans. Graph., 30(6):174:1-174:12, December 2011. 
[XXC12] J. Xie, L. Xu, and E. Chen. Image denoising and inpainting with deep neural networks. In Advances in neural information processing systems, pages 341-349, 2012.

[XYG $\left.{ }^{+} 12\right]$ S. Xie, J. Yang, H. Gong, S. Yoon, and D. Park. Intelligent fingerprint quality analysis using online sequential extreme learning machine. Soft Computing-A Fusion of Foundations, Methodologies and Applications, 16(9):1555-1568, 2012.

[XYXJ12] L. Xu, Q. Yan, Y. Xia, and J. Jia. Structure Extraction from Texture via Natural Variation Measure. ACM Transactions on Graphics (SIGGRAPH Asia), 31(6):1-10, 2012.

[YCLJ13] S. Yoon, K. Cao, E. Liu, and A. Jain. LFIQ: Latent fingerprint image quality. In IEEE Sixth International Conference on Biometrics: Theory, Applications and Systems (BTAS), pages 1-8, Arlington, VA, USA, September 2013.

[YGO05] W. Yin, D. Goldfarb, and S. Osher. Image cartoon-texture decomposition and feature selection using the total variation regularized $\mathrm{L} 1$ functional. In Variational, Geometric, and Level Set Methods in Computer Vision, pages 73-84. Springer, 2005.

[YGO07] W. Yin, D. Goldfarb, and S. Osher. A comparison of three total variation based texture extraction models. Journal of Visual Communication and Image Representation, 18(3):240-252, 2007.

[YLBCR15] Z. Yao, J.-M. Le Bars, C. Charrier, and C. Rosenberger. Fingerprint quality assessment combining blind image quality, texture and minutiae features. In Proc. 2015 International Conference on Information Systems Security and Privacy (ICISSP), pages 315-321, 2015.

[YLCR16] Z. Yao, J.-M. Le Bars, C. Charrier, and C. Rosenberger. Literature review of fingerprint quality assessment and its evaluation. IET Biometrics, 5(3):243-251, September 2016.

[YSLX16] Y. Yang, J. Sun, H. Li, and Z. Xu. Deep ADMM-Net for compressive sensing MRI. In 30th Conference on Neutral Information Processing Systems (NIPS 2016), pages 10-18, 2016.

[ZK13] P. Zhu and A. V. Knyazev. Angles between subspaces and their tangents. J. Numer. Math., 21(4):325-340, 2013. 
[ZZGZ17] K. Zhang, W. Zuo, S. Gu, and L. Zhang. Learning deep CNN denoiser prior for image restoration. In IEEE Conference on Computer Vision and Pattern Recognition, volume 2, pages 3929-3938, 2017. 